\title{
The Catalytic Asymmetric Fischer Indolization
}

Steffen Müller, Matthew J. Webber, and Benjamin List*

Max-Planck-Institut für Kohlenforschung, Kaiser-Wilhelm-Platz 1, D-45470, Mülheim an der Ruhr, Germany

list@mpi-muelheim.mpg.de

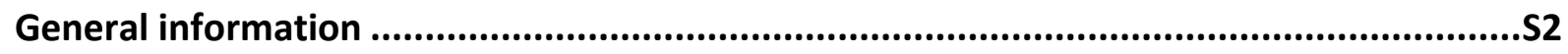

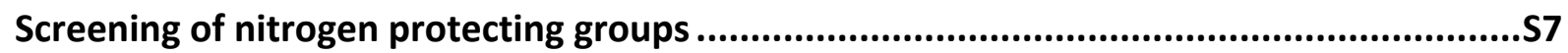

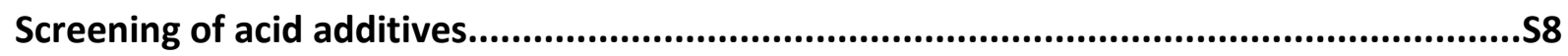

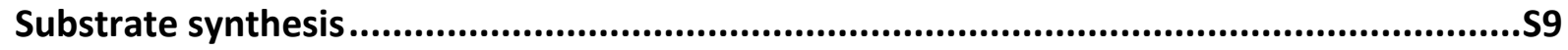

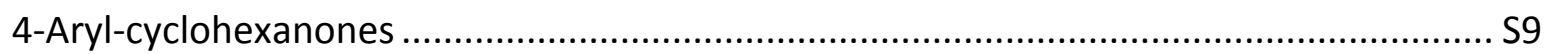

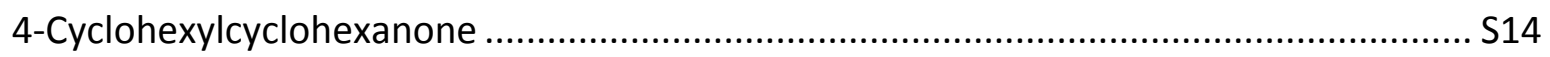

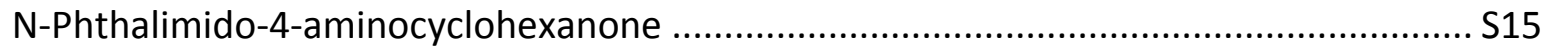

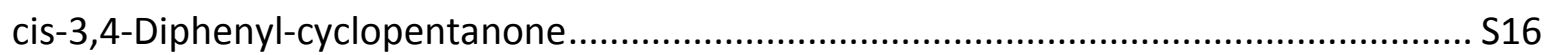

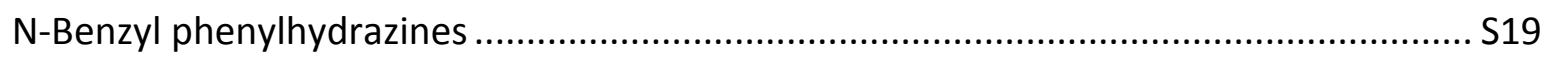

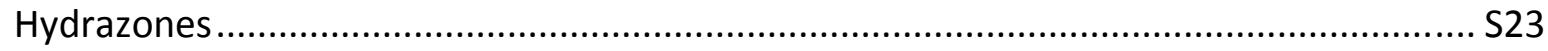

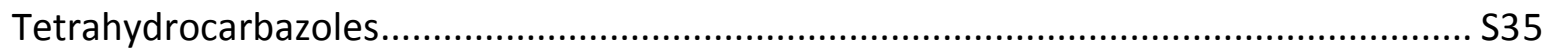

Large scale indolization and formal synthesis of $(S)$-Ramatroban ..................................S50

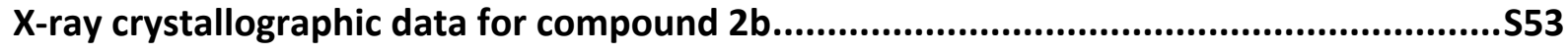

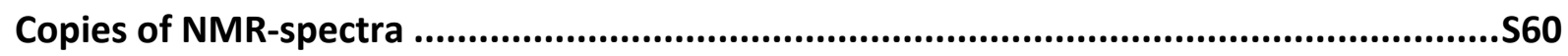

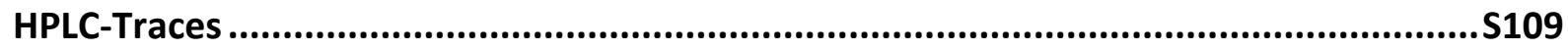




\section{General information}

Unless otherwise stated, all reagents were purchased from commercial suppliers and used without further purification. All solvents used in the reactions were distilled from appropriate drying agents prior to use. Reactions were monitored by thin layer chromatography (TLC) on silica gel pre-coated plastic sheets $(0.2 \mathrm{~mm}$, Machery-Nagel). Visualization was accomplished by irradiation with UV light at $254 \mathrm{~nm}$ and/or phosphomolybdic acid (PMA) or anisaldehyde stain. Column chromatography was performed on Merck silica gel (60, particle size 0.040-0.063 mm). Melting points (Mp) were measured using a Büchi Melting Point B-540 apparatus and are uncorrected. Optical rotations were measured on a Autopol IV automatic polarimter (Rudolph Research Analytical). Proton, and carbon NMR spectra were recorded on Bruker AV-500, Bruker AV400 or DPX-300 spectrometer in deuterated solvents. Proton chemical shifts are reported in $\mathrm{ppm}(\delta)$ relative to tetramethylsilane (TMS) with the solvent resonance employed as the internal standard $\left(\mathrm{CD}_{2} \mathrm{Cl}_{2}, \delta 5.32 \mathrm{ppm} ; \mathrm{CDCl}_{3} \delta 7.24 \mathrm{ppm}\right)$. Data are reported as follows: chemical shift, multiplicity $(s=$ singlet, $d=$ doublet, $q=$ quartet, sept = septet, $m=$ multiplet, $\mathrm{br}=$ broad), coupling constants $(\mathrm{Hz})$ and integration. ${ }^{13} \mathrm{C}$ chemical shifts are reported in $\mathrm{ppm}$ ( $\delta)$ from tetramethylsilane (TMS) with the solvent resonance as the internal standard $\left(\mathrm{CD}_{2} \mathrm{Cl}_{2}, \delta 53.8 \mathrm{ppm} ; \mathrm{CDCl}_{3}, \delta 77.16 \mathrm{ppm}\right)$. High resolution mass spectra were determined on a Bruker APEX III FTMS (7 T magnet). The enantiomeric ratios were determined by HPLC analysis employing a chiral stationary phase column specified in the individual experiment, by comparing the samples with the appropriate racemic mixtures. 
Synthesis of catalyst $5 f$

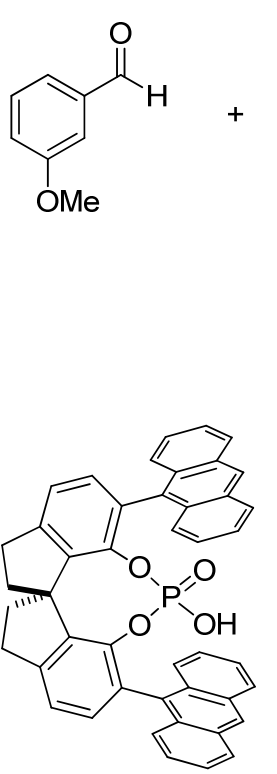

$(R)-5 \mathbf{f}$<smiles>CCCCCCCC(C)=O</smiles><smiles>Oc1cccc2c1C1(CC2)CCc2cccc(O)c21</smiles>

rac-A $\stackrel{[\text { Ref. 2] }}{\longrightarrow}$<smiles>Oc1cccc2c1C1CC(CC2)c2cccc(O)c21</smiles>

(S)-A<smiles>Oc1cccc2c1C1c3c(O)cccc3CC1C2</smiles>

$(R)-\mathbf{A}$

[Ref. 3]

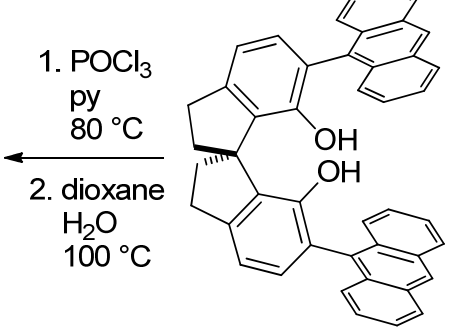

$(R)-\mathrm{D}$

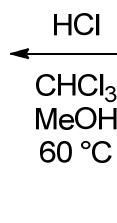

$\mathrm{MeOH}$ $60^{\circ} \mathrm{C}$

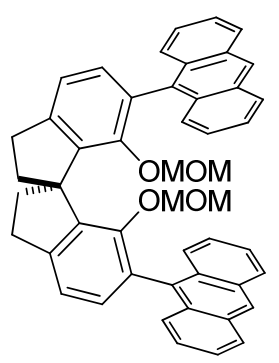

(R)-C

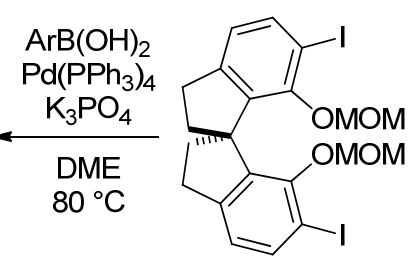

(R)-B

Racemic SPINOL (rac-A) was synthesized starting from acetone and 3-methoxybenzaldehyde according to the procedure reported by Birman et al. with slight modifications. ${ }^{1}$

The resolution of racemic SPINOL was accomplished following a literature reported method. ${ }^{2}$ For the MOM-protection and diiodination of enantiomercially pure $(R)$-SPINOL $((R)-\mathrm{A})$ the protocol reported by Zhou and co-workers was followed with minor modifications. ${ }^{3}$

(1) Birman, V. B.; Rheingold, A. L.; Lam, K.-C. Tetrahedron: Asymmetry 1990, 10, 125.

(2) Zhang, J.-H.; Liao, J.; Cui, X.; Yu, K.-B.; Zhu, J.; Deng, J.-G.; Zhu, S.-F.; Wang, L.-X.; Zhou, Q.-L.; Chung, L. W.;

Ye, T. Tetrahedron: Asymmetry 2002, 13, 1363.

(3) Yang, Y.; Zhu, S.-F.; Duan, H.-F.; Zhou, C.-Y.; Wang, L.-X.; Zhou, Q.-L. J. Am. Chem. Soc. 2007, 129, 2248. 
$(R)-7,7^{\prime}-$ Bis(methoxymethoxy)-6,6'-bis(9-anthracenyl)-1,1'-spirobiindane ((R)-C)

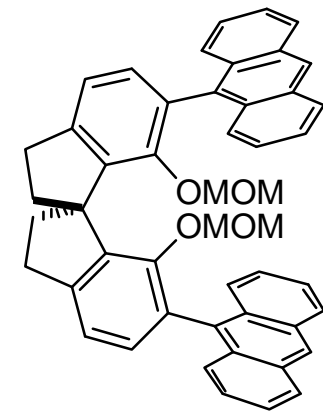

$(R)-\mathrm{C}$

A Schlenk tube was charged with (R)-6,6'-Diiodo-7,7'bis(methoxymethoxy)-2,2',3,3'-tetrahydro-1,1'-spirobiindane $\quad((R)$-B) (200 mg, $0.338 \mathrm{mmol}$ ), 9-anthracene boronic acid (300 mg, $1.35 \mathrm{mmol}$ ) and $\mathrm{K}_{3} \mathrm{PO}_{4}(573 \mathrm{mg}, 2.70 \mathrm{mmol})$. The solids were set under argon, taken up in DME (3 mL) and the resulting suspension was degassed. After 15 $\min \mathrm{Pd}\left(\mathrm{PPh}_{3}\right)_{4}(39.0 \mathrm{mg}, 0.034 \mathrm{mmol})$ was added and degassing was continued for further $5 \mathrm{~min}$ before the tube was sealed and heated to $80{ }^{\circ} \mathrm{C}$ for $48 \mathrm{~h}$. After cooling to room temperature $\mathrm{H}_{2} \mathrm{O}$ and $\mathrm{CH}_{2} \mathrm{Cl}_{2}$ were added and the layers were separated. The aqueous layer was extracted with $\mathrm{CH}_{2} \mathrm{Cl}_{2}$, the combined extracts were dried over $\mathrm{MgSO}_{4}$ and evaporated to dryness. Purification of the residue by repeated column chromatography on $\mathrm{SiO}_{2}$ (hexane: $\mathrm{CH}_{2} \mathrm{Cl}_{2} 2: 1$ ) yielded the title compound $89.3 \mathrm{mg}(0.129 \mathrm{mmol}, 38 \%)$ as a colorless solid.

${ }^{1} \mathrm{H}-\mathrm{NMR}\left(400 \mathrm{MHz}, \mathrm{CD}_{2} \mathrm{Cl}_{2}\right) \delta=8.52(\mathrm{~s}, 2 \mathrm{H}), 8.07(\mathrm{~d}, J=8.1 \mathrm{~Hz}, 2 \mathrm{H}), 8.00(\mathrm{~d}, J=8.5 \mathrm{~Hz}, 2 \mathrm{H})$, $7.95(\mathrm{~d}, J=8.9 \mathrm{~Hz}, 2 \mathrm{H}), 7.60(\mathrm{dd}, J=8.8,0.9 \mathrm{~Hz}, 2 \mathrm{H}), 7.51-7.42(\mathrm{~m}, 4 \mathrm{H}), 7.23-7.19(\mathrm{~m}, 2 \mathrm{H})$, $7.15(\mathrm{~d}, J=7.5 \mathrm{~Hz}, 2 \mathrm{H}), 7.05(\mathrm{~d}, J=7.5 \mathrm{~Hz}, 2 \mathrm{H}), 6.30-6.26(\mathrm{~m}, 2 \mathrm{H}), 4.32(\mathrm{~d}, J=5.7 \mathrm{~Hz}, 2 \mathrm{H}), 3.84$ (d, $J=5.7 \mathrm{~Hz}, 2 \mathrm{H}), 3.33-3.25(\mathrm{~m}, 2 \mathrm{H}), 3.14$ (ddd, $J=15.8,8.5,1.2 \mathrm{~Hz}, 2 \mathrm{H}), 2.69-2.61(\mathrm{~m}, 2 \mathrm{H})$, 2.56 (ddd, $J=12.4,7.9,1.8 \mathrm{~Hz}, 2 \mathrm{H}), 2.47(\mathrm{~s}, 6 \mathrm{H})$.

${ }^{13} \mathrm{C}-\mathrm{NMR}\left(100 \mathrm{MHz}, \mathrm{CD}_{2} \mathrm{Cl}_{2}\right) \delta=154.1,146.0,142.2,134.8,132.7,131.9,131.6,130.9,130.5$, $128.9,128.8,127.9,127.8,127.2,126.9,126.3,125.9,125.8,125.3,120.2,98.6,60.5,56.4$, 39.4, $31.6 \mathrm{ppm}$.

MS (EI) m/z (\%): 692 (100), 660 (29), 648 (12), 628 (32), 616 (86), 346 (11), 295 (27), 178 (13), 45 (8).

HRMS (ESI+) $m / z$ calculated for $\mathrm{C}_{49} \mathrm{H}_{40} \mathrm{O}_{4} \mathrm{Na}\left(\mathrm{M}+\mathrm{Na}^{+}\right) 715.281878$, found 715.282206 . 
$(R)-6,6^{\prime}$-Bis(9-anthracenyl)-1,1'-spirobiindane-7,7'-diol ((R)-D)

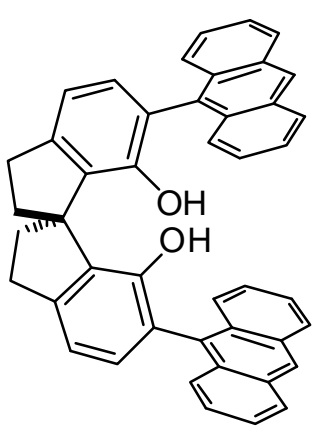

$(R)-\mathrm{D}$

A solution of $(R)-7,7^{\prime}$-bis(methoxymethoxy)-6,6'-bis(9-anthracenyl)-1,1'spirobiindane $((R)-\mathrm{C})(89.3 \mathrm{mg}, 0.129 \mathrm{mmol})$ in a mixture of $\mathrm{CHCl}_{3}(5 \mathrm{~mL})$ and $\mathrm{MeOH}(5 \mathrm{~mL})$ was treated with conc. $\mathrm{HCl}(1 \mathrm{~mL})$ and heated to $60^{\circ} \mathrm{C}$ for $4 \mathrm{~h}$. After cooling to room temperature saturated $\mathrm{NaHCO}_{3}$ solution was added, the layers were separated and the aqueous layer was extracted with $\mathrm{CH}_{2} \mathrm{Cl}_{2}$. The combined organic layers were dried over $\mathrm{MgSO}_{4}$ and evaporated to dryness. Purification of the residue on $\mathrm{SiO}_{2}$ (hexane: $\mathrm{CH}_{2} \mathrm{Cl}_{2} 2: 1$ ) gave the product $(R)-\mathbf{D} 77.1 \mathrm{mg}(1.27 \mathrm{mmol}, 98 \%)$ as a yellow solid.

${ }^{1} \mathrm{H}-\mathrm{NMR}\left(500 \mathrm{MHz}, \mathrm{CDCl}_{3}\right) \delta=8.51(\mathrm{~s}, 2 \mathrm{H}), 8.05(\mathrm{~d}, J=8.4 \mathrm{~Hz}, 2 \mathrm{H}), 8.00(\mathrm{~d}, J=8.4,2 \mathrm{H}), 7.81$ (d, $J=8.7 \mathrm{~Hz}, 2 \mathrm{H}), 7.48(\mathrm{dd}, J=8.1,6.7 \mathrm{~Hz}, 2 \mathrm{H}), 7.41(\mathrm{dd}, J=8.0,7.1 \mathrm{~Hz}, 2 \mathrm{H}), 7.34(\mathrm{~d}, J=8.8$ $\mathrm{Hz}, 2 \mathrm{H}$ ), 7.27 (dd, J= 7.3, $6.8 \mathrm{~Hz}, 2 \mathrm{H}$ ), 7.08 (d, J=7.5 Hz, 2H), 7.00 (d, J=7.5 Hz, 2H), 6.48 (dd, $J=8.1,7.2 \mathrm{~Hz}, 2 \mathrm{H}), 4.59(\mathrm{~s}, 2 \mathrm{H}), 3.30-3.23(\mathrm{~m}, 2 \mathrm{H}), 3.15(\mathrm{dd}, J=14.7,8.8 \mathrm{~Hz}, 2 \mathrm{H}), 2.64-2.53$ $(\mathrm{m}, 4 \mathrm{H}) \mathrm{ppm}$.

${ }^{13} \mathrm{C}-\mathrm{NMR}\left(125 \mathrm{MHz}, \mathrm{CDCl}_{3}\right) \delta=150.8,145.8,133.5,131.7,131.7,131.5,131.1,131.0,130.8$, $128.7,128.2,127.4,126.6,126.3,126.3,125.9,125.5,125.4,122.8,117.0,59.0,38.4,31.8$ ppm.

MS (EI) m/z (\%): 604 (100), 575 (5), 321 (5), 302 (30), 283 (8).

HRMS $(E S I+) m / z$ calculated for $\mathrm{C}_{45} \mathrm{H}_{32} \mathrm{O}_{2} \mathrm{Na}\left(\mathrm{M}+\mathrm{Na}^{+}\right) 627.229447$, found 627.229264. 
$(R)-6,6$ '-Bis(9-anthracenyl)-1,1'-spirobiindane-7,7'-diyl-hydrogenphosphate $((R)-5 \mathbf{f})$

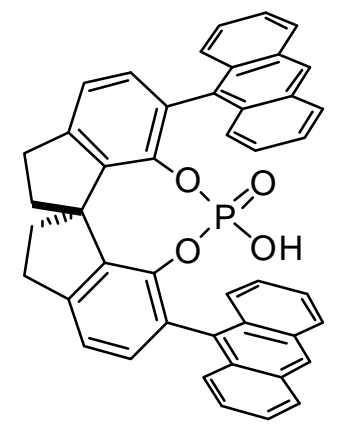

$(R)-5 f$

At $0{ }^{\circ} \mathrm{CPOCl}_{3}(98.5 \mathrm{mg}, 59 \mu \mathrm{L}, 0.642 \mathrm{mmol})$ was added to a solution of diol $(R)-D(77.7 \mathrm{mg}, 0.128 \mathrm{mmol})$ in anhydrous pyridine $(1.5 \mathrm{~mL})$ and the resulting mixture was heated to $80^{\circ} \mathrm{C}$ in a stopper sealed flask. After 24 $\mathrm{h}$ a white precipitate was formed and the mixture was again cooled to 0 ${ }^{\circ} \mathrm{C}$. 1,4-Dioxane $(2 \mathrm{~mL})$ and $\mathrm{H}_{2} \mathrm{O}(0.6 \mathrm{~mL})$ were added and the reaction was heated to $100{ }^{\circ} \mathrm{C}$ for $48 \mathrm{~h}$ until the precipitate had completely dissolved. At ambient temperature the mixture was acidified with $10 \%$

$\mathrm{HCl}$ and extracted with $\mathrm{CH}_{2} \mathrm{Cl}_{2}$. The combined organic layers were dried over $\mathrm{MgSO}_{4}$, the solvent was removed under reduced pressure and the residue purified by column chromatography on $\mathrm{SiO}_{2}$ using $\mathrm{CH}_{2} \mathrm{Cl}_{2}: \mathrm{MeOH}(50: 1$ to $20: 1)$ as the eluent. The obtained product was redissolved in $\mathrm{CH}_{2} \mathrm{Cl}_{2}(50 \mathrm{~mL})$ and thoroughly washed with $4 \mathrm{M} \mathrm{HCl}$ to remove salt impurities and completely protonate the catalyst. The organic layer was separated and evaporated to dryness. The residue was dried in vacuo ( $10^{-2} \mathrm{mbar}$ ) for $24 \mathrm{~h}$ to give the catalyst $(R)-5 f 69.3 \mathrm{mg}(0.104 \mathrm{mmol}, 81 \%)$ as a light yellow solid.

Note: Sometimes the catalyst is obtained as a 2:1 complex with 1,4-dioxane, which is resistant towards further drying. However, in the reaction we did not observe any differences in the performance of catalyst $\mathbf{5} f$ and the 2:1 complex with 1,4-dioxane.

${ }^{1} \mathrm{H}-\mathrm{NMR}\left(500 \mathrm{MHz}, \mathrm{CD}_{2} \mathrm{Cl}_{2}\right) \delta=8.25(\mathrm{~s}, 2 \mathrm{H}), 7.92(\mathrm{~d}, J=7.6 \mathrm{~Hz}, 2 \mathrm{H}), 7.74(\mathrm{~d}, J=8.9 \mathrm{~Hz}, 2 \mathrm{H})$, $7.65(\mathrm{~d}, J=8.2 \mathrm{~Hz}, 4 \mathrm{H}), 7.42-7.37(\mathrm{~m}, 6 \mathrm{H}), 7.25(\mathrm{~d}, J=7.6 \mathrm{~Hz}, 2 \mathrm{H}), 7.22$ (dd, $J=7.8,7.0 \mathrm{~Hz}$, $2 \mathrm{H}), 7.13(\mathrm{dd}, J=8.4,7.1 \mathrm{~Hz}, 2 \mathrm{H}), 3.98(\mathrm{br}, 1 \mathrm{H}), 3.40-3.33(\mathrm{~m}, 2 \mathrm{H}), 3.13(\mathrm{dd}, J=16.6,7.9 \mathrm{~Hz}$, $2 \mathrm{H}), 2.59(\mathrm{dd}, J=12.1,6.5 \mathrm{~Hz}, 2 \mathrm{H}), 2.49-2.43(\mathrm{~m}, 2 \mathrm{H}) \mathrm{ppm}$.

${ }^{13} \mathrm{C}-\mathrm{NMR}\left(125 \mathrm{MHz}, \mathrm{CD}_{2} \mathrm{Cl}_{2}\right) \delta=146.8,144.3(\mathrm{~d}, J=8.3 \mathrm{~Hz}), 140.7,133.5,132.4,131.8,131.2$, 131.0, 130.4 (d, $J=3.9 \mathrm{~Hz}$ ), 130.0, 128.8, 128.4, 128.1, 127.4, 126.5, 126.1, 125.2, 125.2, $124.9,122.8,60.5,39.3,30.7 \mathrm{ppm}$

${ }^{31}$ P-NMR $\left(202 \mathrm{MHz}, \mathrm{CD}_{2} \mathrm{Cl}_{2}\right) \delta=-12.7 \mathrm{ppm}$.

MS (EI) m/z (\%): 666 (100), 333 (25), 265 (4).

HRMS (ESI-) $m / z$ calculated for $\mathrm{C}_{45} \mathrm{H}_{30} \mathrm{O}_{4} \mathrm{P}\left(\mathrm{M}-\mathrm{H}^{+}\right)$665.188727, found 665.188513 .

$[\alpha]_{D}^{25}: 445^{\circ}, \mathrm{c}=0.28\left(\mathrm{CH}_{2} \mathrm{Cl}_{2}\right)$. 


\section{Screening of nitrogen protecting groups}

To investigate the influence of different protecting groups on the enantioselectivity of the reaction, a variety of differently protected hydrazones were allowed to react in a sealed vial in the presence of catalysts $\mathbf{3 a}(10 \mathrm{~mol} \%)$ and $\mathbf{3 f}(10 \mathrm{~mol} \%)$. The reactions were conducted in benzene at $80^{\circ} \mathrm{C}$ and stopped after a significant amount of product had formed (determined by TLC). A sample of the reaction mixture was submitted to preparative TLC and the obtained product was analyzed by HPLC analysis on a chiral stationary phase.
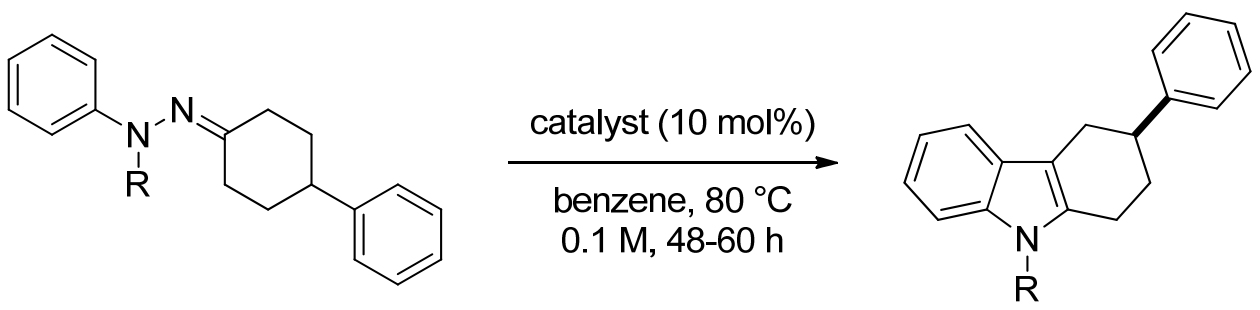

\begin{tabular}{|c|c|c|c|}
\hline entry & $\mathrm{R}$ & $\begin{array}{c}\text { catalyst 3a } \\
\text { er }\end{array}$ & $\begin{array}{c}\text { catalyst } \mathbf{3 f} \\
\text { er }\end{array}$ \\
\hline 1 & $\mathrm{Bn}$ & $51: 49$ & $78.5: 21.5$ \\
\hline 2 & $\mathrm{CBz}$ & $46.5: 53.5$ & $52.5: 47.5$ \\
\hline 3 & $\mathrm{Ph}_{2}-\mathrm{CH}$ & $42: 58$ & $39.5: 60.5$ \\
\hline 4 & $\beta$-naphthyl- $\mathrm{CH}_{2}$ & $44: 56$ & $17.5: 82.5$ \\
\hline 5 & 2-Me- $\mathrm{C}_{6} \mathrm{H}_{4}-\mathrm{CH}_{2}$ & $56: 44$ & $20.5: 79.5$ \\
\hline 6 & $3,5-\mathrm{Me}_{2}-\mathrm{C}_{6} \mathrm{H}_{3}-\mathrm{CH}_{2}$ & $51.5: 38.5$ & 79:21 \\
\hline 7 & $3,5-(\mathrm{MeO})_{2}-\mathrm{C}_{6} \mathrm{H}_{3}-\mathrm{CH}_{2}$ & $57.5: 42.5$ & $79.5: 20.5$ \\
\hline 8 & $3,5-\left(\mathrm{CF}_{3}\right)_{2}-\mathrm{C}_{6} \mathrm{H}_{3}-\mathrm{CH}_{2}$ & $38: 62$ & $22.5: 77.5$ \\
\hline 9 & $3,5-\mathrm{F}_{2}-\mathrm{C}_{6} \mathrm{H}_{3}-\mathrm{CH}_{2}$ & $40.5: 59.5$ & $21: 79$ \\
\hline 10 & 4-Me- $\mathrm{C}_{6} \mathrm{H}_{4}-\mathrm{CH}_{2}$ & $52: 48$ & $80: 20$ \\
\hline 11 & $4-\mathrm{F}-\mathrm{C}_{6} \mathrm{H}_{4}-\mathrm{CH}_{2}$ & $48.5: 51.5$ & $81: 19$ \\
\hline 12 & $4-\mathrm{Br}-\mathrm{C}_{6} \mathrm{H}_{4}-\mathrm{CH}_{2}$ & $50: 50$ & $82.5: 17.5$ \\
\hline 13 & $4-\mathrm{I}-\mathrm{C}_{6} \mathrm{H}_{4}-\mathrm{CH}_{2}$ & $51.5: 48.5$ & $83.5: 16.5$ \\
\hline 14 & 4-Ph- $\mathrm{C}_{6} \mathrm{H}_{4}-\mathrm{CH}_{2}$ & $44: 56$ & $16.5: 83.5$ \\
\hline 15 & 4-tBu- $\mathrm{C}_{6} \mathrm{H}_{4}-\mathrm{CH}_{2}$ & $41.5: 58.5$ & $18.5: 81.5$ \\
\hline
\end{tabular}




\section{Screening of acid additives}

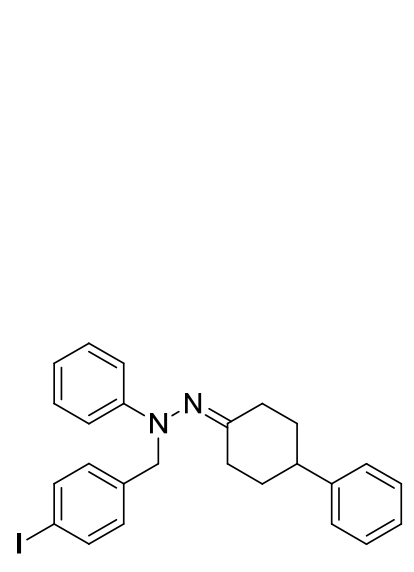

1b
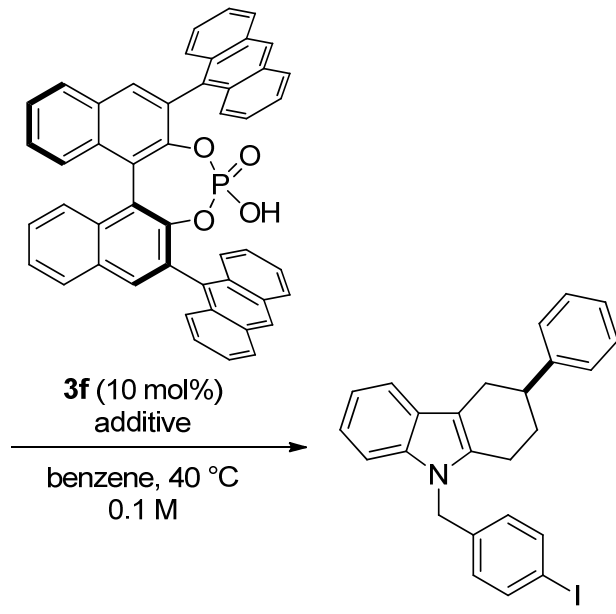

2b

The reactions were run on $0.025 \mathrm{mmol}$ scale with catalyst $3 \mathrm{f}(10 \mathrm{~mol} \%)$ in benzene $(0.25 \mathrm{~mL})$ at $40{ }^{\circ} \mathrm{C}$ with different additives. The conversion was monitored by TLC. After the indicated time a sample of the reaction mixture was purified by preparative thin layer chromatography and the enatiomeric ratio of the product $\mathbf{2} \mathbf{b}$ was determined by HPLC analysis on a chiral stationary phase.

\begin{tabular}{|c|c|c|c|c|c|}
\hline \multirow[b]{2}{*}{ entry } & \multirow[b]{2}{*}{ additive } & \multirow[b]{2}{*}{$\mathrm{t}$} & \multirow[b]{2}{*}{ er } & \multicolumn{2}{|c|}{ conversion (by TLC) } \\
\hline & & & & complete & incomplete \\
\hline 1 & - & $7 d$ & $89.5: 10.5$ & & $x$ \\
\hline 2 & $\mathrm{AcOH}$ (1 eq.) & $7 d$ & $76: 24$ & & $x$ \\
\hline 3 & t-BuCO ${ }_{2} \mathrm{H}$ (1 eq.) & $7 d$ & $82.5: 17.5$ & & $x$ \\
\hline 4 & $\mathrm{C}_{6} \mathrm{H}_{5} \mathrm{CO}_{2} \mathrm{H}$ (1 eq.) & $7 d$ & $74.5: 25.5$ & & $x$ \\
\hline 5 & 4- $\mathrm{NO}_{2}-\mathrm{C}_{6} \mathrm{H}_{4} \mathrm{CO}_{2} \mathrm{H}$ (1 eq.) & $7 d$ & $74: 26$ & & $x$ \\
\hline 6 & 4-MeO- $\mathrm{C}_{6} \mathrm{H}_{4} \mathrm{CO}_{2} \mathrm{H}$ (1 eq.) & $7 d$ & $81: 19$ & & $x$ \\
\hline 7 & 2- $\mathrm{MeO}-\mathrm{C}_{6} \mathrm{H}_{4} \mathrm{CO}_{2} \mathrm{H}$ (1 eq.) & $7 d$ & $82.5: 17.5$ & & $\mathrm{x}$ \\
\hline 8 & $\begin{array}{l}\text { Amberlyst } 15(10 \mathrm{mg}) \\
\left(\approx 1.88 \text { eq. } \mathrm{R}-\mathrm{SO}_{3} \mathrm{H}\right)\end{array}$ & $5 d$ & $82: 18$ & $x$ & \\
\hline 9 & $\begin{array}{l}\text { Amberlyst } 15(20 \mathrm{mg}) \\
\left(\approx 3.76 \text { eq. } \mathrm{R}-\mathrm{SO}_{3} \mathrm{H}\right)\end{array}$ & $5 d$ & $77.5: 22.5$ & $x$ & \\
\hline 10 & $\begin{array}{c}\text { Amberlite CG } 50(10 \mathrm{mg}) \\
\left(\approx 4 \text { eq. } \mathrm{R}-\mathrm{CO}_{2} \mathrm{H}\right)\end{array}$ & $5 d$ & $89.5: 10.5$ & & $x$ \\
\hline 11 & $\begin{array}{c}\text { Amberlite CG } 50(20 \mathrm{mg}) \\
\left(\approx 8 \text { eq. } \mathrm{R}-\mathrm{CO}_{2} \mathrm{H}\right)\end{array}$ & $5 d$ & $89: 11$ & & $x$ \\
\hline 12 & $\begin{array}{l}\text { Amberlite CG } 50(50 \mathrm{mg}) \\
\quad\left(\approx 20 \text { eq. } \mathrm{R}-\mathrm{CO}_{2} \mathrm{H}\right)\end{array}$ & $5 d$ & $88: 12$ & $x$ & \\
\hline
\end{tabular}




\section{Substrate synthesis}

\section{4-Aryl-cyclohexanones}

All 4-aryl cyclohexanones were prepared from 1,4-dioxaspiro[4.5]decan-8-one.

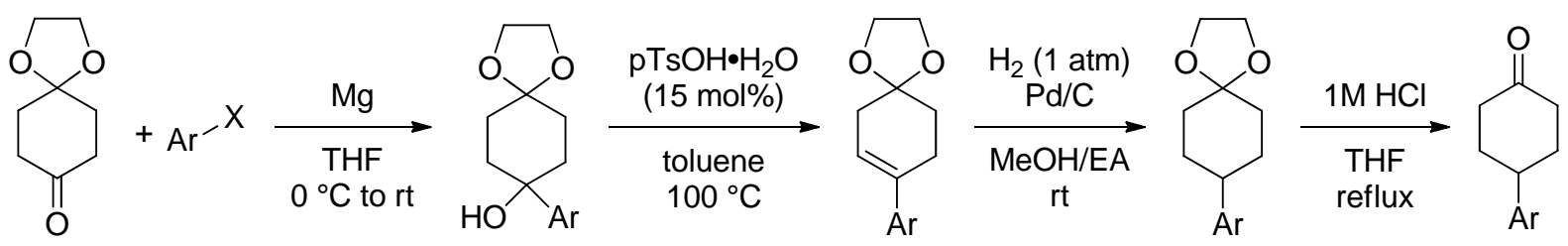

Note: All the intermediates can be isolated and purified by column chromatography using appropriate conditions. However, during our work we found that the dehydration step yielded a mixture of the desired acetal and the corresponding ketone, resulting from partial hydrolysis of the product. Therefore we found it sufficient to only purify after the Grignard addition and from there on rely only on a short aqueous work up after each step to carry all the material through to the final product. Ultimately the hydrolized side product will lead to the same desired cyclohexanone.

Method A: Purification after each step.

Method B: Purification only after the first and the last step.

Representative procedure (Method B):

8-(4-(tert-Butyl)phenyl)-1,4-dioxaspiro[4.5]decan-8-ol

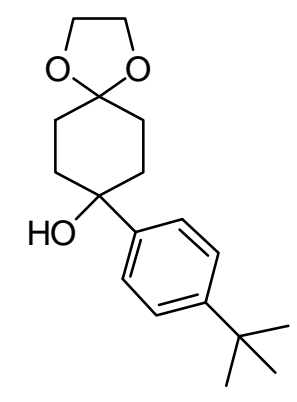

In a flame dried $100 \mathrm{~mL}$ three neck round bottom flask Mg (467 mg, 19.2 mmol) was layered with a minimum amount of anhydrous THF. After addition of 4-tert-butylbromobenzene (ca. $0.1 \mathrm{ml}$ ) and a few drops of 1,2dibromoethane the Grignard reaction was initiated by local heating (heat gun). After initiation the remaining THF (20 mL in total) and 4-tert-butylbromobenzene $(4.09 \mathrm{~g}, 19.2 \mathrm{mmol}$ in total) were added alternately to keep the reaction refluxing without the need for external heating. After complete addition the mixture was 
refluxed (oil bath heating) until the $\mathrm{Mg}$ was completely consumed. The Grignard solution was cooled to $0{ }^{\circ} \mathrm{C}$ and a solution of 1,4-dioxaspiro[4.5]decan-8-one (1.50 g, $\left.9.60 \mathrm{mmol}\right)$ in anhydrous THF (15 mL) was added over $30 \mathrm{~min}$. After complete addition, the cooling bath was removed and the mixture was stirred at ambient temperature until full conversion of 1,4-dioxaspiro[4.5]decan-8-one was observed (monitored by TLC). The reaction was quenched with a saturated solution of ammonium chloride $(5 \mathrm{~mL})$, the layers were separated and the aqueous layer was extracted with $\mathrm{CH}_{2} \mathrm{Cl}_{2}$. The combined organic layers were dried over $\mathrm{MgSO}_{4}$ and after evaporation of the solvent the residue was purified by column chromatography on $\mathrm{SiO}_{2}$ eluting with hexane:EtOAc (2:1) to give the product $2.57 \mathrm{~g}$ (8.85 mmol, 92\%) as a colorless solid.

${ }^{1} \mathrm{H}-\mathrm{NMR}\left(500 \mathrm{MHz}, \mathrm{CDCl}_{3}\right) \delta=7.46-7.44(\mathrm{~m}, 2 \mathrm{H})$, 7.38-7.35 (m, 2H), 4.04-3.95 (m, 4H), 2.20$2.07(\mathrm{~m}, 4 \mathrm{H}), 1.83-1.81(\mathrm{~m}, 2 \mathrm{H}), 1.75-1.68(\mathrm{~m}, 2 \mathrm{H}), 1.32(\mathrm{~s}, 9 \mathrm{H}) \mathrm{ppm}$.

${ }^{13} \mathrm{C}-\mathrm{NMR}\left(125 \mathrm{MHz}, \mathrm{CDCl}_{3}\right) \delta=149.9,145.5,125.3,124.4,108.6,72.3,64.5,64.4,36.7,34.5$, 31.5, 30.9 ppm.

MS (EI) m/z (\%): 290 (1), 230 (7), 215 (13), 191 (9), 176 (15), 161 (73), 133 (16), 101 (100), 86 (26).

HRMS $(\mathrm{ESI}+) \mathrm{m} / \mathrm{z}$ calculated for $\mathrm{C}_{18} \mathrm{H}_{26} \mathrm{O}_{3} \mathrm{Na}\left(\mathrm{M}+\mathrm{Na}^{+}\right)$313.177418, found 313.177143.

4-(4-(tert-Butyl)phenyl)cyclohexanone<smiles>CC(C)(C)c1ccc(C2CCC(=O)CC2)cc1</smiles>
A mixture of 8-(4-(tert-butyl)phenyl)-1,4-dioxaspiro[4.5]decan-8-ol (2.54 g, 8.76 $\mathrm{mmol})$ and $p \mathrm{TsOH} \cdot \mathrm{H}_{2} \mathrm{O}(250 \mathrm{mg}, 15 \mathrm{~mol} \%)$ in toluene $(30 \mathrm{~mL})$ was heated to $100{ }^{\circ} \mathrm{C}$ until the starting material was fully converted (monitored by TLC). After cooling to room temperature, saturated $\mathrm{NaHCO}_{3}$-solution $(20 \mathrm{~mL})$ was added. The layers were separated, the aqueous layer was extracted with $\mathrm{CH}_{2} \mathrm{Cl}_{2}$ and the combined organic layers were dried over $\mathrm{MgSO}_{4}$ and evaporated to dryness.

The residue was taken up in a 1:1 mixture of $\mathrm{MeOH}$ and ethyl acetate $(30 \mathrm{~mL})$ and after addition of $\mathrm{Pd} / \mathrm{C}(904 \mathrm{mg}, 10 \mathrm{~mol} \%, 10 \% \mathrm{Pd}$, dry) the mixture was stirred under an 
atmosphere of $\mathrm{H}_{2}$ (balloon) until complete conversion was observed (monitored by TLC). The mixture was filtered over Celite, washed with ethyl acetate and evaporated to dryness.

The residue was dissolved in THF $(30 \mathrm{~mL})$ and heated to reflux together with $1 \mathrm{M} \mathrm{HCl}(10 \mathrm{~mL})$. After full conversion (monitored by TLC), the solution was cooled to room temperature and basified by saturated $\mathrm{NaHCO}_{3}$-solution. The layers were separated, the aqueous layer was extracted with $\mathrm{CH}_{2} \mathrm{Cl}_{2}$ and the combined organic layers were dried over $\mathrm{MgSO}_{4}$ and evaporated to dryness. The residue was dissolved in a small amount of $\mathrm{CH}_{2} \mathrm{Cl}_{2}$ and filtered over a plug of $\mathrm{SiO}_{2}$ eluting with hexane:EtOAc (9:1). After evaporation of the solvent the residue was recrystallized from hexane to yield the title compound $1.21 \mathrm{~g}(5.23 \mathrm{mmol}, 60 \%$ over 3 steps) as a colorless solid.

${ }^{1} \mathrm{H}-\mathrm{NMR}\left(500 \mathrm{MHz}, \mathrm{CDCl}_{3}\right) \delta=7.36-7.34(\mathrm{~m}, 2 \mathrm{H}), 7.20-7.17(\mathrm{~m}, 2 \mathrm{H}), 3.00(\mathrm{tt}, J=6.0,3.4 \mathrm{~Hz}$, $1 \mathrm{H}), 2.55-2.47(\mathrm{~m}, 4 \mathrm{H}), 2.26-2.20(\mathrm{~m}, 2 \mathrm{H}), 1.98-1.90(\mathrm{~m}, 2 \mathrm{H}), 1.32(\mathrm{~s}, 9 \mathrm{H}) \mathrm{ppm}$.

${ }^{13} \mathrm{C}-\mathrm{NMR}\left(125 \mathrm{MHz}, \mathrm{CDCl}_{3}\right) \delta=211.6,149.5,141.8,126.5,125.6,42.3,41.6,34.5,34.1$ ppm. MS (EI) m/z (\%): 230 (22), 215 (100), 145 (8), 117 (7).

HRMS (EI) $m / z$ calculated for $\mathrm{C}_{16} \mathrm{H}_{22} \mathrm{O} 230.167066$, found 230.167280 .

4-(4-Methoxyphenyl)cyclohexanone

OMe Method A. Hexane:EtOAc (5:1). Colorless solid $433 \mathrm{mg}(2.12 \mathrm{mmol}, 64 \%$ overall yield).

${ }^{1} \mathbf{H}-\mathbf{N M R}\left(500 \mathrm{MHz}, \mathrm{CD}_{2} \mathrm{Cl}_{2}\right) \delta=7.19-7.16(\mathrm{~m}, 2 \mathrm{H}), 6.87-6.84(\mathrm{~m}, 2 \mathrm{H}), 3.77(\mathrm{~s}, 3 \mathrm{H})$, $2.97(\mathrm{tt}, J=6.1,3.4 \mathrm{~Hz}, 1 \mathrm{H}), 2.50(\mathrm{ddd}, J=14.1,14.0,6.0 \mathrm{~Hz}, 2 \mathrm{H}), 2.43-2.39(\mathrm{~m}, 2 \mathrm{H})$, 2.19-2.14 (m, 2H), 1.88 (ddd, $J=25.6,13.2,4.4 \mathrm{~Hz}, 2 \mathrm{H}$ ) ppm.

${ }^{13}$ C-NMR $\left(125 \mathrm{MHz}, \mathrm{CD}_{2} \mathrm{Cl}_{2}\right) \delta=210.9,158.6,137.6,127.9,114.1,55.5,42.2,41.7$, $34.6 \mathrm{ppm}$.

MS (EI) m/z (\%): 204 (55), 147 (100), 134 (51), 121 (12), 103 (8), 91 (23).

HRMS (EI) $m / z$ calculated for $\mathrm{C}_{13} \mathrm{H}_{16} \mathrm{O}_{2} 204.115030$, found 204.115038 . 


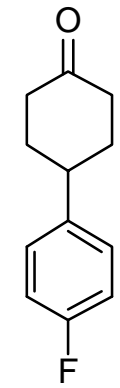

Method A. Purification: Hexane:EtOAc (6:1). Colorless solid 489 mg (2.54 mmol, 42\% overall yield).

${ }^{1} \mathrm{H}-\mathrm{NMR}\left(500 \mathrm{MHz}, \mathrm{CDCl}_{3}\right) \delta=7.23(\mathrm{dd}, J=8.2,5.6 \mathrm{~Hz}, 2 \mathrm{H}), 7.01$ (dd, $J=8.7,8.7 \mathrm{~Hz}$, $2 \mathrm{H}), 3.06-3.00(\mathrm{~m} 1 \mathrm{H}), 2.54-2.47(\mathrm{~m}, 2 \mathrm{H}), 2.44-2.41(\mathrm{~m}, 2 \mathrm{H}), 2.19-2.16(\mathrm{~m}, 2 \mathrm{H}), 1.89$ (dd, $J=25.8,13.0,4.1 \mathrm{~Hz}, 2 \mathrm{H}$ ) ppm.

${ }^{13} \mathrm{C}-\mathrm{NMR}\left(125 \mathrm{MHz}, \mathrm{CDCl}_{3}\right) \delta=210.6,161.8$ (d, $\left.J=243.6 \mathrm{~Hz}\right), 141.3(\mathrm{~d}, J=3.1 \mathrm{~Hz}), 128.5$ (d, $J$ $=7.7 \mathrm{~Hz}), 115.5(\mathrm{~d}, J=21.2 \mathrm{~Hz}), 42.3,41.6,34.5 \mathrm{ppm}$.

MS (EI) m/z (\%): 192 (96), 148 (12), 135 (60), 122 (100), 109 (68).

HRMS (EI) $m / z$ calculated for $\mathrm{C}_{12} \mathrm{H}_{13} \mathrm{FO} 192.095044$, found 192.094869.

4-(3,5-Dimethylphenyl)cyclohexanone<smiles>Cc1cc(C)cc(C2CCC(=O)CC2)c1</smiles>

Method A. Purification: Hexane:EtOAc (9:1). Colorless solid 336 mg (1.66 mmol, $26 \%$ overall yield).

${ }^{1} \mathrm{H}-\mathrm{NMR}\left(400 \mathrm{MHz}, \mathrm{CD}_{2} \mathrm{Cl}_{2}\right) \delta=6.87-6.85(\mathrm{~m}, 3 \mathrm{H}), 2.93(\mathrm{tt}, J=6.1,3.4 \mathrm{~Hz}, 1 \mathrm{H})$, $2.50(\mathrm{~m}, 2 \mathrm{H}), 2.44-2.39(\mathrm{~m}, 2 \mathrm{H}), 2.29(\mathrm{~s}, 6 \mathrm{H}), 2.20-2.12(\mathrm{~m}, 2 \mathrm{H}), 1.90(\mathrm{ddd}, J=$ 25.4, 13.1, 4.7 Hz, 2H) ppm.

${ }^{13} \mathrm{C}-\mathrm{NMR}\left(100 \mathrm{MHz}, \mathrm{CD}_{2} \mathrm{Cl}_{2}\right) \delta=211.0,145.5,138.3,128.3,124.9,43.0,41.7,34.4,21.4$ ppm. MS (EI) m/z (\%): 202 (79), 187 (5), 173 (22), 159 (9), 145 (54), 132 (100), 117 (32), 105 (14), $91(29)$.

HRMS (EI) $m / z$ calculated for $\mathrm{C}_{14} \mathrm{H}_{18} \mathrm{O} 202.135762$, found 202.135792 . 
4-(3,5-Dimethoxyphenyl)cyclohexanone<smiles>COc1cc(OC)cc(C2CCC(=O)CC2)c1</smiles>

Method B. Purification: Hexane:EtOAc (9:1). Colorless solid 1.24 g (5.29 $\mathrm{mmol}, 53 \%$ overall yield).

${ }^{1} \mathrm{H}-\mathrm{NMR}\left(500 \mathrm{MHz}, \mathrm{CD}_{2} \mathrm{Cl}_{2}\right) \delta=6.40(\mathrm{~d}, J=2.3 \mathrm{~Hz}, 2 \mathrm{H}), 6.32(\mathrm{~d}, J=2.3 \mathrm{~Hz}$, $2 \mathrm{H}), 3.77(\mathrm{~s}, 6 \mathrm{H}), 2.95(\mathrm{tt}, J=6.1,3.4 \mathrm{~Hz}, 1 \mathrm{H}), 2.52-2.46(\mathrm{~m}, 2 \mathrm{H}), 2.44-$ $2.39(\mathrm{~m}, 2 \mathrm{H}), 2.21-2.15(\mathrm{~m}, 2 \mathrm{H}), 1.90$ (ddd, $J=25.6,13.1,4.5 \mathrm{~Hz}, 2 \mathrm{H}) \mathrm{ppm}$.

${ }^{13}$ C-NMR $\left(125 \mathrm{MHz}, \mathrm{CD}_{2} \mathrm{Cl}_{2}\right) \delta=210.8,161.3,148.0,105.2,98.3,55.6,43.4,41.6,34.2$ ppm. MS (EI) m/z (\%): 234 (86), 191 (6), 177 (22), 164 (100), 152 (16).

HRMS $(E S I+) ~ m / z$ calculated for $\mathrm{C}_{14} \mathrm{H}_{18} \mathrm{O}_{3} \mathrm{Na}\left(\mathrm{M}+\mathrm{Na}^{+}\right)$257.114813, found 257.114721 .

4-(3-Chlorophenyl)cyclohexanone<smiles>O=C1CCC(c2cccc(Cl)c2)CC1</smiles>

Method B. Purification: Hexane:EtOAc (15:1). Colorless solid 466 mg (2.23 mmol, $23 \%$ overall yield).

${ }^{1}$ H-NMR $\left(500 \mathrm{MHz}, \mathrm{CDCl}_{3}\right) \delta=7.29-7.28(\mathrm{~m}, 1 \mathrm{H})$, 7.27-7.25 (m, 1H), 7.24-7.22 (m, $1 \mathrm{H}), 7.15(\mathrm{~d}, J=7.5 \mathrm{~Hz}, 1 \mathrm{H}), 3.02(\mathrm{tt}, J=6.1,3.4 \mathrm{~Hz}, 1 \mathrm{H}), 2.54-2.51(\mathrm{~m}, 4 \mathrm{H}), 2.26-$ $2.20(\mathrm{~m}, 2 \mathrm{H}), 2.00-1.89(\mathrm{~m}, 2 \mathrm{H}) \mathrm{ppm}$.

${ }^{13}$ C-NMR $\left(125 \mathrm{MHz}, \mathrm{CDCl}_{3}\right) \delta=210.8,146.9,134.5,130.0,127.1,126.9,125.0,42.6,41.3$, $33.9 \mathrm{ppm}$.

MS (EI) m/z (\%): 208 (100), 179 (21), 164 (6), 153 (55), 138 (62), 125 (49), 115 (33), 103 (20).

HRMS (EI) $m / z$ calculated for $\mathrm{C}_{12} \mathrm{H}_{13} \mathrm{ClO} 208.065490$, found 208.065299 . 
4-(Naphthalen-2-yl)cyclohexanone

1

Method B. Purification: Hexane:EtOAc (6:1). Colorless solid $1.43 \mathrm{~g}(6.38 \mathrm{mmol}$, $64 \%$ overall yield).

${ }^{1}$ H-NMR $\left(500 \mathrm{MHz} \mathrm{CDCl}_{3}\right) \delta=7.84-7.79(\mathrm{~m}, 3 \mathrm{H}), 7.69(\mathrm{~s}, 1 \mathrm{H})$, 7.50-7.44 (m, 2H), $7.39(\mathrm{dd}, J=8.5,1.8 \mathrm{~Hz}, 1 \mathrm{H}), 3.18(\mathrm{tt}, J=6.0-3.4 \mathrm{~Hz}, 1 \mathrm{H}), 2.61-2.52(\mathrm{~m}, 4 \mathrm{H}), 2.35-$ $2.29(\mathrm{~m}, 2 \mathrm{H}), 2.10-2.01(\mathrm{~m}, 2 \mathrm{H}) \mathrm{ppm}$.

${ }^{13} \mathrm{C}-\mathrm{NMR}\left(125 \mathrm{MHz}, \mathrm{CDCl}_{3}\right) \delta=211.3,142.3,133.7,132.5,128.3,127.7,127.7,126.3,125.7$, $125.7,124.9,42.9,41.5,34.0$ ppm.

MS (EI) m/z (\%): 224 (100), 195 (6), 167 (64), 154 (72), 141 (12), 128 (10).

HRMS (EI) $m / z$ calculated for $\mathrm{C}_{16} \mathrm{H}_{16} \mathrm{O} 224.120115$, found 224.119909 .

\section{4-Cyclohexylcyclohexanone}<smiles>O=C1CCC(C2CCCCC2)CC1</smiles>

To a solution of 4-cyclohexylcyclohexanol $(729 \mathrm{mg}, 4.0 \mathrm{mmol})$ in $\mathrm{CH}_{2} \mathrm{Cl}_{2}(20 \mathrm{~mL})$ were added MS $4 \AA ̊$ (2.8 g) and pyridinium chlorochromate (PCC) $(2.16 \mathrm{~g}, 10 \mathrm{mmol})$. After stirring at room temperature for $1.5 \mathrm{~h}$ Florisil was added and strring was continued for $10 \mathrm{~min}$. The mixture was filtered over a pad of Florisil and washed with $\mathrm{CH}_{2} \mathrm{Cl}_{2}$. After removal of the solvent the residue was purified by column chromatography on $\mathrm{SiO}_{2}$ eluting with hexane:EtOAC $(9: 1)$ to yield the title compound $595 \mathrm{mg}(3.30 \mathrm{mmol}, 83 \%)$ as colorless solid.

${ }^{1} \mathrm{H}-\mathrm{NMR}\left(400 \mathrm{MHz}, \mathrm{CDCl}_{3}\right) \delta=2.40-2.25(\mathrm{~m}, 4 \mathrm{H}), 2.03-1.99(\mathrm{~m}, 2 \mathrm{H}), 1.77-1.61(\mathrm{~m}, 5 \mathrm{H}), 1.57-$ $1.41(\mathrm{~m}, 3 \mathrm{H}), 1.28-1.07(\mathrm{~m}, 4 \mathrm{H}), 1.07-0.95(\mathrm{~m}, 2 \mathrm{H}) \mathrm{ppm}$.

${ }^{13} \mathrm{C}-\mathrm{NMR}\left(100 \mathrm{MHz}, \mathrm{CDCl}_{3}\right) \delta=212.8,42.1,41.9,41.3,30.5,29.9,26.8,26.7 \mathrm{ppm}$. 
MS (EI) m/z (\%): 180 (25), 162 (11), 151 (45), 133 (11), 125 (37), 109 (20), 98 (28), 83 (59), 67 (34), 55 (100), 41 (69).

HRMS (EI) $m / z$ calculated for $\mathrm{C}_{12} \mathrm{H}_{20} \mathrm{O} 180.151414$, found 180.151590 .

\section{N-Phthalimido-4-aminocyclohexanone}

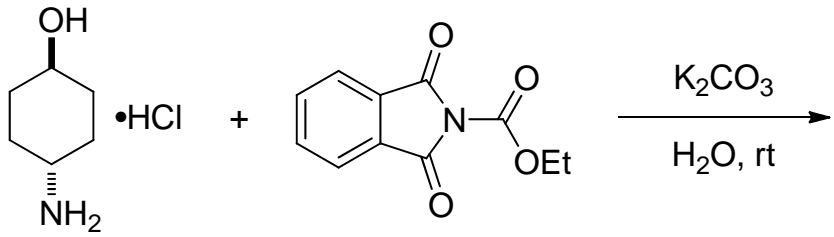<smiles>O=C1c2ccccc2C(=O)N1C1CCC(O)CC1</smiles>
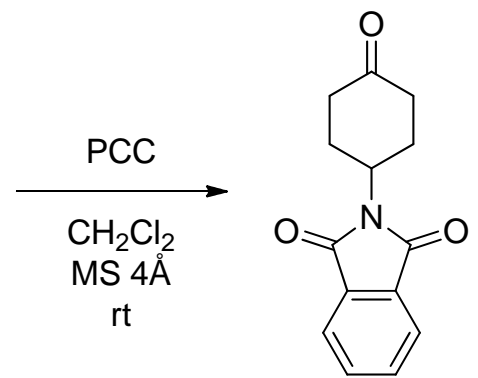

$\mathrm{N}$-Phthalimido-4-aminocyclohexanone was prepared from trans-4-aminocyclohexanol hydrochloride following a literature reported procedure. ${ }^{4}$

$N$-Phthalimido-trans-4-aminocyclohexanol<smiles>O=C1c2ccccc2C(=O)N1C1CCC(O)CC1</smiles>

To a solution of trans-4-aminocyclohexanol hydrochloride $(2.27 \mathrm{~g}, 15 \mathrm{mmol})$ in $\mathrm{H}_{2} \mathrm{O}(40 \mathrm{~mL})$ were added $\mathrm{K}_{2} \mathrm{CO}_{3}(5.72 \mathrm{~g}, 41.4 \mathrm{mmol})$ and $\mathrm{N}$-carbethoxy phthalimide $(3.69 \mathrm{~g}, 16.8 \mathrm{mmol})$. The mixture was stirred at room temperature for $30 \mathrm{~min}$ during which time the product precipitated. The solid was collected, washed with water and dried in vacuo to yield the title compound $3.07 \mathrm{~g}(12.5 \mathrm{mmol}, 84 \%)$ as a colorless solid.

${ }^{1} \mathrm{H}-\mathrm{NMR}\left(500 \mathrm{MHz}, \mathrm{CDCl}_{3}\right) \delta=7.83-7.79(\mathrm{~m}, 2 \mathrm{H}), 7.71-7.68(\mathrm{~m}, 2 \mathrm{H}), 4.12(\mathrm{tt}, J=6.2,4.0 \mathrm{~Hz}$, $1 \mathrm{H}), 3.75(\mathrm{tt}, J=5.5,4.4 \mathrm{~Hz}, 1 \mathrm{H}), 2.34(\mathrm{ddd}, J=25.9,12.2,3.5 \mathrm{~Hz}, 2 \mathrm{H}), 2.13-2.06(\mathrm{~m}, 2 \mathrm{H})$, 1.79-.172 (m, 2H), $1.63(\mathrm{br}, 1 \mathrm{H}), 1.44$ (ddd, $J=24.3,13.2,3.4 \mathrm{~Hz}, 2 \mathrm{H}) \mathrm{ppm}$.

${ }^{13} \mathrm{C}-\mathrm{NMR}\left(125 \mathrm{MHz}, \mathrm{CDCl}_{3}\right) \delta=168.5,134.0,132.1,123.2,69.7,49.8,39.9,34.9,27.7 \mathrm{ppm}$. MS (EI) m/z (\%): 245 (75), 227 (12), 212 (25), 187 (25), 173 (17), 148 (100), 130 (64), 104 (38), $76(48)$.

HRMS $(E S I+) \mathrm{m} / \mathrm{z}$ calculated for $\mathrm{C}_{14} \mathrm{H}_{15} \mathrm{NO}_{3} \mathrm{Na}\left(\mathrm{M}+\mathrm{Na}^{+}\right)$268.094410, found 268.094135 .

(4) Glennon, R. A.; Hong, S.-S.; Bondarev, M.; Law, H.; Dukat, M.; Rakhit, S.; Power, P.; Fan, E.; Kinneau, D.; Kamboj, R.; Teitler, M.; Herrick-Davis, K.; Smith C. J. Med. Chem. 1996, 39, 314. 
$N$-Phthalimido-4-aminocyclohexanone<smiles>O=C1CCC(N2C(=O)c3ccccc3C2=O)CC1</smiles>

To a solution of $N$-phthalimido-trans-4-aminocyclohexanol (1.00 g, $4.08 \mathrm{mmol})$ in $\mathrm{CH}_{2} \mathrm{Cl}_{2}(20 \mathrm{~mL})$ were added MS $4 \AA$ ( $\left.2.8 \mathrm{~g}\right)$ and PCC $(2.22 \mathrm{~g}, 10.3 \mathrm{mmol})$. After stirring at room temperature for $1.5 \mathrm{~h}$ Florisil was added and stirring was continued for $10 \mathrm{~min}$. The mixture was filtered over a pad of Florisil and washed with $\mathrm{CH}_{2} \mathrm{Cl}_{2}$. After removal of the solvent the residue was purified by column chromatography on $\mathrm{SiO}_{2}$ eluting with hexane:EtOAc $(2: 1)$ to yield the title compound $752 \mathrm{mg}$ (3.09 mmol, 76\%) as colorless solid.

${ }^{1} \mathrm{H}-\mathrm{NMR}\left(500 \mathrm{MHz}, \mathrm{CDCl}_{3}\right) \delta=7.84-7.81(\mathrm{~m}, 2 \mathrm{H}), 7.73-7.71(\mathrm{~m}, 2 \mathrm{H}), 4.61(\mathrm{tt}, J=6.0,4.0 \mathrm{~Hz}$, $1 \mathrm{H}), 2.71(\mathrm{ddd}, J=24.8,12.4,5.9 \mathrm{~Hz}, 2 \mathrm{H}), 2.55-2.45(\mathrm{~m}, 4 \mathrm{H}), 2.09-2.01(\mathrm{~m}, 2 \mathrm{H}) \mathrm{ppm}$.

${ }^{13}$ C-NMR $\left(125 \mathrm{MHz}, \mathrm{CDCl}_{3}\right) \delta=209.0,168.2,134.2,131.9,123.4,48.4,40.0,28.7$ ppm.

MS (EI) m/z (\%): 186 (8), 173 (8), 148 (13), 130 (11), 104 (12), 96 (100).

HRMS (ESI+) $m / z$ calculated for $\mathrm{C}_{14} \mathrm{H}_{13} \mathrm{NO}_{3} \mathrm{Na}\left(\mathrm{M}+\mathrm{Na}^{+}\right)$266.078764, found 266. 078360.

\section{cis-3,4-Diphenyl-cyclopentanone}<smiles>CC(=O)COC(C)=O</smiles><smiles>O=C1C=C(c2ccccc2)C(O)(c2ccccc2)C1</smiles>

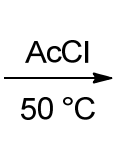<smiles>O=C1C=C(c2ccccc2)C(Cl)(c2ccccc2)C1</smiles><smiles>CCO[R10]#[Te]</smiles><smiles>O=C1CC(c2ccccc2)=C(c2ccccc2)C1</smiles><smiles>O=C1CC(c2ccccc2)C(c2ccccc2)C1</smiles>

The synthesis of cis-3,4-diphenyl-cyclopentanone was accomplished by using modified literature reported procedures. ${ }^{5,6,7}$

(5) Arriola, D. J.; Bokota, M.; Campbell, R. E., Jr.; Klosin, J.; LaPointe, R. E.; Redwine, O. D.; Shankar, R. B.; Timmers, F. J.; Abboud, K. A. J. Am. Chem. Soc. 2007, 129, 7065.
(6) Japp, F. R.; Knox, J. J. Chem. Soc., Trans. 1905, 87, 673.

(7) Corey, E. J.; Uda, H. J. Am. Chem. Soc. 1963, 85, 1788. 
4-Hydroxy-3,4-diphenylcyclopent-2-enone $\mathrm{e}^{5,6}$

$\overbrace{\mathrm{Ph}}^{\mathrm{OH}}$

To a mixture of benzil $(5.0 \mathrm{~g}, 23.8 \mathrm{mmol})$ and acetone $(3.2 \mathrm{~g}, 55 \mathrm{mmol})$ was added $0.1 \mathrm{~mL}$ of $32 \%$ aqueous solution of $\mathrm{KOH}$. After stirring at room temperature for $5 \mathrm{~min}$, another $2 \mathrm{~mL}$ of $32 \%$ solution of $\mathrm{KOH}$ was added and the mixture was heated to reflux for $1 \mathrm{~h}$. The resulting brown oil was allowed to cool to room temperature and was then poured into $\mathrm{H}_{2} \mathrm{O}$ and extracted with $\mathrm{CH}_{2} \mathrm{Cl}_{2}$. The combined organic layers were dried over $\mathrm{MgSO}_{4}$, evaporated to dryness and the residue was crystallized from boiling toluene to yield the product $3.94 \mathrm{~g}(15.8 \mathrm{mmol}, 66 \%)$ as a yellow solid.

${ }^{1} \mathrm{H}-\mathrm{NMR}\left(500 \mathrm{MHz}, \mathrm{CDCl}_{3}\right) \delta=7.53-7.51(\mathrm{~m}, 2 \mathrm{H}), 7.46-7.44(\mathrm{~m}, 2 \mathrm{H})$, 7.37-7.34 (m, 3H), 7.31$7.27(\mathrm{~m}, 3 \mathrm{H}), 6.70(\mathrm{~s}, 1 \mathrm{H}), 3.02(\mathrm{~d}, J=18.5 \mathrm{~Hz}, 1 \mathrm{H}), 2.90(\mathrm{~d}, J=18.5 \mathrm{~Hz}, 1 \mathrm{H}), 2.70(\mathrm{br}, 1 \mathrm{H})$ ppm.

${ }^{13}$ C-NMR $\left(125 \mathrm{MHz}, \mathrm{CDCl}_{3}\right) \delta=204.9,174.0,144.2,131.4,131.0,129.3,129.3,129.0,129.0$, $127.7,124.3,81.8,56.7 \mathrm{ppm}$.

MS (EI) $m / z$ (\%): 250 (100), 222 (13), 147 (12), 131 (35), 105 (48), 77 (24).

HRMS (ESI+) $\mathrm{m} / z$ calculated for $\mathrm{C}_{17} \mathrm{H}_{14} \mathrm{O}_{2} \mathrm{Na}\left(\mathrm{M}+\mathrm{Na}^{+}\right)$273.088597, found 273.088864 .

4-Chloro-3,4-diphenylcyclopent-2-enone ${ }^{7}$<smiles>O=C1C=C(c2ccccc2)C(Cl)(c2ccccc2)C1</smiles>

A solution of 4-hydroxy-3,4-diphenylcyclopent-2-enone (1.06 g, $4.24 \mathrm{mmol}$ ) in $\mathrm{AcCl}(4 \mathrm{~mL})$ was heated to $50{ }^{\circ} \mathrm{C}$ for $14 \mathrm{~h}$. After cooling to room temperature the volatile components were removed under reduced pressure. The residue was taken up in toluene and the product was precipitated by adding hexane to this solution. The obtained solid was collected and washed with hexane to give the product $546 \mathrm{mg}(2.03 \mathrm{mmol}, 48 \%)$ as a colorless solid.

${ }^{1} \mathrm{H}-\mathrm{NMR}\left(500 \mathrm{MHz}, \mathrm{CD}_{2} \mathrm{Cl}_{2}\right) \delta=7.56-7.54(\mathrm{~m}, 2 \mathrm{H})$, 7.52-7.50 (m, 2H), 7.40-7.34 (m, 3H), 7.33$7.30(\mathrm{~m}, 3 \mathrm{H}), 6.86(\mathrm{~s}, 1 \mathrm{H}), 3.46(\mathrm{~d}, J=19.0 \mathrm{~Hz}, 1 \mathrm{H}), 3.07$ (d, J=19.0 Hz, 1H) ppm. 
${ }^{13} \mathrm{C}-\mathrm{NMR}\left(125 \mathrm{MHz}, \mathrm{CD}_{2} \mathrm{Cl}_{2}\right) \delta=202.6,172.6,142.5,131.3,131.2,130.2,129.8,129.2,128.8$, $128.4,125.9,74.2,59.7$ ppm.

MS (EI) m/z (\%): 268 (28), 233 (100), 205 (26), 102 (16).

HRMS (EI) $m / z$ calculated for $\mathrm{C}_{17} \mathrm{H}_{13} \mathrm{ClO} 268.065495$, found 268.065457 .

\section{3,4-Diphenylcyclopent-3-enone ${ }^{7}$}<smiles>O=C1CC(c2ccccc2)=C(c2ccccc2)C1</smiles>

A solution of 4-chloro-3,4-diphenylcyclopent-2-enone (540 $\mathrm{mg}, 2.01 \mathrm{mmol}$ ) in $\mathrm{Et}_{2} \mathrm{O}(15 \mathrm{~mL})$ was added to a vigorously stirred suspension of zinc dust (4.40 g, $67.3 \mathrm{mmol})$ in $\mathrm{Et}_{2} \mathrm{O}(20 \mathrm{~mL})$ and $\mathrm{AcOH}(1 \mathrm{~mL})$. After complete addition the mixture was stirred for $30 \mathrm{~min}$ and then filtered over Celite and washed with $\mathrm{Et}_{2} \mathrm{O}$. The etheric layer was washed with $\mathrm{H}_{2} \mathrm{O}$ and saturated $\mathrm{NaHCO}_{3}$-solution, dried over $\mathrm{MgSO}_{4}$ and evaporated to dryness, to give the title compound $451 \mathrm{mg}(1.92 \mathrm{mmol}, 96 \%)$ as a colorless solid.

${ }^{1} \mathrm{H}-\mathrm{NMR}\left(500 \mathrm{MHz}, \mathrm{CD}_{2} \mathrm{Cl}_{2}\right) \delta=7.27-7.19(\mathrm{~m}, 10 \mathrm{H}), 3.46(\mathrm{~s}, 4 \mathrm{H})$.

${ }^{13} \mathrm{C}-\mathrm{NMR}\left(125 \mathrm{MHz}, \mathrm{CD}_{2} \mathrm{Cl}_{2}\right) \delta=213.8,137.0,134.8,128.6,128.5,127.8,49.3$ ppm.

MS (EI) m/z (\%): 234 (100), 205 (58), 191 (24), 128 (16), 91 (41).

HRMS (EI) $m / z$ calculated for $\mathrm{C}_{17} \mathrm{H}_{14} \mathrm{O} 234.104465$, found 234.104206.

cis-3,4-Diphenyl-cyclopentanone<smiles>O=C1CC(c2ccccc2)C(c2ccccc2)C1</smiles>

A mixture of 3,4-diphenylcyclopent-3-enone (449 mg, $1.92 \mathrm{mmol}$ ) and Pd/C (204 $\mathrm{mg}, 10 \mathrm{~mol} \%, 10 \% \mathrm{Pd}$, dry) in ethyl acetate $(10 \mathrm{~mL})$ was stirred under an atmosphere of $\mathrm{H}_{2}$ (balloon) for $4 \mathrm{~h}$. The mixture was filtered over Celite, washed with ethyl acetate and evaporated to dryness. The residue was purified by column chromatography on $\mathrm{SiO}_{2}$ eluting with hexane:EtOAc $(5: 1)$ to yield the title compound $410 \mathrm{mg}(1.74 \mathrm{mmol}, 90 \%)$ as a colorless solid. 
${ }^{1} \mathrm{H}-\mathrm{NMR}\left(500 \mathrm{MHz}, \mathrm{CD}_{2} \mathrm{Cl}_{2}\right) \delta=7.12-7.09(\mathrm{~m}, 6 \mathrm{H}), 6.79-6.76(\mathrm{~m}, 4 \mathrm{H}), 3.91-3.86(\mathrm{~m}, 2 \mathrm{H}), 2.75$ $(\mathrm{dd}, \mathrm{J}=18.9,6.7 \mathrm{~Hz}, 2 \mathrm{H}), 2.67(\mathrm{dd}, \mathrm{J}=18.7,8.2 \mathrm{~Hz}, 2 \mathrm{H}) \mathrm{ppm}$.

${ }^{13} \mathrm{C}-\mathrm{NMR}\left(125 \mathrm{MHz}, \mathrm{CD}_{2} \mathrm{Cl}_{2}\right) \delta=218.3,140.2,128.4,128.2,126.8,47.6,43.5 \mathrm{ppm}$.

MS (EI) $m / z$ (\%): 236 (36), 104 (100), 78 (8).

HRMS (EI) $m / z$ calculated for $\mathrm{C}_{17} \mathrm{H}_{16} \mathrm{O} 236.120118$, found 236.120141.

\section{N-Benzyl phenylhydrazines}

The $N$-benzylation of phenylhydrazines was accomplished following a literature reported protocol. $^{8}$

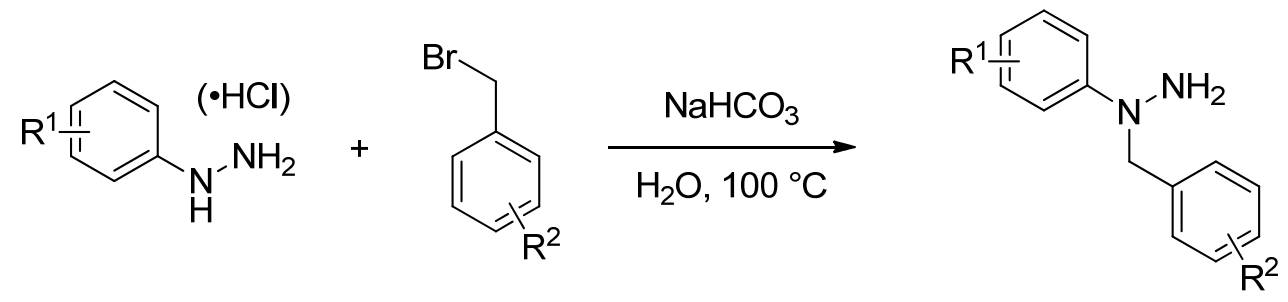

The hydrazine or its hydrochloride salt $(4.0 \mathrm{mmol})$, the corresponding benzyl bromide $(4.0$ $\mathrm{mmol})$ and $\mathrm{NaHCO}_{3}(4.0 \mathrm{mmol}$ for hydrazines, $8.0 \mathrm{mmol}$ for hydrochloride salts) were mixed in $\mathrm{H}_{2} \mathrm{O}(3-5 \mathrm{~mL})$ and heated to $100{ }^{\circ} \mathrm{C}$ under vigorous stirring. After $3 \mathrm{~h}$ the mixture was cooled to room temperature and diluted with $\mathrm{Et}_{2} \mathrm{O}(30 \mathrm{~mL})$. The layers were separated, the organic layer was dried over $\mathrm{MgSO}_{4}$ and concentrated under reduced pressure. The residue was purified by column chromatography on $\mathrm{SiO}_{2}$, using pentane: $\mathrm{Et}_{2} \mathrm{O}$ as the eluent.

1-Benzyl-1-phenylhydrazine<smiles>NN(Cc1ccccc1)c1ccccc1</smiles>

The reaction was conducted on a $8.0 \mathrm{mmol}$ scale.

Purification: Pentane: $\mathrm{Et}_{2} \mathrm{O}$ (3:1). Orange solid $833 \mathrm{mg}$ (4.20 mmol, 53\%).

${ }^{1} \mathrm{H}-\mathrm{NMR}\left(500 \mathrm{MHz}, \mathrm{CDCl}_{3}\right) \delta=7.36-7.27(\mathrm{~m}, 7 \mathrm{H}), 7.11(\mathrm{~d}, J=7.9 \mathrm{~Hz}, 2 \mathrm{H})$, $6.84(\mathrm{t}, J=7.3 \mathrm{~Hz}, 1 \mathrm{H}), 4.60(\mathrm{~s}, 2 \mathrm{H}), 3.74(\mathrm{br}, 2 \mathrm{H}) \mathrm{ppm}$.

(8) Perni, R. B.; Gribble, G. W. Org. Prep. Proced. Int. 1982, 14, 343. 
${ }^{13}$ C-NMR $\left(125 \mathrm{MHz}, \mathrm{CD}_{2} \mathrm{Cl}_{2}\right) \delta=151.6,137.5,129.2,128.8,128.1,127.6,119.1,114.1,60.6$ ppm.

MS (EI) m/z (\%): 198 (36), 107 (100), 91 (24), 77 (34), 65 (7).

HRMS (EI) $m / z$ calculated for $\mathrm{C}_{13} \mathrm{H}_{14} \mathrm{~N}_{2}$ 198.115696, found 198.115879 .

1-(4-lodobenzyl)-1-phenylhydrazine<smiles>NN(Cc1ccc(I)cc1)c1ccccc1</smiles>

Purification: Pentane: $\mathrm{Et}_{2} \mathrm{O}$ (3:1). Orange solid $788 \mathrm{mg}$ (2.43 mmol, 61\%). ${ }^{1}$ H-NMR $\left(500 \mathrm{MHz}, \mathrm{CDCl}_{3}\right) \delta=7.69-7.66(\mathrm{~m}, 2 \mathrm{H}), 7.27-7.22(\mathrm{~m}, 2 \mathrm{H}), 7.08$ $(\mathrm{d}, J=8.4 \mathrm{~Hz}, 2 \mathrm{H}), 7.04-7.02(\mathrm{~m}, 2 \mathrm{H}), 6.80(\mathrm{tt}, J=7.3,1.0 \mathrm{~Hz}, 1 \mathrm{H}), 4.54(\mathrm{~s}$, $2 \mathrm{H}), 3.61(\mathrm{br}, 2 \mathrm{H}) \mathrm{ppm}$.

${ }^{13} \mathrm{C}-\mathrm{NMR}\left(125 \mathrm{MHz}, \mathrm{CDCl}_{3}\right) \delta=152.0,138.2,137.9,130.2,129.3,118.8,113.7,92.7,60.0$ ppm.

MS (EI) m/z (\%):324 (22), 217 (11), 107 (100), 90 (10), 77 (21).

HRMS (EI) $m / z$ calculated for $\mathrm{C}_{13} \mathrm{H}_{13} \mathrm{~N}_{2} 1$ 324.012348, found 324.012027 .

1-(4-lodobenzyl)-1-(p-tolyl)hydrazine<smiles>Cc1ccc(N(N)Cc2ccc(I)cc2)cc1</smiles>

Purification: Hexane:EtOAc (7:1). Yellow solid $839 \mathrm{mg}(2.48 \mathrm{mmol}$, $62 \%)$.

${ }^{1} \mathrm{H}-\mathrm{NMR}\left(400 \mathrm{MHz}, \mathrm{CD}_{2} \mathrm{Cl}_{2}\right) \delta=7.68-7.65(\mathrm{~m}, 2 \mathrm{H})$, 7.09-7.03 $(\mathrm{m}$, 4H), 6.95-6.91 (m, 2H), $4.47(\mathrm{~s}, 2 \mathrm{H}), 3.55(\mathrm{br}, 2 \mathrm{H}), 2.25(\mathrm{~s}, 3 \mathrm{H}) \mathrm{ppm}$.

${ }^{13} \mathrm{C}-\mathrm{NMR}\left(100 \mathrm{MHz}, \mathrm{CD}_{2} \mathrm{Cl}_{2}\right) \delta=150.0,138.4,137.9,130.4,129.8,128.4,114.2,92.6,60.7$, $20.4 \mathrm{ppm}$.

MS (EI) $m / z$ (\%):338 (9), 217 (5), 121 (100), 91 (27), 77 (22).

HRMS $(E S I+) m / z$ calculated for $\mathrm{C}_{14} \mathrm{H}_{15} \mathrm{~N}_{2} \mathrm{INa}\left(\mathrm{M}+\mathrm{Na}^{+}\right)$361.017217, found 361.017179. 
1-(4-iodobenzyl)-1-(4-methoxyphenyl)hydrazine<smiles>COc1ccc(N(N)Cc2ccc(I)cc2)cc1</smiles>
The reaction was conducted on a $3.0 \mathrm{mmol}$ scale. Purification: Pentane: $\mathrm{Et}_{2} \mathrm{O}(4: 1)$ pale yellow solid $468 \mathrm{mg}(1.32$ $\mathrm{mmol}, 44 \%)$.

${ }^{1} \mathrm{H}-\mathrm{NMR}\left(500 \mathrm{MHz}, \mathrm{CDCl}_{3}\right) \delta=7.65-7.63(\mathrm{~m}, 2 \mathrm{H}), 7.05(\mathrm{~d}, \mathrm{~J}=8.2$ $\mathrm{Hz}, 2 \mathrm{H}), 7.01-7.00(\mathrm{~m}, 2 \mathrm{H}), 6.83-6.82(\mathrm{~m}, 2 \mathrm{H}), 4.37(\mathrm{~s}, 2 \mathrm{H}), 3.76,(\mathrm{~s}, 3 \mathrm{H}) \mathrm{ppm}$.

${ }^{13}$ C-NMR $\left(125 \mathrm{MHz}, \mathrm{CDCl}_{3}\right) \delta=153.6,146.0,137.7,137.3,130.3,116.4,114.5,92.8,61.8$, $55.7 \mathrm{ppm}$.

MS (EI) m/z (\%): 354 (15), 217 (7), 137 (100).

HRMS (EI): $m / z$ calculated for $\mathrm{C}_{14} \mathrm{H}_{15} \mathrm{~N}_{2} \mathrm{OI} 354.022909$, found 354.023184 .

1-(4-bromophenyl)-1-(4-iodobenzyl)hydrazine<smiles>NN(Cc1ccc(I)cc1)c1ccc(Br)cc1</smiles>
The reaction was conducted on a $4.0 \mathrm{mmol}$ scale. Purification: Pentane:Et ${ }_{2} \mathrm{O}$ (4:1) pale brown solid $548 \mathrm{mg}(1.83$ $\mathrm{mmol}, 34 \%)$.

${ }^{1} \mathbf{H}-\mathbf{N M R}\left(500 \mathrm{MHz}, \mathrm{CDCl}_{3}\right) \delta=7.68-7.59(\mathrm{~m}, 2 \mathrm{H}), 7.30(\mathrm{~d}, \mathrm{~J}=8.8 \mathrm{~Hz}$, $2 \mathrm{H}), 7.00(\mathrm{~d}, \mathrm{~J}=8.1 \mathrm{~Hz}, 2 \mathrm{H}), 6.90(\mathrm{~d}, \mathrm{~J}=8.8 \mathrm{~Hz}, 2 \mathrm{H}), 4.49(\mathrm{~s}, 2 \mathrm{H}), 3.54$ (s (broad), 2H) ppm.

${ }^{13}$ C-NMR $\left(125 \mathrm{MHz}, \mathrm{CDCl}_{3}\right) \delta=150.6,138.0,136.9,131.9,129.8,115.3,110.9,93.0,59.8$ ppm.

MS (EI) m/z (\%): 404 (20), 402 (20), 217 (30), 187 (100), 185 (100), 90 (35), 77 (50).

HRMS (EI): $m / z$ calculated for $\mathrm{C}_{13} \mathrm{H}_{12} \mathrm{~N}_{2} \mathrm{Brl} 401.922871$, found 401.922948 . 
1-(3,5-Dimethylphenyl)-1-(4-iodobenzyl)hydrazine<smiles>Cc1cc(C)cc(N(N)Cc2ccc(I)cc2)c1</smiles>

The reaction was conducted on a $3.0 \mathrm{mmol}$ scale.

Purification: Pentane:Et ${ }_{2} \mathrm{O}$ (4:1) pale brown solid $685 \mathrm{mg}(1.94 \mathrm{mmol}$, $65 \%)$.

${ }^{1} \mathrm{H}-\mathrm{NMR}\left(500 \mathrm{MHz}, \mathrm{CDCl}_{3}\right) \delta=7.67(\mathrm{~d}, J=8.2 \mathrm{~Hz}, 2 \mathrm{H}), 7.07(\mathrm{~d}, J=8.2 \mathrm{~Hz}$, 2H), $6.68(\mathrm{~s}, 2 \mathrm{H}), 6.51(\mathrm{~s}, 1 \mathrm{H}), 4.51(\mathrm{~s}, 2 \mathrm{H}), 3.56$ (s (broad), 2H), 2.29 (s, 6H) ppm.

${ }^{13} \mathrm{C}-\mathrm{NMR}\left(100 \mathrm{MHz}, \mathrm{CDCl}_{3}\right) \delta=152.1,139.1,138.1,138.0,130.1,121.2,111.7,92.9,60.2$, $22.0 \mathrm{ppm}$.

MS (EI) m/z (\%): 352 (24), 217 (9), 135 (100).

HRMS (EI) $m / z$ calculated for $\mathrm{C}_{15} \mathrm{H}_{17} \mathrm{~N}_{2} \mathrm{I}\left(\mathrm{M}+\mathrm{Na}^{+}\right)$352.043646, found 352.043555 .

1-(4-lodobenzyl)-1-(m-tolyl)hydrazine<smiles>Cc1cccc(N(N)Cc2ccc(I)cc2)c1</smiles>

The reaction was conducted on a $3.0 \mathrm{mmol}$ scale.

Purification: Pentane:Et ${ }_{2} \mathrm{O}$ (4:1) pale brown solid $497 \mathrm{mg}(1.47 \mathrm{mmol}$, 49\%)

${ }^{1} \mathrm{H}-\mathrm{NMR}\left(500 \mathrm{MHz}, \mathrm{CDCl}_{3}\right) \delta=7.68-7.66(\mathrm{~m}, 2 \mathrm{H}), 7.17(\mathrm{~d}, J=7.8,7.8 \mathrm{~Hz}$, 1H), 7.08-7.06 (m, 2H), $6.90(\mathrm{~s}, 1 \mathrm{H}), 6.86-6.83(\mathrm{~m}, 1 \mathrm{H}), 6.68(\mathrm{~d}, J=7.8 \mathrm{~Hz}, 1 \mathrm{H}), 4.53(\mathrm{~s}, 2 \mathrm{H})$, 3.67 (s (broad), 2H), 2.34 (s, 3H) ppm.

${ }^{13} \mathrm{C}-\mathrm{NMR}\left(100 \mathrm{MHz}, \mathrm{CDCl}_{3}\right) \delta=151.6,138.9,138.7,137.6,129.8,128.9,119.6,113.4,110.7$, 92.5, 60.9, $21.8 \mathrm{ppm}$.

MS (EI) m/z (\%): 338 (25), 217 (8), 121 (100), 91 (22), 77 (18).

HRMS (EI) $m / z$ calculated for $\mathrm{C}_{14} \mathrm{H}_{15} \mathrm{~N}_{2} \mathrm{INa}$ : 361.017212, found 361.017037. 


\section{Hydrazones}

General procedure for the synthesis of hydrazones 1a-1u:<smiles>[X]c1ccc(CN(N=C2CCC([R])CC2)c2ccccc2)cc1</smiles>

A mixture of the benzyl protected phenylhydrazine $(0.50 \mathrm{mmol})$, the corresponding ketone $(0.50 \mathrm{mmol})$ and one drop of acetic acid in EtOH $(1.0 \mathrm{~mL})$ was stirred at $60^{\circ} \mathrm{C}$ for $3 \mathrm{~h}$. After full conversion of the starting materials (monitored by TLC), the mixture was slowly cooled to $-20{ }^{\circ} \mathrm{C}$. The precipitate was filtered, washed with EtOH and dried in vacuo to yield the corresponding hydrazone.

1-Benzyl-1-phenyl-2-(4-phenylcyclohexylidene)hydrazine (1a)<smiles>c1ccc(CN(N=C2CCC(c3ccccc3)CC2)c2ccccc2)cc1</smiles>

1a

The reaction was conducted on a $2.0 \mathrm{mmol}$ scale.

Colorless solid $612 \mathrm{mg}$ (1.73 mmol, 86\%).

${ }^{1} \mathbf{H}-\mathbf{N M R}\left(500 \mathrm{MHz}, \mathrm{CD}_{2} \mathrm{Cl}_{2}\right) \delta=7.37(\mathrm{~d}, J=7.1 \mathrm{~Hz}, 2 \mathrm{H}), 7.33$ (dd, $J$ $=7.8,7.3 \mathrm{~Hz}, 2 \mathrm{H}), 7.29-7.21(\mathrm{~m}, 5 \mathrm{H}), 7.18(\mathrm{tt}, J=7.2,1.0 \mathrm{~Hz}, 1 \mathrm{H})$, $7.13(\mathrm{~d}, J=7.2 \mathrm{~Hz}, 2 \mathrm{H}), 6.89-6.87(\mathrm{~m}, 2 \mathrm{H}), 6.84(\mathrm{t}, J=7.3 \mathrm{~Hz}, 1 \mathrm{H})$, $4.66(\mathrm{~d}, J=14.9 \mathrm{~Hz}, 1 \mathrm{H}), 4.53(\mathrm{~d}, J=14.9 \mathrm{~Hz}, 1 \mathrm{H}), 3.18-3.13(\mathrm{~m}$, 1H), 2.78-2.69 (m, 2H), 2.40 (ddd, $J=13.7,13.7,5.1 \mathrm{~Hz}, 1 \mathrm{H}), 2.09-2.04(\mathrm{~m}, 1 \mathrm{H}), 1.82-1.78(\mathrm{~m}$, 1H), 1.71 (ddd, $J=13.8,13.6,6.6 \mathrm{~Hz}, 1 \mathrm{H}$ ), 1.62 (ddd, $J=25.6,13.1,4.0 \mathrm{~Hz}, 1 \mathrm{H}$ ), 1.15 (ddd, $J=$ 25.6, 13.0, $4.1 \mathrm{~Hz}, 1 \mathrm{H}) \mathrm{ppm}$.

${ }^{13}$ C-NMR $\left(125 \mathrm{MHz}, \mathrm{CD}_{2} \mathrm{Cl}_{2}\right) \delta=176.2,150.9,146.2,139.4,129.1,129.0,128.7,128.6,127.2$, $127.0,126.5,120.0,116.0,60.5,43.9,35.3,34.8,33.5,29.7$ ppm.

MS (EI) m/z (\%): 354 (100), 263 (100), 235 (8), 159 (14), 144 (41), 117 (15), 91 (37), 77 (36).

HRMS (EI) $m / z$ calculated for $\mathrm{C}_{25} \mathrm{H}_{26} \mathrm{~N}_{2} 354.209599$, found 354.209233. 


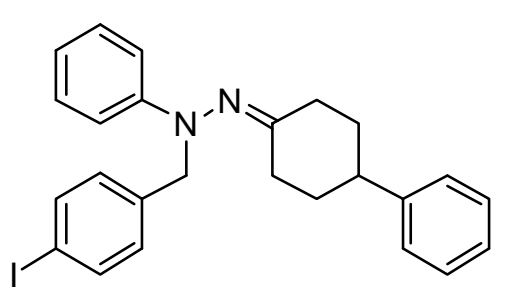

1b

The reaction was conducted on a $1.0 \mathrm{mmol}$ scale. Colorless solid $410 \mathrm{mg}$ (0.853 mmol, 85\%).

${ }^{1} \mathrm{H}-\mathrm{NMR}\left(500 \mathrm{MHz}, \mathrm{CD}_{2} \mathrm{Cl}_{2}\right) \delta=7.71-7.68(\mathrm{~m}, 2 \mathrm{H})$ ) 7.34-7.31 $(\mathrm{m}, 2 \mathrm{H}), 7.28-7.24(\mathrm{~m}, 2 \mathrm{H}), 7.23-7.19(\mathrm{~m}, 1 \mathrm{H}), 7.18-7.15(\mathrm{~m}$, $4 \mathrm{H}), 6.90-6.87(\mathrm{~m}, 3 \mathrm{H}), 4.63(\mathrm{~d}, J=14.8 \mathrm{~Hz}, 1 \mathrm{H}), 4.46(\mathrm{~d}, J=$ $14.8 \mathrm{~Hz}, 1 \mathrm{H}), 3.17-3.13(\mathrm{~m}, 1 \mathrm{H}), 2,80-2,69(\mathrm{~m}, 2 \mathrm{H}), 2.41$ (ddd, $J=13.7,13.7,5.1 \mathrm{~Hz}, 1 \mathrm{H}), 2.11-2.06(\mathrm{~m}, 1 \mathrm{H}), 1.84-1.80(\mathrm{~m}, 1 \mathrm{H}), 1.71-1.70(\mathrm{~m}, 1 \mathrm{H}), 1.60$ (ddd, $J=25.6,13.2,4.1 \mathrm{~Hz}, 1 \mathrm{H}$ ), 1.23 (ddd, $J=25.6,13.0,4.1 \mathrm{~Hz}, 1 \mathrm{H}$ ) ppm.

${ }^{13} \mathrm{C}-\mathrm{NMR}\left(125 \mathrm{MHz}, \mathrm{CD}_{2} \mathrm{Cl}_{2}\right) \delta=176.5,150.7,146.1,139.2,137.6,131.3,129.2,128.8,127.1$, $126.5,120.4,116.2,92.5,60.0,43.8,35.4,34.9,33.5,29.6$ ppm.

MS (EI) m/z (\%): 480 (88), 263 (100), 217 (10), 159 (10), 144 (31), 117 (10).

HRMS (ESI+) $m / z$ calculated for $\mathrm{C}_{25} \mathrm{H}_{25} \mathrm{~N}_{2} \mathrm{INa}\left(\mathrm{M}+\mathrm{Na}^{+}\right)$503.095463, found 503.095951 .

Mp: $135-136^{\circ} \mathrm{C}(\mathrm{EtOH})$.

1-(4-lodobenzyl)-2-(4-phenylcyclohexylidene)-1-(p-tolyl)hydrazine (1c)<smiles>Cc1ccc(N(Cc2ccc(I)cc2)N=C2CCC(c3ccccc3)CC2)cc1</smiles>

$1 c$

Colorless solid $206 \mathrm{mg}(0.416 \mathrm{mmol}, 83 \%)$.

${ }^{1}$ H-NMR (400 MHz, $\left.\mathrm{CD}_{2} \mathrm{Cl}_{2}\right) \delta=7.67(\mathrm{~d}, J=8.2 \mathrm{~Hz}, 2 \mathrm{H}), 7.31$ (dd, $J=7.6,7.4 \mathrm{~Hz}, 2 \mathrm{H}), 7.19(\mathrm{t}, J=7.4 \mathrm{~Hz}, 1 \mathrm{H}), 7.17-7.14$ $(\mathrm{m}, 4 \mathrm{H}), 7.06(\mathrm{~d}, J=8.2 \mathrm{~Hz}, 2 \mathrm{H}), 6.80(\mathrm{~d}, J=8.5 \mathrm{~Hz}, 2 \mathrm{H}), 4.55$ $(\mathrm{d}, J=14.6 \mathrm{~Hz}, 1 \mathrm{H}), 4.40(\mathrm{~d}, J=14.6 \mathrm{~Hz}, 1 \mathrm{H}), 3.17-3.13(\mathrm{~m}$, $1 \mathrm{H}), 2.77-2.71(\mathrm{~m}, 1 \mathrm{H}), 2.71-2.65(\mathrm{~m}, 1 \mathrm{H}), 2.38(\mathrm{ddd}, J=13.7,13.6,5.0 \mathrm{~Hz}, 1 \mathrm{H}), 2.27(\mathrm{~s}, 3 \mathrm{H})$, 2.08-2.04 (m, $1 \mathrm{H}), 1.82-1.78(\mathrm{~m}, 1 \mathrm{H}), 1.70$ (ddd, $J=13.8,13.5,5.4 \mathrm{~Hz}, 1 \mathrm{H}), 1.58$ (ddd, $J=25.7$, $13.1,4.0 \mathrm{~Hz}, 1 \mathrm{H}), 1.11$ (ddd, $J=25.6,12.9,4.1 \mathrm{~Hz}, 1 \mathrm{H}$ ) ppm.

${ }^{13} \mathrm{C}-\mathrm{NMR}\left(100 \mathrm{MHz}, \mathrm{CD}_{2} \mathrm{Cl}_{2}\right) \delta=175.7,148.9,146.2,139.5,137.6,131.4,130.2,129.7,128.8$, $127.1,126.5,116.8,92.5,60.7,43.9,35.4,35.0,33.6,29.7,20.6$ ppm.

MS (EI) m/z (\%): 494 (100), 477 (23), 373 (22), 277 (97), 217 (40), 144 (41).

HRMS $(E S I+) m / z$ calculated for $\mathrm{C}_{26} \mathrm{H}_{27} \mathrm{~N}_{2} \mathrm{INa}\left(\mathrm{M}+\mathrm{Na}^{+}\right)$517.111115, found 517.110718. 
1-(4-iodobenzyl)-1-(4-methoxyphenyl)-2-(4-phenylcyclohexylidene)hydrazine (1d)<smiles>COc1ccc(N(Cc2ccc(I)cc2)N=C2CCC(c3ccccc3)CC2)cc1</smiles>

$1 d$

Colorless solid $184 \mathrm{mg}(0.36 \mathrm{mmol}, 72 \%)$.

${ }^{1} \mathrm{H}-\mathrm{NMR}\left(500 \mathrm{MHz}, \mathrm{CDCl}_{3}\right) \delta=7.65(\mathrm{~d}, J=8.0 \mathrm{~Hz}, 2 \mathrm{H})$, 7.31$7.28(m, 2 H), 7.19-7.17(m, 1 H), 7.12-7.09(m, 4 H), 6.89-6.88$ (m, 2H), 6.83-6.80 (m, 2H), $4.45(\mathrm{~d}, J=14.1 \mathrm{~Hz}, 1 \mathrm{H}), 4.36(\mathrm{~d}$, $J=14.1 \mathrm{~Hz}, 1 \mathrm{H}), 3.76(\mathrm{~s}, 3 \mathrm{H}), 3.23-3.20(\mathrm{~m}, 1 \mathrm{H}), 2.72-2.63$ (m, 2H), $2.32(\mathrm{ddd}, J=13.5,13.5,4.8 \mathrm{~Hz}, 1 \mathrm{H}), 2.05-2.03(\mathrm{~m}, 1 \mathrm{H}), 1.80-1.78(\mathrm{~m}, 1 \mathrm{H}), 1.67-1.60$ (m, 1H), 1.54 (ddd, $J=25.8,13.2,4.1 \mathrm{~Hz}, 1 \mathrm{H}$ ) ppm.

${ }^{13} \mathrm{C}-\mathrm{NMR}\left(125 \mathrm{MHz}, \mathrm{CDCl}_{3}\right) \delta=174.9,154.7,145.6,145.4,138.9,137.4,131.3,128.7,126.9$, $126.4,119.3,114.4,92.5,61.9,55.7,43.7,35.3,34.7,33.2,29.5$ ppm.

MS (EI) m/z (\%): 493 (100), 389 (95), 262 (15), 217 (15), 172 (45).

HRMS (ESI+): $m / z$ calculated for $\mathrm{C}_{26} \mathrm{H}_{27} \mathrm{~N}_{2} \mathrm{OINa}\left(\mathrm{M}+\mathrm{Na}^{+}\right)$533.106032, found 533.106085.

1-(4-bromophenyl)-1-(4-iodobenzyl)-2-(4-phenylcyclohexylidene)hydrazine (1e)<smiles>Brc1ccc(CN(N=C2CCC(c3ccccc3)CC2)c2ccc(I)cc2)cc1</smiles>

$1 e$
The reaction was conducted on a $0.30 \mathrm{mmol}$ scale. Colorless solid $107 \mathrm{mg}(0.19 \mathrm{mmol}, 64 \%)$.

${ }^{1} \mathrm{H}-\mathrm{NMR}\left(500 \mathrm{MHz}, \mathrm{CDCl}_{3}\right) \delta=7.66-7.64(\mathrm{~m}, 2 \mathrm{H}), 7.32-7.48$ $(m, 4 H), 7.20-7.17(m, 1 H), 7.10-7.07(m, 4 H), 6.74-6.70(m$, $2 \mathrm{H}), 4.54(\mathrm{~d}, J=14.7 \mathrm{~Hz}, 1 \mathrm{H}), 4.40(\mathrm{~d}, J=14.7 \mathrm{~Hz}, 1 \mathrm{H}), 3.10-$ $3.06(\mathrm{~m}, 1 \mathrm{H}), 2.74-2.68(\mathrm{~m}, 2 \mathrm{H}), 2.36(\mathrm{ddd}, J=13.7,13.7,5.0 \mathrm{~Hz}, 1 \mathrm{H}), 2.10-2.05(\mathrm{~m}, 1 \mathrm{H}), 1.84-$ $1.79(\mathrm{~m}, 1 \mathrm{H}), 1.68(\mathrm{ddd}, J=13.7,13.7,5.5 \mathrm{~Hz}, 1 \mathrm{H}), 1.60-1.51(\mathrm{~m}, 1 \mathrm{H}), 1.07$ (ddd, $J=25.8$, 13.3, $4.2 \mathrm{~Hz}, 1 \mathrm{H}) \mathrm{ppm}$.

${ }^{13}$ C-NMR $\left(125 \mathrm{MHz}, \mathrm{CDCl}_{3}\right) \delta=177.4,149.4,145.3,138.0,137.6,131.9,131.0,128.7,126.8$, $126.5,117.6,112.7,92.8,59.9,43.6,35.2,34.7,33.3,29.6$ ppm.

MS (EI) m/z (\%): 560 (100), 558 (100), 343 (70), 341 (70), 262 (15), 217 (30), 172 (20), 170 (20), 144 (35), 117 (20), 93 (30), 91 (30).

HRMS (EI): $m / z$ calculated for $\mathrm{C}_{25} \mathrm{H}_{24} \mathrm{Brl} 558.016774$, found 558.016667 . 
<smiles>Cc1cc(C)cc(N(Cc2ccc(I)cc2)N=C2CCC(c3ccccc3)CC2)c1</smiles>

1f

Colorless solid $113 \mathrm{mg}(0.222 \mathrm{mmol}, 44 \%)$.

${ }^{1} \mathrm{H}-\mathrm{NMR}\left(500 \mathrm{MHz} \mathrm{CDCl}_{3}\right) \delta=7.69-7.67(\mathrm{~m}, 2 \mathrm{H}), 7.34-7.31$ $(\mathrm{m}, 2 \mathrm{H}), 7.23-7.20(\mathrm{~m}, 1 \mathrm{H}), 7.15-7.12(\mathrm{~m}, 4 \mathrm{H}), 6.57-6.53(\mathrm{~m}$, $3 \mathrm{H}), 4.60(\mathrm{~d}, J=14.6 \mathrm{~Hz}, 1 \mathrm{H}), 4.44(\mathrm{~d}, J=14.6 \mathrm{~Hz}, 1 \mathrm{H}), 3.17-$ $3.14(\mathrm{~m}, 1 \mathrm{H}), 2.76-2.70(\mathrm{~m}, 2 \mathrm{H}), 2.39(\mathrm{td}, J=13.7,5.0 \mathrm{~Hz}$, $1 \mathrm{H}), 2.29(\mathrm{~s}, 6 \mathrm{H}), 2.10-2.06(\mathrm{~m}, 1 \mathrm{H}), 1.83-1.78(\mathrm{~m}, 1 \mathrm{H}), 1.68$ $(\mathrm{dt}, J=13.7,5.4 \mathrm{~Hz}, 1 \mathrm{H}), 1.62-1.53(\mathrm{~m}, 1 \mathrm{H}), 1.12-1.03(\mathrm{~m}$,

1H) ppm.

${ }^{13}$ C-NMR $\left(125 \mathrm{MHz}, \mathrm{CDCl}_{3}\right) \delta=176.3,150.7,145.5,138.9,138.6,137.3,131.0,128.6,126.7$, $126.3,122.5,114.0,92.4,60.5,43.6,35.2,34.7,33.1,29.5,21.7$ ppm.

MS (EI) m/z (\%): 508 (90), 291 (100), 217 (10), 144 (27).

HRMS $(\mathrm{ESI}+) \mathrm{m} / z$ calculated for $\mathrm{C}_{27} \mathrm{H}_{29} \mathrm{~N}_{2} \mathrm{INa}\left(\mathrm{M}+\mathrm{Na}^{+}\right)$531.126763, found 531.126824.

1-(4-lodobenzyl)-2-(4-phenylcyclohexylidene)-1-(m-tolyl)hydrazine (1g)<smiles>Cc1cccc(N(Cc2ccc(I)cc2)N=C2CCC(c3ccccc3)CC2)c1</smiles>

19

The reaction was conducted on a $0.3 \mathrm{mmol}$ scale.

Pale yellow solid $73.0 \mathrm{mg}(0.147 \mathrm{mmol}, 49 \%)$. Purified by column chromatography on $\mathrm{SiO}_{2}$ eluting with hexane:EtOAc (9:1).

${ }^{1} \mathrm{H}-\mathrm{NMR}\left(400 \mathrm{MHz}, \mathrm{CDCl}_{3}\right) \delta=7.72-7.68(\mathrm{~m}, 2 \mathrm{H}), 7.36-7.33$ $(\mathrm{m}, 2 \mathrm{H}), 7.25-7.21(\mathrm{~m}, 1 \mathrm{H}), 7.19-7.14(\mathrm{~m}, 5 \mathrm{H}), 6.76-6.70(\mathrm{~m}$, $3 \mathrm{H}), 4.63(\mathrm{~d}, J=14.6 \mathrm{~Hz}, 1 \mathrm{H}), 4.48(\mathrm{~d}, J=14.6 \mathrm{~Hz}, 1 \mathrm{H}), 3.21-3.16(\mathrm{~m}, 1 \mathrm{H}), 2.79-2.71(\mathrm{~m}, 2 \mathrm{H})$, 2.42 (ddd, $J=18.6,13.7,5.0 \mathrm{~Hz}, 1 \mathrm{H}), 2.35(\mathrm{~s}, 3 \mathrm{H}), 2.15-2.09(\mathrm{~m}, 1 \mathrm{H}), 1.87-1.80(\mathrm{~m}, 1 \mathrm{H}), 1.75-$ $1.56(\mathrm{~m}, 2 \mathrm{H}), 1.18-1.07(\mathrm{~m}, 1 \mathrm{H}) \mathrm{ppm}$.

${ }^{13}$ C-NMR $\left(100 \mathrm{MHz}, \mathrm{CDCl}_{3}\right)$ 176.3, 150.6, 145.4, 138.8, 138.8, 137.3, 135.2, 131.0, 129.8, 128.9, 128.6, 127.7, 126.7, 126.3, 121.4, 116.8, 113.2, 92.4, 60.3, 43.6, 35.1, 34.6, 33.1, 29.5, $21.8 \mathrm{ppm}$.

MS (EI) m/z (\%): 494 (75), 277 (100), 217 (10), 144 (35).

HRMS (ESI+) $m / z$ calculated for $\mathrm{C}_{26} \mathrm{H}_{28} \mathrm{~N}_{2} \mathrm{I}\left(\mathrm{M}^{+}\right)$495.128899, found 495.129166. 
<smiles>Cc1cccc(N(Cc2ccc(I)cc2)N=C2CCC(c3ccc4ccccc4c3)CC2)c1</smiles>

1h
The reaction was conducted on a $0.30 \mathrm{mmol}$ scale. Pale yellow solid $135 \mathrm{mg}$ (0.247 mmol, 82\%). Purified by column chromatography on $\mathrm{SiO}_{2}$ eluting with hexane:EtOAc (9:1).

${ }^{1} \mathrm{H}-\mathrm{NMR}\left(400 \mathrm{MHz}, \mathrm{CD}_{2} \mathrm{Cl}_{2}\right) \delta=7.84-7.80(\mathrm{~m}, 3 \mathrm{H}), 7.71-$ $7.68(\mathrm{~m}, 2 \mathrm{H}), 7.59(\mathrm{~d}, J=0.8 \mathrm{~Hz}, 1 \mathrm{H}), 7.49-7.41(\mathrm{~m}, 2 \mathrm{H}), 7.30(\mathrm{dd}, J=8.5,1.7 \mathrm{~Hz}, 1 \mathrm{H}), 7.18-$ $7.11(\mathrm{~m}, 3 \mathrm{H}), 6.73-6.67(\mathrm{~m}, 3 \mathrm{H}), 4.61(\mathrm{~d}, J=14.8 \mathrm{~Hz}, 1 \mathrm{H}), 4.46(\mathrm{~d}, J=14.8 \mathrm{~Hz}, 1 \mathrm{H}), 3.20-3.14$ (m, 1H), 2.97-2.89 (m, 1H), 2.76-2.71 (m, 1H), 2.45 (ddd, J=13.7, 13.6, 5.0 Hz, 1H), $2.32(\mathrm{~s}$, 3H), 2.19-2.14 (m, 1H), 1.93-1.88 (m, 1H), 1.81-1.65 (m, 2H), 1.25 (ddd, J = 25.5, 13.1, 4.2 Hz, 1H) ppm.

${ }^{13} \mathrm{C}-\mathrm{NMR}\left(100 \mathrm{MHz}, \mathrm{CD}_{2} \mathrm{Cl}_{2}\right) \delta=176.2,150.9,143.6,139.4,139.1,137.6,134.0,132.7,131.3$, $129.1,128.4,128.0,127.8,126.3,126.1,125.6,125.0,121.4,117.1,113.5,92.5,60.3,44.0$, $35.4,34.8,33.5,29.7,21.8$ ppm.

MS (EI) m/z (\%): 544 (87), 327 (100), 194 (21), 167 (21), 91 (25).

HRMS (ESI+) $m / z$ calculated for $\mathrm{C}_{30} \mathrm{H}_{29} \mathrm{~N}_{2} \mathrm{INa}\left(\mathrm{M}+\mathrm{Na}^{+}\right)$567.126763, found 567.127244.

2-(4-(4-(tert-Butyl)phenyl)cyclohexylidene)-1-(4-iodobenzyl)-1-phenylhydrazine (1i)

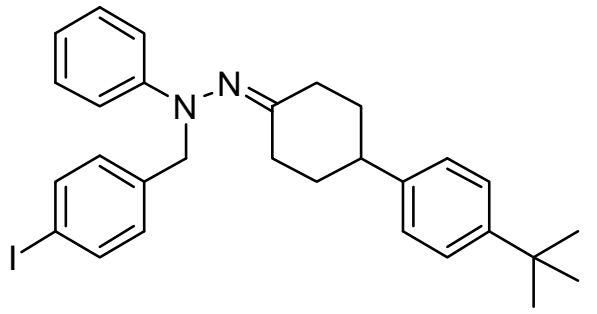

$1 \mathbf{i}$
Colorless solid $222 \mathrm{mg}(0.414 \mathrm{mmol}, 83 \%)$.

${ }^{1} \mathrm{H}-\mathrm{NMR}\left(500 \mathrm{MHz}, \mathrm{CD}_{2} \mathrm{Cl}_{2}\right) \delta=7.67(\mathrm{~d}, J=8.2 \mathrm{~Hz}, 2 \mathrm{H})$, $7.33(\mathrm{~d}, J=8.3 \mathrm{~Hz}, 2 \mathrm{H}), 7.23(\mathrm{dd}, J=8.1,7.8 \mathrm{~Hz}, 2 \mathrm{H}), 7.15$ $(\mathrm{d}, J=8.2 \mathrm{~Hz}, 2 \mathrm{H}), 7.07(\mathrm{~d}, J=8.3 \mathrm{~Hz}, 2 \mathrm{H}), 6.87-6.84(\mathrm{~m}$, $3 \mathrm{H}), 4.60(\mathrm{~d}, J=14.9 \mathrm{~Hz}, 1 \mathrm{H}), 4.45$ (d, $J=14.9 \mathrm{~Hz}, 1 \mathrm{H})$,

3.14-3.08 (m, $1 \mathrm{H}), 2.75-2.65(\mathrm{~m}, 2 \mathrm{H}), 2.42-2.34(\mathrm{~m}, 1 \mathrm{H}), 2.08-2.02(\mathrm{~m}, 1 \mathrm{H}), 1.82-1.77(\mathrm{~m}, 1 \mathrm{H})$, 1.71 (ddd, $J=13.8,13.6,5.5 \mathrm{~Hz}, 1 \mathrm{H}$ ), 1.62-1.53 (m, 1H), 1.30 (s, 9H), 1.11 (ddd, $J=25.6,12.9$, $4.1 \mathrm{~Hz}, 1 \mathrm{H}) \mathrm{ppm}$.

${ }^{13} \mathrm{C}-\mathrm{NMR}\left(125 \mathrm{MHz}, \mathrm{CD}_{2} \mathrm{Cl}_{2}\right) \delta=176.6,150.8,149.4,143.0,139.2,137.6,131.3,129.2,126.7$, $125.7,120.3,116.2,92.5,60.0,43.3,35.4,35.0,34.6,33.6,31.5,29.7$ ppm. 
MS (EI) m/z (\%): 536 (82), 359 (7), 319 (31), 263 (100), 217 (9).

HRMS (ESI+) $m / z$ calculated for $\mathrm{C}_{29} \mathrm{H}_{33} \mathrm{~N}_{2} \mathrm{INa}\left(\mathrm{M}+\mathrm{Na}^{+}\right)$559.158063, found 559.157849.

1-(4-lodobenzyl)-2-(4-(4-methoxyphenyl)cyclohexylidene)-1-phenylhydrazine (1j)<smiles>COc1ccc(C2CCC(=NN(Cc3ccc(I)cc3)c3ccccc3)CC2)cc1</smiles>

$1 \mathrm{j}$

Colorless solid $154 \mathrm{mg}$ (0.302 mmol, 60\%).

${ }^{1} \mathrm{H}-\mathrm{NMR}\left(500 \mathrm{MHz}, \mathrm{CD}_{2} \mathrm{Cl}_{2}\right) \delta=7.67(\mathrm{~d}, J=8.2 \mathrm{~Hz}, 2 \mathrm{H})$, $7.23(\mathrm{dd}, J=8.1,7.8 \mathrm{~Hz}, 2 \mathrm{H}), 7.15(\mathrm{~d}, J=8.2 \mathrm{~Hz}, 2 \mathrm{H})$, $7.05(\mathrm{~d}, J=8.6 \mathrm{~Hz}, 2 \mathrm{H}), 6.87-6.83(\mathrm{~m}, 5 \mathrm{H}), 4.61(\mathrm{~d}, J=$ $14.8 \mathrm{~Hz}, 1 \mathrm{H}), 4.44(\mathrm{~d}, J=14.8 \mathrm{~Hz}, 1 \mathrm{H}), 3.76(\mathrm{~s}, 3 \mathrm{H}), 3.12-$ $3.09(\mathrm{~m}, 1 \mathrm{H}), 2.72-2.66(\mathrm{~m}, 2 \mathrm{H}), 2.38(\mathrm{ddd}, J=13.7,13.6,5.0 \mathrm{~Hz}, 1 \mathrm{H}), 2.05-2.02(\mathrm{~m}, 1 \mathrm{H}), 1.79-$ 1.75 (m 1H), 1.70 (ddd, $J=13.8,13.3,5.4 \mathrm{~Hz}, 1 \mathrm{H}$ ), 1.54 (ddd, $J=25.8,13.1,4.0 \mathrm{~Hz}, 1 \mathrm{H}$ ), 1.06 (ddd, $J=25.5,12.8,4.1 \mathrm{~Hz}, 1 \mathrm{H}$ ) ppm.

${ }^{13} \mathrm{C}-\mathrm{NMR}\left(125 \mathrm{MHz}, \mathrm{CD}_{2} \mathrm{Cl}_{2}\right) \delta=176.7,158.4,150.7,139.2,138.1,137.6,131.3,129.2,127.9$, $120.3,116.2,114.1,92.5,60.0,55.5,43.0,35.4,35.2,33.8,29.7$ ppm.

MS (EI) m/z (\%): 510 (90), 293 (100), 265 (11), 217 (9), 147 (24).

HRMS (ESI+) $m / z$ calculated for $\mathrm{C}_{26} \mathrm{H}_{27} \mathrm{~N}_{2} \mathrm{OINa}\left(\mathrm{M}+\mathrm{Na}^{+}\right)$533.106026, found 533.106125 .

2-(4-(4-Fluorophenyl)cyclohexylidene)-1-(4-iodobenzyl)-1-phenylhydrazine (1k)<smiles>Fc1ccc(C2CCC(=NN(Cc3ccc(I)cc3)c3ccccc3)CC2)cc1</smiles>

$1 \mathrm{k}$
Colorless solid $202 \mathrm{mg}(0.405 \mathrm{mmol}, 81 \%)$

${ }^{1} \mathrm{H}-\mathrm{NMR}\left(500 \mathrm{MHz}, \mathrm{CD}_{2} \mathrm{Cl}_{2}\right) \delta=7.69-7.67(\mathrm{~m}, 2 \mathrm{H})$, 7.26$7.22(\mathrm{~m}, 2 \mathrm{H}), 7.15(\mathrm{~d}, J=8.2 \mathrm{~Hz}, 2 \mathrm{H}), 7.12-7.09(\mathrm{~m}, 2 \mathrm{H})$, 7.03-6.98 (m, 2H), 6.88-6.86 (m, 3H), $4.62(\mathrm{~d}, J=14.7 \mathrm{~Hz}$, $1 \mathrm{H}), 4.43(\mathrm{~d}, J=14.7 \mathrm{~Hz}, 1 \mathrm{H}), 3.15-3.10(\mathrm{~m}, 1 \mathrm{H}), 2.77-2.66$ (m, 2H), 2.39 (ddd, $J=13.7,13.7,5.0 \mathrm{~Hz}, 1 \mathrm{H}), 2.07-2.02(\mathrm{~m}, 1 \mathrm{H}), 1.81-1.75(\mathrm{~m}, 1 \mathrm{H}), 1.70$ (ddd, $J=13.8,13.8,5.5 \mathrm{~Hz}, 1 \mathrm{H}), 1.53(\mathrm{ddd}, J=25.7,13.1,4.1 \mathrm{~Hz}, 1 \mathrm{H}), 1.02(\mathrm{ddd}, J=25.6,12.9,4.1$ $\mathrm{Hz}, 1 \mathrm{H}) \mathrm{ppm}$. 
${ }^{13} \mathrm{C}-\mathrm{NMR}\left(125 \mathrm{MHz}, \mathrm{CD}_{2} \mathrm{Cl}_{2}\right) \delta=176.4,161.7(\mathrm{~d}, J=243.3 \mathrm{~Hz}), 150.7,141.8(\mathrm{~d}, J=3.1 \mathrm{~Hz})$, 139.2, 137.6, 131.4, 129.2, 128.5 (d, $J=7.6 \mathrm{~Hz}$ ), 120.4, 116.2, 115.4 (d, $J=21.1 \mathrm{~Hz}$ ), 92.5, 60.0, 43.1, 35.3, 35.1, 33.7, 29.6 ppm.

MS (EI) m/z (\%): 498 (93), 281 (100), 217 (11), 162 (41), 135 (11).

HRMS (ESI+) $\mathrm{m} / z$ calculated for $\mathrm{C}_{25} \mathrm{H}_{24} \mathrm{~N}_{2} \mathrm{FINa}\left(\mathrm{M}+\mathrm{Na}^{+}\right)$521.086043, found 521.085570.

2-(4-(3,5-Dimethylphenyl)cyclohexylidene)-1-(4-iodobenzyl)-1-phenylhydrazine (1I)<smiles>Cc1cc(C)cc(C2CCC(=NN(Cc3ccc(I)cc3)c3ccccc3)CC2)c1</smiles>

11
Colorless solid $106 \mathrm{mg}$ (0.209 $\mathrm{mmol}, 42 \%)$.

${ }^{1} \mathrm{H}-\mathrm{NMR}\left(500 \mathrm{MHz}, \mathrm{CD}_{2} \mathrm{Cl}_{2}\right) \delta=7.67-7.65(\mathrm{~m}, 2 \mathrm{H})$, 7.25$7.21(\mathrm{~m}, 2 \mathrm{H}), 7.14(\mathrm{~d}, J=8.3 \mathrm{~Hz}, 2 \mathrm{H}), 6.87-6.83(\mathrm{~m}, 4 \mathrm{H})$, $6.76(\mathrm{~s}, 2 \mathrm{H}), 4.59$ (d, J = $15.1 \mathrm{~Hz}, 1 \mathrm{H}), 4.47$ (d, J = $15.1 \mathrm{~Hz}$, $1 \mathrm{H}), 3.14-3.09(\mathrm{~m}, 1 \mathrm{H}), 2.71-2.65(\mathrm{~m}, 2 \mathrm{H}), 2.38$ (ddd, $J=$ 13.7, 13.7, $5.1 \mathrm{~Hz}, 1 \mathrm{H}), 2.29(\mathrm{~s}, 6 \mathrm{H}), 2.07-2.01(\mathrm{~m}, 1 \mathrm{H}), 1.82-1.77(\mathrm{~m}, 1 \mathrm{H}), 1.71$ (ddd, $J=13.9$, 13.6, $5.5 \mathrm{~Hz}, 1 \mathrm{H}), 1.64-1.55(\mathrm{~m}, 1 \mathrm{H}), 1.17(\mathrm{ddd}, J=25.6,12.9,4.1 \mathrm{~Hz}, 1 \mathrm{H}) \mathrm{ppm}$.

${ }^{13} \mathrm{C}-\mathrm{NMR}\left(125 \mathrm{MHz}, \mathrm{CD}_{2} \mathrm{Cl}_{2}\right) \delta=176.4,150.8,145.9,139.2,138.2,137.6,131.1,129.2,128.2$, $124.8,120.3,116.2,92.3,60.1,43.8,35.4,34.8,33.6,29.8,21.5$ ppm.

MS (EI) m/z (\%): 508 (100), 291 (94), 217 (9), 198 (21), 145 (15).

HRMS $(E S I+) m / z$ calculated for $\mathrm{C}_{27} \mathrm{H}_{29} \mathrm{~N}_{2} \mathrm{INa}\left(\mathrm{M}+\mathrm{Na}^{+}\right)$531.126763, found 531.127213.

2-(4-(3,5-Dimethoxyphenyl)cyclohexylidene)-1-(4-iodobenzyl)-1-phenylhydrazine (1 $\mathbf{m})$<smiles>COc1cc(OC)cc(C2CCC(=NN(Cc3ccc(I)cc3)c3ccccc3)CC2)c1</smiles>

Colorless solid $220 \mathrm{mg}$ (0.407 mmol, 81\%).

${ }^{1} \mathrm{H}-\mathrm{NMR}\left(400 \mathrm{MHz}, \mathrm{CDCl}_{3}\right) \delta=7.65(\mathrm{~d}, J=8.2 \mathrm{~Hz}, 2 \mathrm{H})$, 7.26 (dd, J = 7.9, $7.9 \mathrm{~Hz}, 2 \mathrm{H}), 7.12(\mathrm{~d}, J=8.2 \mathrm{~Hz}, 2 \mathrm{H})$, 6.92-9.87 (m, 3H), $6.32(\mathrm{~s}, 3 \mathrm{H}), 4.56(\mathrm{~s}, 2 \mathrm{H}), 3.79(\mathrm{~s}, 6 \mathrm{H})$, 3.18-3.14 $(m, 1 H), 2.78-2.67(m, 2 H), 2.42-2.34(m, 1 H)$, 2.16-2.06 (m, 1H), 1.92-1.82 (m, 1H), 1.74-1.60 (m, 2H), 1.32-1.22 (m, 1H) ppm. 
${ }^{13} \mathrm{C}-\mathrm{NMR}\left(100 \mathrm{MHz}, \mathrm{CDCl}_{3}\right) \delta=161.0,150.6,148.0,138.8,137.5,130.6,129.1,120.7,116.3$, $105.1,98.1,92.4,60.6,55.4,43.9,35.1,34.3,33.2,29.6$ ppm.

MS (EI) m/z (\%): 540 (96), 359 (6), 323 (100), 217 (21), 177 (10).

HRMS (ESI+) $m / z$ calculated for $\mathrm{C}_{27} \mathrm{H}_{29} \mathrm{~N}_{2} \mathrm{O}_{2} \mathrm{INa}\left(\mathrm{M}+\mathrm{Na}^{+}\right)$563.116592, found 563.116729.

2-(4-(3-Chlorophenyl)cyclohexylidene)-1-(4-iodobenzyl)-1-phenylhydrazine (1n)<smiles>Clc1cccc(C2CCC(=NN(Cc3ccc(I)cc3)c3ccccc3)CC2)c1</smiles>

1n

Colorless solid $192 \mathrm{mg}$ (0.373 mmol, 75\%).

${ }^{1} \mathrm{H}-\mathrm{NMR}\left(400 \mathrm{MHz}, \mathrm{CD}_{2} \mathrm{Cl}_{2}\right) \delta=7.72-7.70(\mathrm{~m}, 2 \mathrm{H}), 7.32-$ $7.26(\mathrm{~m}, 3 \mathrm{H}), 7.23-7.17(\mathrm{~m}, 4 \mathrm{H}), 7.08(\mathrm{~d}, J=7.6 \mathrm{~Hz}, 1 \mathrm{H})$, 6.92-6.90 (m, 3H), $4.63(\mathrm{~d}, J=14.9 \mathrm{~Hz}, 1 \mathrm{H}), 4.51(\mathrm{~d}, J=$ $14.9 \mathrm{~Hz}, 1 \mathrm{H}), 3.19-3.15(\mathrm{~m}, 1 \mathrm{H}), 2.80-2.73(\mathrm{~m}, 2 \mathrm{H}), 2.42$ (ddd, $J=18.6,13.6,5.0 \mathrm{~Hz}, 1 \mathrm{H}), 2.12-2.08(\mathrm{~m}, 1 \mathrm{H}), 1.86-1.70(\mathrm{~m}, 2 \mathrm{H}), 1.62$ (ddd, $J=25.6$, $13.1,4.1 \mathrm{~Hz}, 1 \mathrm{H}$ ), 1.15 (ddd, $J=25.6,13.1,4.1 \mathrm{~Hz}, 1 \mathrm{H}$ ) ppm.

${ }^{13} \mathrm{C}-\mathrm{NMR}\left(100 \mathrm{MHz}, \mathrm{CD}_{2} \mathrm{Cl}_{2}\right) \delta=175.8,150.9,148.2,139.2,137.7,134.4,131.3,130.3,129.3$, $127.4,126.7,125.4,120.6,116.4,92.6,60.3$, 43.6, 35.3, 34.6, 33.4, 29.6 ppm.

MS (EI) m/z (\%): 514/516 (76/28), 297/299 (100/35), 217 (16), 178 (23), 159 (12).

HRMS (ESI+) $m / z$ calculated for $\mathrm{C}_{25} \mathrm{H}_{24} \mathrm{~N}_{2} \mathrm{ClINa}\left(\mathrm{M}+\mathrm{Na}^{+}\right)$537.056494, found 537.056326.

1-(4-lodobenzyl)-2-(4-(naphthalen-2-yl)cyclohexylidene)-1-phenylhydrazine (10)<smiles>Ic1ccc(CN(N=C2CCC(c3ccc4ccccc4c3)CC2)c2ccccc2)cc1</smiles>

10
Colorless solid $203 \mathrm{mg}(0.382 \mathrm{mmol}, 76 \%)$

${ }^{1} \mathrm{H}-\mathrm{NMR}\left(400 \mathrm{MHz}, \mathrm{CD}_{2} \mathrm{Cl}_{2}\right) \delta=7.88-7.84(\mathrm{~m}, 3 \mathrm{H}), 7.74-$ $7.72(m, 2 H), 7.64(s, 1 H), 7.52-7.45(m, 2 H), 7.35-7.27$ $(\mathrm{m}, 3 \mathrm{H}), 7.21(\mathrm{~d}, J=8.3 \mathrm{~Hz}, 2 \mathrm{H}), 6.94-6.90(\mathrm{~m}, 3 \mathrm{H}), 4.67$ (d, J=14.9 Hz, 1H), 4.52 (d, J=14.9 Hz, 1H), 3.25-3.19 ( $m, 1 \mathrm{H}), 3.00-2.93(\mathrm{~m}, 1 \mathrm{H}), 2.81-2.76(\mathrm{~m}, 1 \mathrm{H}), 2.49(\mathrm{ddd}, J=18.8,13.7,5.1 \mathrm{~Hz}, 1 \mathrm{H}), 2.25-2.18$ (m, $1 \mathrm{H}), 1.99-1.93(\mathrm{~m}, 1 \mathrm{H}), 1.86-1.70(\mathrm{~m}, 2 \mathrm{H}), 1.30$ (ddd, $J=25.5,13.2,4.3 \mathrm{~Hz}, 1 \mathrm{H}) \mathrm{ppm}$. 
${ }^{13}$ C-NMR $\left(100 \mathrm{MHz}, \mathrm{CD}_{2} \mathrm{Cl}_{2}\right) \delta=176.4,150.9,143.6,139.3,137.7,134.0,132.7,131.3,129.3$, $128.4,128.0,127.9,126.3,126.2,125.7,125.0,120.5,116.3,92.5,60.2,44.0,35.4,34.8$, 33.5, 29.7 ppm.

MS (EI) m/z (\%): 530 (100), 313 (83), 217 (17), 194 (12), 167 (28), 77 (38).

HRMS $(E S I+) m / z$ calculated for $\mathrm{C}_{29} \mathrm{H}_{27} \mathrm{~N}_{2} \mathrm{INa}\left(\mathrm{M}+\mathrm{Na}^{+}\right)$553.111113, found 553.110976.

1-(4-iodobenzyl)-2-(4-methylcyclohexylidene)-1-phenylhydrazine (1p)<smiles>CC1CCC(=NN(Cc2ccc(I)cc2)c2ccccc2)CC1</smiles>

$1 p$

Colorless solid $72.0 \mathrm{mg}(0.17 \mathrm{mmol}, 34 \%)$.

${ }^{1} \mathrm{H}-\mathrm{NMR}\left(400 \mathrm{MHz}, \mathrm{CDCl}_{3}\right) \delta=7.62-7.59(\mathrm{~m}, 2 \mathrm{H}), 7.24-7.18(\mathrm{~m}$, $2 \mathrm{H}), 7.08-7.06(\mathrm{~m}, 2 \mathrm{H}), 6.87-6.78(\mathrm{~m}, 3 \mathrm{H}), 4.52(\mathrm{~d}, J=15.2 \mathrm{~Hz}, 1 \mathrm{H})$, $4.47(\mathrm{~d}, J=15.1 \mathrm{~Hz}, 1 \mathrm{H}), 2.95-2.90(\mathrm{~m}, 1 \mathrm{H}), 2.59-2.53(\mathrm{~m}, 1 \mathrm{H}), 2.22$ (ddd, $J=13.2,13.2,5.1 \mathrm{~Hz}, 1 \mathrm{H}), 1.90-1.83(\mathrm{~m}, 1 \mathrm{H}), 1.64-1.55(\mathrm{~m}, 3 \mathrm{H}), 1.18-1.07(\mathrm{~m}, 1 \mathrm{H}), 0.88$ (d, $J=6.5 \mathrm{~Hz}, 3 \mathrm{H}), 0.76-0.66(\mathrm{~m}, 1 \mathrm{H}) \mathrm{ppm}$.

${ }^{13} \mathrm{C}-\mathrm{NMR}\left(100 \mathrm{MHz}, \mathrm{CDCl}_{3}\right) \delta=177.2,150.5,138.9,137.4,130.6,129.1,120.3,116.0,92.3$, $60.3,35.4,34.7,34.2,31.9,29.2,21.5$ ppm.

MS (EI) m/z (\%): 418 (50), 217 (10), 201 (100), 77 (15).

HRMS $(E S I+) m / z$ calculated for $\mathrm{C}_{20} \mathrm{H}_{23} \mathrm{~N}_{2} \mathrm{INa}\left(\mathrm{M}+\mathrm{Na}^{+}\right) 441.079813$, found 411.079454 .

2-([1,1'-Bi(cyclohexan)]-4-ylidene)-1-(4-iodobenzyl)-1-phenylhydrazine (1q)

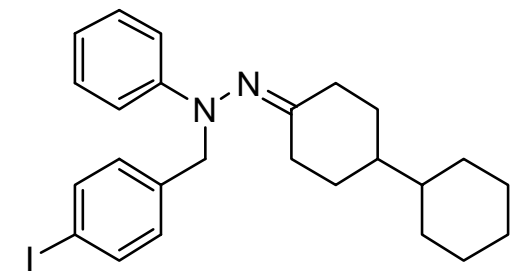

$1 q$

Colorless solid $67.6 \mathrm{mg}(0.139 \mathrm{mmol}, 28 \%)$.

${ }^{1} \mathrm{H}-\mathrm{NMR}\left(400 \mathrm{MHz}, \mathrm{CD}_{2} \mathrm{Cl}_{2}\right) \delta=7.65-7.61(\mathrm{~m}, 2 \mathrm{H})$, 7.23-7.18 $(\mathrm{m}, 2 \mathrm{H}), 7.10(\mathrm{~d}, J=8.3 \mathrm{~Hz}, 2 \mathrm{H}), 6.85-6.79(\mathrm{~m}, 3 \mathrm{H}), 4.55(\mathrm{~d}, J=$ $15.0 \mathrm{~Hz}, 1 \mathrm{H}), 4.42(\mathrm{~d}, J=15.0 \mathrm{~Hz}, 1 \mathrm{H}), 2.97-2.92(\mathrm{~m}, 1 \mathrm{H}), 2.59$ $2.53(\mathrm{~m}, 1 \mathrm{H}), 2.17(\mathrm{td}, J=6.8,5.0 \mathrm{~Hz}, 1 \mathrm{H}), 1.92-1.87(\mathrm{~m}, 1 \mathrm{H})$, 1.74-1.71 (m, 2H), 1.65-1.61 (m, 3H), 1.58-1.53 (m, 2H), 1.33-1.07 (m, 6H), 0.98-0.89 (m, 2H), 0.72 (ddd, $J=23.9,12.2,4.1 \mathrm{~Hz}, 1 \mathrm{H}$ ) ppm. 
${ }^{13} \mathrm{C}-\mathrm{NMR}\left(100 \mathrm{MHz}, \mathrm{CD}_{2} \mathrm{Cl}_{2}\right) \delta=177.8,150.8,139.3,137.6,131.1,129.2,120.1,116.0,92.4$, $59.9,54.3,53.3,42.8,42.7,35.2,30.7,30.6,29.6,29.4,27.2,27.1$ ppm.

MS (EI) $m / z$ (\%): 486 (87), 469 (9), 269 (100), 217 (13), 176 (7).

HRMS $(E S I+) m / z$ calculated for $\mathrm{C}_{25} \mathrm{H}_{31} \mathrm{~N}_{2} \mathrm{INa}\left(\mathrm{M}+\mathrm{Na}^{+}\right)$509.142412, found 509.142420.

Mp: $78.2-78.6^{\circ} \mathrm{C}(\mathrm{EtOH})$.

2-(4-(tert-Butyl)cyclohexylidene)-1-(4-iodobenzyl)-1-phenylhydrazine (1r)<smiles>CC(C)(C)C1CCC(=NN(Cc2ccc(I)cc2)c2ccccc2)CC1</smiles>

$1 \mathrm{r}$

Colorless solid $113 \mathrm{mg}$ (0.246 mmol, 49\%).

${ }^{1}$ H-NMR (400 MHz, $\left.\mathrm{CDCl}_{3}\right) \delta=7.66-7.62(\mathrm{~m}, 2 \mathrm{H}), 7.26-7.22(\mathrm{~m}$, $2 \mathrm{H}), 7.11(\mathrm{~d}, J=8.3 \mathrm{~Hz}, 2 \mathrm{H}), 6.89-6.83(\mathrm{~m}, 3 \mathrm{H}), 4.57(\mathrm{~d}, J=14.9$ $\mathrm{Hz}, 1 \mathrm{H}), 4.47(\mathrm{~d}, J=14.9 \mathrm{~Hz}, 1 \mathrm{H}), 3.09-3.03(\mathrm{~m}, 1 \mathrm{H}), 2.67-2.61$ $(\mathrm{m}, 1 \mathrm{H}), 2.20(\mathrm{td}, J=13.4,4.8 \mathrm{~Hz}, 1 \mathrm{H}), 2.01-1.96(\mathrm{~m}, 1 \mathrm{H}), 1.74-$ $1.69(\mathrm{~m}, 1 \mathrm{H}), 1.51$ (ddd, $J=19.4,13.9,5.5 \mathrm{~Hz}, 1 \mathrm{H}), 1.25-1.09(\mathrm{~m}$, $2 \mathrm{H}), 0.84(\mathrm{~s}, 9 \mathrm{H}), 0.76-0.65(\mathrm{~m}, 1 \mathrm{H}) \mathrm{ppm}$.

${ }^{13} \mathrm{C}-\mathrm{NMR}\left(100 \mathrm{MHz}, \mathrm{CDCl}_{3}\right) \delta=177.2,150.1,138.3,136.9,133.3,128.6,119.8,115.5,91.9$, 59.6, 47.0, 34.7, 32.0, 29.2, 27.7, 27.2, 26.5 ppm.

MS (EI) m/z (\%): 460 (47), 243 (100), 217 (13), 187 (5).

HRMS (ESI+) $m / z$ calculated for $\mathrm{C}_{23} \mathrm{H}_{29} \mathrm{~N}_{2} \mathrm{INa}\left(\mathrm{M}+\mathrm{Na}^{+}\right)$483.126767, found 483.126607. 
4-(2-(4-iodobenzyl)-2-phenylhydrazono)cyclohexyl benzoate (1s)<smiles>O=C(OC1CCC(=NN(Cc2ccc(I)cc2)c2ccccc2)CC1)c1ccccc1</smiles>

1s

The reaction was conducted on a $0.25 \mathrm{mmol}$ scale.

Colorless solid $70.6 \mathrm{mg}$ (0.135 mmol, 54\%).

${ }^{1} \mathrm{H}-\mathrm{NMR}\left(500 \mathrm{MHz}, \mathrm{CDCl}_{3}\right) \delta=8.01-8.00(\mathrm{~m}, 2 \mathrm{H}), 7.63(\mathrm{~d}, J=$ 8.4 Hz, 2H), 7.56-7.53 (m, $1 \mathrm{H}), 7.43(\mathrm{dd}, J=7.8,7.8 \mathrm{~Hz}, 2 \mathrm{H})$, 7.25-7.22 (m, 2H), $7.09(\mathrm{~d}, J=8.2 \mathrm{~Hz}, 2 \mathrm{H}), 6.90-6.84(\mathrm{~m}, 3 \mathrm{H}), 5.22-5.18(\mathrm{~m}, 1 \mathrm{H}), 4.52(\mathrm{~s}, 2 \mathrm{H})$, 2.70-2.64 (m, 1H), 2.52-2.45 (m, 2H), 2.40-2.34 (m, $1 \mathrm{H}), 2.05-1.93(\mathrm{~m}, 2 \mathrm{H}), 1.70-1.60(\mathrm{~m}, 2 \mathrm{H})$, ${ }^{13} \mathrm{C}-\mathrm{NMR}\left(125 \mathrm{MHz}, \mathrm{CDCl}_{3}\right) \delta=174.5,165.9,150.6,138.7,137.5,133.2,130.7,130.5,129.7$, $129.2,128.5,120.9,116.4,92.4,70.3,60.7,31.3,31.2,30.1,25.8$ ppm.

MS (EI) $m / z$ (\%): 524 (70), 217 (12), 185 (100).

HRMS (ESI+): $m / z$ calculated for $\mathrm{C}_{26} \mathrm{H}_{25} \mathrm{~N}_{2} \mathrm{O}_{2} \mathrm{INa}\left(\mathrm{M}+\mathrm{Na}^{+}\right)$547.085296, found 547.085746.

2-(4-(2-(4-lodobenzyl)-2-phenylhydrazono)cyclohexyl)isoindoline-1,3-dione (1t)<smiles>O=C1c2ccccc2C(=O)N1C1CCC(=NN(Cc2ccc(I)cc2)c2ccccc2)CC1</smiles>

1t
Colorless solid $217 \mathrm{mg}$ (0.395 mmol, 79\%).

${ }^{1} \mathrm{H}-\mathrm{NMR}\left(500 \mathrm{MHz}, \mathrm{CD}_{2} \mathrm{Cl}_{2}\right) \delta=7.81-7.71(\mathrm{~m}, 4 \mathrm{H}), 7.66(\mathrm{~d}$, $J=8.0 \mathrm{~Hz}, 2 \mathrm{H}), 7.26-7.23(\mathrm{~m}, 2 \mathrm{H}), 7.12(\mathrm{~d}, J=7.9 \mathrm{~Hz}, 2 \mathrm{H})$, 6.90-6.84 (m, 3H), $4.58(\mathrm{~d}, J=15.6 \mathrm{~Hz}, 1 \mathrm{H}), 4.54(\mathrm{~d}, J=$ $15.6 \mathrm{~Hz}, 1 \mathrm{H}), 4.39-4.34(\mathrm{~m}, 1 \mathrm{H}), 3.16(\mathrm{~d}, J=14.9 \mathrm{~Hz}, 1 \mathrm{H})$, $2.75(\mathrm{~d}, J=13.0 \mathrm{~Hz}, 1 \mathrm{H}), 2.54-2.45(\mathrm{~m}, 1 \mathrm{H}), 2.43-2.37(\mathrm{~m}$,

$1 \mathrm{H}), 2.17-2.08(\mathrm{~m}, 1 \mathrm{H}), 1.99-1.98(\mathrm{~m}, 1 \mathrm{H}), 1.76-1.69(\mathrm{~m}, 2 \mathrm{H}) \mathrm{ppm}$.

${ }^{13} \mathrm{C}-\mathrm{NMR}\left(125 \mathrm{MHz}, \mathrm{CDCl}_{3}\right) \delta=173.4,168.3,150.8,139.1,137.7,134.3,132.3,130.6,129.2$, $123.3,120.6,116.5,92.5,60.6,49.6,34.2,29.7,28.5,28.5$ ppm.

MS (EI) m/z (\%): 549 (100), 385 (6), 332 (62), 217 (18), 185 (67), 168 (15).

HRMS (ESI+) $m / z$ calculated for $\mathrm{C}_{27} \mathrm{H}_{24} \mathrm{~N}_{3} \mathrm{O}_{2} \mathrm{INa}\left(\mathrm{M}+\mathrm{Na}^{+}\right) 572.080541$, found 572.080076 . Mp: $150-152{ }^{\circ} \mathrm{C}(\mathrm{EtOH})$. 
1-(4-iodobenzyl)-2-(4-methyl-4-phenylcyclohexylidene)-1-phenylhydrazine (1u)

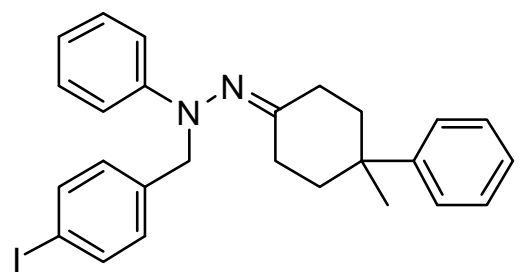

Colorless solid $208 \mathrm{mg}$ (0.421 mmol, 84\%)

${ }^{1} \mathrm{H}-\mathrm{NMR}\left(500 \mathrm{MHz}, \mathrm{CD}_{2} \mathrm{Cl}_{2}\right) \delta=7.64(\mathrm{~d}, J=8.2 \mathrm{~Hz}, 2 \mathrm{H})$ ) 7.37-

$7.33(\mathrm{~m}, 4 \mathrm{H}), 7.24-7.17(\mathrm{~m}, 3 \mathrm{H}), 7.11(\mathrm{~d}, J=8.1 \mathrm{~Hz}, 2 \mathrm{H}), 6.83$ (t, $J=7.3 \mathrm{~Hz}, 1 \mathrm{H}), 6.79(\mathrm{~d}, J=8.1 \mathrm{~Hz}, 2 \mathrm{H}), 4.53(\mathrm{~d}, J=15.2 \mathrm{~Hz}$,

$1 \mathrm{H}), 4.47(\mathrm{~d}, J=15.2 \mathrm{~Hz}, 1 \mathrm{H}), 2.60-2.55(\mathrm{~m}, 1 \mathrm{H}), 2.52-2.48(\mathrm{~m}, 1 \mathrm{H}), 2.36-2.29(\mathrm{~m}, 2 \mathrm{H}), 2.69-$ $1.98(\mathrm{~m}, 2 \mathrm{H}), 1.77-1.71(\mathrm{~m}, 1 \mathrm{H}), 1.40-1.34(\mathrm{~m}, 1 \mathrm{H}), 1.21$ (s, 3H) ppm.

${ }^{13} \mathrm{C}-\mathrm{NMR}\left(125 \mathrm{MHz}, \mathrm{CD}_{2} \mathrm{Cl}_{2}\right) \delta=176.5,150.7,147.4,139.2,137.6,130.9,129.2,128.9,126.1$, $126.1,120.2,116.1,92.3,60.0,38.3,38.2,36.8,31.8,30.6,26.6$ ppm.

MS (EI) m/z (\%): 494 (100), 277 (90), 249 (21), 217 (11), 171 (21), 131 (11), 77 (23).

HRMS (El) $m / z$ calculated for $\mathrm{C}_{26} \mathrm{H}_{27} \mathrm{~N}_{2} \mathrm{l}$ 494.121893, found 494.121489.

2-((cis)-3,4-Diphenylcyclopentylidene)-1-(4-iodobenzyl)-1-phenylhydrazine (1v)

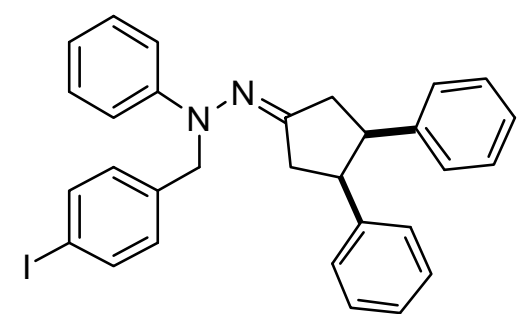

1v

Pale yellow solid $215 \mathrm{mg}$ (0.397 mmol, 79\%).

Purified by column chromatography on $\mathrm{SiO}_{2}$ eluting with hexane:EtOAc (4:1).

${ }^{1} \mathrm{H}-\mathrm{NMR}\left(500 \mathrm{MHz}, \mathrm{CD}_{2} \mathrm{Cl}_{2}\right) \delta=7.71(\mathrm{~d}, J=8.3 \mathrm{~Hz}, 2 \mathrm{H}), 7.30$ (dd, $J=8.4,7.5 \mathrm{~Hz}, 2 \mathrm{H}), 7.25(\mathrm{~d}, J=8.2 \mathrm{~Hz}, 2 \mathrm{H}), 7.13-7.01(\mathrm{~m}$, $8 \mathrm{H}), 6.96(\mathrm{t}, J=7.3 \mathrm{~Hz}, 1 \mathrm{H}), 6.66(\mathrm{~d}, J=6.9 \mathrm{~Hz}, 2 \mathrm{H}), 6.57(\mathrm{~d}, J=6.9 \mathrm{~Hz}, 2 \mathrm{H}), 4.68(\mathrm{~d}, J=15.1$ $\mathrm{Hz}, 2 \mathrm{H}), 4.61(\mathrm{~d}, J=15.1 \mathrm{~Hz}, 2 \mathrm{H}), 3.65-3.60(\mathrm{~m}, 1 \mathrm{H}), 3.59-3.54(\mathrm{~m}, 1 \mathrm{H}), 2.98-2.97(\mathrm{~m}, 2 \mathrm{H})$, $2.68(\mathrm{dd}, J=18.7,6.8 \mathrm{~Hz}, 1 \mathrm{H}), 2.59(\mathrm{dd}, J=18.7,7.9 \mathrm{~Hz}, 1 \mathrm{H})$.

${ }^{13}$ C-NMR $\left(125 \mathrm{MHz}, \mathrm{CD}_{2} \mathrm{Cl}_{2}\right) \delta=179.6,151.1,140.6,140.5,139.7,137.7,130.8,129.4,128.4$, $128.0,128.0,126.5,121.6,118.1,92.6,62.3,49.0,48.4,38.0,36.8$ ppm MS (EI) $m / z$ (\%): 542 (100), 325 (53), 221 (66).

HRMS $(E S I+) m / z$ calculated for $\mathrm{C}_{30} \mathrm{H}_{27} \mathrm{~N}_{2} \mathrm{INa}\left(\mathrm{M}+\mathrm{Na}^{+}\right)$565.111116, found 565.110673. 


\section{Tetrahydrocarbazoles}

General procedure:

A reaction vial was charged with catalyst $\mathbf{5 f}(3.33 \mathrm{mg}, 0.005 \mathrm{mmol})$, Amberlite ${ }^{\circledR}$ CG50 (200 $\mathrm{mg})$, the corresponding hydrazone $1 \mathrm{a}-1 \mathbf{q}(0.100 \mathrm{mmol})$ and molecular sieves $4 \AA$ (50 mg). Under an atmosphere of argon, benzene $(1.0 \mathrm{~mL})$ was added and the resulting mixture was stirred in the sealed vial at $30^{\circ} \mathrm{C}$ until complete conversion was indicated by TLC. The crude reaction mixture was directly submitted to column chromatography on $\mathrm{SiO}_{2}$.

The racemates were prepared by heating a 1:1 mixture of the corresponding hydrazone and diphenyl phosphate to $80^{\circ} \mathrm{C}$ in toluene for $1 \mathrm{~h}$. The crude reaction mixture was directly submitted to column chromatography on $\mathrm{SiO}_{2}$.

(S)-9-Benzyl-3-phenyl-2,3,4,9-tetrahydro-1H-carbazole (2a)

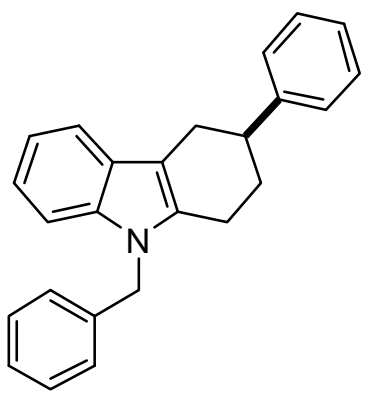

$2 a$

Purification: Hexane:EtOAc (9:1). Colorless solid $31.8 \mathrm{mg}$ (0.094 $\mathrm{mmol}, 94 \%)$.

${ }^{1} \mathrm{H}-\mathrm{NMR}\left(500 \mathrm{MHz}, \mathrm{CDCl}_{3}\right) \delta=7.43(\mathrm{~d}, J=7.2 \mathrm{~Hz}, 1 \mathrm{H}), 7.29-7.25(\mathrm{~m}$, $4 \mathrm{H}), 7.22-7.14(\mathrm{~m}, 5 \mathrm{H}), 7.08-7.04(\mathrm{~m}, 1 \mathrm{H}), 7.04-7.01(\mathrm{~m}, 1 \mathrm{H}), 6.95(\mathrm{~d}, J$ $=7.1 \mathrm{~Hz}, 2 \mathrm{H}), 5.23(\mathrm{~d}, J=16.9 \mathrm{~Hz}, 1 \mathrm{H}), 5.19(\mathrm{~d}, J=16.9 \mathrm{~Hz}, 1 \mathrm{H})$, 3.09$3.05(\mathrm{~m}, 1 \mathrm{H}), 3.04-2.98(\mathrm{~m}, 1 \mathrm{H}), 2.84-2.79(\mathrm{~m}, 1 \mathrm{H}), 2.73-2.68(\mathrm{~m}, 2 \mathrm{H})$, 2.18-2.12 (m, 1H), 2.08-1.98 (m, 1H) ppm.

${ }^{13}$ C-NMR $\left(125 \mathrm{MHz}, \mathrm{CDCl}_{3}\right) \delta=146.8,138.3,137.0,135.3,128.9,128.6,127.4,127.3,127.2$, $126.4,126.3,121.1,119.2,117.9,109.9,109.2,46.5,41.1,30.5,29.5,22.5$ ppm.

MS (EI) $m / z$ (\%): 337 (70), 233 (100), 218 (14), 142 (5), 115 (7), 91 (34).

HRMS (EI) $m / z$ calculated for $\mathrm{C}_{25} \mathrm{H}_{23} \mathrm{~N} 337.183049$, found 337.183072.

The enantiomeric ratio was determined by reversed phase HPLC analysis using Daicel Chiralcel OJ-RH column: $\mathrm{MeCN}: \mathrm{H}_{2} \mathrm{O}=90: 10$, flow rate $1.0 \mathrm{~mL} / \mathrm{min}, \lambda=220 \mathrm{~nm}: \tau_{1}=5.10 \mathrm{~min}$, $\tau_{2}=7.87 \mathrm{~min}$. 


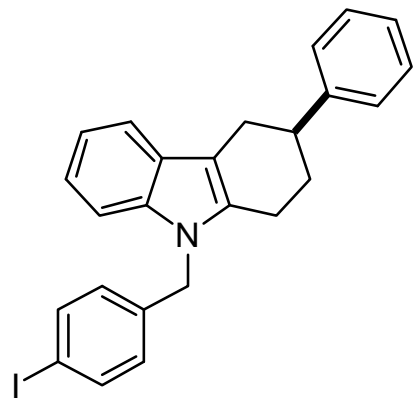

$2 b$

Purification: Hexane:EtOAc (9:1). Colorless solid $45.8 \mathrm{mg}(0.099$ $\mathrm{mmol}, 99 \%)$.

${ }^{1} \mathbf{H}-\mathbf{N M R}\left(500 \mathrm{MHz}, \mathrm{CD}_{2} \mathrm{Cl}_{2}\right) \delta=7.62-7.60(\mathrm{~m}, 2 \mathrm{H}), 7.48(\mathrm{~d}, J=7.3$ $\mathrm{Hz}, 1 \mathrm{H}), 7.35-7.34(\mathrm{~m}, 4 \mathrm{H}), 7.26-7.22(\mathrm{~m}, 1 \mathrm{H}), 7.19(\mathrm{~d}, J=7.8 \mathrm{~Hz}$, 1H), 7.10 (ddd, $J=8.0,7.0,1.2 \mathrm{~Hz}, 1 \mathrm{H}$ ), 7.07 (ddd, $J=7.5,7.2,1.1$ $\mathrm{Hz}, 1 \mathrm{H}), 6.76(\mathrm{~d}, J=8.4 \mathrm{~Hz}, 2 \mathrm{H}), 5.25$ (d, J=17.1 Hz, 1H), 5.20 (d, $J$ $=17.1 \mathrm{~Hz}, 1 \mathrm{H}), 3.13-3.05(\mathrm{~m}, 2 \mathrm{H}), 2.89-2.83(\mathrm{~m}, 1 \mathrm{H}), 2.78-2.75(\mathrm{~m}$, $2 \mathrm{H}), 2.26-2.20(\mathrm{~m}, 1 \mathrm{H}), 2.16-2.08(\mathrm{~m}, 1 \mathrm{H}) \mathrm{ppm}$.

${ }^{13} \mathrm{C}-\mathrm{NMR}\left(125 \mathrm{MHz}, \mathrm{CD}_{2} \mathrm{Cl}_{2}\right) \delta=147.1,138.6,138.1,137.1,135.5,128.7,128.6,127.7,127.4$, 126.5, 121.2, 119.4, 118.2, 110.3, 109.2, 92.7, 46.2, 41.3, 30.7, 29.6, 22.6 ppm.

MS (EI) m/z (\%): 463 (100), 359 (97), 344 (5), 232 (16), 217 (37).

HRMS $(E S I+) m / z$ calculated for $\mathrm{C}_{25} \mathrm{H}_{22} \mathrm{NINa}\left(\mathrm{M}+\mathrm{Na}^{+}\right) 486.068919$, found 486.068606 .

Mp: $123-125^{\circ} \mathrm{C}\left(\mathrm{CH}_{2} \mathrm{Cl}_{2}\right)$.

$[\alpha]_{D}^{25}:-4.10^{\circ}, \mathrm{c}=0.34\left(\mathrm{CHCl}_{3}, \mathrm{er}=95: 5\right)$.

The enantiomeric ratio was determined by reversed phase HPLC analysis using Daicel Chiralcel OJ-RH column: $\mathrm{MeCN}: \mathrm{H}_{2} \mathrm{O}=90: 10$, flow rate $1.0 \mathrm{~mL} / \mathrm{min}, \lambda=220 \mathrm{~nm}: \tau_{1}=11.07$ $\min , \tau_{2}=13.23 \min$.

(S)-9-(4-lodobenzyl)-6-methyl-3-phenyl-2,3,4,9-tetrahydro-1H-carbazole (2c)

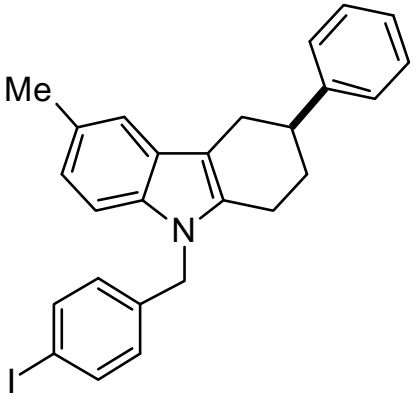

2c

Purification: Hexane:EtOAc (15:1). Colorless solid $47.2 \mathrm{mg}(0.099$ $\mathrm{mmol}, 99 \%)$.

${ }^{1} \mathrm{H}-\mathrm{NMR}\left(400 \mathrm{MHz}, \mathrm{CD}_{2} \mathrm{Cl}_{2}\right) \delta=7.50 .7 .47(\mathrm{~m}, 2 \mathrm{H}), 7.23-7.22(\mathrm{~m}$, 2H), $7.16(\mathrm{~s}, 1 \mathrm{H}), 7.14-7.11(\mathrm{~m}, 1 \mathrm{H}), 6.95(\mathrm{~d}, J=8.2 \mathrm{~Hz}, 1 \mathrm{H}), 6.82$ (dd, $J=8.2,1.4 \mathrm{~Hz}, 1 \mathrm{H}), 6.64-6.62(\mathrm{~m}, 2 \mathrm{H}), 5.09(\mathrm{~d}, J=17.0 \mathrm{~Hz}, 1 \mathrm{H})$, $5.04(\mathrm{~d}, J=17.0 \mathrm{~Hz}, 1 \mathrm{H}), 3.00,2.92(\mathrm{~m}, 2 \mathrm{H}), 2.75-2.68(\mathrm{~m}, 1 \mathrm{H})$,

2.64-2.61 (m, 2H), $2.31(\mathrm{~s}, 3 \mathrm{H}), 2.11-2.07(\mathrm{~m}, 1 \mathrm{H}), 2.03-1.95(\mathrm{~m}, 1 \mathrm{H}) \mathrm{ppm}$. 
${ }^{13} \mathrm{C}-\mathrm{NMR}\left(100 \mathrm{MHz}, \mathrm{CDCl}_{3}\right) \delta=147.2,138.8,138.1,135.6,135.5,128.8,128.7,128.6,128.0$, $127.4,126.5,122.8,118.1,109.9,109.0,92.6,46.3,41.3,30.8,29.6,22.6,21.6$ ppm.

MS (EI) m/z (\%): 477 (100), 373 (96), 246 (18), 217 (15), 156 (20).

HRMS (ESI+) $m / z$ calculated for $\mathrm{C}_{26} \mathrm{H}_{24} \mathrm{NINa}\left(\mathrm{M}+\mathrm{Na}^{+}\right)$500.084567, found 500.084895 .

The enantiomeric ratio was determined by reversed phase HPLC analysis using Daicel Chiralcel OD-RH column: $\mathrm{MeCN}: \mathrm{H}_{2} \mathrm{O}=80: 20$, flow rate $1.0 \mathrm{~mL} / \mathrm{min}, \lambda=220 \mathrm{~nm}: \tau_{1}=13.76$ $\min , \tau_{2}=14.86 \min$.

(S)-9-(4-iodobenzyl)-6-methoxy-3-phenyl-2,3,4,9-tetrahydro-1H-carbazole (2d)

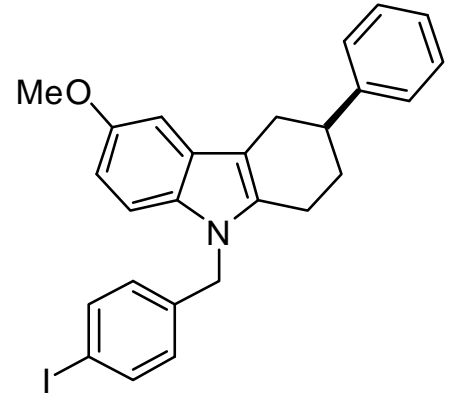

2d

Purification: Hexane:EtOAc (9:1). Colorless solid $47.1 \mathrm{mg}(0.096$ $\mathrm{mmol}, 96 \%)$.

${ }^{1} \mathrm{H}-\mathrm{NMR}\left(500 \mathrm{MHz}, \mathrm{CDCl}_{3}\right) \delta=7.49(\mathrm{~d}, 2 \mathrm{H}, J=8.2 \mathrm{~Hz}), 7.25-7.23$ $(\mathrm{m}, 4 \mathrm{H}), 7.17-7.14(\mathrm{~m}, 1 \mathrm{H}), 7.26-7.22(\mathrm{~m}, 1 \mathrm{H}), 6.97(\mathrm{~d}, J=8.8 \mathrm{~Hz}$, $1 \mathrm{H}), 6.87(\mathrm{~d}, J=2.2 \mathrm{~Hz}, 1 \mathrm{H}), 6.69(\mathrm{dd}, J=8.8,2.2 \mathrm{~Hz}, 1 \mathrm{H}), 6.63(\mathrm{~d}$, $J=8.1 \mathrm{~Hz}, 2 \mathrm{H}), 5.10(\mathrm{~d}, J=17.0 \mathrm{~Hz}, 1 \mathrm{H}), 5.05(\mathrm{~d}, J=17.0 \mathrm{~Hz}, 1 \mathrm{H})$, $3.75(\mathrm{~s}, 3 \mathrm{H}), 3.02-2.95(\mathrm{~m}, 2 \mathrm{H}), 2.78-2.73(\mathrm{~m}, 1 \mathrm{H}), 2.64-2.62(\mathrm{~m}, 2 \mathrm{H}), 2.136-2.11(\mathrm{~m}, 1 \mathrm{H})$, 2.046-1.99 (m, 1H) ppm.

${ }^{13}$ C-NMR $\left(100 \mathrm{MHz}, \mathrm{CDCl}_{3}\right) \delta=154.1,146.6,138.1,137.9,135.8,132.0,128.6,128.2,127.6$, $127.1,126.4,110.8,109.9,109.7,100.4,92.6,56.0,46.1,41.1,30.4,29.5,22.5$ ppm. MS (EI) m/z (\%): 493 (100), 389 (95), 262 (15), 172 (45).

HRMS (ESI) $\mathrm{m} / z$ calculated for $\mathrm{C}_{26} \mathrm{H}_{24} \mathrm{NOINa}\left(\mathrm{M}+\mathrm{Na}^{+}\right)$516.079478, found 516.079951 .

The enantiomeric ratio was determined by reversed phase HPLC analysis using Daicel Chiralcel OJ-RH column: $\mathrm{MeCN}: \mathrm{H}_{2} \mathrm{O}=90: 10$, flow rate $1.0 \mathrm{~mL} / \mathrm{min}, \lambda=220 \mathrm{~nm}: \tau_{1}=8.73 \mathrm{~min}$, $\tau_{2}=10.12 \mathrm{~min}$. 
(S)-6-bromo-9-(4-iodobenzyl)-3-phenyl-2,3,4,9-tetrahydro-1H-carbazole (2e)<smiles>Brc1ccc2c(c1)c1c(n2Cc2ccc(I)cc2)CCC(c2ccccc2)C1</smiles>

$2 e$

Purification: Hexane:EtOAc (9:1). Colorless solid $44.2 \mathrm{mg}(0.075$ $\mathrm{mmol}, 75 \%)$.

${ }^{1}$ H-NMR $\left(500 \mathrm{MHz}, \mathrm{CDCl}_{3}\right) \delta=7.58-7.57(\mathrm{~m}, 3 \mathrm{H}), 7.34-7.27(\mathrm{~m}$, $4 \mathrm{H}), 7.24-7.22(\mathrm{~m}, 1 \mathrm{H}), 7.17(\mathrm{dd}, J=8.6,1.4 \mathrm{~Hz}, 1 \mathrm{H}), 7.02$ (d, $J=8.6$ $\mathrm{Hz}, 1 \mathrm{H}), 6.68(\mathrm{~d}, J=8.0 \mathrm{~Hz}, 2 \mathrm{H}), 5.18(\mathrm{~d}, J=17.2 \mathrm{~Hz}, 1 \mathrm{H}), 5.14(\mathrm{~d}, J$ $=17.2 \mathrm{~Hz}, 1 \mathrm{H}), 3.06-3.02(\mathrm{~m}, 2 \mathrm{H}), 2.83-2.78(\mathrm{~m}, 1 \mathrm{H}), 2.70-2.69(\mathrm{~m}$, $2 \mathrm{H}), 2.21-2.19(\mathrm{~m}, 1 \mathrm{H}), 2.11-2.05(\mathrm{~m}, 1 \mathrm{H}) \mathrm{ppm}$.

${ }^{13}$ C-NMR $\left(100 \mathrm{MHz}, \mathrm{CDCl}_{3}\right) \delta=146.2,138.0,137.5,136.5,135.5,129.1,128.6,128.1,127.1$, $126.5,123.9,120.8,112.6,110.5,110.0,92.9,46.1$, 40.8, 30.3, 29.1, 22.3 ppm.

MS (EI) m/z (\%): 543 (100), 541 (100), 437 (95), 435 (95), 358 (10), 310 (15), 231 (25), 217 (70), $167(10,141$ (15), 90 (30).

HRMS (EI) $m / z$ calculated for $\mathrm{C}_{25} \mathrm{H}_{21} \mathrm{NBrl} 540.990224$, found 540.989881 .

The enantiomeric ratio was determined by reversed phase HPLC analysis using Daicel Chiralcel OJ-RH column: $\mathrm{MeCN}: \mathrm{H}_{2} \mathrm{O}=90: 10$, flow rate $1.0 \mathrm{~mL} / \mathrm{min}, \lambda=220 \mathrm{~nm}: \tau_{1}=11.36$ $\min , \tau_{2}=14.02 \min$.

(S)-9-(4-lodobenzyl)-5,7-dimethyl-3-phenyl-2,3,4,9-tetrahydro-1H-carbazole (2f)

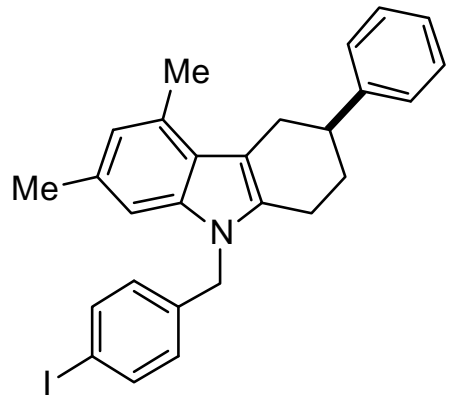

$2 f$

Purification: Hexane:EtOAc (9:1). Colorless solid $44.1 \mathrm{mg}(0.090$ $\mathrm{mmol}, 90 \%)$.

${ }^{1} \mathrm{H}-\mathrm{NMR}\left(400 \mathrm{MHz}, \mathrm{CD}_{2} \mathrm{Cl}_{2}\right) \delta=7.51-7.48(\mathrm{~m}, 2 \mathrm{H}), 7.22(\mathrm{~d}, J=1.4$ $\mathrm{Hz}, 2 \mathrm{H}), 7.14-7.11(\mathrm{~m}, 1 \mathrm{H}), 6.69(\mathrm{~s}, 1 \mathrm{H}), 6.65-6.62(\mathrm{~m}, 2 \mathrm{H}), 6.52$ (s, 1H), 5.07 (d, J = $17.1 \mathrm{~Hz}, 1 \mathrm{H}), 5.02(\mathrm{~d}, J=17.1 \mathrm{~Hz}, 1 \mathrm{H}), 3.32-$ $3.24(\mathrm{~m}, 1 \mathrm{H}), 3.00-2.90(\mathrm{~m}, 2 \mathrm{H}), 2.63-2.59(\mathrm{~m}, 2 \mathrm{H}), 2.48(\mathrm{~s}, 3 \mathrm{H})$, $2.24(\mathrm{~s}, 3 \mathrm{H}), 2.09-2.02(\mathrm{~m}, 1 \mathrm{H}), 2.00-1.92(\mathrm{~m}, 1 \mathrm{H}) \mathrm{ppm}$.

${ }^{13} \mathrm{C}-\mathrm{NMR}\left(100 \mathrm{MHz}, \mathrm{CD}_{2} \mathrm{Cl}_{2}\right) \delta=147.4,138.9,138.1,137.6,134.1,131.0,130.3,128.8,128.6$, $127.5,126.5,124.5,122.6,110.7,107.1,92.6,46.1,32.5,22.7,21.7,20.0$ ppm. 
MS (EI) m/z (\%): 460 (47), 243 (100), 217 (13), 187 (5).

HRMS (ESI+) $\mathrm{m} / z$ calculated for $\mathrm{C}_{27} \mathrm{H}_{26} \mathrm{NINa}\left(\mathrm{M}+\mathrm{Na}^{+}\right)$514.100212, found 514.100342.

The enantiomeric ratio was determined by reversed phase HPLC analysis using Daicel Chiralcel OJ-RH column: $\mathrm{MeCN}: \mathrm{H}_{2} \mathrm{O}=90: 10$, flow rate $1.0 \mathrm{~mL} / \mathrm{min}, \lambda=220 \mathrm{~nm}: \tau_{1}=14.84$ $\min , \tau_{2}=19.55 \min$.

(S)-9-(4-lodobenzyl)-7-methyl-3-phenyl-2,3,4,9-tetrahydro-1H-carbazole (2g)

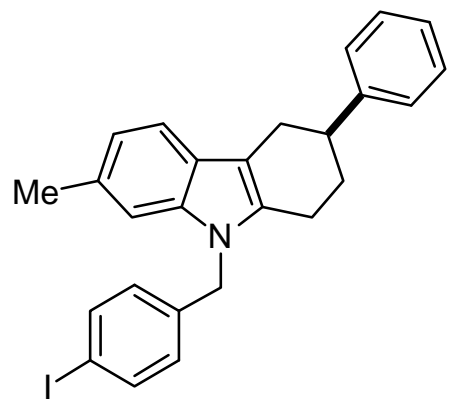

$2 g$

Purification: Hexane:EtOAc (9:1). Colorless solid $43.5 \mathrm{mg}(0.091$ mmol, 91\%).

The product was obtained as a 6:1 mixture with its regioisomer 9-(4-iodobenzyl)-5-methyl-3-phenyl-2,3,4,9-tetrahydro-1Hcarbazole (determined by ${ }^{1} \mathrm{H}$ NMR).

${ }^{1} \mathrm{H}-\mathrm{NMR}\left(400 \mathrm{MHz}, \mathrm{CD}_{2} \mathrm{Cl}_{2}\right) \delta=7.63-7.60(\mathrm{~m}, 2 \mathrm{H}), 7.38-7.31(\mathrm{~m}$, 5H), 7.27-7.22 (m, 1H), 7.00 (s, 1H), 6.93-6.90 (m, 1H), 6.78-6.74 (m, 2H), $5.21(\mathrm{~d}, J=17.2 \mathrm{~Hz}$, $1 \mathrm{H}), 5.16(\mathrm{~d}, J=17.2 \mathrm{~Hz}, 1 \mathrm{H}), 3.11-3.03(\mathrm{~m}, 2 \mathrm{H}), 2.88-2.80(\mathrm{~m}, 1 \mathrm{H}), 2.77-2.72(\mathrm{~m}, 2 \mathrm{H}), 2.42(\mathrm{~s}$, $3 \mathrm{H}), 2.23-2.15(\mathrm{~m}, 1 \mathrm{H}), 2.14-2.05(\mathrm{~m}, 1 \mathrm{H}) \mathrm{ppm}$.

${ }^{13} \mathrm{C}-\mathrm{NMR}\left(100 \mathrm{MHz}, \mathrm{CD}_{2} \mathrm{Cl}_{2}\right) \delta=147.2,138.8,138.1,137.5,134.8,131.1,128.7,128.6,127.4$, 126.5, 125.6, 121.0, 117.9, 110.1, 109.3, 92.6, 46.1, 41.3, 30.8, 29.7, 22.6, 22.0 ppm.

MS (EI) m/z (\%): 477 (100), 373 (75), 246 (15), 217 (20).

HRMS (ESI+) $m / z$ calculated for $\mathrm{C}_{26} \mathrm{H}_{24} \mathrm{NINa}\left(\mathrm{M}+\mathrm{Na}^{+}\right) 500.084567$, found 500.084895 .

The enantiomeric ratio was determined by reversed phase HPLC analysis using Daicel Chiralcel OJ-RH column: $\mathrm{MeCN}: \mathrm{H}_{2} \mathrm{O}=80: 20$, flow rate $1.0 \mathrm{~mL} / \mathrm{min}, \lambda=220 \mathrm{~nm}: \tau_{1}=25.05$ $\min , \tau_{2}=30.18 \mathrm{~min}$. 
(S)-9-(4-lodobenzyl)-7-methyl-3-(naphthalen-2-yl)-2,3,4,9-tetrahydro-1H-carbazole (2h)

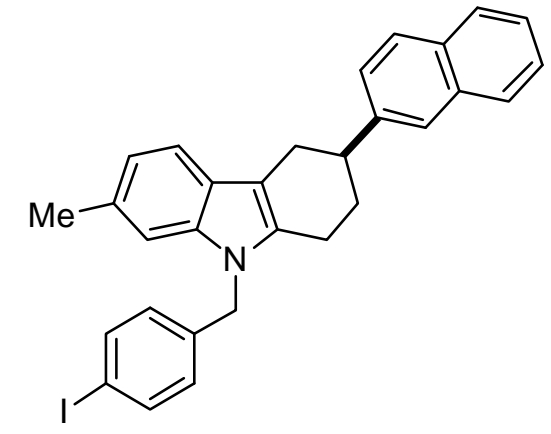

$2 \mathrm{~h}$

Purification: Hexane:EtOAc (9:1). Colorless solid $47.7 \mathrm{mg}$ (0.090 mmol, 90\%).

The product was obtained as a 6:1 mixture with its regioisomer 9-(4-iodobenzyl)-5-methyl-3-(naphthalen-2-yl)2,3,4,9-tetrahydro-1H-carbazole (determined by ${ }^{1} \mathrm{H}$ NMR). ${ }^{1} \mathrm{H}-\mathrm{NMR}\left(400 \mathrm{MHz}, \mathrm{CD}_{2} \mathrm{Cl}_{2}\right) \delta=7.85-7.80(\mathrm{~m}, 3 \mathrm{H}), 7.77$ (d, J $=0.5 \mathrm{~Hz}, 1 \mathrm{H}), 7.62-7.59(\mathrm{~m}, 2 \mathrm{H}), 7.53-7.43(\mathrm{~m}, 3 \mathrm{H}), 7.38(\mathrm{~d}, J=7.9 \mathrm{~Hz}, 1 \mathrm{H}), 7.01(\mathrm{~s}, 1 \mathrm{H}), 6.93-$ $6.91(\mathrm{~m}, 1 \mathrm{H}), 6.78-6.75(\mathrm{~m}, 2 \mathrm{H}), 5.22(\mathrm{~d}, J=7.9 \mathrm{~Hz}, 1 \mathrm{H}), 5.18(\mathrm{~d}, J=7.9 \mathrm{~Hz}, 1 \mathrm{H}), 3.28-3.21(\mathrm{~m}$, $1 \mathrm{H}), 3.17(\mathrm{dd}, J=15.3,5.0 \mathrm{~Hz}, 1 \mathrm{H}), 2.99-2.92(\mathrm{~m}, 1 \mathrm{H}), 2.83-2.76(\mathrm{~m}, 2 \mathrm{H}), 2.43(\mathrm{~s}, 3 \mathrm{H}), 2.33-$ $2.27(\mathrm{~m}, 1 \mathrm{H}), 2.24-2.15(\mathrm{~m}, 1 \mathrm{H}) \mathrm{ppm}$.

${ }^{13}$ C-NMR $\left(100 \mathrm{MHz}, \mathrm{CD}_{2} \mathrm{Cl}_{2}\right) \delta=144.6,138.8,138.1,137.5,134.8,134.1,132.7,131.1,130.7$, $128.5,128.2$, 127.9, 127.9, 126.5, 126.2, 125.7, 125.6, 125.3, 121.1, 117.9, 109.3, 92.6, 46.1, $41.4,30.8,29.5,22.6,21.9 \mathrm{ppm}$.

MS (EI) m/z (\%): 527 (96), 373 (100), 358 (7), 246 (14), 217 (20), 156 (10).

HRMS (ESI+) $\mathrm{m} / \mathrm{z}$ calculated for $\mathrm{C}_{30} \mathrm{H}_{26} \mathrm{NINa}\left(\mathrm{M}+\mathrm{Na}^{+}\right)$550.100216, found 550.100582.

The enantiomeric ratio was determined by HPLC analysis using Daicel Chiralpak AD-3 column: $n$-heptane: $i$-PrOH $=99: 1$, flow rate $1.0 \mathrm{~mL} / \mathrm{min}, \lambda=220 \mathrm{~nm}: \tau_{1}=10.43 \mathrm{~min}, \tau_{2}=$ $11.49 \mathrm{~min}$.

(S)-3-(4-(tert-Butyl)phenyl)-9-(4-iodobenzyl)-2,3,4,9-tetrahydro-1H-carbazole (2i)

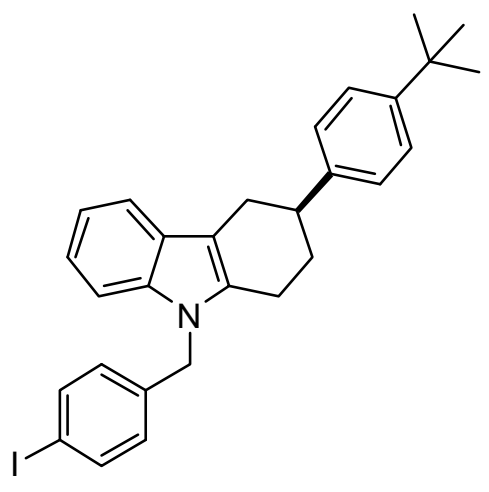

2i

Purification: Hexane:EtOAc (9:1). Colorless solid $49.6 \mathrm{mg}$ (0.095 mmol, 95\%).

${ }^{1}$ H-NMR $\left(500 \mathrm{MHz}, \mathrm{CD}_{2} \mathrm{Cl}_{2}\right) \delta=7.62-7.60(\mathrm{~m}, 2 \mathrm{H}), 7.48(\mathrm{~d}, J=$ $7.3 \mathrm{~Hz}, 1 \mathrm{H}), 7.38-7.36(\mathrm{~m}, 2 \mathrm{H}), 7.28-7.27(\mathrm{~m}, 2 \mathrm{H}), 7.19(\mathrm{~d}, J=$ $7.8 \mathrm{~Hz}, 1 \mathrm{H}$ ), 7.10 (ddd, $J=8.0,7.0,1.0 \mathrm{~Hz}, 1 \mathrm{H}$ ), 7.06 (ddd, $J=$ 7.5, 7.1, $1.0 \mathrm{~Hz}, 1 \mathrm{H}), 6.76(\mathrm{~d}, J=8.3 \mathrm{~Hz}, 2 \mathrm{H}), 5.24(\mathrm{~d}, J=17.1$ $\mathrm{Hz}, 1 \mathrm{H}), 5.20(\mathrm{~d}, J=17.0 \mathrm{~Hz}, 1 \mathrm{H}), 3.11-3.03(\mathrm{~m}, 2 \mathrm{H}), 2.87-2.82$ 
(m, 1H), 2.77-2.75 (m, 2H), 2.22-2.19 (m, 1H), 2.14-2.06 (m, 1H), 1.34 (s, 9H) ppm.

${ }^{13} \mathrm{C}-\mathrm{NMR}\left(125 \mathrm{MHz}, \mathrm{CD}_{2} \mathrm{Cl}_{2}\right) \delta=149.4,144.0,138.6,138.1,137.1,135.5,128.6,127.7,127.0$, 125.6, 121.2, 119.4, 118.2, 110.4, 109.2, 92.6, 46.2, 40.7, 34.6, 31.5, 30.8, 29.7, 22.6 ppm.

MS (EI) m/z (\%): 519 (84), 359 (100), 232 (12), 217 (26).

HRMS (ESI+) $m / z$ calculated for $\mathrm{C}_{29} \mathrm{H}_{30} \mathrm{NINa}\left(\mathrm{M}+\mathrm{Na}^{+}\right) 542.131518$, found 542.131503.

Mp: $170-171^{\circ} \mathrm{C}(\mathrm{MeOH})$.

The enantiomeric ratio was determined by reversed phase HPLC analysis using Daicel Chiralcel OJ-RH column: $\mathrm{MeCN}: \mathrm{H}_{2} \mathrm{O}=90: 10$, flow rate $1.0 \mathrm{~mL} / \mathrm{min}, \lambda=220 \mathrm{~nm}: \tau_{1}=8.52 \mathrm{~min}$, $\tau_{2}=9.58 \mathrm{~min}$.

(S)-9-(4-lodobenzyl)-3-(4-methoxyphenyl)-2,3,4,9-tetrahydro-1H-carbazole (2j)

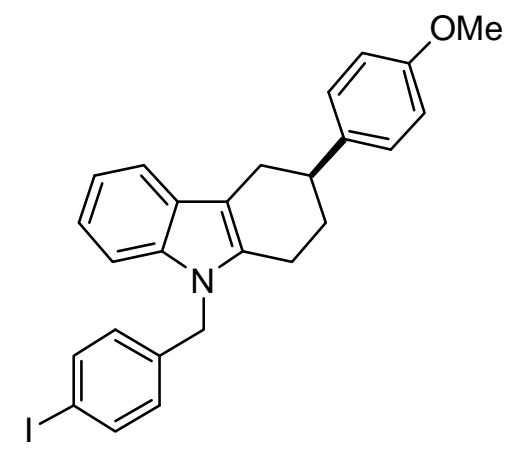

2j

Purification: Hexane:EtOAc (9:1). Colorless solid $46.2 \mathrm{mg}$ (0.094 mmol, 94\%).

${ }^{1} \mathrm{H}-\mathrm{NMR}\left(500 \mathrm{MHz}, \mathrm{CD}_{2} \mathrm{Cl}_{2}\right) \delta=7.61(\mathrm{~d}, J=8.3 \mathrm{~Hz}, 2 \mathrm{H}), 7.49$ (d, $7.3 \mathrm{~Hz}, 1 \mathrm{H}), 7.26(\mathrm{~d}, J=8.6 \mathrm{~Hz}, 2 \mathrm{H}), 7.19(\mathrm{~d}, J=7.9 \mathrm{~Hz}, 1 \mathrm{H})$, 7.11 (dd, $J=7.6,6.3 \mathrm{~Hz}, 1 \mathrm{H}), 7.07$ (dd, $J=7.0,6.7 \mathrm{~Hz}, 1 \mathrm{H})$, $6.88(\mathrm{~d}, J=8.6 \mathrm{~Hz}, 2 \mathrm{H}), 6.76(\mathrm{~d}, J=8.3 \mathrm{~Hz}, 2 \mathrm{H}), 5.23(\mathrm{~d}, J=$ $17.1 \mathrm{~Hz}, 1 \mathrm{H}), 5.19$ (d, J = $17.1 \mathrm{~Hz}, 1 \mathrm{H}), 3.80(\mathrm{~s}, 3 \mathrm{H}), 3.10-3.01$ (m, 2H), 2.84-2.79 (m, 1H), 2.77-2.73 (m, 2H), 2.20-2.17 (m, 1H), 2.11-2.04 (m, 1H) ppm.

${ }^{13} \mathrm{C}-\mathrm{NMR}\left(125 \mathrm{MHz}, \mathrm{CD}_{2} \mathrm{Cl}_{2}\right) \delta=158.4,139.1,138.6,138.0,137.1,135.5,128.6,128.2,127.7$, $121.2,119.3,118.2,114.0,110.4,109.2$, 92.7, 55.5, 46.2, 40.4, 30.9, 29.8, 22.6 ppm.

MS (EI) $m / z$ (\%): 493 (72), 359 (100), 344 (5), 232 (14), 217 (31).

HRMS (ESI+) $m / z$ calculated for $\mathrm{C}_{26} \mathrm{H}_{24} \mathrm{NOINa}\left(\mathrm{M}+\mathrm{Na}^{+}\right)$516.079477, found 516.079157.

Mp: $148-150{ }^{\circ} \mathrm{C}\left(\mathrm{CH}_{2} \mathrm{Cl}_{2}\right)$.

The enantiomeric ratio was determined by HPLC analysis using Daicel Chiralcel OD-3 column: $n$-heptane:i-PrOH = 99.5:0.5, flow rate $1.0 \mathrm{~mL} / \mathrm{min}, \lambda=220 \mathrm{~nm}: \tau_{1}=41.52 \mathrm{~min}, \tau_{2}=47.12$ min and by reversed phase HPLC analysis using Daicel Chiralcel OD-RH column: $\mathrm{MeCN}: \mathrm{H}_{2} \mathrm{O}=$ 65:35, flow rate $1.0 \mathrm{~mL} / \mathrm{min}, \lambda=220 \mathrm{~nm}: \tau_{1}=37.18 \mathrm{~min}, \tau_{2}=40.01 \mathrm{~min}$. 


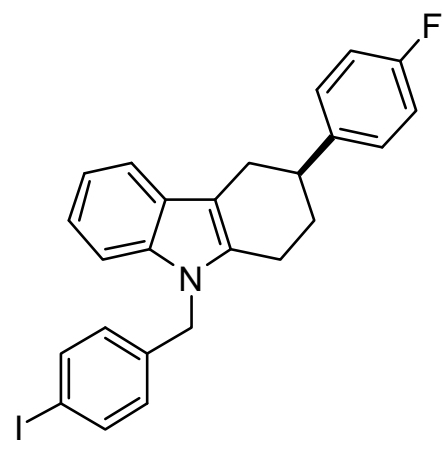

$2 k$

Purification: Hexane:EtOAc (9:1). Colorless solid $47.8 \mathrm{mg}(0.099$ mmol, 99\%).

${ }^{1} \mathrm{H}-\mathrm{NMR}\left(400 \mathrm{MHz}, \mathrm{CD}_{2} \mathrm{Cl}_{2}\right) \delta=7.62-7.60(\mathrm{~m}, 2 \mathrm{H})$, 7.49-7.47 (m, $1 H)$, 7.32-7.29 $(m, 2 H), 7.21-7.18(m, 1 H), 7.13-7.10(m, 1 H)$, 7.08-7.06 (m, 1H), 7.06-7.01 (m, 2H), 6.77-6-75 (m, 2H), 5.24 (d, J $=17.1 \mathrm{~Hz}, 1 \mathrm{H}), 5.20(\mathrm{~d}, J=17.1 \mathrm{~Hz}, 1 \mathrm{H}), 3.13-3.05(\mathrm{~m}, 2 \mathrm{H}), 2.86-$ $2.81(\mathrm{~m}, 1 \mathrm{H}), 2.79-2.74(\mathrm{~m}, 2 \mathrm{H}), 2.23-2.17(\mathrm{~m}, 1 \mathrm{H}), 2.11-2.03(\mathrm{~m}$, 1H) ppm.

${ }^{13} \mathrm{C}-\mathrm{NMR}\left(100 \mathrm{MHz}, \mathrm{CD}_{2} \mathrm{Cl}_{2}\right) \delta=161.7$ (d, $\left.J=242.7 \mathrm{~Hz}\right), 142.8$ (d, $\left.J=3.1 \mathrm{~Hz}\right), 138.6,138.1$, $137.1,135.4,128.8$ (d, $J=7.6 \mathrm{~Hz}), 128.6,127.6,121.3,119.4,118.1,115.3$ (d, J=21.1 Hz), $110.1,109.3,92.7,46.2,40.5,30.8,29.7,22.5$ ppm.

MS (EI) m/z (\%): 481 (100), 359 (95), 344 (5), 232 (17), 217 (39).

HRMS $(\mathrm{ESI}+) \mathrm{m} / z$ calculated for $\mathrm{C}_{25} \mathrm{H}_{21} \mathrm{NFINa}\left(\mathrm{M}+\mathrm{Na}^{+}\right)$504.059492, found 504.059141. Mp: $132-133^{\circ} \mathrm{C}\left(\mathrm{CH}_{2} \mathrm{Cl}_{2}\right)$.

The enantiomeric ratio was determined by HPLC analysis using Daicel Chiralcel OD-3 column: $n$-heptane: $i-\mathrm{PrOH}=98: 2$, flow rate $1.0 \mathrm{~mL} / \mathrm{min}, \lambda=220 \mathrm{~nm}: \tau_{1}=12.67 \mathrm{~min}, \tau_{2}=14.70 \mathrm{~min}$.

(S)-3-(3,5-Dimethylphenyl)-9-(4-iodobenzyl)-2,3,4,9-tetrahydro-1H-carbazole (2I)

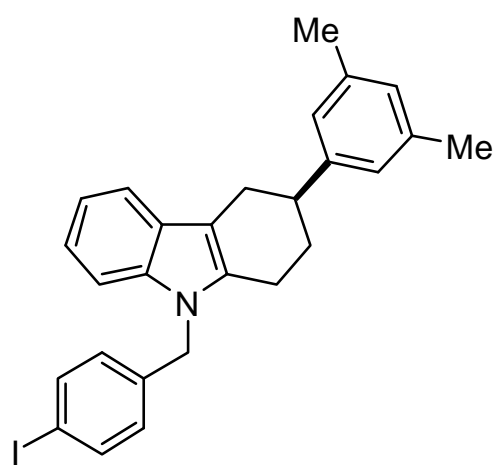

2l

Purification: Hexane:EtOAc (9:1). Colorless solid $43.7 \mathrm{mg}$ (0.089 mmol, 89\%).

${ }^{1} \mathrm{H}-\mathrm{NMR}\left(400 \mathrm{MHz}, \mathrm{CD}_{2} \mathrm{Cl}_{2}\right) \delta=7.63-7.60(\mathrm{~m}, 2 \mathrm{H})$ ) 7.49-7.47 (m, 1H), 7.20-7.18 (m, 1H), 7.10 (ddd, $J=7.9,7.0,1.2 \mathrm{~Hz}, 1 \mathrm{H}$ ), 7.06 (ddd, $J=7.4,7.1,1.2 \mathrm{~Hz}, 1 \mathrm{H}), 6.96(\mathrm{~s}, 2 \mathrm{H}), 6.89$ (s, 1H), 6.79-6.76 (m, 2H), 5.25 (d, $J=17.1 \mathrm{~Hz}, 1 \mathrm{H}), 5.20$ (d, $J=17.1$ $\mathrm{Hz}, 1 \mathrm{H}), 3.07$ (dd, $J=14.8,5.1 \mathrm{~Hz}, 1 \mathrm{H}), 3.02-2.95(\mathrm{~m}, 1 \mathrm{H})$, 2.86-2.81 (m, 1H), 2.79-2.76 (m, 2H), $2.32(\mathrm{~s}, 6 \mathrm{H}), 2.21-2.15(\mathrm{~m}, 1 \mathrm{H}), 2.12-2.05(\mathrm{~m}, 1 \mathrm{H}) \mathrm{ppm}$. 
${ }^{13}$ C-NMR $\left(100 \mathrm{MHz}, \mathrm{CD}_{2} \mathrm{Cl}_{2}\right) \delta=147.0,138.7,138.2,138.1,137.1,135.5,128.6,128.1,127.8$, $125.2,121.2$, 119.3, 118.2, 110.5, 109.2, 92.7, 46.2, 41.3, 30.8, 29.8, 22.8, 21.5 ppm.

MS (EI) m/z (\%): 491 (82), 359 (100), 344 (5), 232 (14), 217 (32).

HRMS (ESI+) $m / z$ calculated for $\mathrm{C}_{27} \mathrm{H}_{26} \mathrm{NINa}\left(\mathrm{M}+\mathrm{Na}^{+}\right)$514.100212, found 514.100059.

The enantiomeric ratio was determined by HPLC analysis using Daicel Chiralcel OD-H column: $n$-heptane: $i-\mathrm{PrOH}=98: 2$, flow rate $0.5 \mathrm{~mL} / \mathrm{min}, \lambda=220 \mathrm{~nm}: \tau_{1}=19.07 \mathrm{~min}, \tau_{2}=21.31 \mathrm{~min}$.

(S)-3-(3,5-Dimethoxyphenyl)-9-(4-iodobenzyl)-2,3,4,9-tetrahydro-1H-carbazole (2m)

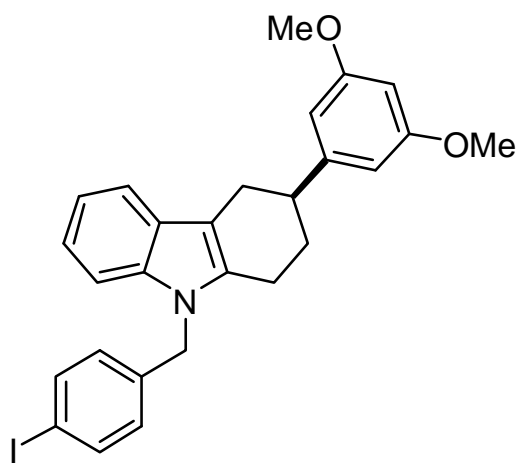

$2 \mathrm{~m}$

Purification: Hexane:EtOAc (6:1). Colorless solid $51.2 \mathrm{mg}$ (0.098 mmol, 98\%).

${ }^{1} \mathbf{H}-\mathrm{NMR}\left(400 \mathrm{MHz}, \mathrm{CD}_{2} \mathrm{Cl}_{2}\right) \delta=7.62-7.59(\mathrm{~m}, 2 \mathrm{H})$, 7.49-7.47 (m, $1 \mathrm{H})$, 7.20-7.17 (m, 1H), 7.12-7.04 (m, 2H), 6.77-6.74 (m, 2H), $6.50(\mathrm{~d}, J=2.2 \mathrm{~Hz}, 2 \mathrm{H}), 6.35(\mathrm{t}, J=2.3 \mathrm{~Hz}, 1 \mathrm{H}), 5.25(\mathrm{~d}, J$ $=17.1 \mathrm{~Hz}, 1 \mathrm{H}), 5.20(\mathrm{~d}, J=17.0 \mathrm{~Hz}, 1 \mathrm{H}), 3.78(\mathrm{~s}, 6 \mathrm{H}), 3.12-$ $2.98(\mathrm{~m}, 2 \mathrm{H}), 2.88-2.80(\mathrm{~m}, 1 \mathrm{H}), 2.78-2.75(\mathrm{~m}, 2 \mathrm{H}), 2.23-2.19$ (m, 1H), 2.13-2.03 (m, 1H) ppm.

${ }^{13}$ C-NMR $\left(100 \mathrm{MHz}, \mathrm{CD}_{2} \mathrm{Cl}_{2}\right) \delta=161.3,149.6,138.6,138.1,137.1,135.5,128.6,127.7,121.3$, $119.4,118.2,110.2,109.2,105.5,98.1,92.7,55.6,46.2,41.7,30.7,29.5,22.6$ ppm.

MS (EI) m/z (\%): 523 (96), 359 (100), 344 (4), 232 (14), 217 (43).

HRMS (ESI+) $m / z$ calculated for $\mathrm{C}_{27} \mathrm{H}_{26} \mathrm{NO}_{2} \mathrm{INa}\left(\mathrm{M}+\mathrm{Na}^{+}\right) 546.090047$, found 546.089902 .

The enantiomeric ratio was determined by reversed phase HPLC analysis using Daicel Chiralcel OJ-RH column: $\mathrm{MeCN}: \mathrm{H}_{2} \mathrm{O}=70: 30$, flow rate $1.0 \mathrm{~mL} / \mathrm{min}, \lambda=220 \mathrm{~nm}: \tau_{1}=17.26$ $\min , \tau_{2}=19.42 \min$. 


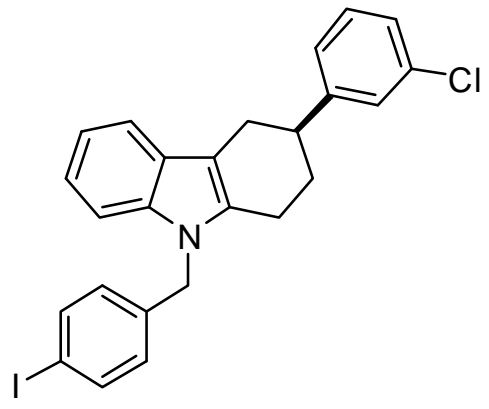

2n

Purification: Hexane:EtOAc (9:1). Colorless solid $48.2 \mathrm{mg}$ (0.097 mmol, 97\%).

${ }^{1}$ H-NMR (400 MHz, $\left.\mathrm{CD}_{2} \mathrm{Cl}_{2}\right) \delta=7.62-7.59(\mathrm{~m}, 2 \mathrm{H})$, 7.48-7.46 (m, $1 \mathrm{H}), 7.33(\mathrm{dd}, J=1.9,1.8 \mathrm{~Hz}, 1 \mathrm{H}), 7.28(\mathrm{dd}, J=7.8,7.3 \mathrm{~Hz}$, $1 \mathrm{H}), 7.25-7.21(\mathrm{~m}, 2 \mathrm{H}), 7.19(\mathrm{ddd}, J=8.2,1.2,1.0 \mathrm{~Hz}, 1 \mathrm{H})$, 7.12-7.04 (m, 2H), 6.77-6.74 (m, 2H), $5.25(\mathrm{~d}, J=17.0 \mathrm{~Hz}, 1 \mathrm{H})$, $5.20(\mathrm{~d}, J=17.1 \mathrm{~Hz}, 1 \mathrm{H}), 3.13-3.04(\mathrm{~m}, 2 \mathrm{H}), 2.87-2.74(\mathrm{~m}, 3 \mathrm{H})$, 2.24-2.18 (m, 1H), 2.14-2.03 (m, 1H) ppm.

${ }^{13} \mathrm{C}-\mathrm{NMR}\left(100 \mathrm{MHz}, \mathrm{CD}_{2} \mathrm{Cl}_{2}\right) \delta=149.2,138.6,138.1,137.1,135.4,134.4,130.1,128.6,127.7$, 127.6, 126.6, 125.8, 121.4, 119.5, 118.2, 110.0, 109.3, 92.7, 46.3, 41.0, 30.5, 29.4, 22.4 ppm. MS (EI) m/z (\%): 497 (80), 359 (100), 232 (18), 217 (54), 142 (9), 115 (9), 90 (21).

HRMS (ESI+) $m / z$ calculated for $\mathrm{C}_{25} \mathrm{H}_{21} \mathrm{NCIINa}\left(\mathrm{M}+\mathrm{Na}^{+}\right) 520.029946$, found 520.029908 .

The enantiomeric ratio was determined by reversed phase HPLC analysis using Daicel Chiralcel OD-RH column: $\mathrm{MeCN}: \mathrm{H}_{2} \mathrm{O}=70: 30$, flow rate $1.0 \mathrm{~mL} / \mathrm{min}, \lambda=220 \mathrm{~nm}: \tau_{1}=36.86$ $\min , \tau_{2}=39.63 \mathrm{~min}$.

(S)-9-(4-lodobenzyl)-3-(naphthalen-2-yl)-2,3,4,9-tetrahydro-1H-carbazole (2o)

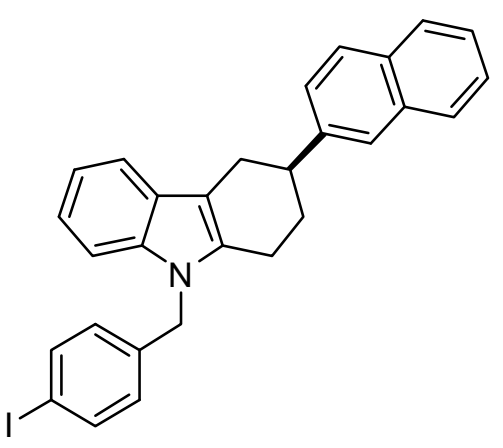

20

Purification: Hexane:EtOAc (9:1). Colorless solid $51.0 \mathrm{mg}$ (0.099 mmol, 99\%).

${ }^{1} \mathrm{H}-\mathrm{NMR}\left(400 \mathrm{MHz}, \mathrm{CD}_{2} \mathrm{Cl}_{2}\right) \delta=7.89-7.81(\mathrm{~m}, 3 \mathrm{H}), 7.78-7.77$ $(\mathrm{m}, 1 \mathrm{H}), 7.62-7.59(\mathrm{~m}, 2 \mathrm{H}), 7.53-7.50(\mathrm{~m}, 2 \mathrm{H}), 7.49-7.44(\mathrm{~m}$, $2 \mathrm{H})$, 7.22-7.19 (m, $1 \mathrm{H})$, 7.15-7.07 (m, $2 \mathrm{H})$, 6.79-6.76 (m, $2 \mathrm{H})$, $5.25(\mathrm{~d}, J=17.1 \mathrm{~Hz}, 1 \mathrm{H}), 5.20(\mathrm{~d}, J=17.1 \mathrm{~Hz}, 1 \mathrm{H}), 3.30-3.18$ $(\mathrm{m}, 2 \mathrm{H}), 3.02-2.95(\mathrm{~m}, 1 \mathrm{H}), 2.86-2.79(\mathrm{~m}, 2 \mathrm{H}), 2.36-2.29(\mathrm{~m}$, $1 \mathrm{H}), 2.27-2.17(\mathrm{~m}, 1 \mathrm{H}) \mathrm{ppm}$. 
${ }^{13} \mathrm{C}-\mathrm{NMR}\left(100 \mathrm{MHz}, \mathrm{CD}_{2} \mathrm{Cl}_{2}\right) \delta=138.1,137.1,135.6,134.1,132.7,128.6,128.2,127.9,127.9$, $127.8,126.5,126.3,125.6,125.3,121.3,119.4,118.2,110.3,109.3,92.7,46.2$, 41.4, 30.7, 29.5, $22.6 \mathrm{ppm}$.

MS (EI) m/z (\%): 513 (100), 359 (94), 232 (12), 217 (31), 154 (9).

HRMS (ESI+) $m / z$ calculated for $\mathrm{C}_{29} \mathrm{H}_{24} \mathrm{NINa}\left(\mathrm{M}+\mathrm{Na}^{+}\right)$536.085466, found 536.084749.

The enantiomeric ratio was determined by reversed phase HPLC analysis using Daicel Chiralcel OD-RH column: $\mathrm{MeCN}: \mathrm{H}_{2} \mathrm{O}=90: 10$, flow rate $1.0 \mathrm{~mL} / \mathrm{min}, \lambda=220 \mathrm{~nm}: \tau_{1}=9.39$ $\min , \tau_{2}=10.50 \min$.

(S)-9-(4-iodobenzyl)-3-methyl-2,3,4,9-tetrahydro-1H-carbazole (2p)

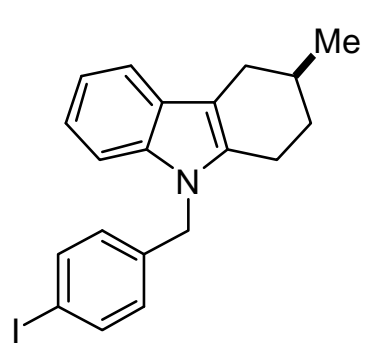

$2 p$

Purification: Hexane:EtOAc (19:1). Colorless solid $28.1 \mathrm{mg}(0.070$ $\mathrm{mmol}, 70 \%)$.

${ }^{1} \mathrm{H}-\mathrm{NMR}\left(500 \mathrm{MHz}, \mathrm{CDCl}_{3}\right) \delta=7.56(\mathrm{~d}, J=8.3 \mathrm{~Hz}, 2 \mathrm{H}), 7.50-7.48(\mathrm{~m}$, 1H), 7.15-7.13 (m, 1H), 7.11-7.07 (m, 2H), $6.71(\mathrm{~d}, J=8.3 \mathrm{~Hz}, 2 \mathrm{H}), 5.18$ (d, $J=17.3 \mathrm{~Hz}, 1 \mathrm{H}), 5.14(\mathrm{~d}, J=17.3 \mathrm{~Hz}, 1 \mathrm{H}), 2.88(\mathrm{dd}, J=15.5,4.7 \mathrm{~Hz}$, $1 \mathrm{H}), 2.66-2.56(\mathrm{~m}, 2 \mathrm{H}), 2.35-2.30(\mathrm{~m}, 1 \mathrm{H}), 1.96-1.94(\mathrm{~m}, 2 \mathrm{H}), 1.59-1.50$ (m, $1 \mathrm{H}), 1.13(\mathrm{~d}, J=6.5 \mathrm{~Hz}, 3 \mathrm{H}) \mathrm{ppm}$.

${ }^{13} \mathrm{C}-\mathrm{NMR}\left(100 \mathrm{MHz}, \mathrm{CDCl}_{3}\right) \delta=138.1,137.9,136.8,135.3,128.2,127.5,121.0,119.1,118.0$, $110.2,108.9,92.6,45.9,31.4,29.6,29.6,21.9,21.7$ ppm.

MS (EI) m/z (\%): 401 (100), 359 (35). 274 (5), 232 (10), 217 (25).

HRMS (ESI+): $m / z$ calculated for $\mathrm{C}_{20} \mathrm{H}_{21} \mathrm{NI}\left(\mathrm{M}+\mathrm{H}^{+}\right)$402.071318, found 402.071822 .

Mp: $121-122^{\circ} \mathrm{C}(\mathrm{MeOH})$.

$[\alpha]_{D}^{25}:-27.0^{\circ}, \mathrm{C}=0.23\left(\mathrm{CH}_{2} \mathrm{Cl}_{2}\right)$.

The enantiomeric ratio was determined by HPLC analysis using Daicel Chiralpak AD-3 column: $n$-heptane:i-PrOH $=99: 1$, flow rate $1.0 \mathrm{~mL} / \mathrm{min}, \lambda=220 \mathrm{~nm}: \tau_{1}=4.243 \mathrm{~min}, \tau_{2}=4.60$ $\min$ 


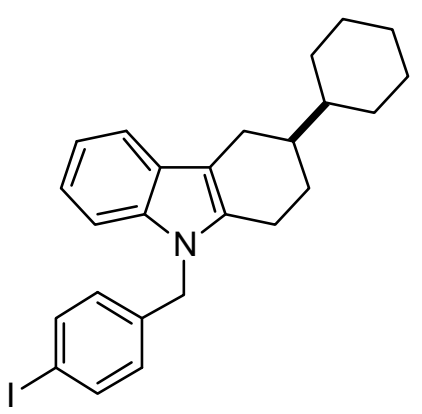

$2 q$

Purification: Hexane:EtOAc (9:1). Colorless solid $46.0 \mathrm{mg}(0.098$ $\mathrm{mmol}, 98 \%)$.

${ }^{1} \mathrm{H}-\mathrm{NMR}\left(400 \mathrm{MHz}, \mathrm{CD}_{2} \mathrm{Cl}_{2}\right) \delta=7.60-7.57(\mathrm{~m}, 2 \mathrm{H}), 7.47-7.44(\mathrm{~m}$, $1 \mathrm{H}), 7.15-7.12(\mathrm{~m}, 1 \mathrm{H}), 7.08-7.01(\mathrm{~m}, 2 \mathrm{H}), 6.75-6.72(\mathrm{~m}, 2 \mathrm{H}), 5.21$ (d, $J=17.0 \mathrm{~Hz}, 1 \mathrm{H}), 5.16(\mathrm{~d}, J=17.0 \mathrm{~Hz}, 1 \mathrm{H}), 2.86-2.81(\mathrm{~m}, 1 \mathrm{H})$, 2.73-2.68 (m, $1 \mathrm{H}), 2.62-2.55(\mathrm{~m}, 1 \mathrm{H}), 2.48-2.42(\mathrm{~m}, 1 \mathrm{H}), 2.08-2.04$ $(\mathrm{m}, 1 \mathrm{H}), 1.86-1.77(\mathrm{~m}, 4 \mathrm{H}), 1.71-1.65(\mathrm{~m}, 1 \mathrm{H}), 1.64-1.51(\mathrm{~m}, 2 \mathrm{H})$, 1.40-1.06 (m, 6H) ppm.

${ }^{13} \mathrm{C}-\mathrm{NMR}\left(100 \mathrm{MHz}, \mathrm{CD}_{2} \mathrm{Cl}_{2}\right) \delta=138.8,138.0,136.0,128.6,128.6,128.1,121.0,119.2,118.1$, 110.6, 109.1, 92.6, 46.2, 42.8, 40.7, 31.1, 30.6, 27.2, 27.2, 24.8, 22.8 ppm.

MS (EI) m/z (\%): 469 (100), 359 (41), 232 (6), 217 (19).

HRMS (ESI+) $m / z$ calculated for $\mathrm{C}_{25} \mathrm{H}_{28} \mathrm{NINa}\left(\mathrm{M}+\mathrm{Na}^{+}\right)$492.115866, found 492.116240 .

The enantiomeric ratio was determined by reversed phase HPLC analysis using Daicel Chiralcel OD-RH column: $\mathrm{MeCN}: \mathrm{H}_{2} \mathrm{O}=70: 30$, flow rate $1.0 \mathrm{~mL} / \mathrm{min}, \lambda=220 \mathrm{~nm}: \tau_{1}=38.30$ $\min , \tau_{2}=41.46 \min$.

(S)-3-(tert-Butyl)-9-(4-iodobenzyl)-2,3,4,9-tetrahydro-1H-carbazole (2r)

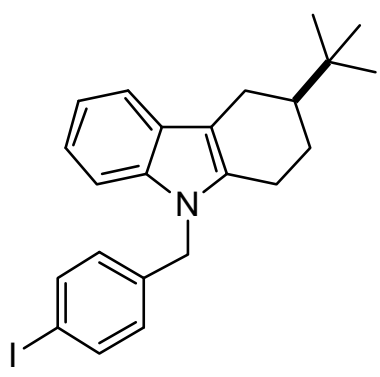

$2 r$

Purification: Hexane:EtOAc (9:1). Colorless solid $39.9 \mathrm{mg}(0.090$ $\mathrm{mmol}, 90 \%)$.

${ }^{1} \mathrm{H}-\mathrm{NMR}\left(400 \mathrm{MHz}, \mathrm{CDCl}_{3}\right) \delta=7.66-7.62(\mathrm{~m}, 2 \mathrm{H}), 7.26-7.22(\mathrm{~m}, 2 \mathrm{H})$, $7.11(\mathrm{~d}, J=8.3 \mathrm{~Hz}, 2 \mathrm{H}), 6.89-6.83(\mathrm{~m}, 3 \mathrm{H}), 4.57(\mathrm{~d}, J=14.9 \mathrm{~Hz}, 1 \mathrm{H})$, $4.47(\mathrm{~d}, J=14.9 \mathrm{~Hz}, 1 \mathrm{H}), 3.09-3.03(\mathrm{~m}, 1 \mathrm{H}), 2.67-2.61(\mathrm{~m}, 1 \mathrm{H}), 2.20$ (td, $J=13.4,4.8 \mathrm{~Hz}, 1 \mathrm{H}), 2.01-1.96(\mathrm{~m}, 1 \mathrm{H}), 1.74-1.69(\mathrm{~m}, 1 \mathrm{H}), 1.51$ (dt, $J=13.9,5.5 \mathrm{~Hz}, 1 \mathrm{H}), 1.25-1.09(\mathrm{~m}, 2 \mathrm{H}), 0.84(\mathrm{~s}, 9 \mathrm{H}), 0.76-0.65(\mathrm{~m}, 1 \mathrm{H}) \mathrm{ppm}$.

${ }^{13} \mathrm{C}-\mathrm{NMR}\left(100 \mathrm{MHz} \mathrm{CDCl}_{3}\right) \delta=177.2,150.1,138.3,136.9,133.3,128.6,119.8,115.5,91.9$, $59.6,47.0,34.7,32.0,29.2,27.7,27.2,26.5$ ppm. 
MS (EI) m/z (\%): 460 (47), 243 (100), 217 (13), 187 (5).

HRMS (ESI+) $\mathrm{m} / z$ calculated for $\mathrm{C}_{23} \mathrm{H}_{26} \mathrm{NINa}\left(\mathrm{M}+\mathrm{Na}^{+}\right)$466.100217, found 466.100293.

The enantiomeric ratio was determined by HPLC analysis using Daicel Chiralpak OD-3 column: $n$-heptane:i-PrOH $=99: 1$, flow rate $1.0 \mathrm{~mL} / \mathrm{min}, \lambda=220 \mathrm{~nm}: \tau_{1}=4.93 \mathrm{~min}, \tau_{2}=5.52$ $\min$.

(S)-9-(4-iodobenzyl)-2,3,4,9-tetrahydro-1H-carbazol-3-yl benzoate (2s)

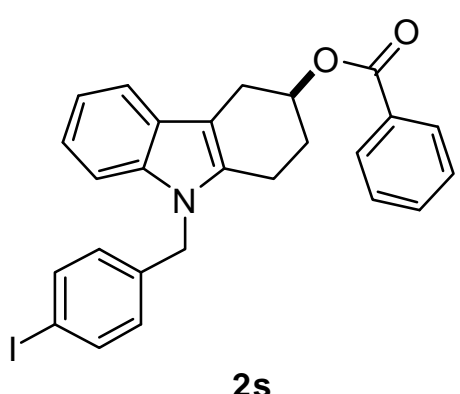

Purification: Hexane:EtOAc (9:1). Colorless solid $50.6 \mathrm{mg}(0.087$ $\mathrm{mmol}, 87 \%)$.

${ }^{1} \mathrm{H}-\mathrm{NMR}\left(500 \mathrm{MHz}, \mathrm{CDCl}_{3}\right) \delta=7.99(\mathrm{~d}, J=7.8 \mathrm{~Hz}, 2 \mathrm{H}), 7.58-7.50$ (m, 4H), $7.41(\mathrm{dd}, J=7.8,7.8 \mathrm{~Hz}, 2 \mathrm{H}), 7.19(\mathrm{~d}, J=7.9 \mathrm{~Hz}, 1 \mathrm{H})$, 7.16-7.10 (m, 2H), $6.70(\mathrm{~d}, J=8.1 \mathrm{~Hz}, 2 \mathrm{H}), 5.61-5.57(\mathrm{~m}, 1 \mathrm{H}), 5.24$ (d, $J=17.1 \mathrm{~Hz}, 1 \mathrm{H}), 5.19$ (d, $J=17.1 \mathrm{~Hz}, 1 \mathrm{H}), 3.26$ (dd, $J=15.9$, $4.2 \mathrm{~Hz}, 1 \mathrm{H}), 3.06(\mathrm{dd}, J=15.9,5.8 \mathrm{~Hz}, 1 \mathrm{H}), 2.85-2.74(\mathrm{~m}, 2 \mathrm{H}), 2.31-2.19(\mathrm{~m}, 2 \mathrm{H}) \mathrm{ppm}$.

${ }^{13} \mathrm{C}-\mathrm{NMR}\left(100 \mathrm{MHz}, \mathrm{CDCl}_{3}\right) \delta=166.3,137.9,137.9,137.0,134.0,133.0,130.7,129.7,128.4$, $128.1,127.4,121.5,119.5,118.1,109.1,107.1,92.7,70.3,46.0,27.8,27.3,19.4$ ppm. MS (EI) m/z (\%): 507 (60), 385 (100), 258 (10), 217 (30), 168 (25), 90 (10). HRMS (ESI+) $m / z$ calculated for $\mathrm{C}_{26} \mathrm{H}_{22} \mathrm{NO}_{2} \mathrm{INa}\left(\mathrm{M}+\mathrm{Na}^{+}\right) 530.058747$, found 530.058533 .

The enantiomeric ratio was determined by HPLC analysis using Daicel Chiralpak OD-3 column: $n$-heptane: $i$-PrOH $=80: 20$, flow rate $1.0 \mathrm{~mL} / \mathrm{min}, \lambda=220 \mathrm{~nm}: \tau_{1}=8.35 \mathrm{~min}, \tau_{2}=$ $14.14 \mathrm{~min}$. 


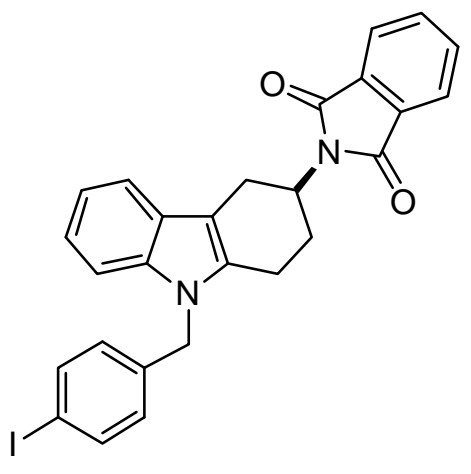

$2 t$

Purification: Hexane:EtOAc (1:1) then $\mathrm{CH}_{2} \mathrm{Cl}_{2}$ :EtOAc (1:1). Colorless solid $52.5 \mathrm{mg}$ (0.099 mmol, 99\%).

${ }^{1} \mathrm{H}-\mathrm{NMR}\left(500 \mathrm{MHz}, \mathrm{CD}_{2} \mathrm{Cl}_{2}\right) \delta=7.87-7.83(\mathrm{~m}, 2 \mathrm{H})$, 7.77-7.73 $(\mathrm{m}$, $2 \mathrm{H}), 7.62(\mathrm{~d}, J=8.4 \mathrm{~Hz}, 2 \mathrm{H}), 7.44(\mathrm{~d}, J=7.7 \mathrm{~Hz}, 1 \mathrm{H}), 7.18(\mathrm{~d}, J=$ $8.1 \mathrm{~Hz}, 1 \mathrm{H}$ ), 7.09 (ddd, $J=8.0,7.1,1.0 \mathrm{~Hz}, 1 \mathrm{H}$ ), 7.05 (ddd, $J=$ 7.4, 7.4, $0.9 \mathrm{~Hz}, 1 \mathrm{H}), 6.76(\mathrm{~d}, J=8.4 \mathrm{~Hz}, 2 \mathrm{H}), 5.25(\mathrm{~d}, J=17.1 \mathrm{~Hz}$, 1H), $5.20(\mathrm{~d}, J=17.1 \mathrm{~Hz}, 1 \mathrm{H}), 4.69-4.58(\mathrm{~m}, 1 \mathrm{H}), 3.50$ (dd, $J=14.1,11.7 \mathrm{~Hz}, 1 \mathrm{H}), 2.98(\mathrm{dd}, J=$ 14.6, $5.5 \mathrm{~Hz}, 1 \mathrm{H}), 2.91-2.79(\mathrm{~m}, 3 \mathrm{H}), 2.12-2.05(\mathrm{~m}, 1 \mathrm{H}) \mathrm{ppm}$.

${ }^{13} \mathrm{C}-\mathrm{NMR}\left(125 \mathrm{MHz}, \mathrm{CD}_{2} \mathrm{Cl}_{2}\right) \delta=168.7,138.4,138.1,137.3,134.5,134.3,132.5,128.5,127.5$, $123.3,121.5,119.5,118.2,109.3,108.8,92.7,48.4,46.3,27.1,25.1,22.4$ ppm.

MS (EI) $m / z$ (\%): 532 (54), 385 (100), 359 (10), 258 (12), 217 (29), 168 (35).

HRMS (ESI+) $m / z$ calculated for $\mathrm{C}_{27} \mathrm{H}_{21} \mathrm{~N}_{2} \mathrm{O}_{2} \mathrm{INa}\left(\mathrm{M}+\mathrm{Na}^{+}\right)$555.053992, found 555.054480 .

Mp: $256-257^{\circ} \mathrm{C}\left(\mathrm{CH}_{2} \mathrm{Cl}_{2}\right)$.

$[\alpha]_{D}^{25}:-2.80^{\circ}, \mathrm{c}=0.50\left(\mathrm{CH}_{2} \mathrm{Cl}_{2}, \mathrm{er}=93.5: 6.5\right)$.

The enantiomeric ratio was determined by HPLC analysis using Daicel Chiralpak AD-3 column: $n$-heptane:i-PrOH $=80: 20$, flow rate $1.0 \mathrm{~mL} / \mathrm{min}, \lambda=220 \mathrm{~nm}: \tau_{1}=9.69 \mathrm{~min}, \tau_{2}=$ 14.32 min. (The sample was dissolved in $\mathrm{CH}_{2} \mathrm{Cl}_{2}$ )

9-(4-iodobenzyl)-3-methyl-3-phenyl-2,3,4,9-tetrahydro-1H-carbazole (2u)

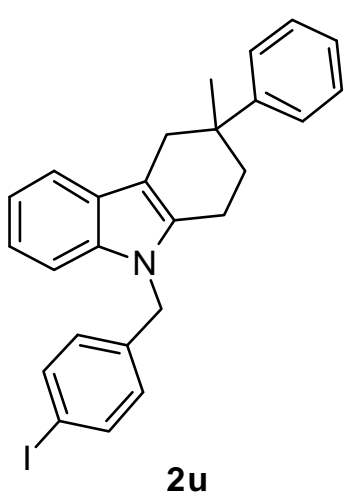

$2 u$
Purification: Hexane:EtOAc (20:1)

Colourless Solid $5.2 \mathrm{mg}$ (0.011 mmol, 11\%).

Starting material $1 \mathrm{u}$ recovered $(38.0 \mathrm{mg}, 77 \%)$

${ }^{1} \mathrm{H}-\mathrm{NMR}\left(500 \mathrm{MHz}, \mathrm{CD}_{2} \mathrm{Cl}_{2}\right) \delta=7.59-7.57(\mathrm{~m}, 1 \mathrm{H})$, 7.49-7.47 (m, 2H), 7.36-7.34 (m, 2H), 7.25-7.22 (m, 2H), 7.17-7.14 (m, 1H), 7.13-7.10 (m, 1H), 7.10-7.07 (m, 2H), $6.50(\mathrm{~d}, J=8.4 \mathrm{~Hz}, 2 \mathrm{H}), 5.11(\mathrm{~s}, 2 \mathrm{H}), 3.22$ 
(d, $J=15.8 \mathrm{~Hz}, 1 \mathrm{H}$ ), $2.86(\mathrm{~d}, J=15.8 \mathrm{~Hz}, 1 \mathrm{H}$ ), 2.60 (ddd, $J=16.2,5.2,4.8 \mathrm{~Hz}, 1 \mathrm{H}$ ), 2.33-2.28 (m, 1H), 2.26-2.20 (m, 1H), 2.08-2.01 (m, 1H), $1.43(\mathrm{~s}, 3 \mathrm{H}) \mathrm{ppm}$.

${ }^{13} \mathrm{C}-\mathrm{NMR}\left(125 \mathrm{MHz}, \mathrm{CD}_{2} \mathrm{Cl}_{2}\right) \delta=148.2,138.5,137.9,137.1,135.3,128.3,128.2,127.8,126.4$, 125.9, 121.1, 119.3, 118.1, 109.9, 109.2, 92.4, 46.0, 38.1, 36.2, 33.5, 29.8, 20.2 ppm.

MS (EI) m/z (\%): 477 (63), 359 (100), 344 (4), 232 (15), 217 (31).

HRMS (EI) $m / z$ calculated for $\mathrm{C}_{26} \mathrm{H}_{24} \mathrm{NI} 477.095347$, found 477.095027 .

The enantiomeric ratio was determined by reversed phase HPLC analysis using Daicel Chiralcel OJ-RH column: $\mathrm{MeCN}: \mathrm{H}_{2} \mathrm{O}=90: 10$, flow rate $1.0 \mathrm{~mL} / \mathrm{min}, \lambda=220 \mathrm{~nm}: \tau_{1}=6.97 \mathrm{~min}$, $\tau_{2}=15.27 \mathrm{~min}$.

(1R,2S)-4-(4-lodobenzyl)-1,2-diphenyl-1,2,3,4-tetrahydrocyclopenta[b]indole (2v)

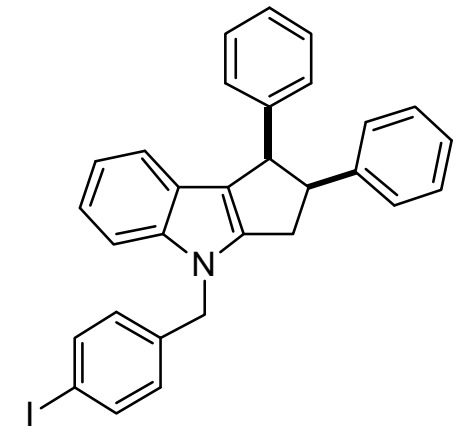

2v Purification: Hexane:EtOAc (15:1). Yellow solid $32.6 \mathrm{mg}(0.062$ $\mathrm{mmol}, 62 \%)$.

${ }^{1} \mathrm{H}-\mathrm{NMR}\left(400 \mathrm{MHz}, \mathrm{CD}_{2} \mathrm{Cl}_{2}\right) \delta=7.71-7.67(\mathrm{~m}, 2 \mathrm{H})$, 7.29-7.27 (m, $1 \mathrm{H}), 7.19(\mathrm{~d}, J=7.8 \mathrm{~Hz}, 1 \mathrm{H}), 7.10$ (ddd, $J=7.1,7.1,1.2 \mathrm{~Hz}, 1 \mathrm{H}$ ), 7.05-6.98 (m, 7H), $6.96(\mathrm{~d}, J=8.4 \mathrm{~Hz}, 2 \mathrm{H}), 6.91-6.89(\mathrm{~m}, 2 \mathrm{H})$, 6.75-6.72 (m, 2H), $5.36(\mathrm{~d}, J=16.5 \mathrm{~Hz}, 1 \mathrm{H}), 5.31(\mathrm{~d}, J=16.5 \mathrm{~Hz}$, $1 \mathrm{H}), 4.79(\mathrm{~d}, J=8.0 \mathrm{~Hz}, 1 \mathrm{H}), 4.54(\mathrm{dd}, J=16.0,8.0 \mathrm{~Hz}, 1 \mathrm{H}), 3.27-$ $3.16(\mathrm{~m}, 2 \mathrm{H}) \mathrm{ppm}$.

${ }^{13} \mathrm{C}-\mathrm{NMR}\left(100 \mathrm{MHz}, \mathrm{CD}_{2} \mathrm{Cl}_{2}\right) \delta=145.8,141.7,141.7,141.3,138.2,138.2,129.1,129.0,128.8$, $127.8,127.8,126.2,126.1,124.9,121.0,120.1,119.9,119.1,110.3,93.0,55.9,50.6,48.2$, $31.2 \mathrm{ppm}$

MS (EI) m/z (\%): 525 (100), 434 (19), 306 (14), 230 (16), 217 (38).

HRMS (ESI+) $m / z$ calculated for $\mathrm{C}_{30} \mathrm{H}_{24} \mathrm{NINa}\left(\mathrm{M}+\mathrm{Na}^{+}\right) 548.084563$, found 548.084784 .

The enantiomeric ratio was determined by reversed phase HPLC analysis using Daicel Chiralcel OJ-RH column: $\mathrm{MeCN}: \mathrm{H}_{2} \mathrm{O}=70: 30$, flow rate $1.0 \mathrm{~mL} / \mathrm{min}, \lambda=220 \mathrm{~nm}: \tau_{1}=31.81$ $\min , \tau_{2}=43.74 \mathrm{~min}$. 


\section{Large scale indolization and formal synthesis of $(S)$-Ramatroban}<smiles>O=C1c2ccccc2C(=O)N1C1CCC(=NN(Cc2ccc(I)cc2)c2ccccc2)CC1</smiles>

$1 \mathrm{t}$

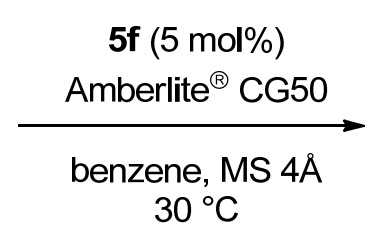

$30^{\circ} \mathrm{C}$

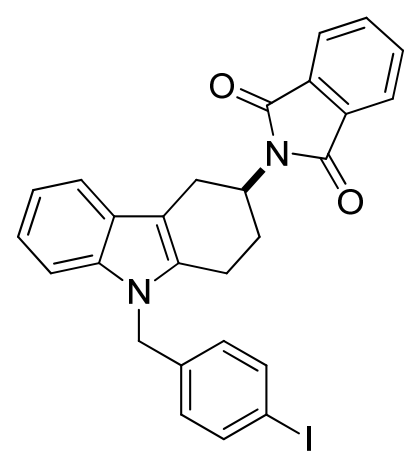

2t

$\mid \begin{gathered}\mathrm{H}_{2} \mathrm{NNH}_{2} \cdot \mathrm{H}_{2} \mathrm{O} \\ \mathrm{Et}_{3} \mathrm{~N} \\ \mathrm{CH}_{2} \mathrm{Cl}_{2}:-\mathrm{i}-\mathrm{PrOH} \\ (4: 1)\end{gathered}$

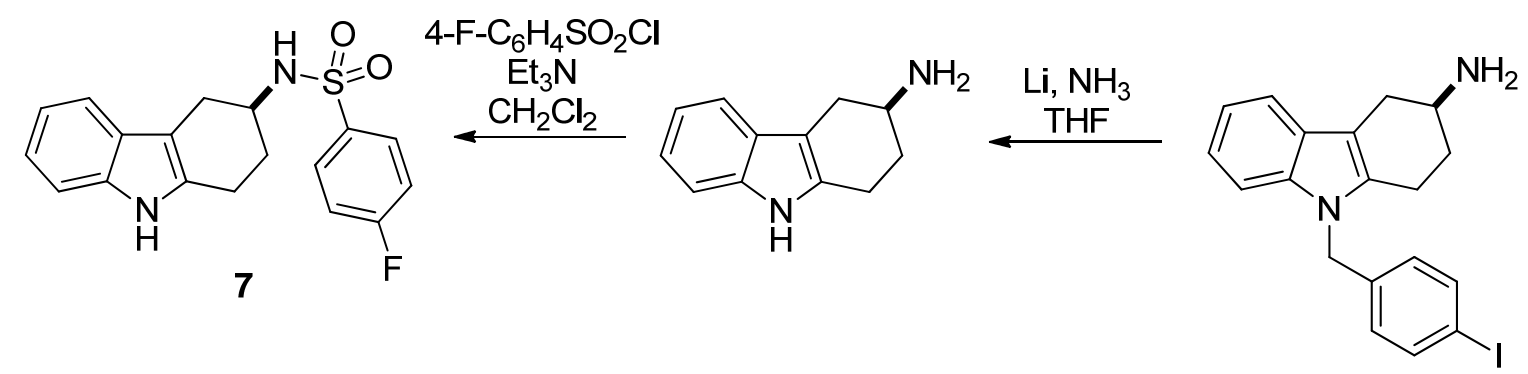

(S)-2-(9-(4-lodobenzyl)-2,3,4,9-tetrahydro-1H-carbazol-3-yl)isoindoline-1,3-dione (2t)

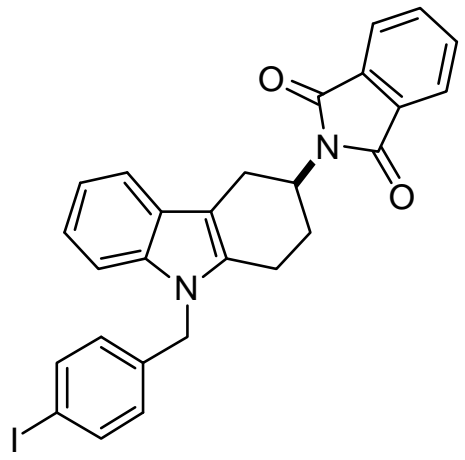

$2 \mathrm{t}$

A round-bottomed flask was charged with catalyst $\mathbf{5 f}(66.6$ $\mathrm{mg}, 0.01 \mathrm{mmol})$, Amberlite $^{\circledR}$ CG50 (4.00 g), hydrazone 1t $(1.10 \mathrm{~g}, 2.00 \mathrm{mmol})$ and molecular sieves $4 \AA$ (1.00 g). Under an atmosphere of argon, benzene $(20.0 \mathrm{~mL})$ was added and the resulting mixture was stirred at $30{ }^{\circ} \mathrm{C}$ for 6 days. The reaction mixture was then filtered and the residue washed thoroughly with $\mathrm{CH}_{2} \mathrm{Cl}_{2}$. The filtrate was concentrated in vacuo to give the crude product as a mixture of $\mathbf{2 t}$ (93.5:6.5

er) and catalyst $\mathbf{5 f}$. Recrystallization of this solid from boiling toluene $(27 \mathrm{~mL})$ gave the pure product as a fine colorless solid (856 $\mathrm{mg}, 1.61 \mathrm{mmol}, 80.4 \%$ ) with 94:6 er. The mother liquor was evaporated and subsequently subjected to $\mathrm{SiO}_{2}$ column chromatography eluting with hexane: EtOAc (1:1) then $\mathrm{CH}_{2} \mathrm{Cl}_{2}$ :EtOAc (1:1) then $\mathrm{CH}_{2} \mathrm{Cl}_{2}$ :MeOH (19:1). From this a further yield of $2 \mathrm{t}$ was obtained (109 $\mathrm{mg}, 0.205 \mathrm{mmol}, 10.2 \%$ ) with 93.5:6.5 er, resulting in an overall 
yield of $91 \%$ of $\mathbf{2 t}$. Spectroscopic data was in accordance with that reported above. Additionally recovery of catalyst $\mathbf{5} \mathbf{f}$ was achieved after acidification of the relevant fractions as described previously (36.7 $\mathrm{mg}, 0.055 \mathrm{mmol}, 55 \%)$.

(S)-9-(4-lodobenzyl)-2,3,4,9-tetrahydro-1H-carbazol-3-amine<smiles>N[C@H]1CCc2c(c3ccccc3n2Cc2ccc(I)cc2)C1</smiles>

In a Schlenk tube under argon, triethylamine (50 $\mu \mathrm{L}, 0.361 \mathrm{mmol})$ and hydrazine hydrate $(26 \mu \mathrm{L}, 0.542 \mathrm{mmol})$ were added to a suspension of phthalimide 2t (96 mg, $0.181 \mathrm{mmol})$ in $\mathrm{CH}_{2} \mathrm{Cl}_{2}(2 \mathrm{~mL})$ and $i-\operatorname{PrOH}(0.5$ $\mathrm{mL}$ ). The mixture was stirred at $40{ }^{\circ} \mathrm{C}$ for $48 \mathrm{~h}$ before the solvent was evaporated in vacuo. The resulting solid was used directly in the next step without purification.

(S)-2,3,4,9-Tetrahydro-1H-carbazol-3-amine

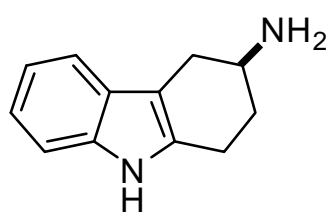

A suspension of (S)-9-(4-lodobenzyl)-2,3,4,9-tetrahydro-1H-carbazol-3amine (0.181 mmol; assumed quantitative from $2 \mathrm{t})$ in THF $(1.5 \mathrm{~mL})$ was cooled to $-78{ }^{\circ} \mathrm{C}$ in a Schlenk tube. Ammonia $(3 \mathrm{~mL})$ was condensed into the flask and lithium powder $(12.6 \mathrm{mg}, 1.81 \mathrm{mmol})$ was then added. The resulting deep blue solution was stirred at $-78{ }^{\circ} \mathrm{C}$ for $3 \mathrm{~h}$. The reaction was quenched by the addition of EtOH $(500 \mu \mathrm{L})$ at $-78{ }^{\circ} \mathrm{C}$. The reaction was then allowed to warm to room temperature, allowing the ammonia to evaporate. The mixture was diluted with $10 \%$ aqueous $\mathrm{HCl}(5 \mathrm{~mL})$ and subsequently washed with $\mathrm{Et}_{2} \mathrm{O}(2 \times 5 \mathrm{~mL})$. The aqueous layer was then cooled in ice and basified to $\mathrm{pH} 10$ with $\mathrm{NaOH}$. This basic aqueous mixture was then extracted with $\mathrm{CH}_{2} \mathrm{Cl}_{2}(3 \mathrm{x}$ $5 \mathrm{~mL}$ ). The combined $\mathrm{CH}_{2} \mathrm{Cl}_{2}$ extracts were then dried over $\mathrm{MgSO}_{4}$ and concentrated in vacuo to yield a colorless solid $(42.0 \mathrm{mg}$ ) which was used directly in the next step without purification. 
${ }^{1} \mathrm{H}-\mathrm{NMR}\left(300 \mathrm{MHz}, \mathrm{CD}_{2} \mathrm{Cl}_{2}\right) \delta=8.13(\mathrm{~s}$, broad, $1 \mathrm{H}), 7.42(\mathrm{~d}, J=7.3 \mathrm{~Hz}, 1 \mathrm{H}), 7.28-7.26(\mathrm{~m}, 1 \mathrm{H})$, 7.11-7.00 (m, 2H), 3.29-3.22 (m, 1H), 3.03-2.96 (m, 1H), 2.84-2.79 (m, 2H), 2.46-2.38 (m, $1 \mathrm{H})$, 2.10-2.01 (m, 1H), 1.85-1.75 (m, 1H) ppm.

MS (EI) m/z (\%): 186 (30), 168 (15), 143 (100).

HRMS (EI) $m / z$ calculated for $\mathrm{C}_{12} \mathrm{H}_{14} \mathrm{~N}_{2}$ (M) 186.115685, found 186.115542 .

(S)-4-Fluoro- $N$-(2,3,4,9-tetrahydro-1H-carbazol-3-yl)benzenesulfonamide (7)

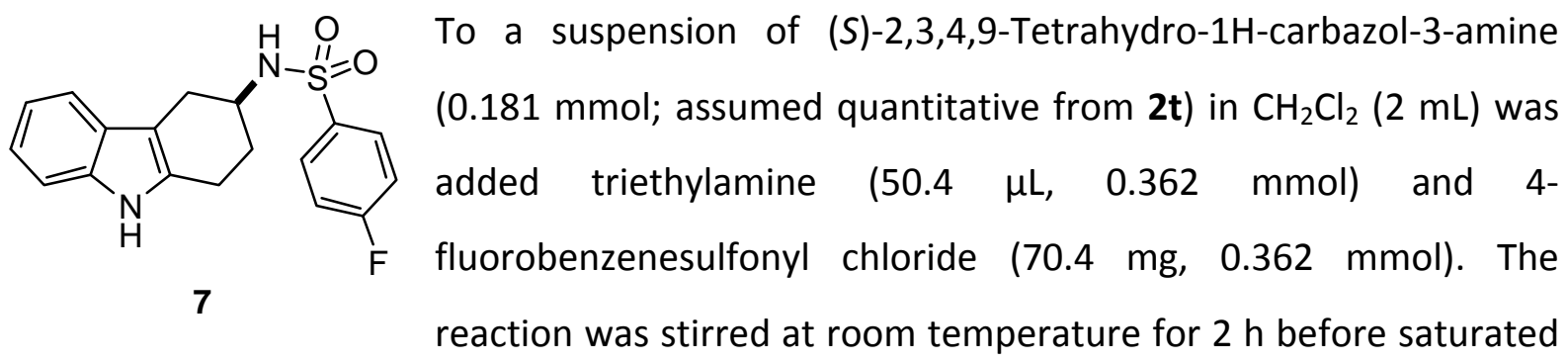
aqueous $\mathrm{NaHCO}_{3}(4 \mathrm{~mL})$ was added. The organic phase was separated and the aqueous phase extracted with $\mathrm{CH}_{2} \mathrm{Cl}_{2}(2 \times 4 \mathrm{~mL})$. The combined organic extracts were dried over $\mathrm{MgSO}_{4}$ and concentrated in vacuo. The crude material was purified by flash chromatography on $\mathrm{SiO}_{2}$ eluting with hexane:EtOAc (90:10 then 70:30) to give sulfonamide 7 as a colorless solid $45.4 \mathrm{mg}$ ( $0.132 \mathrm{mmol}, 73 \%$ over 3 steps from $2 \mathrm{t}$; 93:7 er).

${ }^{1} \mathrm{H}-\mathrm{NMR}\left(400 \mathrm{MHz}, \mathrm{CD}_{2} \mathrm{Cl}_{2}\right) \delta=7.83-7.79(\mathrm{~m}, 3 \mathrm{H})$, 7.19-7.18 (m, 2H), 7.16-7.11 (m, 2H), 7.03$6.99(\mathrm{~m}, 1 \mathrm{H}), 6.95-6.91(\mathrm{~m}, 1 \mathrm{H}), 4.73(\mathrm{~d}, J=8.0 \mathrm{~Hz}, 1 \mathrm{H}), 3.71-3.66(\mathrm{~m}, 1 \mathrm{H}), 2.82-2.77(\mathrm{~m}, 1 \mathrm{H})$, 2.73-2.70 (m, 2H), 2.45-2-39 (m, 1H), 1.97-1.84 (m, 2H) ppm.

${ }^{13} \mathrm{C}-\mathrm{NMR}\left(100 \mathrm{MHz}, \mathrm{CD}_{2} \mathrm{Cl}_{2}\right) \delta=165.5(\mathrm{~d}, J=252 \mathrm{~Hz}), 137.7,136.6,133.0,130.1$ (d, $J=9.4$ $\mathrm{Hz}), 127.8,121.8,119.6,117.8,116.7$ (d, $J=22.6 \mathrm{~Hz}$ ), 110.8, 106.9, 50.0, 29.6, 28.8, 20.7 ppm.

MS (EI) m/z (\%): 344 (90), 185 (90), 169 (100), 156 (25), 143 (90).

HRMS (ESI+) $m / z$ calculated for $\mathrm{C}_{18} \mathrm{H}_{17} \mathrm{~N}_{2} \mathrm{O}_{2} \mathrm{FSNa}\left(\mathrm{M}+\mathrm{Na}^{+}\right)$367.088700, found 367.088723 .

HPLC The enantiomeric ratio was determined by reversed phase HPLC analysis using Daicel Chiralcel OJ-RH column: $\mathrm{MeCN}: \mathrm{H}_{2} \mathrm{O}=50: 50$, flow rate $1.0 \mathrm{~mL} / \mathrm{min}, \lambda=220 \mathrm{~nm}: \tau_{1}=7.52 \mathrm{~min}$, $\tau_{2}=9.28 \mathrm{~min}$.

$[\alpha]_{\mathrm{D}}^{25}:-35.2^{\circ}, \mathrm{C}=0.25\left(\mathrm{CHCl}_{3}\right)$. 


\section{X-ray crystallographic data for compound $2 \mathrm{~b}$}

\section{Table 1. Crystal data and structure refinement.}

Identification code

Empirical formula

Color

Formula weight

Temperature

Wavelength

Crystal system

Space group

Unit cell dimensions

Volume

Z

Density (calculated)

Absorption coefficient

$\mathrm{F}(000)$

Crystal size

$\theta$ range for data collection

Index ranges

Reflections collected

Independent reflections

Reflections with $\mathrm{I}>2 \sigma(\mathrm{I})$

Completeness to $\theta=27.50^{\circ}$

Absorption correction

Max. and min. transmission

Refinement method

Data / restraints / parameters

Goodness-of-fit on $\mathrm{F}^{2}$

Final $R$ indices $[\mathrm{I}>2 \sigma(\mathrm{I})]$

$\mathrm{R}$ indices (all data)

Absolute structure parameter

Largest diff. peak and hole
7395

$\mathrm{C}_{25} \mathrm{H}_{22}$ I N

colourless

$463.34 \mathrm{~g} \cdot \mathrm{mol}^{-1}$

$100 \mathrm{~K}$

$0.71073 \AA$

ORTHORHOMBIC

$\mathrm{P} 22_{1} 2_{1}$, (no. 19)

$\mathrm{a}=9.3325(9) \AA \quad \alpha=90^{\circ}$.

$\mathrm{b}=13.9742(14) \AA \quad \beta=90^{\circ}$.

$\mathrm{c}=30.898(3) \AA \quad \gamma=90^{\circ}$.

4029.5(7) $\AA^{3}$

8

$1.528 \mathrm{Mg} \cdot \mathrm{m}^{-3}$

$1.598 \mathrm{~mm}^{-1}$

$1856 \mathrm{e}$

$0.64 \times 0.05 \times 0.04 \mathrm{~mm}^{3}$

1.32 to $31.03^{\circ}$.

$-13 \leq \mathrm{h} \leq 13,-20 \leq \mathrm{k} \leq 20,-44 \leq \mathrm{l} \leq 44$

147552

$12832\left[\mathrm{R}_{\mathrm{int}}=0.0567\right]$

11149

$100.0 \%$

Gaussian

0.94 and 0.58

Full-matrix least-squares on $\mathrm{F}^{2}$

12832 / 0 / 487

1.072

$\mathrm{R}_{1}=0.0298 \quad \mathrm{wR}^{2}=0.0743$

$\mathrm{R}_{1}=0.0398 \quad \mathrm{wR}^{2}=0.0832$

$-0.002(14)$

0.625 and $-1.222 \mathrm{e} \cdot \AA^{-3}$ 
Table 2. Atomic coordinates and equivalent isotropic displacement parameters $\left(\AA^{2}\right)$.

$\mathrm{U}_{\mathrm{eq}}$ is defined as one third of the trace of the orthogonalized $\mathrm{U}_{\mathrm{ij}}$ tensor.

\begin{tabular}{|c|c|c|c|c|}
\hline & $\mathrm{X}$ & $\mathrm{y}$ & $\mathrm{Z}$ & $\mathrm{U}_{\mathrm{eq}}$ \\
\hline $\mathrm{I}(1)$ & $1.0763(1)$ & $0.7526(1)$ & $0.4893(1)$ & $0.027(1)$ \\
\hline $\mathrm{I}(2)$ & $0.5710(1)$ & $0.2432(1)$ & $0.4930(1)$ & $0.025(1)$ \\
\hline $\mathrm{N}(1)$ & $0.5651(3)$ & $0.5698(1)$ & $0.3417(1)$ & $0.019(1)$ \\
\hline $\mathrm{N}(2)$ & $0.0692(2)$ & $0.4306(1)$ & $0.3439(1)$ & $0.017(1)$ \\
\hline $\mathrm{C}(1)$ & $0.4692(3)$ & $0.6451(2)$ & $0.3464(1)$ & $0.018(1)$ \\
\hline $\mathrm{C}(2)$ & 0.3991(3) & $0.6778(2)$ & $0.3834(1)$ & $0.021(1)$ \\
\hline C(3) & $0.3090(3)$ & $0.7556(2)$ & $0.3785(1)$ & $0.023(1)$ \\
\hline C(4) & $0.2879(3)$ & $0.7994(2)$ & $0.3378(1)$ & $0.023(1)$ \\
\hline $\mathrm{C}(5)$ & $0.3578(3)$ & $0.7665(2)$ & $0.3013(1)$ & $0.020(1)$ \\
\hline C(6) & $0.4525(3)$ & $0.6887(2)$ & $0.3052(1)$ & $0.017(1)$ \\
\hline C(7) & $0.5465(3)$ & $0.6399(2)$ & $0.2762(1)$ & $0.017(1)$ \\
\hline C(8) & $0.5716(3)$ & $0.6542(2)$ & $0.2285(1)$ & $0.019(1)$ \\
\hline C(9) & $0.6379(3)$ & $0.5632(2)$ & $0.2091(1)$ & $0.019(1)$ \\
\hline$C(10)$ & $0.7675(3)$ & $0.5328(2)$ & $0.2367(1)$ & $0.025(1)$ \\
\hline $\mathrm{C}(11)$ & $0.7215(3)$ & $0.5001(2)$ & $0.2821(1)$ & $0.024(1)$ \\
\hline $\mathrm{C}(12)$ & $0.6134(3)$ & $0.5693(2)$ & $0.2992(1)$ & $0.019(1)$ \\
\hline$C(13)$ & $0.6234(3)$ & $0.5141(2)$ & $0.3774(1)$ & $0.022(1)$ \\
\hline $\mathrm{C}(14)$ & $0.7360(3)$ & $0.5685(2)$ & $0.4031(1)$ & $0.018(1)$ \\
\hline$C(15)$ & $0.7565(3)$ & $0.5470(2)$ & $0.4469(1)$ & $0.021(1)$ \\
\hline $\mathrm{C}(16)$ & $0.8565(3)$ & $0.5976(2)$ & $0.4714(1)$ & $0.022(1)$ \\
\hline $\mathrm{C}(17)$ & $0.9342(3)$ & $0.6705(2)$ & $0.4518(1)$ & $0.019(1)$ \\
\hline$C(18)$ & $0.9169(3)$ & $0.6919(2)$ & $0.4084(1)$ & $0.018(1)$ \\
\hline C(19) & $0.8174(3)$ & $0.6401(2)$ & $0.3843(1)$ & $0.018(1)$ \\
\hline C(20) & $0.6724(3)$ & $0.5741(2)$ & $0.1615(1)$ & $0.019(1)$ \\
\hline $\mathrm{C}(21)$ & $0.5760(3)$ & $0.5390(2)$ & $0.1311(1)$ & $0.022(1)$ \\
\hline$C(22)$ & $0.6042(3)$ & $0.5444(2)$ & $0.0872(1)$ & $0.024(1)$ \\
\hline C(23) & 0.7297(3) & $0.5860(2)$ & $0.0725(1)$ & $0.025(1)$ \\
\hline$C(24)$ & $0.8263(3)$ & $0.6237(2)$ & $0.1024(1)$ & $0.025(1)$ \\
\hline C(25) & $0.7970(3)$ & $0.6173(2)$ & $0.1465(1)$ & $0.023(1)$ \\
\hline C(31) & $-0.0267(3)$ & $0.3565(2)$ & $0.3495(1)$ & $0.016(1)$ \\
\hline C(32) & $-0.0984(3)$ & $0.3264(2)$ & $0.3868(1)$ & $0.020(1)$ \\
\hline C(33) & $-0.1902(3)$ & $0.2488(2)$ & $0.3831(1)$ & $0.024(1)$ \\
\hline C(34) & $-0.2110(3)$ & $0.2028(2)$ & $0.3432(1)$ & $0.023(1)$ \\
\hline C(35) & $-0.1404(3)$ & $0.2327(2)$ & $0.3061(1)$ & $0.019(1)$ \\
\hline
\end{tabular}




$\begin{array}{lllll}\mathrm{C}(36) & -0.0444(3) & 0.3101(2) & 0.3090(1) & 0.017(1) \\ \mathrm{C}(37) & 0.0489(3) & 0.3571(2) & 0.2790(1) & 0.016(1) \\ \mathrm{C}(38) & 0.0726(3) & 0.3378(2) & 0.2318(1) & 0.017(1) \\ \mathrm{C}(39) & 0.2012(3) & 0.3943(2) & 0.2143(1) & 0.021(1) \\ \mathrm{C}(40) & 0.2054(3) & 0.4958(2) & 0.2332(1) & 0.023(1) \\ \mathrm{C}(41) & 0.2210(3) & 0.4975(2) & 0.2824(1) & 0.022(1) \\ \mathrm{C}(42) & 0.1155(3) & 0.4291(2) & 0.3011(1) & 0.017(1) \\ \mathrm{C}(43) & 0.1303(3) & 0.4862(2) & 0.3790(1) & 0.019(1) \\ \mathrm{C}(44) & 0.2393(3) & 0.4310(2) & 0.4052(1) & 0.015(1) \\ \mathrm{C}(45) & 0.2654(3) & 0.4559(2) & 0.4483(1) & 0.021(1) \\ \mathrm{C}(46) & 0.3627(3) & 0.4045(2) & 0.4731(1) & 0.021(1) \\ \mathrm{C}(47) & 0.4347(3) & 0.3271(2) & 0.4548(1) & 0.018(1) \\ \mathrm{C}(48) & 0.4129(3) & 0.3026(2) & 0.4117(1) & 0.018(1) \\ \mathrm{C}(49) & 0.3154(3) & 0.3547(2) & 0.3871(1) & 0.016(1) \\ \mathrm{C}(50) & 0.2031(3) & 0.3982(2) & 0.1650(1) & 0.019(1) \\ \mathrm{C}(51) & 0.3184(3) & 0.3605(2) & 0.1425(1) & 0.020(1) \\ \mathrm{C}(52) & 0.3245(3) & 0.3675(2) & 0.0976(1) & 0.022(1) \\ \mathrm{C}(53) & 0.2175(3) & 0.4116(2) & 0.0746(1) & 0.023(1) \\ \mathrm{C}(54) & 0.1007(3) & 0.4494(2) & 0.0966(1) & 0.025(1) \\ \mathrm{C}(55) & 0.0941(3) & 0.4430(2) & 0.1416(1) & 0.023(1)\end{array}$


Table 3. Bond lengths $[\AA]]$ and angles $\left[^{\circ}\right]$.

\begin{tabular}{|c|c|c|c|}
\hline $\mathrm{I}(1)-\mathrm{C}(17)$ & $2.101(2)$ & $\mathrm{I}(2)-\mathrm{C}(47)$ & $2.094(2)$ \\
\hline $\mathrm{N}(1)-\mathrm{C}(1)$ & $1.388(3)$ & $\mathrm{N}(1)-\mathrm{C}(12)$ & 1.388(3) \\
\hline $\mathrm{N}(1)-\mathrm{C}(13)$ & $1.456(3)$ & $\mathrm{N}(2)-\mathrm{C}(31)$ & $1.379(3)$ \\
\hline N(2)-C(42) & $1.389(3)$ & N(2)-C(43) & $1.452(3)$ \\
\hline $\mathrm{C}(1)-\mathrm{C}(2)$ & $1.396(4)$ & $C(1)-C(6)$ & $1.417(3)$ \\
\hline $\mathrm{C}(2)-\mathrm{C}(3)$ & $1.383(4)$ & $\mathrm{C}(3)-\mathrm{C}(4)$ & $1.412(3)$ \\
\hline$C(4)-C(5)$ & $1.383(4)$ & $C(5)-C(6)$ & $1.407(3)$ \\
\hline$C(6)-C(7)$ & $1.428(3)$ & C(7)-C(8) & $1.504(3)$ \\
\hline C(7)-C(12) & $1.369(3)$ & $\mathrm{C}(8)-\mathrm{C}(9)$ & $1.535(3)$ \\
\hline $\mathrm{C}(9)-\mathrm{C}(10)$ & $1.538(4)$ & C(9)-C(20) & $1.515(3)$ \\
\hline $\mathrm{C}(10)-\mathrm{C}(11)$ & $1.536(4)$ & $\mathrm{C}(11)-\mathrm{C}(12)$ & $1.495(4)$ \\
\hline $\mathrm{C}(13)-\mathrm{C}(14)$ & $1.522(4)$ & $C(14)-C(15)$ & 1.397(3) \\
\hline C(14)-C(19) & $1.384(3)$ & $\mathrm{C}(15)-\mathrm{C}(16)$ & $1.394(4)$ \\
\hline $\mathrm{C}(16)-\mathrm{C}(17)$ & $1.388(4)$ & $\mathrm{C}(17)-\mathrm{C}(18)$ & $1.385(3)$ \\
\hline C(18)-C(19) & 1.394(3) & $\mathrm{C}(20)-\mathrm{C}(21)$ & $1.390(3)$ \\
\hline $\mathrm{C}(20)-\mathrm{C}(25)$ & $1.390(4)$ & $\mathrm{C}(21)-\mathrm{C}(22)$ & 1.385(3) \\
\hline $\mathrm{C}(22)-\mathrm{C}(23)$ & $1.384(4)$ & $C(23)-C(24)$ & $1.394(4)$ \\
\hline $\mathrm{C}(24)-\mathrm{C}(25)$ & $1.392(4)$ & $\mathrm{C}(31)-\mathrm{C}(32)$ & 1.397(3) \\
\hline C(31)-C(36) & $1.419(3)$ & C(32)-C(33) & $1.386(4)$ \\
\hline C(33)-C(34) & $1.405(4)$ & $\mathrm{C}(34)-\mathrm{C}(35)$ & 1.386(3) \\
\hline C(35)-C(36) & $1.406(3)$ & C(36)-C(37) & 1.433(3) \\
\hline C(37)-C(38) & $1.500(3)$ & $\mathrm{C}(37)-\mathrm{C}(42)$ & $1.367(3)$ \\
\hline C(38)-C(39) & $1.535(3)$ & C(39)-C(40) & $1.534(3)$ \\
\hline C(39)-C(50) & $1.525(3)$ & $\mathrm{C}(40)-\mathrm{C}(41)$ & $1.528(4)$ \\
\hline$C(41)-C(42)$ & $1.489(4)$ & $C(43)-C(44)$ & 1.511(3) \\
\hline$C(44)-C(45)$ & 1.398(3) & $\mathrm{C}(44)-\mathrm{C}(49)$ & 1.397(3) \\
\hline$C(45)-C(46)$ & $1.390(4)$ & $C(46)-C(47)$ & 1.393(3) \\
\hline $\mathrm{C}(47)-\mathrm{C}(48)$ & 1.389(3) & $\mathrm{C}(48)-\mathrm{C}(49)$ & 1.392(3) \\
\hline$C(50)-C(51)$ & $1.385(4)$ & $C(50)-C(55)$ & $1.397(4)$ \\
\hline C(51)-C(52) & 1.391(3) & $\mathrm{C}(52)-\mathrm{C}(53)$ & $1.372(4)$ \\
\hline C(53)-C(54) & $1.389(4)$ & $C(54)-C(55)$ & $1.393(4)$ \\
\hline $\mathrm{C}(1)-\mathrm{N}(1)-\mathrm{C}(13)$ & $124.6(2)$ & $\mathrm{C}(12)-\mathrm{N}(1)-\mathrm{C}(1)$ & 108.16(19) \\
\hline $\mathrm{C}(12)-\mathrm{N}(1)-\mathrm{C}(13)$ & $126.3(2)$ & $\mathrm{C}(31)-\mathrm{N}(2)-\mathrm{C}(42)$ & 108.12(19) \\
\hline $\mathrm{C}(31)-\mathrm{N}(2)-\mathrm{C}(43)$ & $124.2(2)$ & $\mathrm{C}(42)-\mathrm{N}(2)-\mathrm{C}(43)$ & $126.6(2)$ \\
\hline $\mathrm{N}(1)-\mathrm{C}(1)-\mathrm{C}(2)$ & $129.4(2)$ & $\mathrm{N}(1)-\mathrm{C}(1)-\mathrm{C}(6)$ & $107.7(2)$ \\
\hline$C(2)-C(1)-C(6)$ & $122.9(2)$ & $\mathrm{C}(3)-\mathrm{C}(2)-\mathrm{C}(1)$ & 116.9(2) \\
\hline$C(2)-C(3)-C(4)$ & $121.6(2)$ & $\mathrm{C}(5)-\mathrm{C}(4)-\mathrm{C}(3)$ & $121.1(2)$ \\
\hline
\end{tabular}




\begin{tabular}{|c|c|c|c|}
\hline$C(4)-C(5)-C(6)$ & $118.8(2)$ & $\mathrm{C}(1)-\mathrm{C}(6)-\mathrm{C}(7)$ & $106.9(2)$ \\
\hline$C(5)-C(6)-C(1)$ & $118.7(2)$ & $\mathrm{C}(5)-\mathrm{C}(6)-\mathrm{C}(7)$ & $134.4(2)$ \\
\hline$C(6)-C(7)-C(8)$ & $130.4(2)$ & $\mathrm{C}(12)-\mathrm{C}(7)-\mathrm{C}(6)$ & $107.3(2)$ \\
\hline $\mathrm{C}(12)-\mathrm{C}(7)-\mathrm{C}(8)$ & $122.3(2)$ & $\mathrm{C}(7)-\mathrm{C}(8)-\mathrm{C}(9)$ & 109.57(19) \\
\hline $\mathrm{C}(8)-\mathrm{C}(9)-\mathrm{C}(10)$ & 109.3(2) & $\mathrm{C}(20)-\mathrm{C}(9)-\mathrm{C}(8)$ & 112.44(19) \\
\hline$C(20)-C(9)-C(10)$ & $113.4(2)$ & $\mathrm{C}(11)-\mathrm{C}(10)-\mathrm{C}(9)$ & $111.6(2)$ \\
\hline$C(12)-C(11)-C(10)$ & 108.6(2) & $\mathrm{N}(1)-\mathrm{C}(12)-\mathrm{C}(11)$ & $124.0(2)$ \\
\hline $\mathrm{C}(7)-\mathrm{C}(12)-\mathrm{N}(1)$ & $109.9(2)$ & $\mathrm{C}(7)-\mathrm{C}(12)-\mathrm{C}(11)$ & $126.2(2)$ \\
\hline $\mathrm{N}(1)-\mathrm{C}(13)-\mathrm{C}(14)$ & 112.74(19) & $\mathrm{C}(15)-\mathrm{C}(14)-\mathrm{C}(13)$ & 119.5(2) \\
\hline $\mathrm{C}(19)-\mathrm{C}(14)-\mathrm{C}(13)$ & $121.3(2)$ & $\mathrm{C}(19)-\mathrm{C}(14)-\mathrm{C}(15)$ & $119.2(2)$ \\
\hline$C(16)-C(15)-C(14)$ & $120.5(2)$ & $\mathrm{C}(17)-\mathrm{C}(16)-\mathrm{C}(15)$ & 119.1(2) \\
\hline C(16)-C(17)-I(1) & 119.43(17) & $\mathrm{C}(18)-\mathrm{C}(17)-\mathrm{I}(1)$ & 119.27(19) \\
\hline C(18)-C(17)-C(16) & 121.3(2) & $\mathrm{C}(17)-\mathrm{C}(18)-\mathrm{C}(19)$ & $118.9(2)$ \\
\hline C(14)-C(19)-C(18) & 121.1(2) & $\mathrm{C}(21)-\mathrm{C}(20)-\mathrm{C}(9)$ & $118.9(2)$ \\
\hline $\mathrm{C}(21)-\mathrm{C}(20)-\mathrm{C}(25)$ & $118.0(2)$ & $\mathrm{C}(25)-\mathrm{C}(20)-\mathrm{C}(9)$ & 123.1(2) \\
\hline $\mathrm{C}(22)-\mathrm{C}(21)-\mathrm{C}(20)$ & 121.3(3) & $\mathrm{C}(23)-\mathrm{C}(22)-\mathrm{C}(21)$ & 120.3(3) \\
\hline C(22)-C(23)-C(24) & 119.4(3) & $\mathrm{C}(25)-\mathrm{C}(24)-\mathrm{C}(23)$ & 119.7(3) \\
\hline C(20)-C(25)-C(24) & 121.3(3) & $\mathrm{N}(2)-\mathrm{C}(31)-\mathrm{C}(32)$ & $129.9(2)$ \\
\hline $\mathrm{N}(2)-\mathrm{C}(31)-\mathrm{C}(36)$ & $107.9(2)$ & $\mathrm{C}(32)-\mathrm{C}(31)-\mathrm{C}(36)$ & 122.2(2) \\
\hline C(33)-C(32)-C(31) & $117.7(2)$ & $\mathrm{C}(32)-\mathrm{C}(33)-\mathrm{C}(34)$ & $121.0(2)$ \\
\hline C(35)-C(34)-C(33) & $121.5(2)$ & $\mathrm{C}(34)-\mathrm{C}(35)-\mathrm{C}(36)$ & 118.8(2) \\
\hline $\mathrm{C}(31)-\mathrm{C}(36)-\mathrm{C}(37)$ & $106.9(2)$ & $\mathrm{C}(35)-\mathrm{C}(36)-\mathrm{C}(31)$ & 118.9(2) \\
\hline C(35)-C(36)-C(37) & $134.2(2)$ & C(36)-C(37)-C(38) & $129.6(2)$ \\
\hline$C(42)-C(37)-C(36)$ & $106.8(2)$ & $\mathrm{C}(42)-\mathrm{C}(37)-\mathrm{C}(38)$ & $123.5(2)$ \\
\hline C(37)-C(38)-C(39) & $111.4(2)$ & C(40)-C(39)-C(38) & $111.2(2)$ \\
\hline C(50)-C(39)-C(38) & $112.2(2)$ & $\mathrm{C}(50)-\mathrm{C}(39)-\mathrm{C}(40)$ & $110.3(2)$ \\
\hline $\mathrm{C}(41)-\mathrm{C}(40)-\mathrm{C}(39)$ & $113.3(2)$ & $\mathrm{C}(42)-\mathrm{C}(41)-\mathrm{C}(40)$ & $108.3(2)$ \\
\hline $\mathrm{N}(2)-\mathrm{C}(42)-\mathrm{C}(41)$ & $124.4(2)$ & $\mathrm{C}(37)-\mathrm{C}(42)-\mathrm{N}(2)$ & $110.2(2)$ \\
\hline$C(37)-C(42)-C(41)$ & $125.3(2)$ & $\mathrm{N}(2)-\mathrm{C}(43)-\mathrm{C}(44)$ & 113.02(19) \\
\hline$C(45)-C(44)-C(43)$ & $120.0(2)$ & $\mathrm{C}(49)-\mathrm{C}(44)-\mathrm{C}(43)$ & $121.2(2)$ \\
\hline C(49)-C(44)-C(45) & $118.8(2)$ & $\mathrm{C}(46)-\mathrm{C}(45)-\mathrm{C}(44)$ & $120.7(2)$ \\
\hline$C(45)-C(46)-C(47)$ & $119.5(2)$ & $\mathrm{C}(46)-\mathrm{C}(47)-\mathrm{I}(2)$ & 119.88(16) \\
\hline $\mathrm{C}(48)-\mathrm{C}(47)-\mathrm{I}(2)$ & 119.37(18) & $\mathrm{C}(48)-\mathrm{C}(47)-\mathrm{C}(46)$ & $120.7(2)$ \\
\hline $\mathrm{C}(47)-\mathrm{C}(48)-\mathrm{C}(49)$ & 119.3(2) & $\mathrm{C}(48)-\mathrm{C}(49)-\mathrm{C}(44)$ & $120.9(2)$ \\
\hline C(51)-C(50)-C(39) & $119.8(2)$ & $\mathrm{C}(51)-\mathrm{C}(50)-\mathrm{C}(55)$ & $118.4(2)$ \\
\hline $\mathrm{C}(55)-\mathrm{C}(50)-\mathrm{C}(39)$ & $121.7(2)$ & $\mathrm{C}(50)-\mathrm{C}(51)-\mathrm{C}(52)$ & $120.4(3)$ \\
\hline C(53)-C(52)-C(51) & 121.2(3) & $\mathrm{C}(52)-\mathrm{C}(53)-\mathrm{C}(54)$ & 119.2(2) \\
\hline C(53)-C(54)-C(55) & 119.9(3) & $\mathrm{C}(54)-\mathrm{C}(55)-\mathrm{C}(50)$ & 120.9(3) \\
\hline
\end{tabular}


Table 4. Anisotropic displacement parameters $\left(\AA^{2}\right)$.

The anisotropic displacement factor exponent takes the form:

$-2 \pi^{2}\left[h^{2} a^{* 2} U_{11}+\ldots+2 h k\right.$ a* b* $\left.U_{12}\right]$.

\begin{tabular}{|c|c|c|c|c|c|}
\hline $\mathrm{U}_{11}$ & $\mathrm{U}_{22}$ & $\mathrm{U}_{33}$ & $\mathrm{U}_{23}$ & $\mathrm{U}_{13}$ & $\mathrm{U}$ \\
\hline $\mathrm{I}(1) \quad 0.027(1)$ & $0.036(1)$ & $0.017(1)$ & $-0.002(1)$ & $-0.007(1)$ & $-0.005(1)$ \\
\hline $\mathrm{I}(2) \quad 0.025(1)$ & $0.033(1)$ & $0.017(1)$ & $0.003(1)$ & $-0.006(1)$ & $0.003(1)$ \\
\hline $\mathrm{N}(1) 0.022(1)$ & $0.015(1)$ & $0.019(1)$ & $0.000(1)$ & $-0.007(1)$ & $0.003(1)$ \\
\hline $\mathrm{N}(2) 0.018(1)$ & $0.015(1)$ & $0.018(1)$ & $-0.001(1)$ & $-0.004(1)$ & $-0.001(1)$ \\
\hline $\mathrm{C}(1) 0.016(1)$ & $0.016(1)$ & $0.022(1)$ & $0.000(1)$ & $-0.005(1)$ & $-0.001(1)$ \\
\hline $\mathrm{C}(2) 0.018(1)$ & $0.023(1)$ & $0.022(1)$ & $0.001(1)$ & $-0.003(1)$ & $-0.005(1)$ \\
\hline $\mathrm{C}(3) 0.017(1)$ & $0.027(1)$ & $0.025(1)$ & $-0.005(1)$ & $0.001(1)$ & $0.000(1)$ \\
\hline $\mathrm{C}(4) 0.018(1)$ & $0.024(1)$ & $0.029(1)$ & $-0.003(1)$ & $-0.004(1)$ & $0.004(1)$ \\
\hline $\mathrm{C}(5) 0.018(1)$ & $0.019(1)$ & $0.024(1)$ & $0.000(1)$ & $-0.006(1)$ & $0.005(1)$ \\
\hline$C(6) 0.014(1)$ & $0.015(1)$ & $0.022(1)$ & $-0.001(1)$ & $-0.006(1)$ & $-0.002(1)$ \\
\hline $\mathrm{C}(7) 0.017(1)$ & $0.014(1)$ & $0.021(1)$ & $-0.001(1)$ & $-0.006(1)$ & $0.001(1)$ \\
\hline $\mathrm{C}(8) 0.019(1)$ & $0.018(1)$ & $0.020(1)$ & $-0.002(1)$ & $-0.003(1)$ & $0.000(1)$ \\
\hline $\mathrm{C}(9) 0.020(1)$ & $0.014(1)$ & $0.021(1)$ & $-0.002(1)$ & $-0.001(1)$ & $0.000(1)$ \\
\hline $\mathrm{C}(10) 0.022(1)$ & $0.026(1)$ & $0.028(1)$ & $-0.006(1)$ & $-0.005(1)$ & $0.011(1)$ \\
\hline $\mathrm{C}(11) 0.028(2)$ & $0.018(1)$ & $0.027(1)$ & $-0.002(1)$ & $-0.009(1)$ & $0.009(1)$ \\
\hline $\mathrm{C}(12) 0.021(1)$ & $0.016(1)$ & $0.021(1)$ & & & $0.002(1)$ \\
\hline $\mathrm{C}(13) 0.024(1)$ & $0.017(1)$ & $0.024(1)$ & $0.003(1)$ & $7(1)$ & $0.000(1)$ \\
\hline $\mathrm{C}(14) 0.019(1)$ & $0.015(1)$ & $0.019(1)$ & $0.001(1)$ & $-0.005(1)$ & $0.006(1)$ \\
\hline $\mathrm{C}(15) 0.023(1)$ & $0.019(1)$ & $0.022(1)$ & $0.004(1)$ & $-0.002(1)$ & $-0.001(1)$ \\
\hline $\mathrm{C}(16) 0.025(1)$ & $0.027(1)$ & $0.014(1)$ & $0.004(1)$ & $-0.002(1)$ & $0.002(1)$ \\
\hline C(17)0.019(1) & $0.019(1)$ & $0.017(1)$ & $-0.002(1)$ & $-0.004(1)$ & $0.003(1)$ \\
\hline $\mathrm{C}(18) 0.019(1)$ & $0.017(1)$ & $0.017(1)$ & $0.001(1)$ & $-0.002(1)$ & $0.002(1)$ \\
\hline C(19)0.022(1) & $0.017(1)$ & $0.014(1)$ & $0.000(1)$ & $-0.003(1)$ & $0.003(1)$ \\
\hline $\mathrm{C}(20) 0.019(1)$ & $0.014(1)$ & $0.025(1)$ & $-0.002(1)$ & $0.000(1)$ & $0.003(1)$ \\
\hline C(21)0.022(1) & $0.020(1)$ & $0.024(1)$ & $-0.003(1)$ & $0.002(1)$ & $-0.004(1)$ \\
\hline $\mathrm{C}(22) 0.028(1)$ & $0.023(1)$ & $0.022(1)$ & $-0.004(1)$ & $-0.001(1)$ & $-0.004(1)$ \\
\hline $\mathrm{C}(23) 0.031(2)$ & $0.020(1)$ & $0.024(1)$ & $-0.001(1)$ & $0.006(1)$ & $0.001(1)$ \\
\hline $\mathrm{C}(24) 0.018(1)$ & $0.018(1)$ & $0.038(1)$ & $0.002(1)$ & $0.003(1)$ & $0.000(1)$ \\
\hline $\mathrm{C}(25) 0.019(1)$ & $0.017(1)$ & $0.032(1)$ & $0.000(1)$ & $-0.005(1)$ & $-0.001(1)$ \\
\hline C(31)0.015(1) & $0.014(1)$ & $0.018(1)$ & $0.002(1)$ & $-0.004(1)$ & $0.002(1)$ \\
\hline C(32)0.021(1) & $0.022(1)$ & $0.017(1)$ & $0.000(1)$ & $-0.001(1)$ & $0.003(1)$ \\
\hline C(33)0.021(1) & $0.029(1)$ & $0.024(1)$ & $0.006(1)$ & $0.001(1)$ & $0.002(1)$ \\
\hline C(34)0.019(1) & $0.025(1)$ & $0.025(1)$ & $0.004(1)$ & $-0.001(1)$ & $-0.007(1)$ \\
\hline
\end{tabular}




\begin{tabular}{lllrrr}
$\mathrm{C}(35) 0.020(1)$ & $0.017(1)$ & $0.021(1)$ & $0.001(1)$ & $-0.005(1)$ & $-0.001(1)$ \\
$\mathrm{C}(36) 0.018(1)$ & $0.014(1)$ & $0.018(1)$ & $0.001(1)$ & $-0.003(1)$ & $0.001(1)$ \\
$\mathrm{C}(37) 0.017(1)$ & $0.015(1)$ & $0.017(1)$ & $0.002(1)$ & $-0.003(1)$ & $-0.001(1)$ \\
$\mathrm{C}(38) 0.021(1)$ & $0.013(1)$ & $0.017(1)$ & $0.000(1)$ & $-0.002(1)$ & $-0.004(1)$ \\
$\mathrm{C}(39) 0.020(1)$ & $0.021(1)$ & $0.021(1)$ & $0.003(1)$ & $0.000(1)$ & $0.000(1)$ \\
$\mathrm{C}(40) 0.023(1)$ & $0.023(1)$ & $0.024(1)$ & $0.004(1)$ & $-0.001(1)$ & $-0.004(1)$ \\
$\mathrm{C}(41) 0.027(2)$ & $0.018(1)$ & $0.021(1)$ & $0.001(1)$ & $-0.004(1)$ & $-0.008(1)$ \\
$\mathrm{C}(42) 0.018(1)$ & $0.013(1)$ & $0.020(1)$ & $0.002(1)$ & $-0.004(1)$ & $0.000(1)$ \\
$\mathrm{C}(43) 0.025(1)$ & $0.014(1)$ & $0.018(1)$ & $-0.004(1)$ & $-0.007(1)$ & $0.001(1)$ \\
$\mathrm{C}(44) 0.014(1)$ & $0.014(1)$ & $0.018(1)$ & $-0.002(1)$ & $-0.002(1)$ & $0.000(1)$ \\
$\mathrm{C}(45) 0.022(1)$ & $0.022(1)$ & $0.018(1)$ & $-0.005(1)$ & $0.000(1)$ & $0.000(1)$ \\
$\mathrm{C}(46) 0.025(1)$ & $0.025(1)$ & $0.012(1)$ & $-0.002(1)$ & $-0.002(1)$ & $-0.002(1)$ \\
$\mathrm{C}(47) 0.017(1)$ & $0.023(1)$ & $0.014(1)$ & $0.002(1)$ & $-0.002(1)$ & $-0.003(1)$ \\
$\mathrm{C}(48) 0.021(1)$ & $0.017(1)$ & $0.014(1)$ & $0.000(1)$ & $-0.001(1)$ & $0.001(1)$ \\
$\mathrm{C}(49) 0.020(1)$ & $0.015(1)$ & $0.013(1)$ & $-0.002(1)$ & $-0.003(1)$ & $0.000(1)$ \\
$\mathrm{C}(50) 0.021(1)$ & $0.016(1)$ & $0.021(1)$ & $0.001(1)$ & $0.000(1)$ & $-0.004(1)$ \\
$\mathrm{C}(51) 0.020(1)$ & $0.017(1)$ & $0.024(1)$ & $-0.001(1)$ & $-0.004(1)$ & $-0.002(1)$ \\
$\mathrm{C}(52) 0.022(1)$ & $0.022(1)$ & $0.024(1)$ & $-0.003(1)$ & $0.001(1)$ & $-0.003(1)$ \\
$\mathrm{C}(53) 0.027(1)$ & $0.023(1)$ & $0.019(1)$ & $0.002(1)$ & $0.003(1)$ & $-0.001(1)$ \\
$\mathrm{C}(54) 0.025(1)$ & $0.025(1)$ & $0.027(1)$ & $0.004(1)$ & $0.001(1)$ & $0.007(1)$ \\
$\mathrm{C}(55) 0.021(1)$ & $0.024(1)$ & $0.026(1)$ & $0.001(1)$ & $0.004(1)$ & $0.003(1)$ \\
& & & & & \\
\hline
\end{tabular}




\section{Copies of NMR spectra}

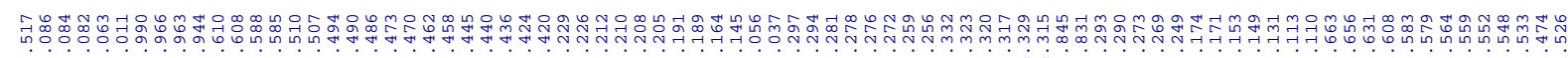

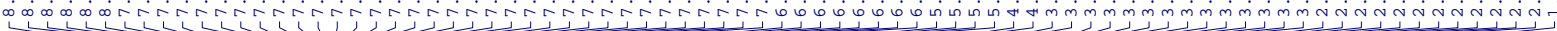

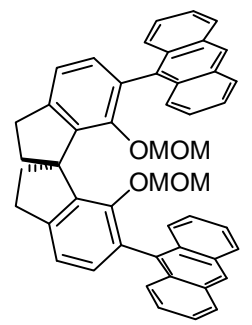

$(R)-\mathrm{C}$

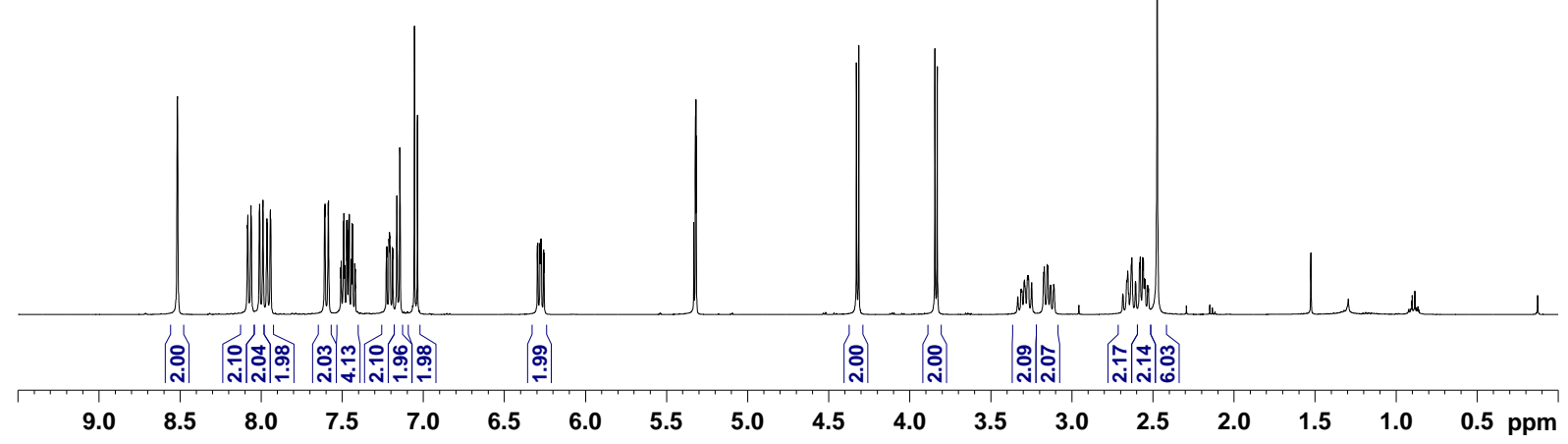

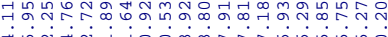

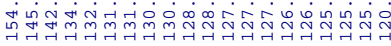

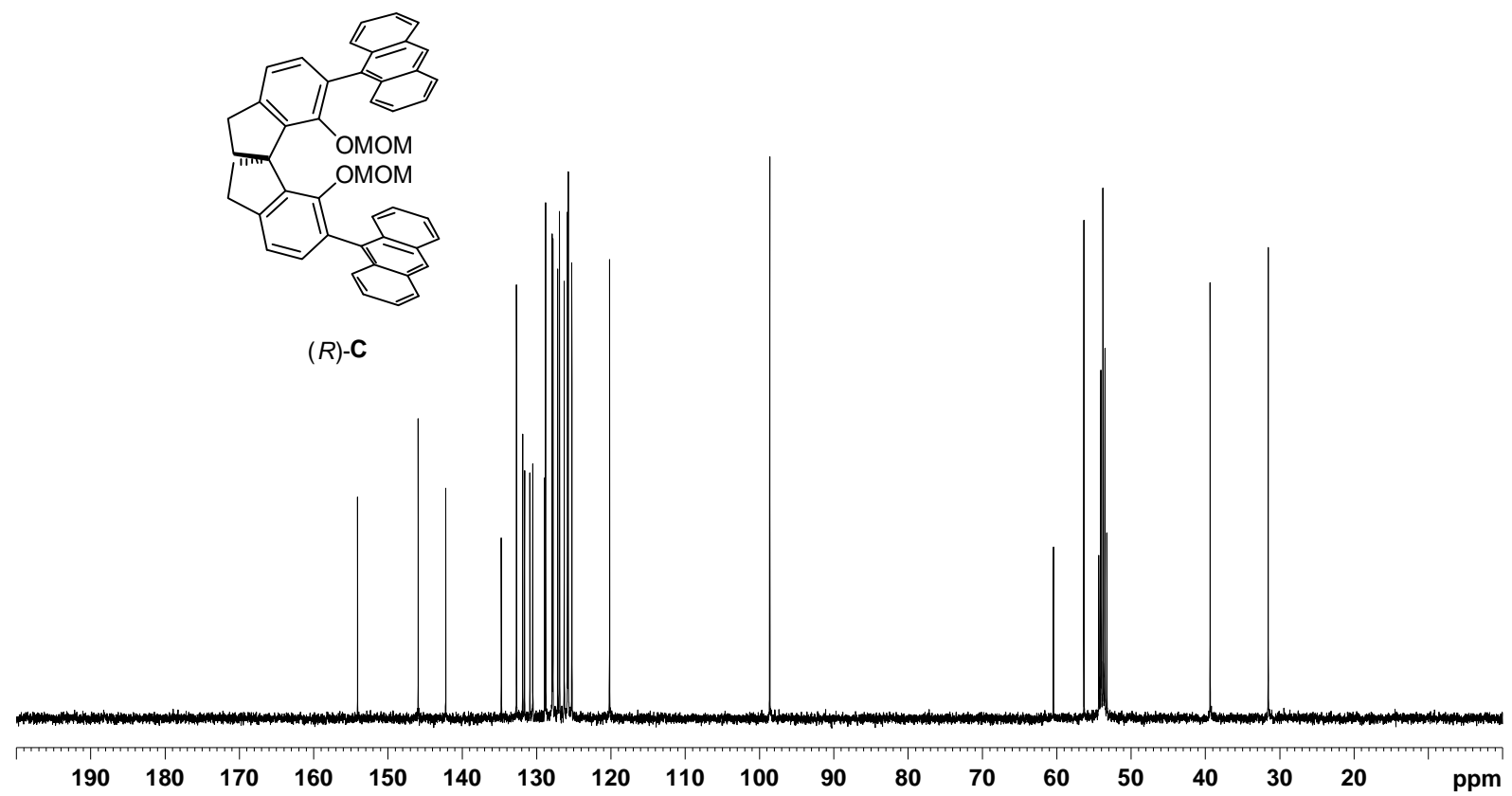




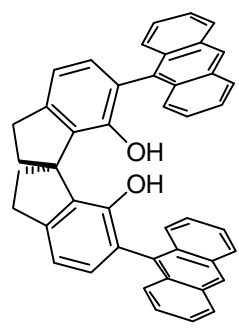

$(R)-\mathrm{D}$
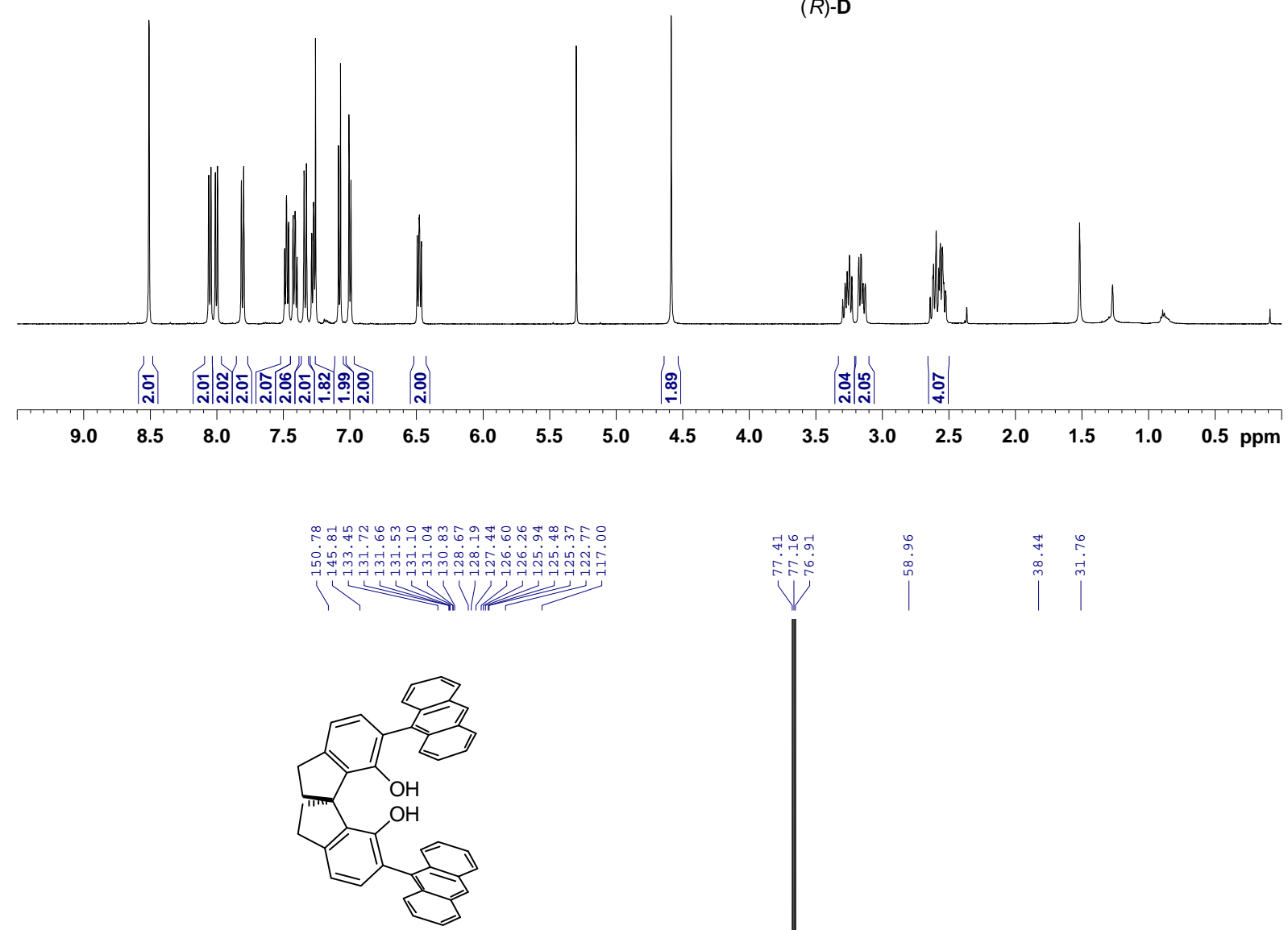

$(R)-\mathrm{D}$

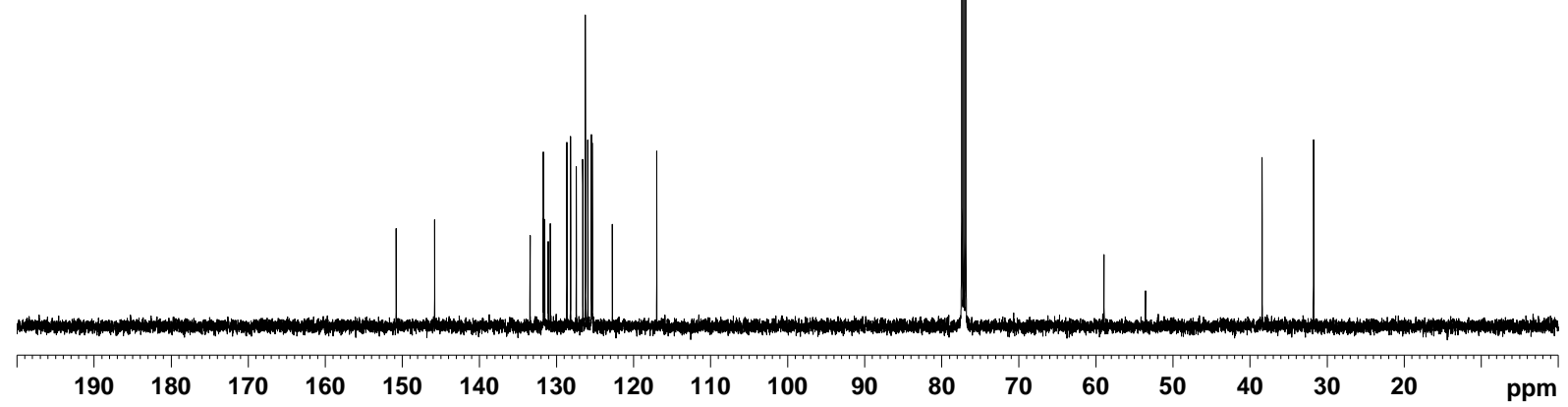




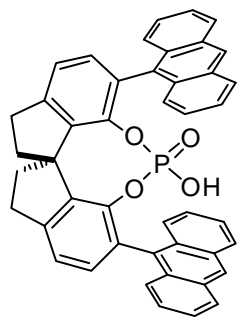

$(R)-5 f$
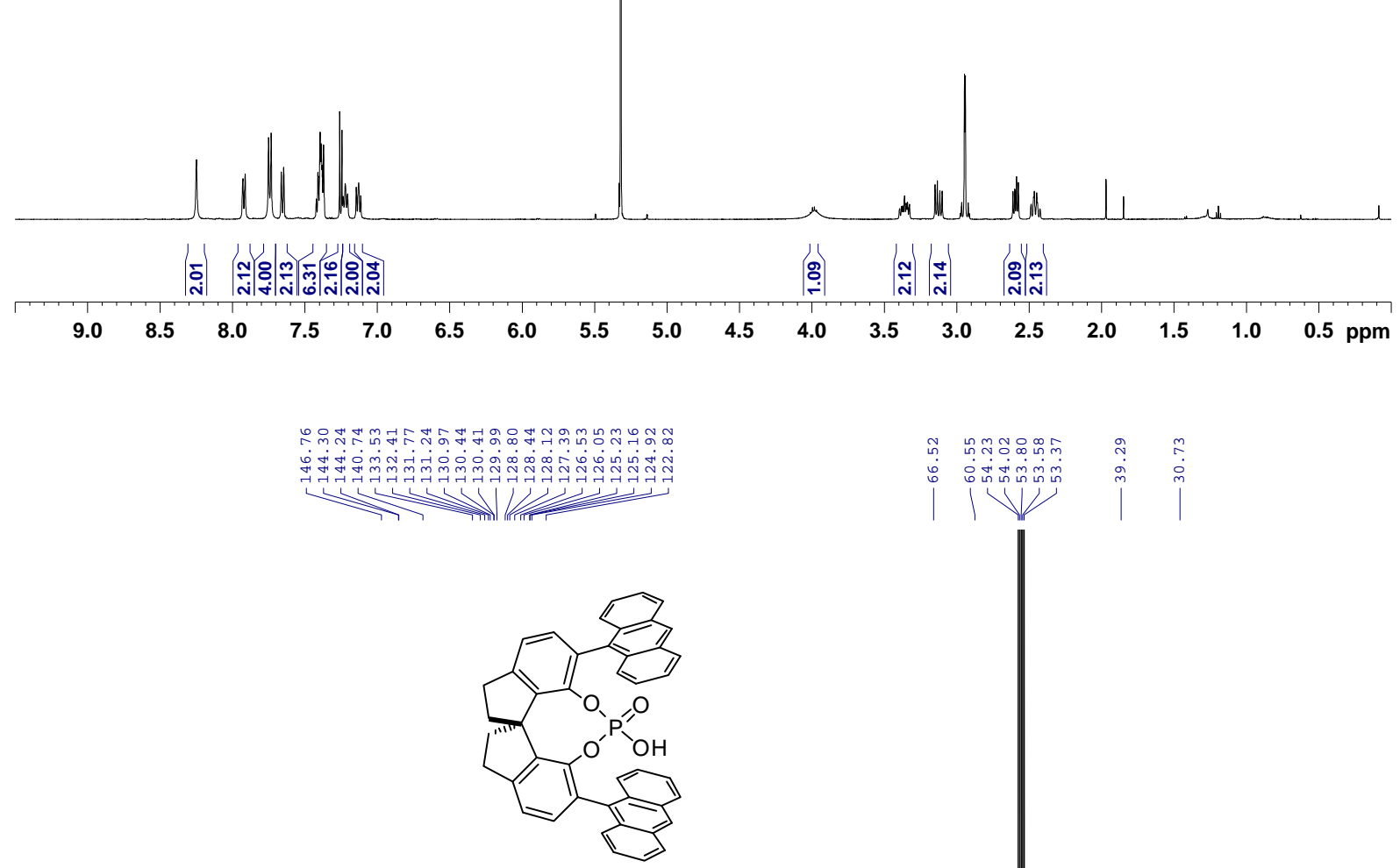

$(R)-5 f$

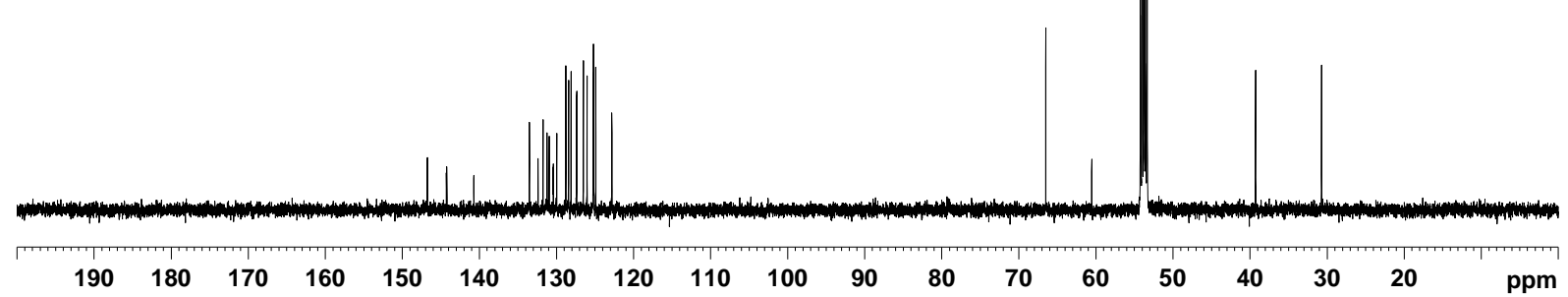




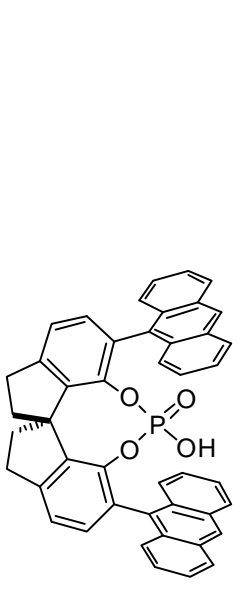

(R)-5f

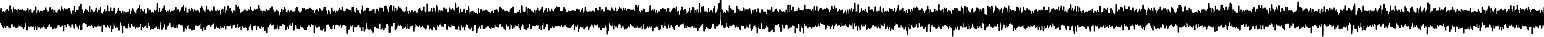



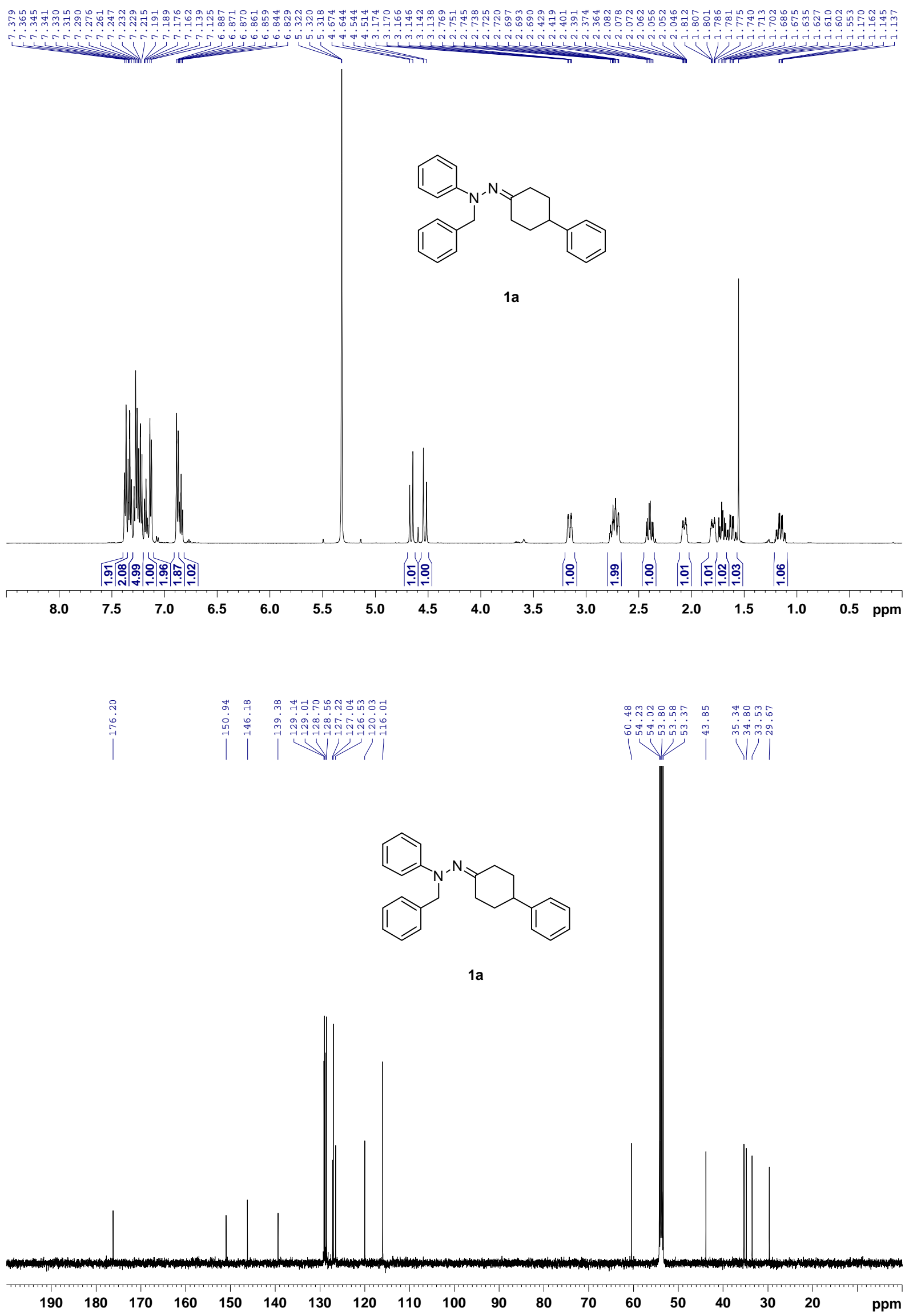

S64 


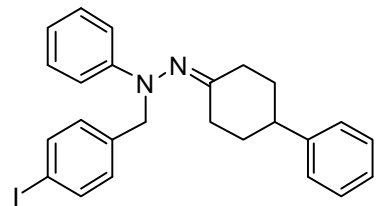

$1 \mathrm{~b}$

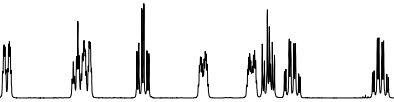

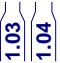

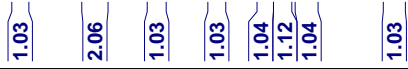

:

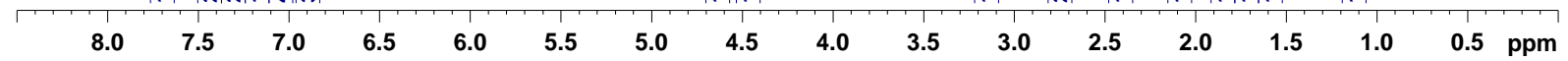
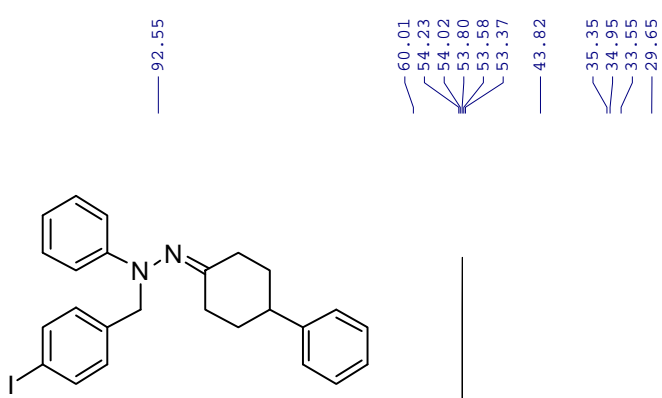

$1 b$

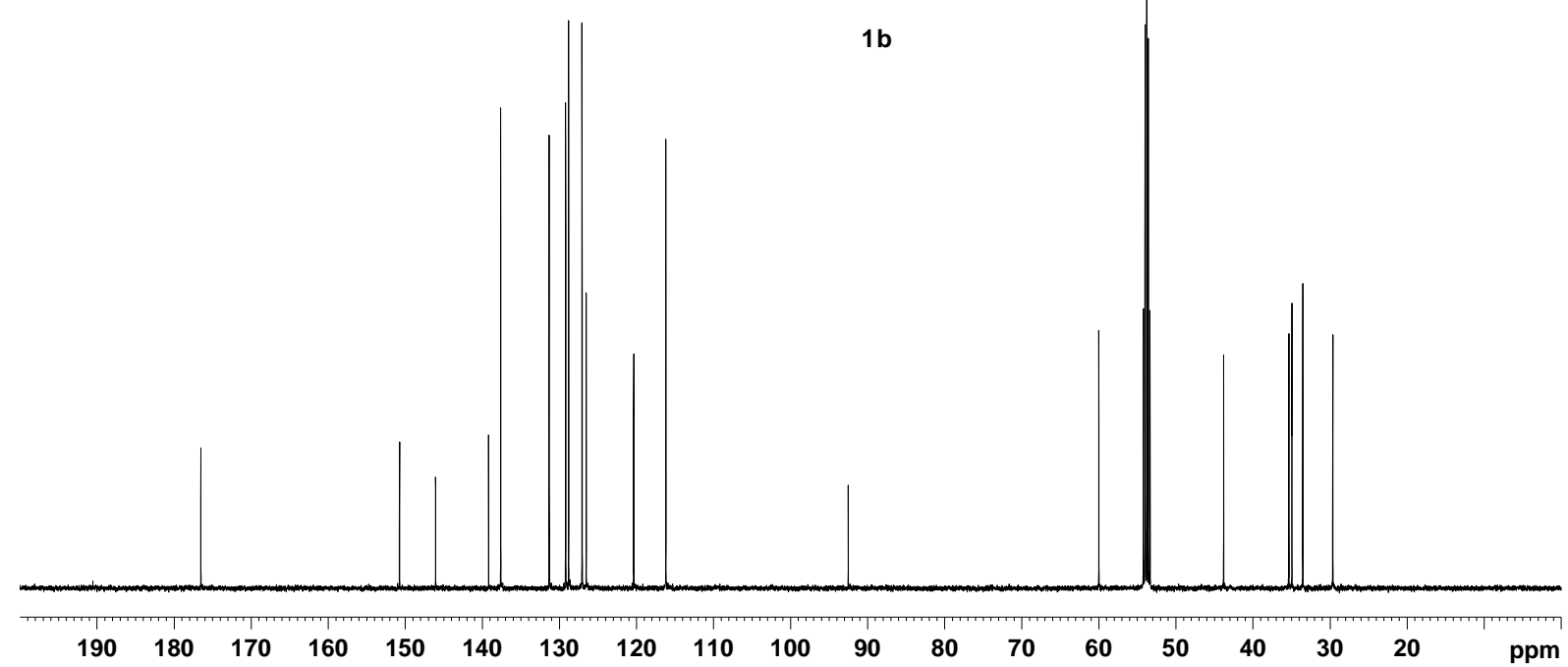




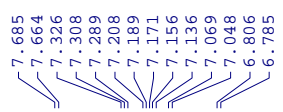

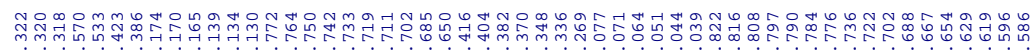

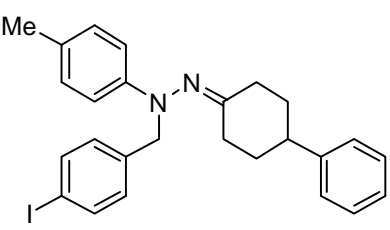

$1 c$

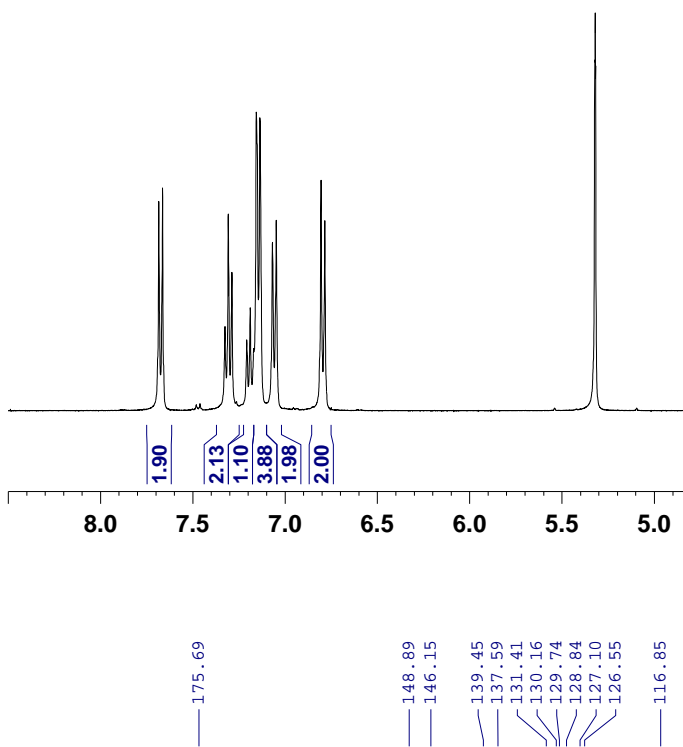

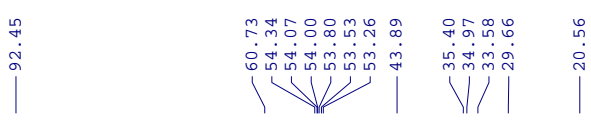

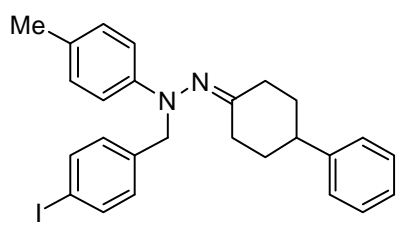

$1 c$

S66 


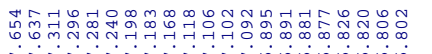
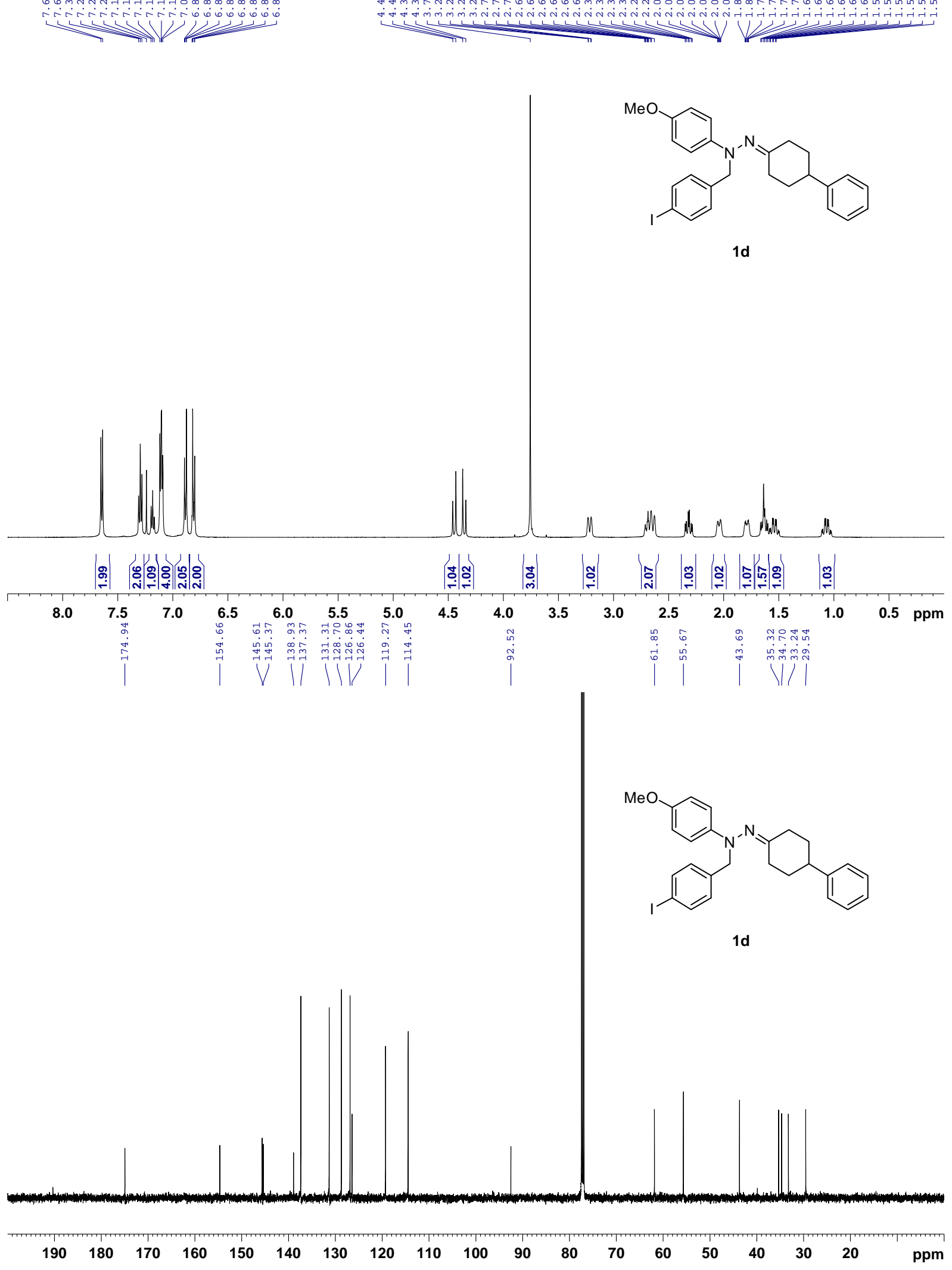

S67 

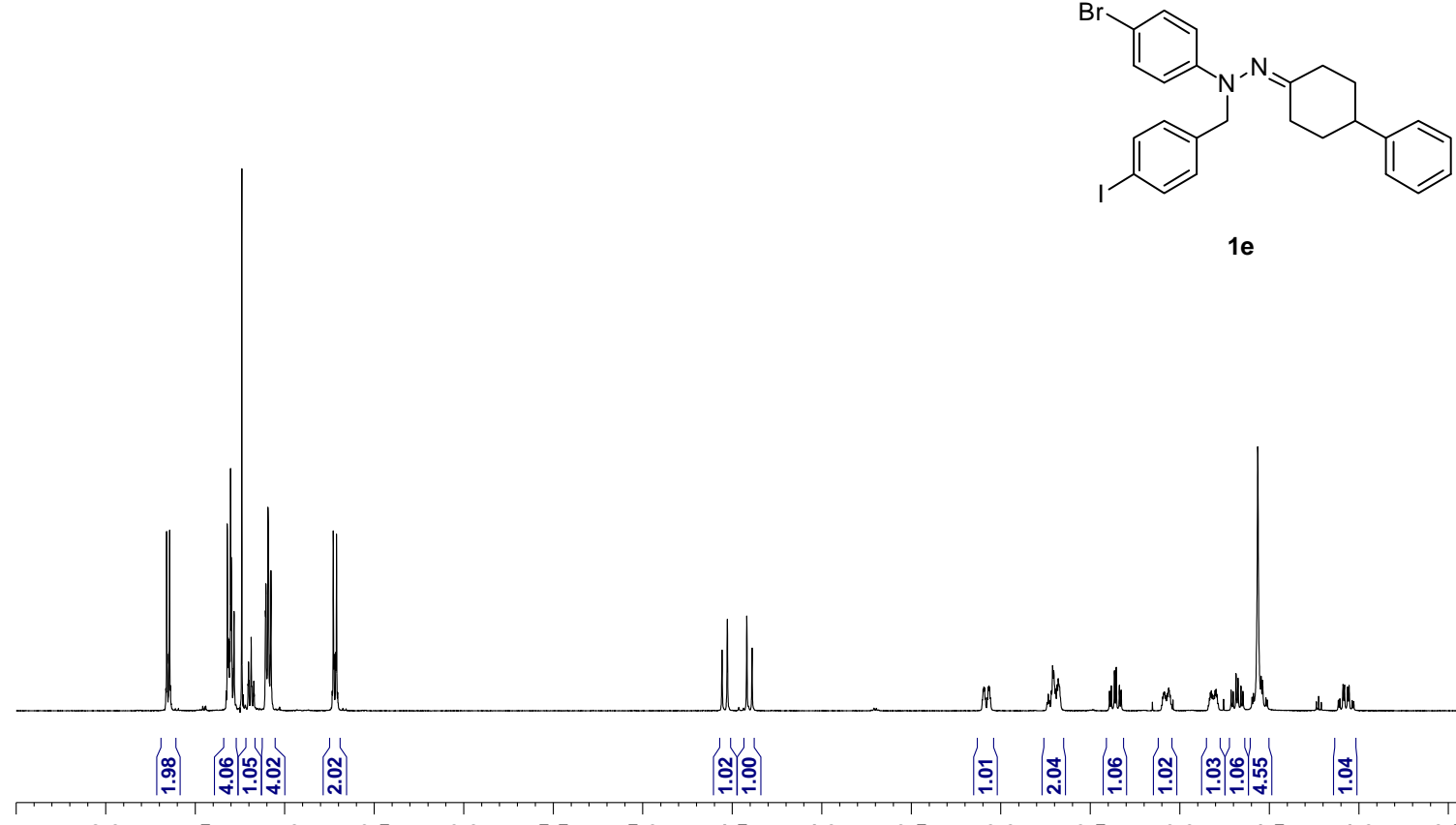

$1 e$

:

8.0

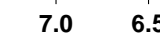

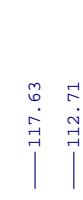

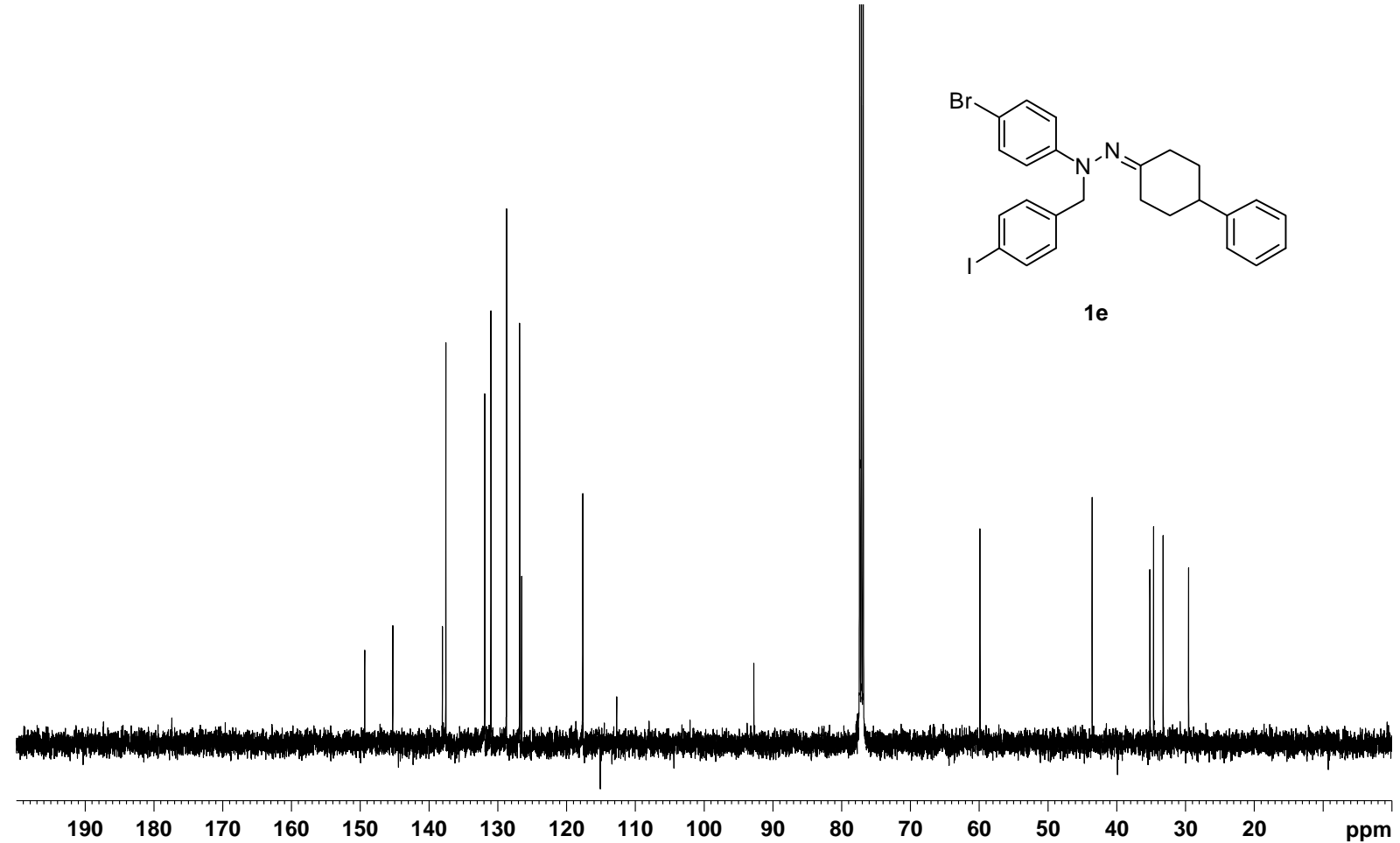




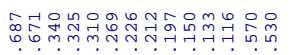

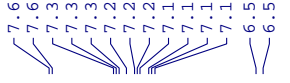

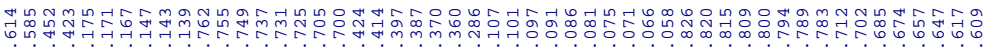

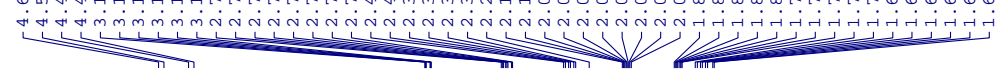

$1 f$
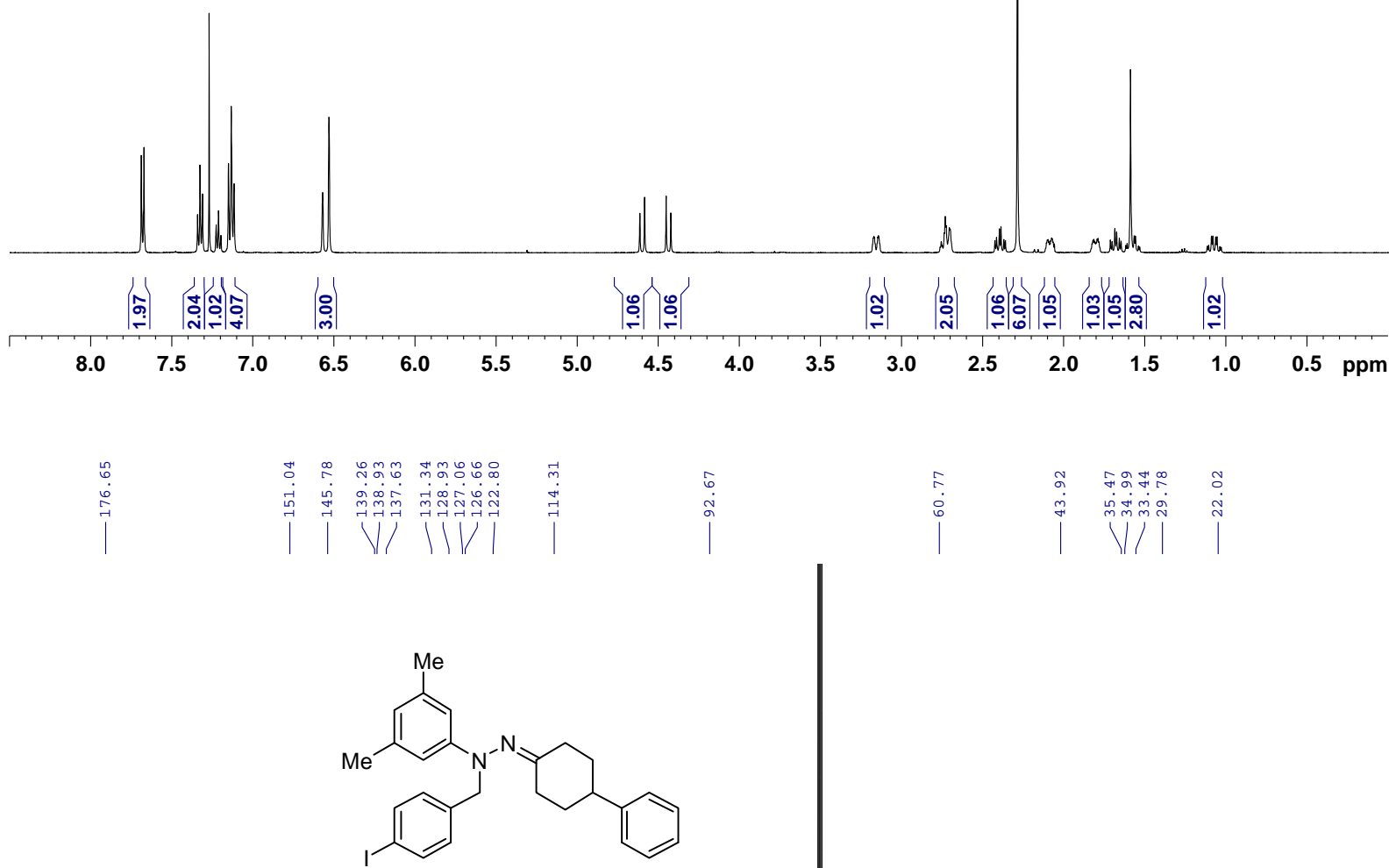

$1 f$

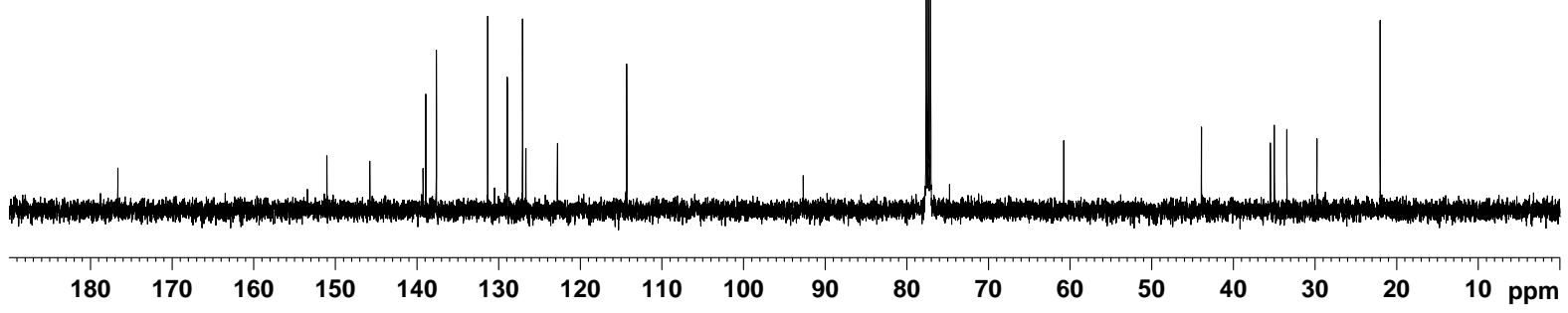

S69 


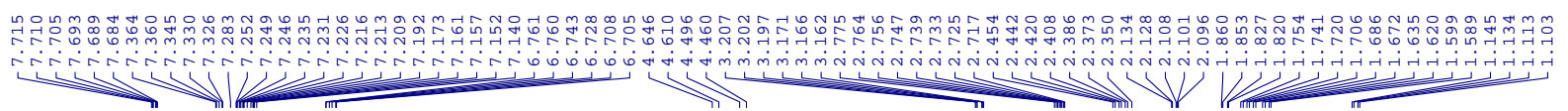

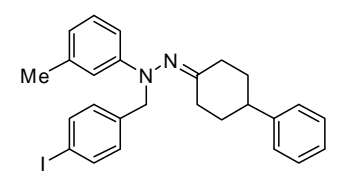

$1 \mathrm{~g}$
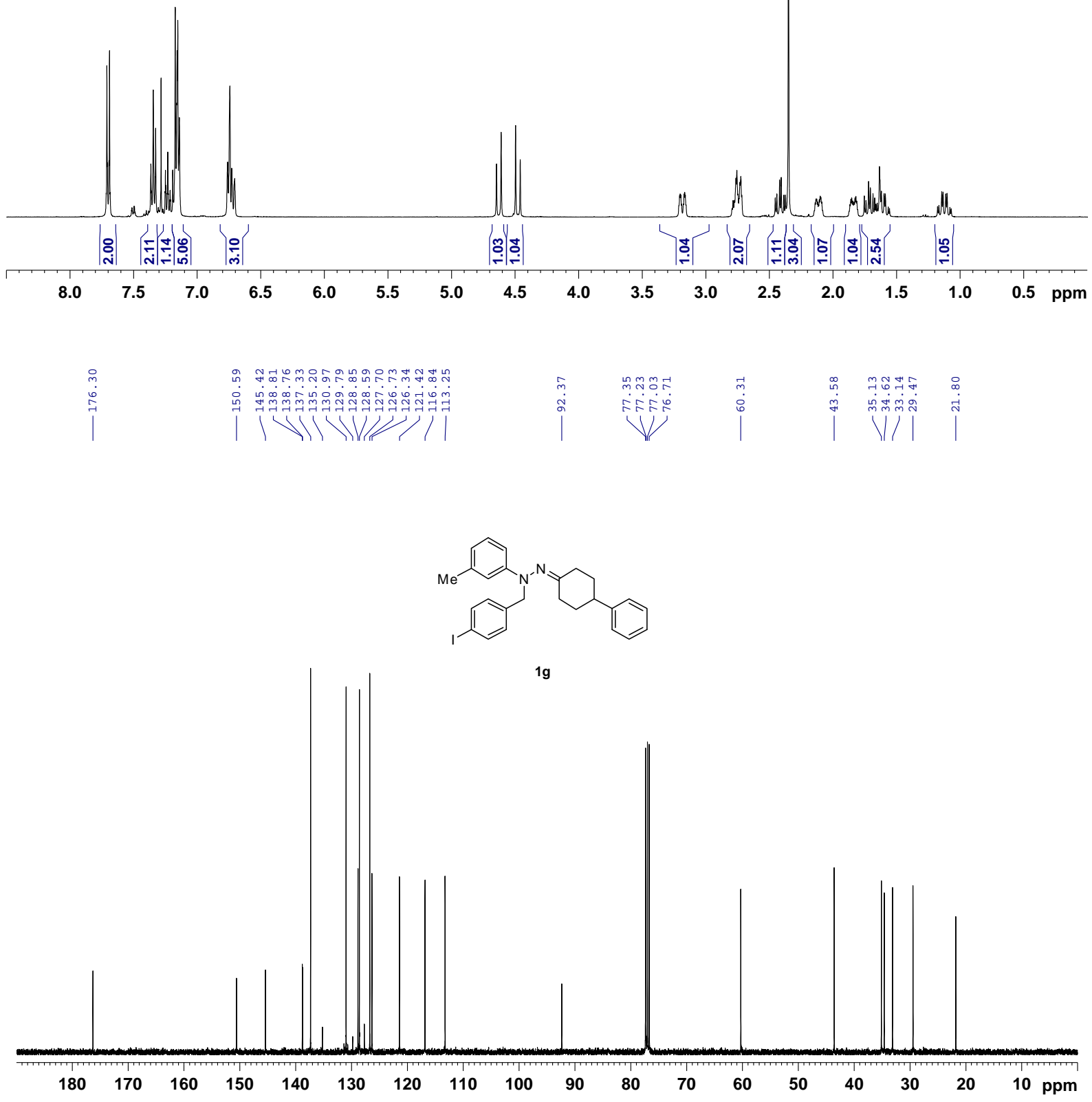

S70 


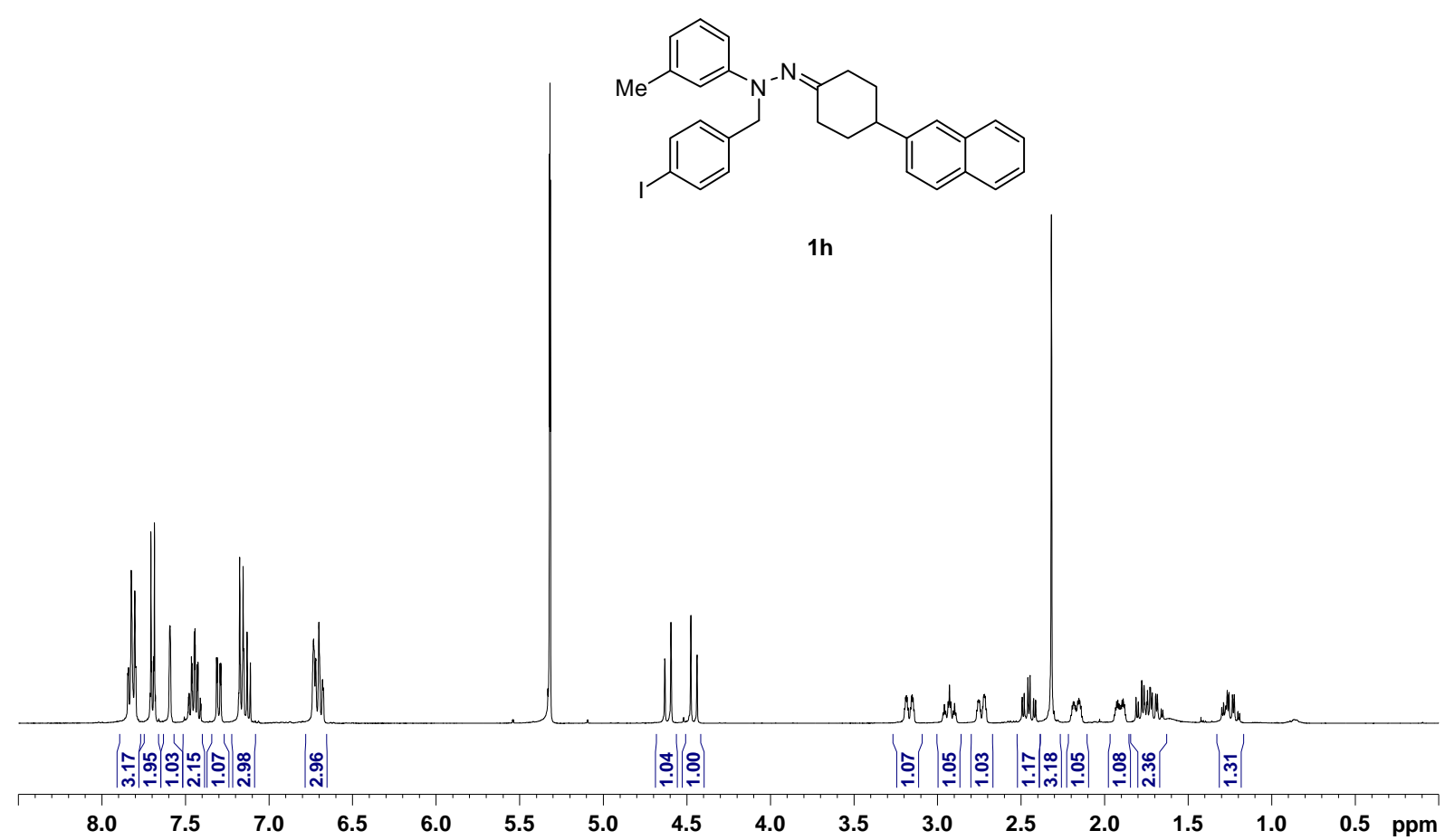

\begin{tabular}{|c|c|c|c|c|}
\hline$\stackrel{\infty}{\rightarrow}$ & 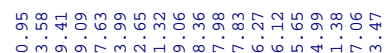 & $\stackrel{\varphi}{q}$ & 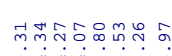 & \\
\hline & 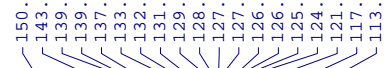 & ๙ั่ & 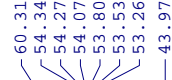 & क्षे \\
\hline
\end{tabular}

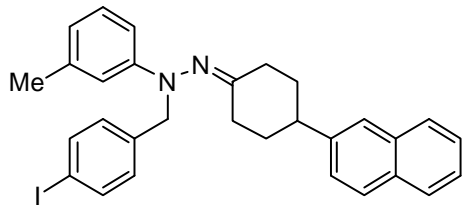

1h

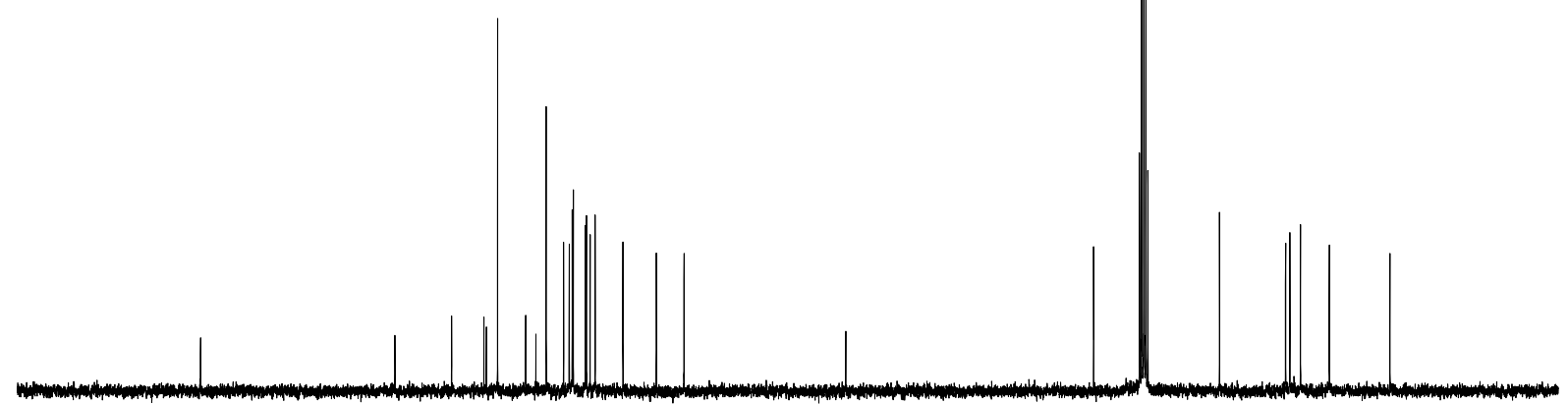

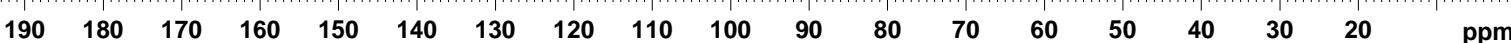




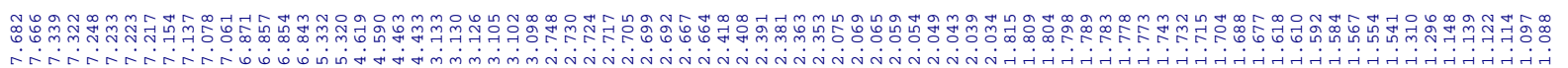

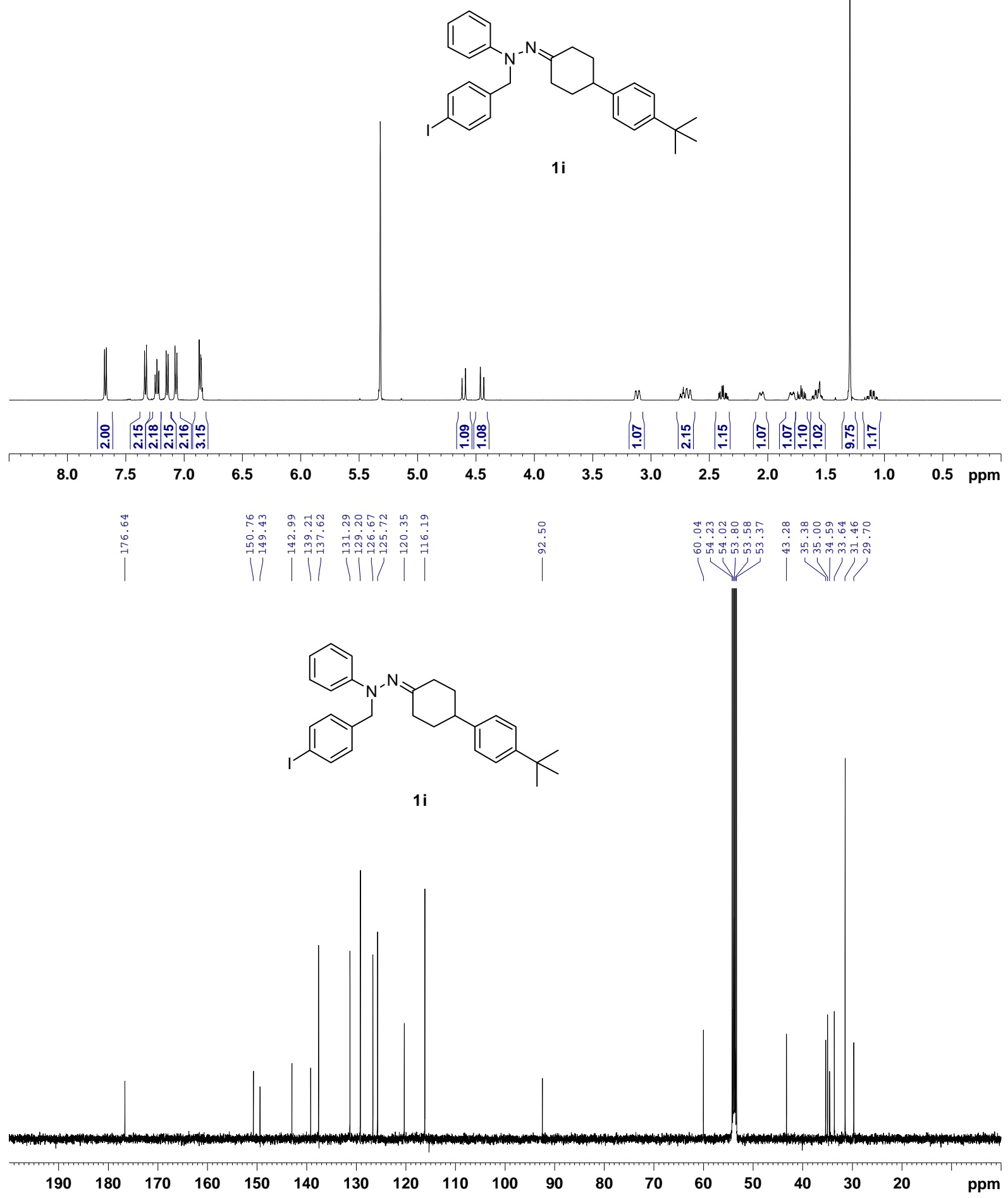

S72 


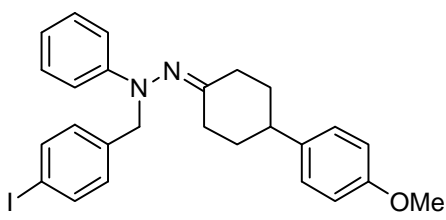

$1 \mathrm{j}$

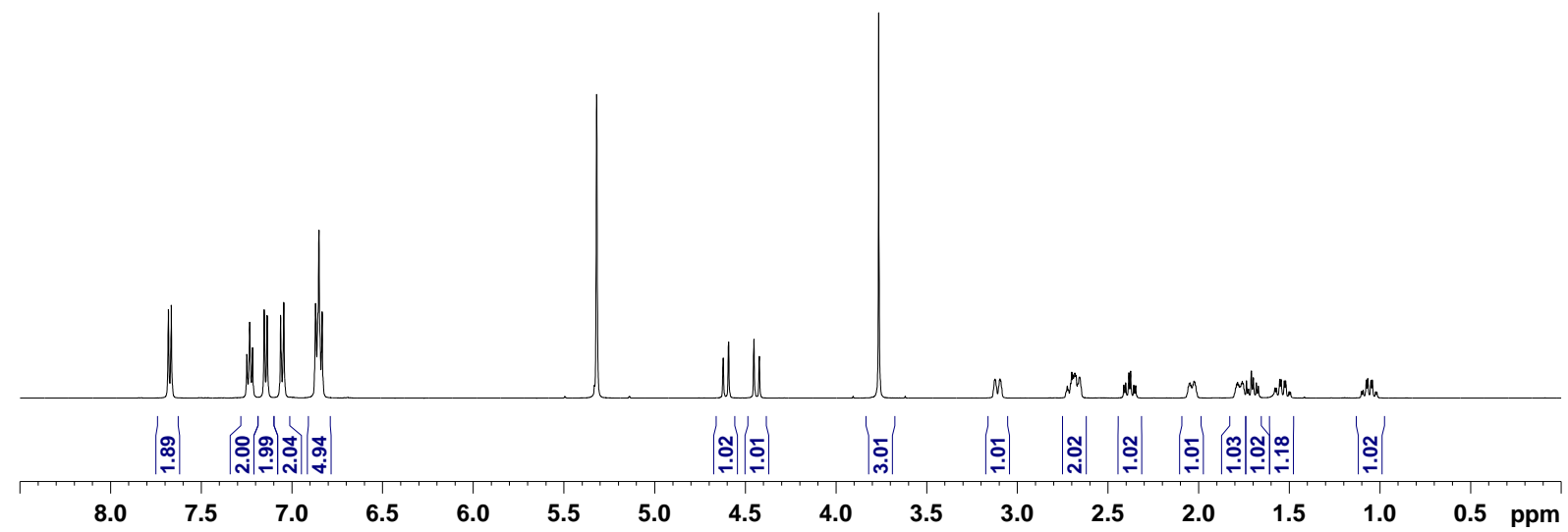

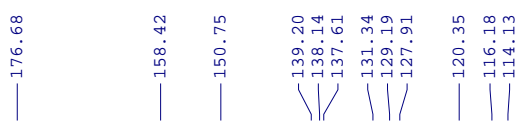

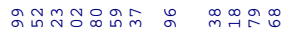

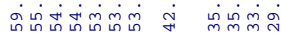<smiles>COc1ccc(C2CCC(=NN(Cc3ccc(I)cc3)c3ccccc3)CC2)cc1</smiles>

$1 \mathrm{j}$

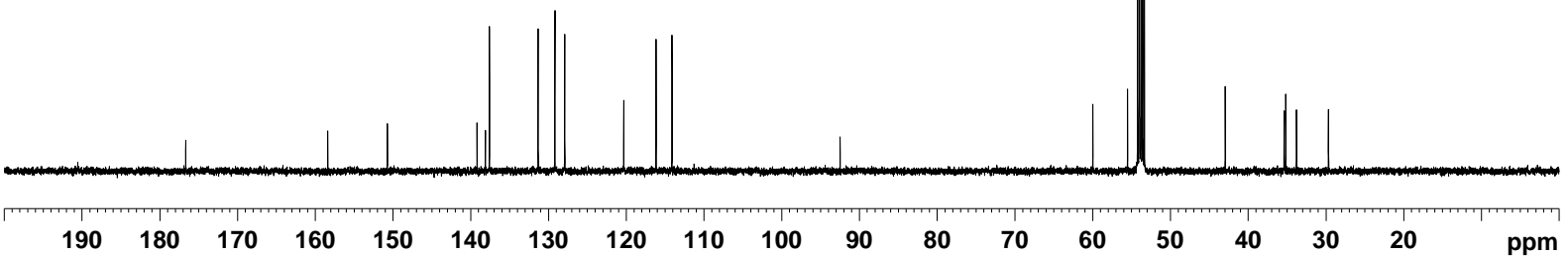




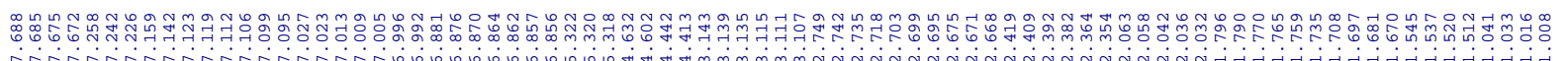

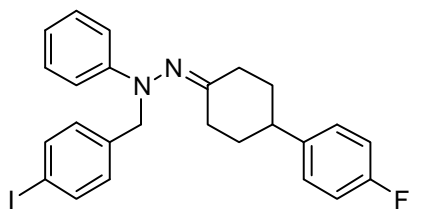

$1 \mathrm{k}$
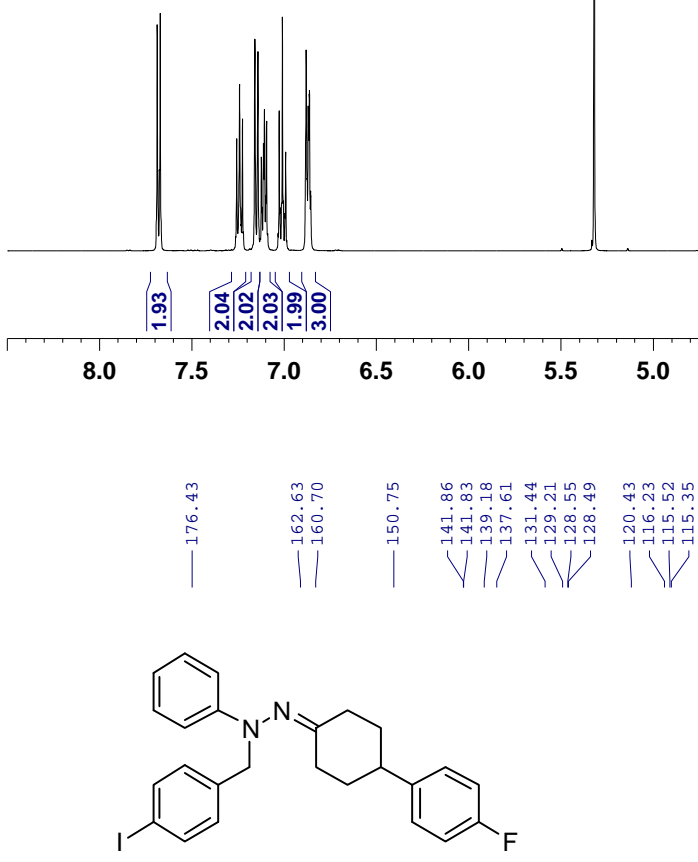

$1 \mathrm{k}$

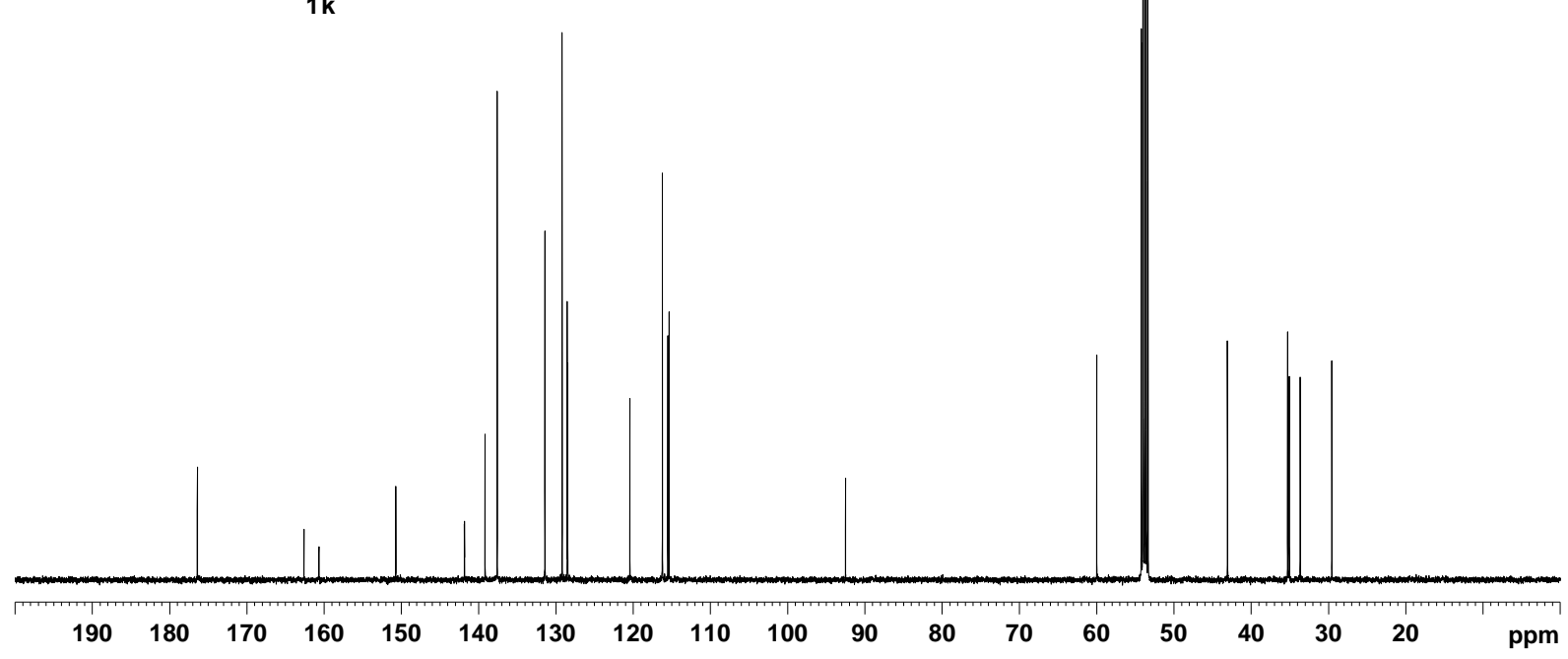

S74 


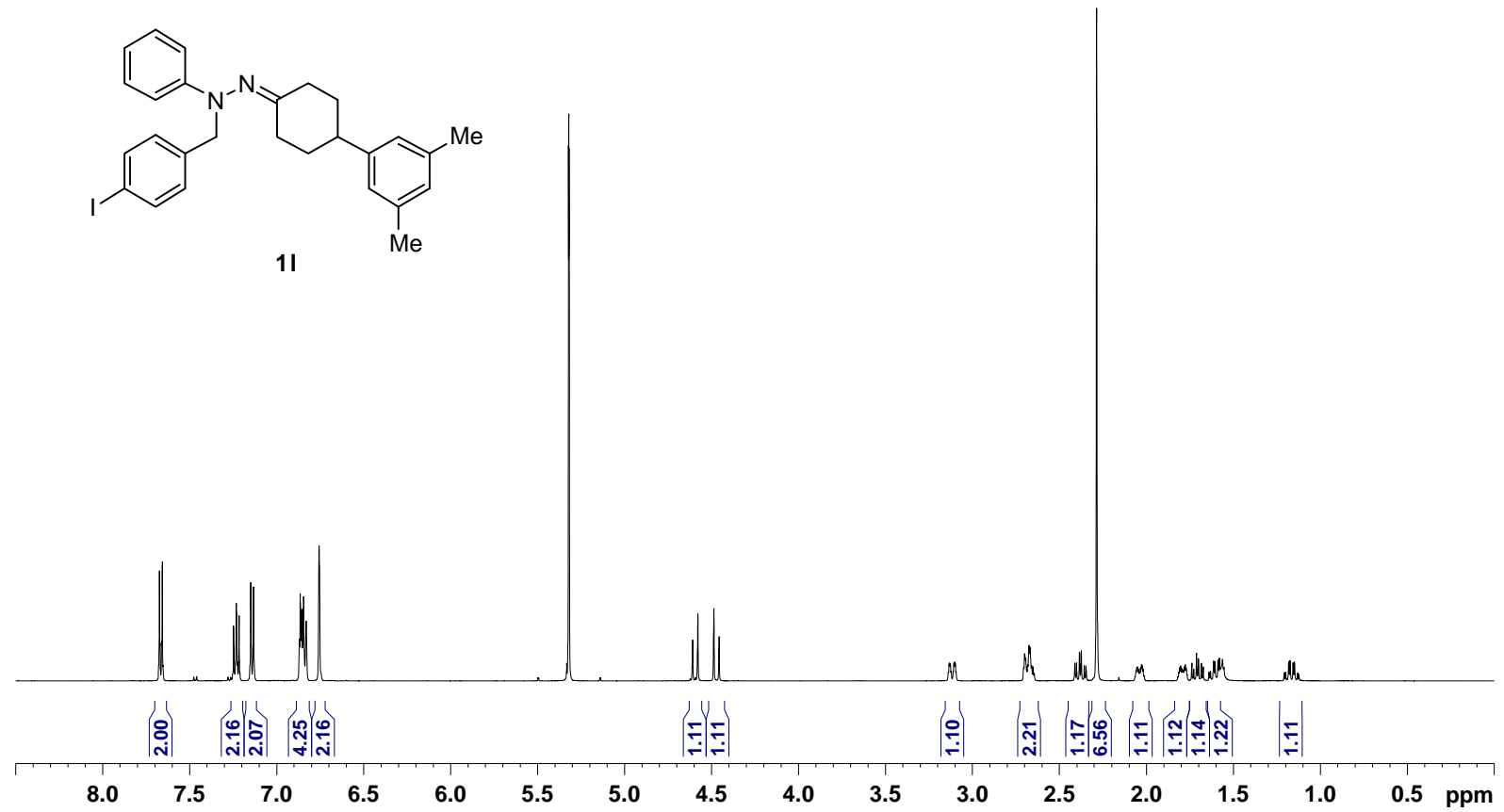

ભ<smiles>Cc1cc(C)cc(C2CCC(=NN(Cc3ccc(I)cc3)c3ccccc3)CC2)c1</smiles>

11
Hก⿻心

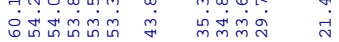
IW I/I I 告 告

$|/||l| \mid$

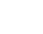

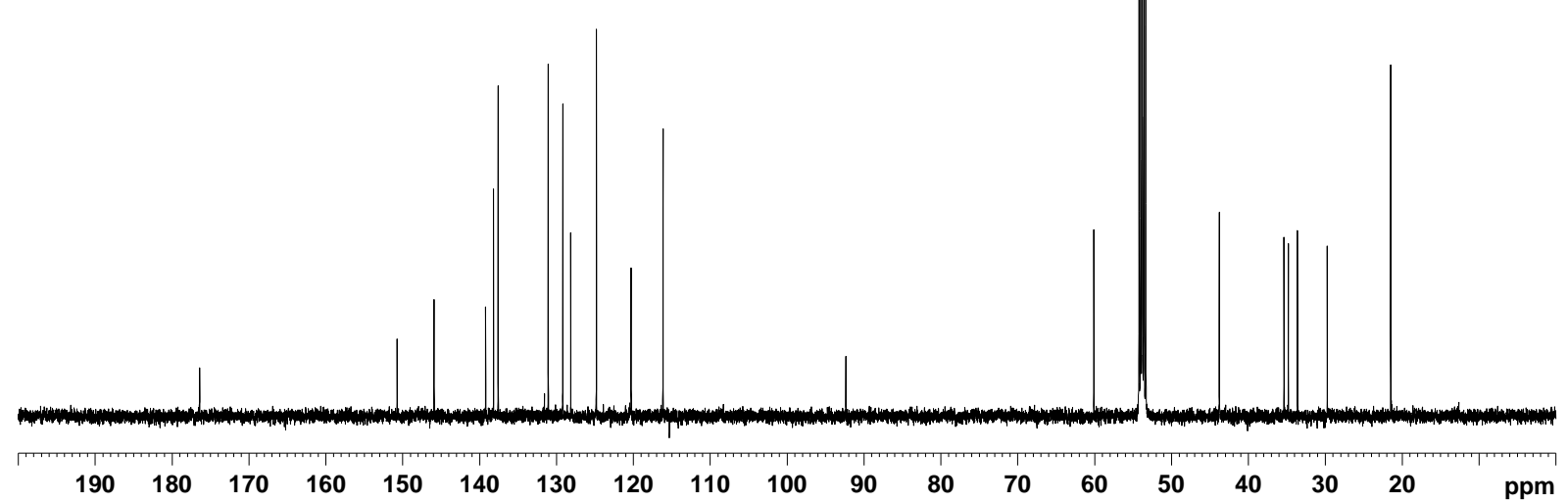




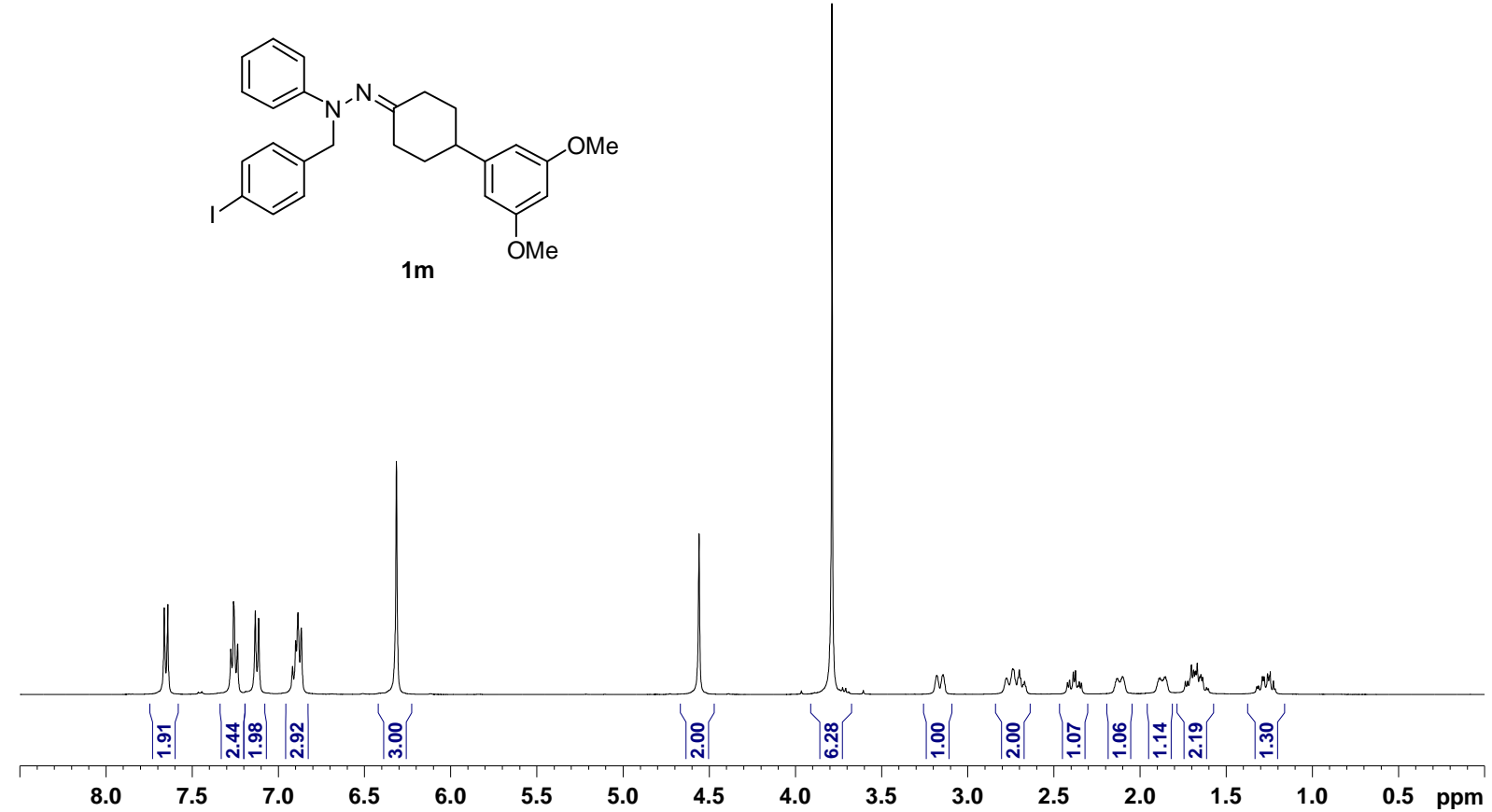

$1 \mathrm{~m}$

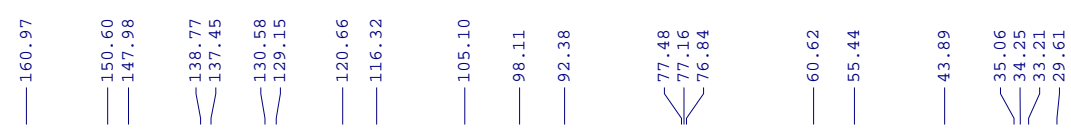<smiles>COc1cc(OC)cc(C2CCC(=NN(Cc3ccccc3)c3ccc(I)cc3)CC2)c1</smiles>

$1 \mathrm{~m}$
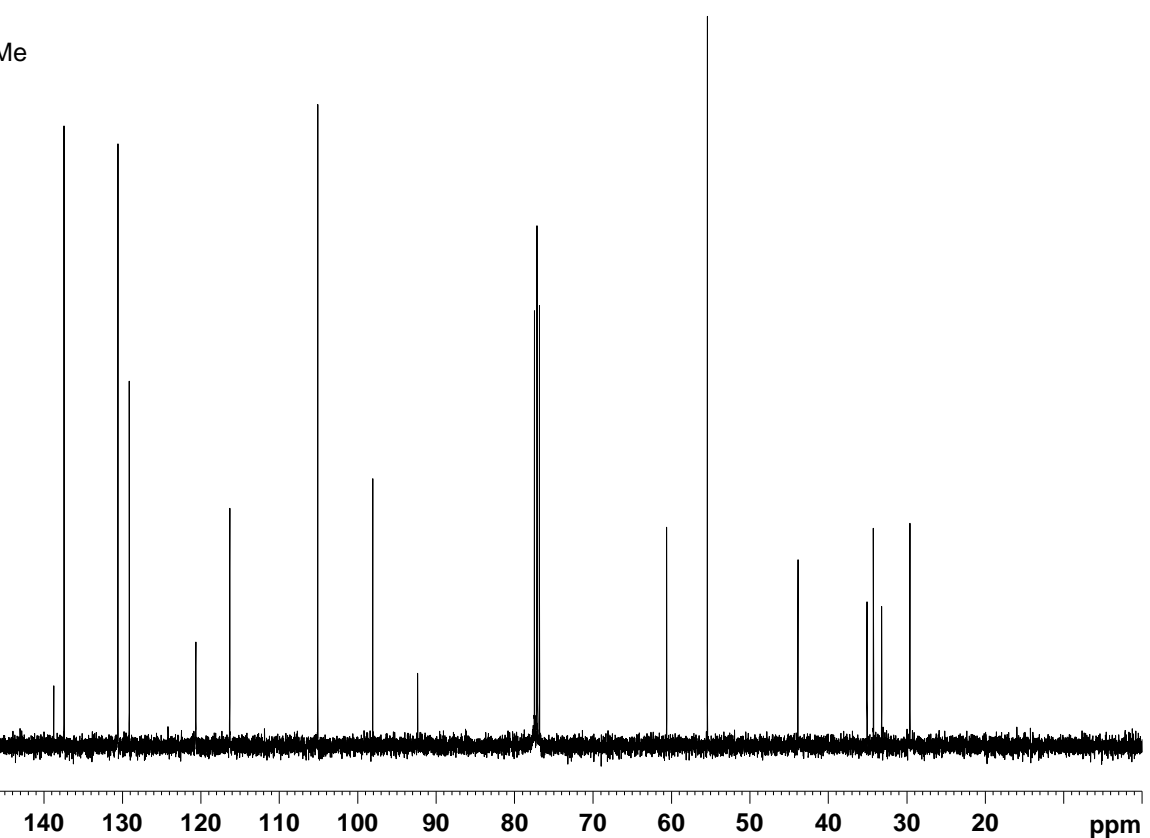


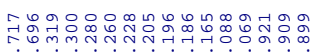

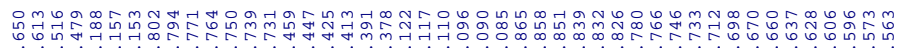

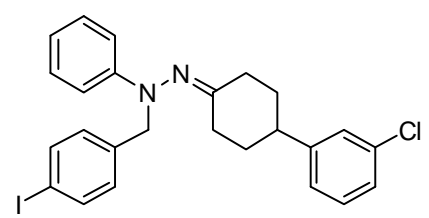

1n
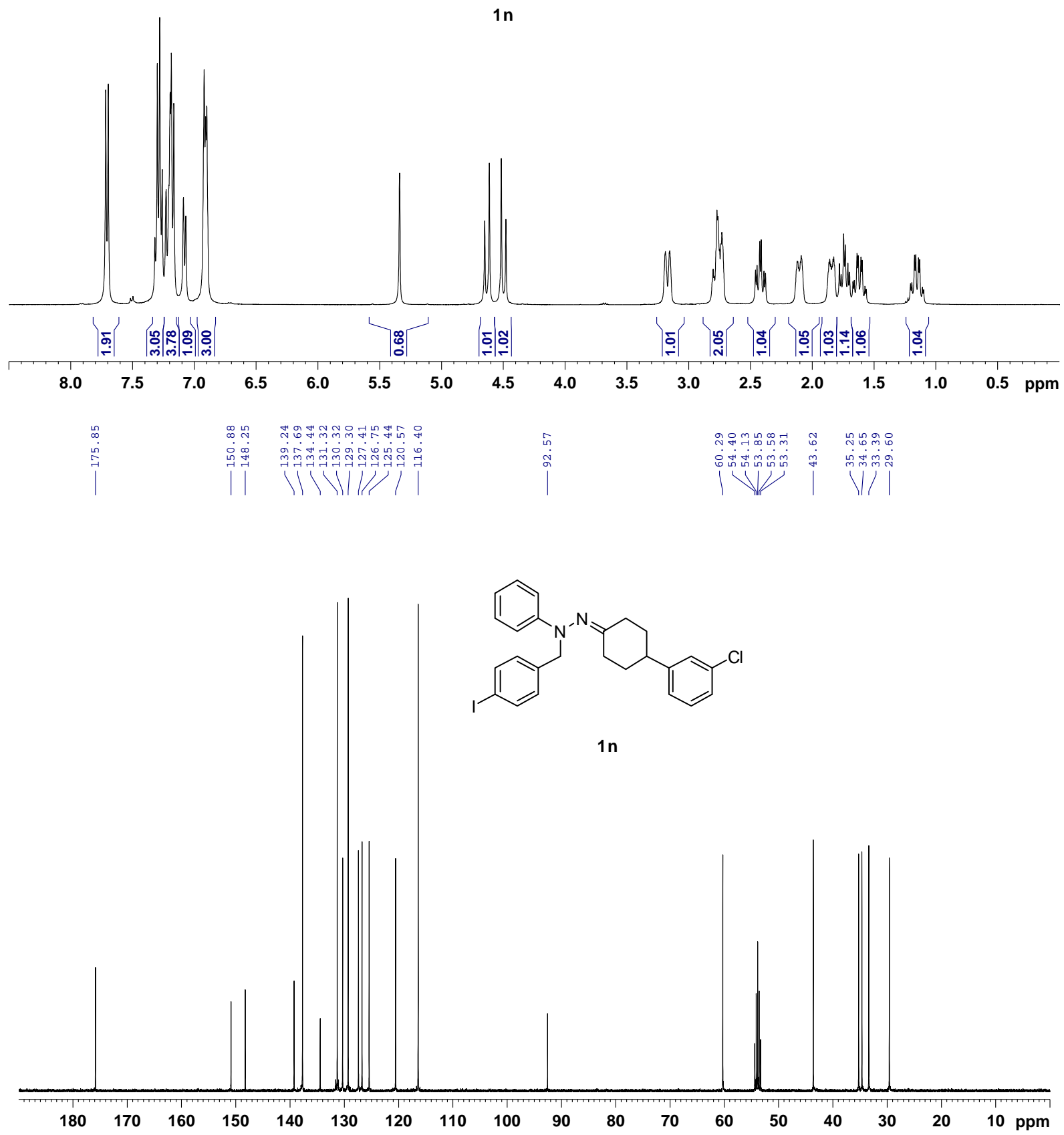

S77 

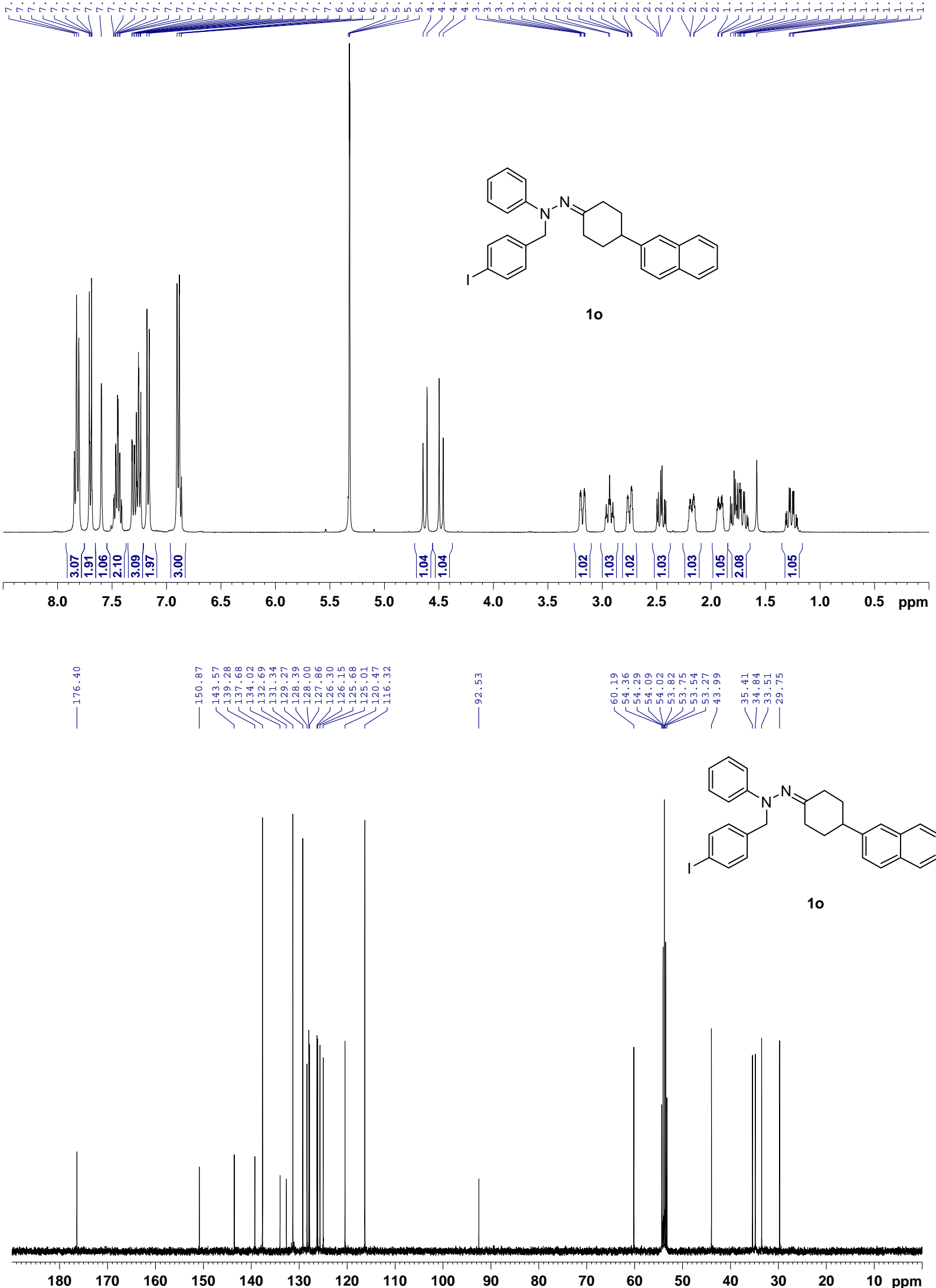


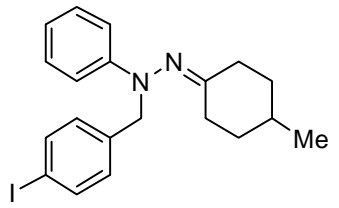

$1 \mathrm{p}$
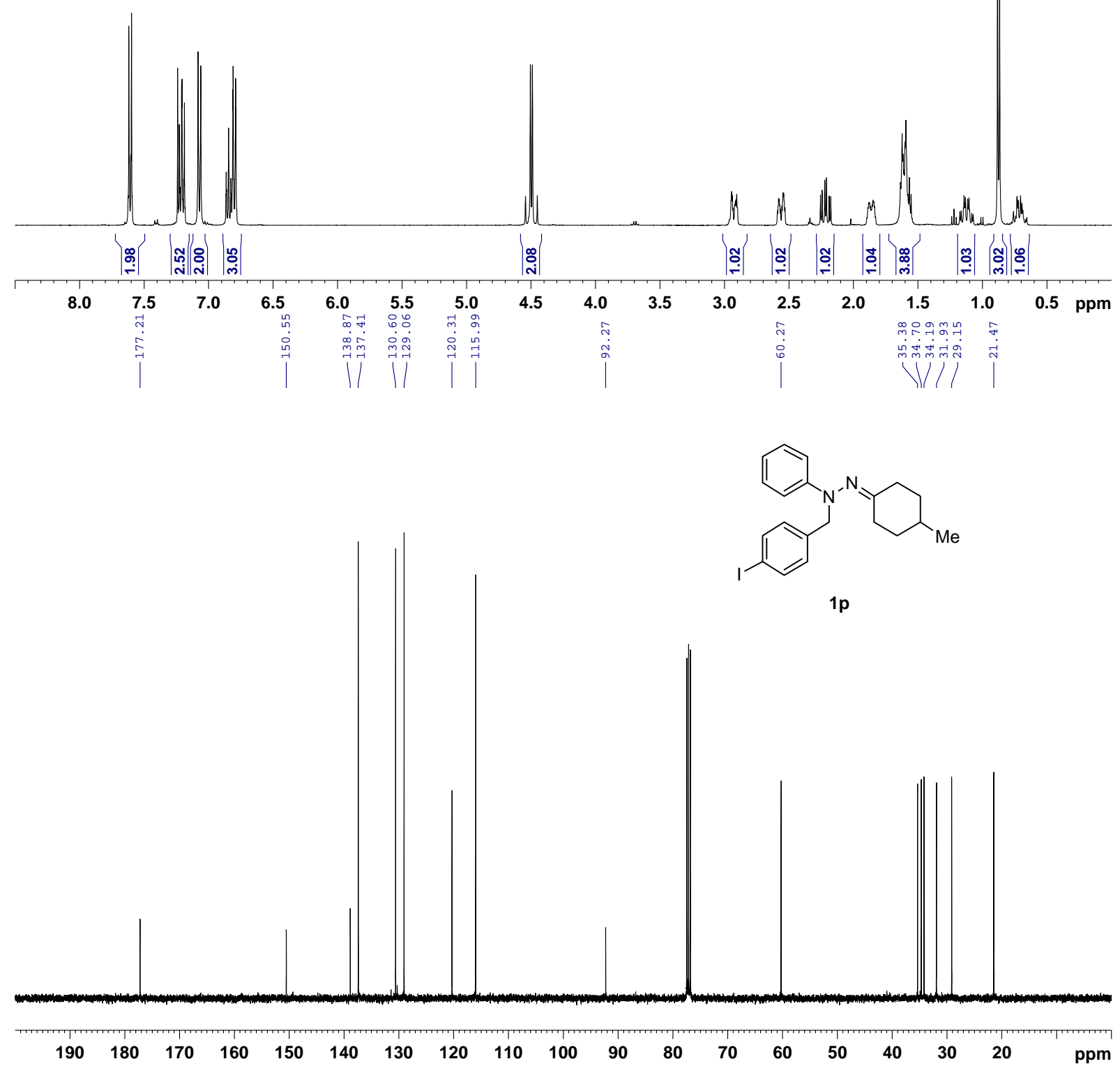

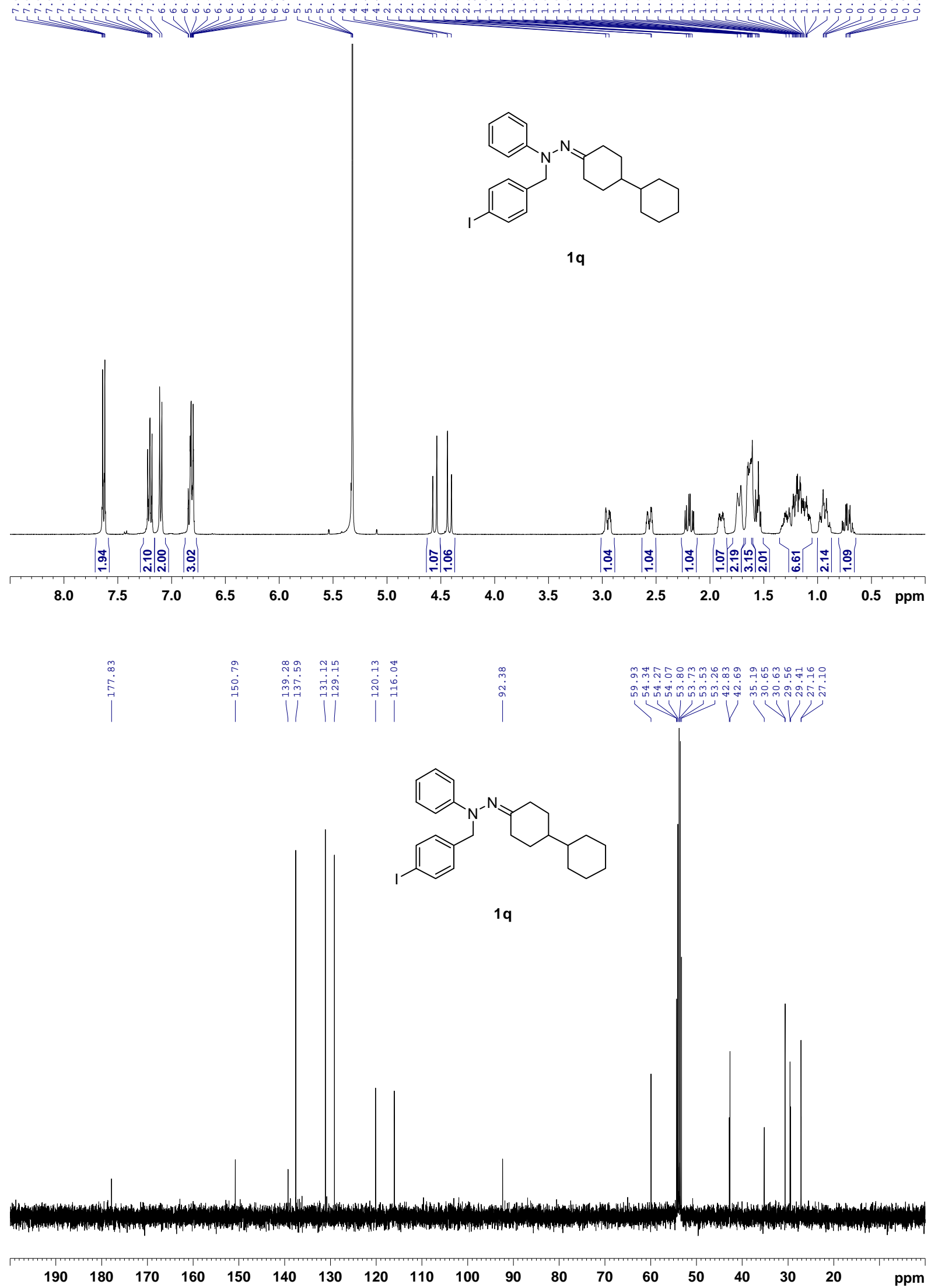


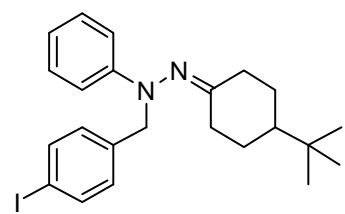

$1 \mathrm{r}$
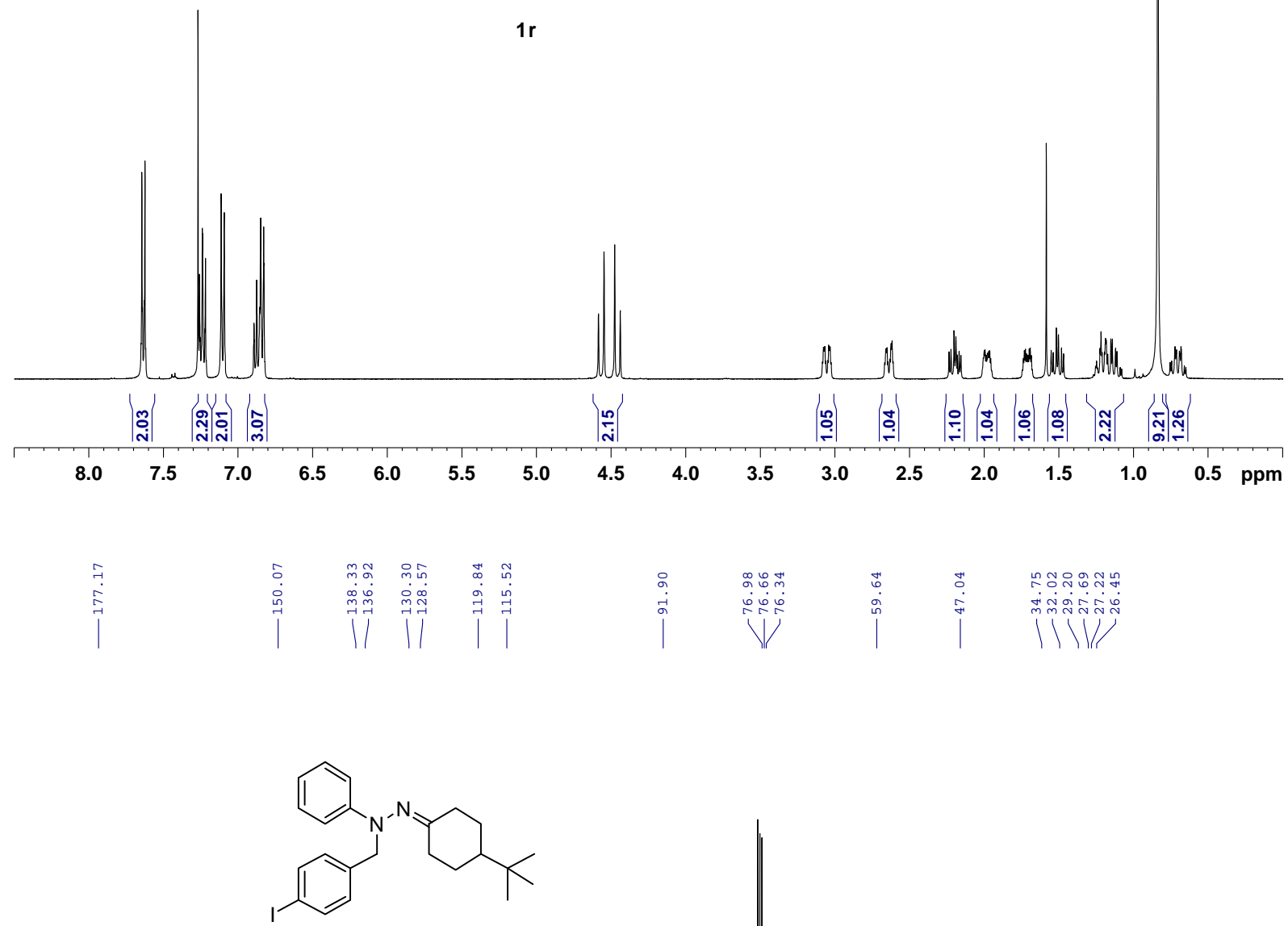

$1 \mathrm{r}$

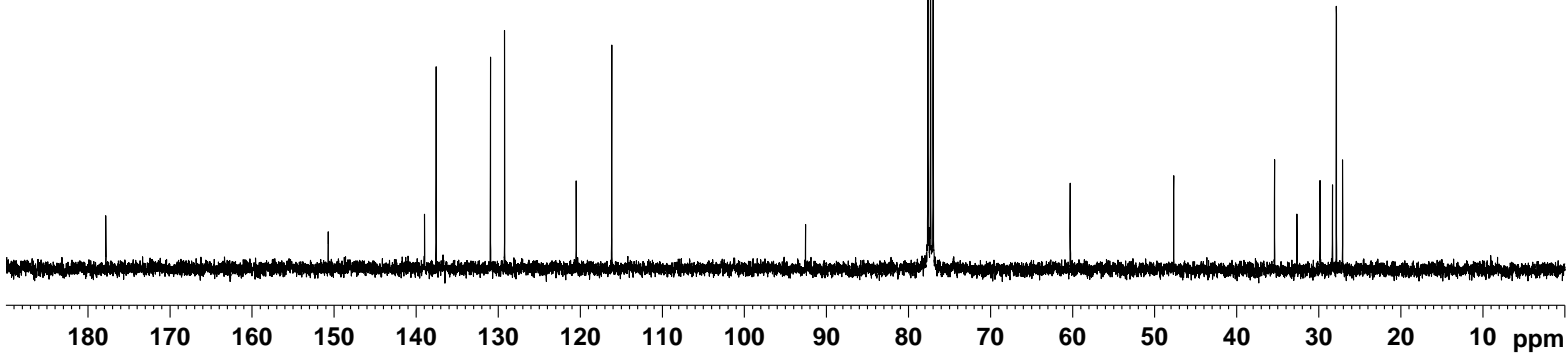




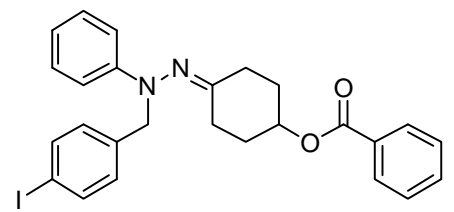

1s
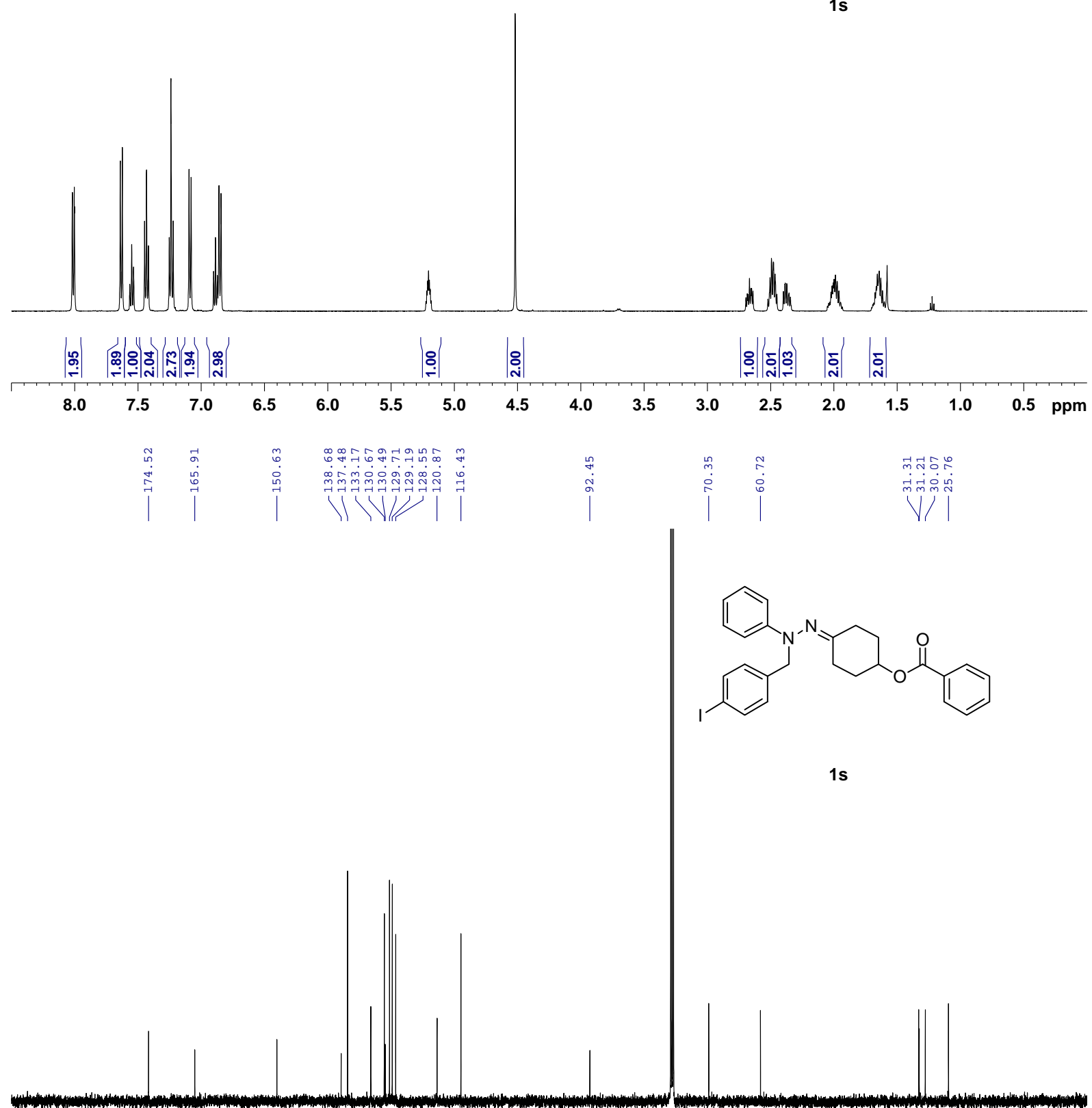

15

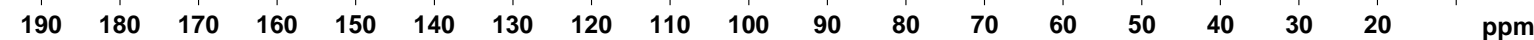




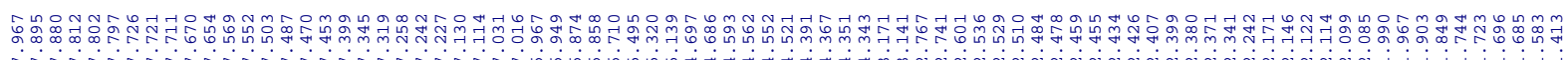
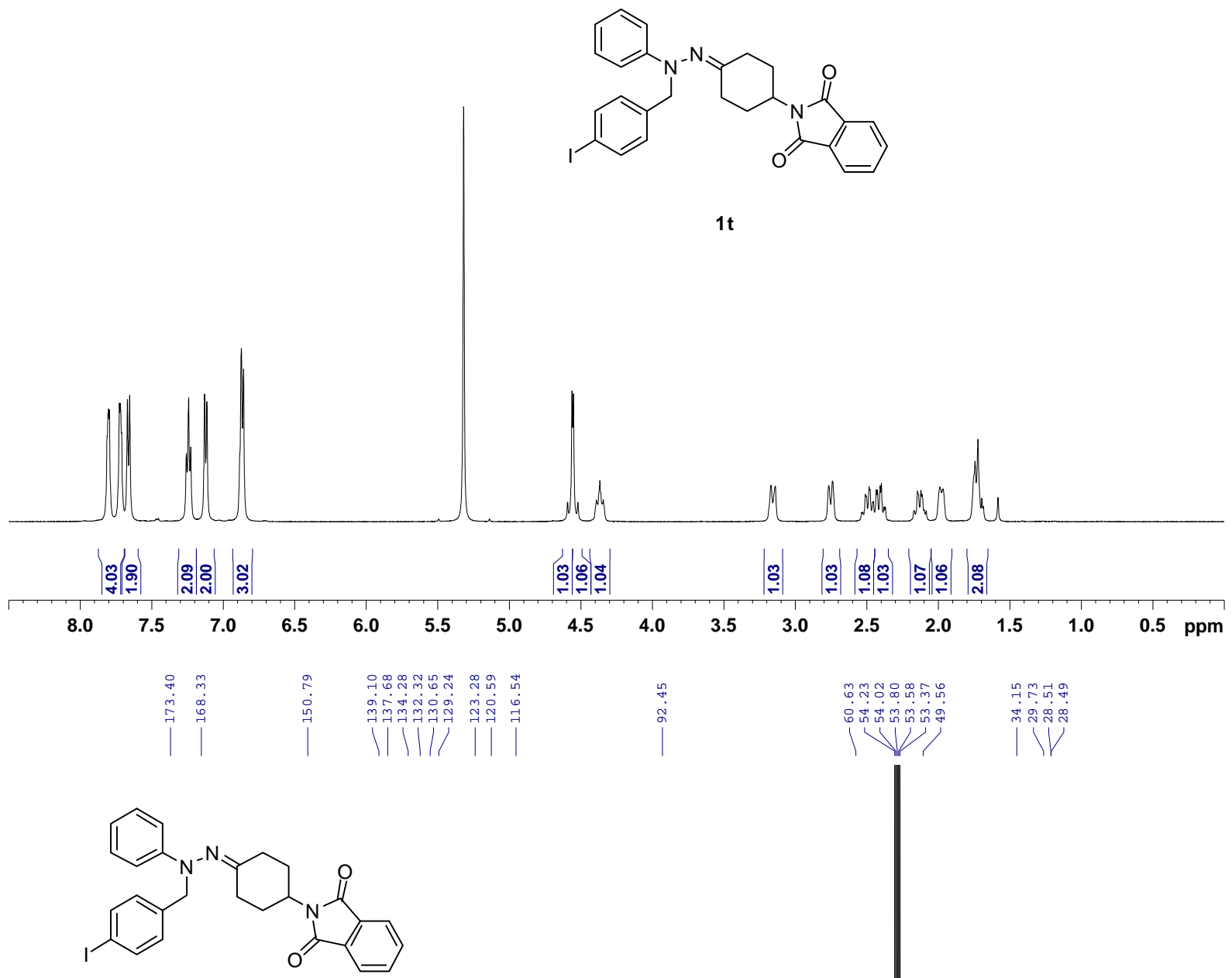

$1 \mathbf{t}$

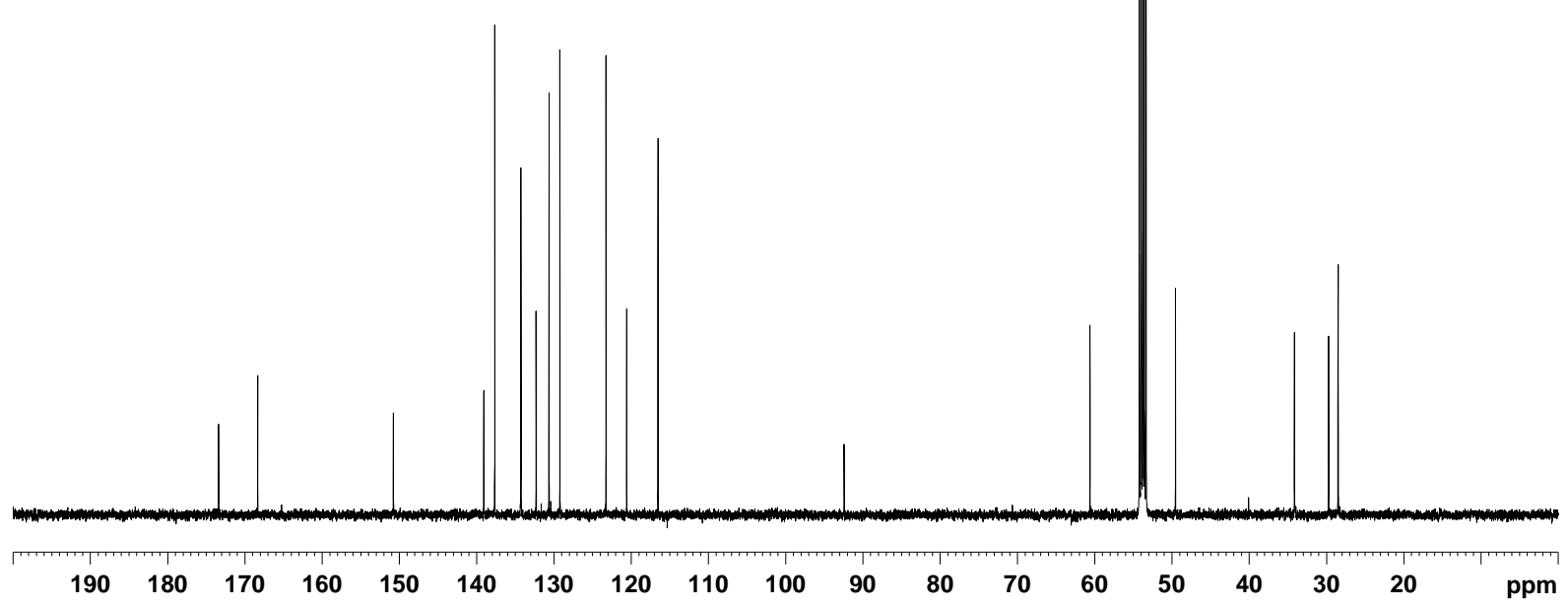

S83 


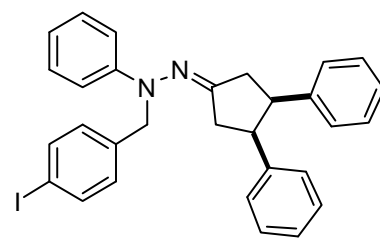

1v
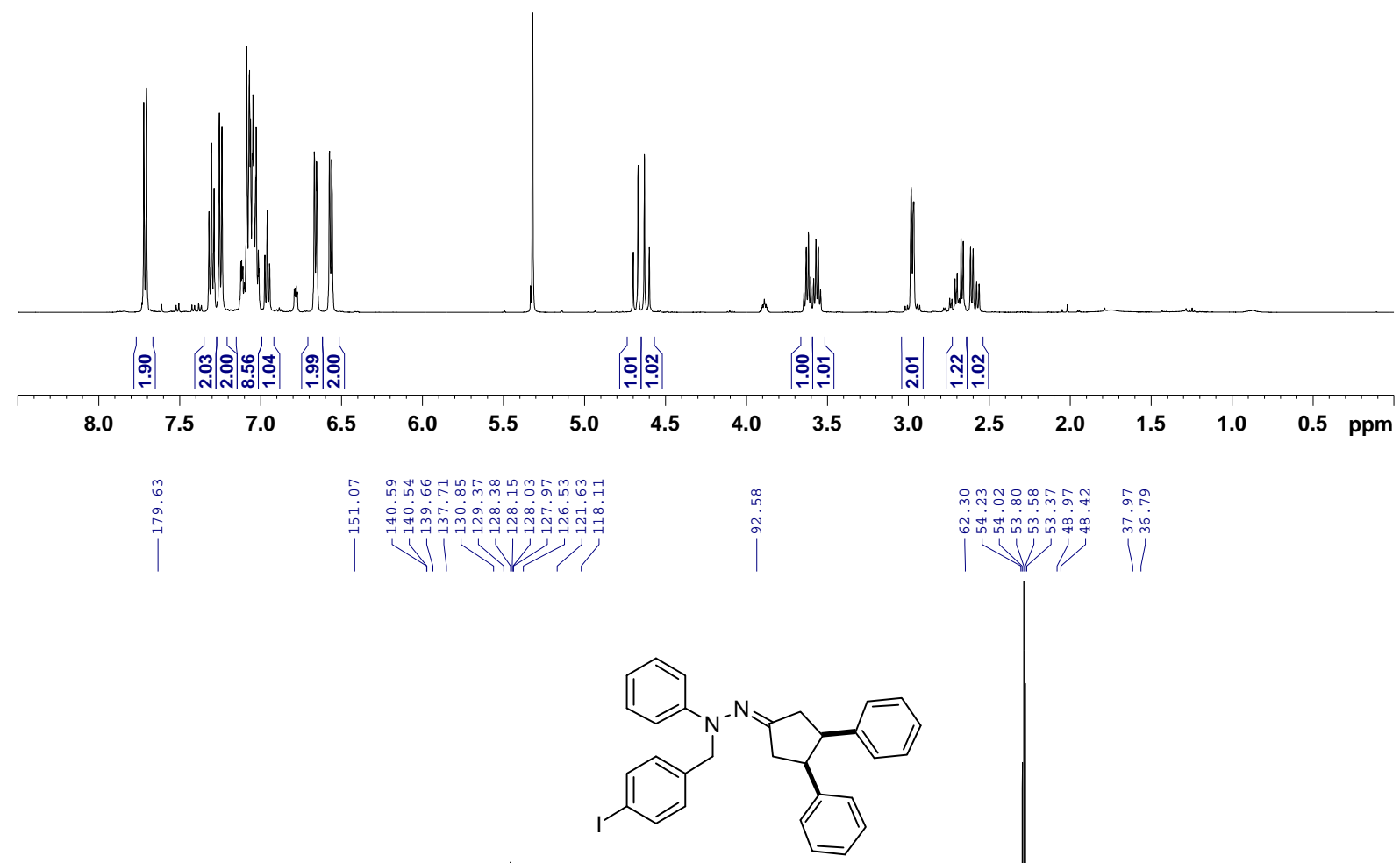

1v

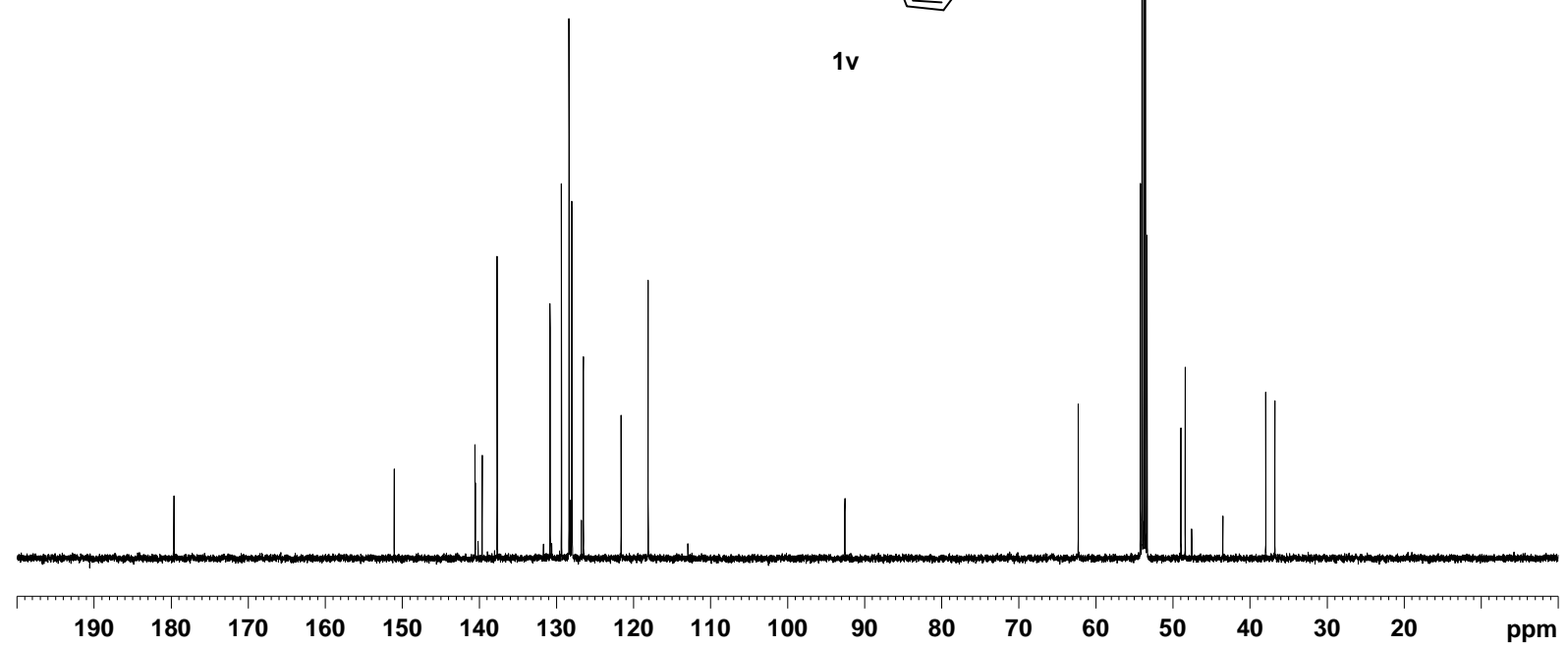



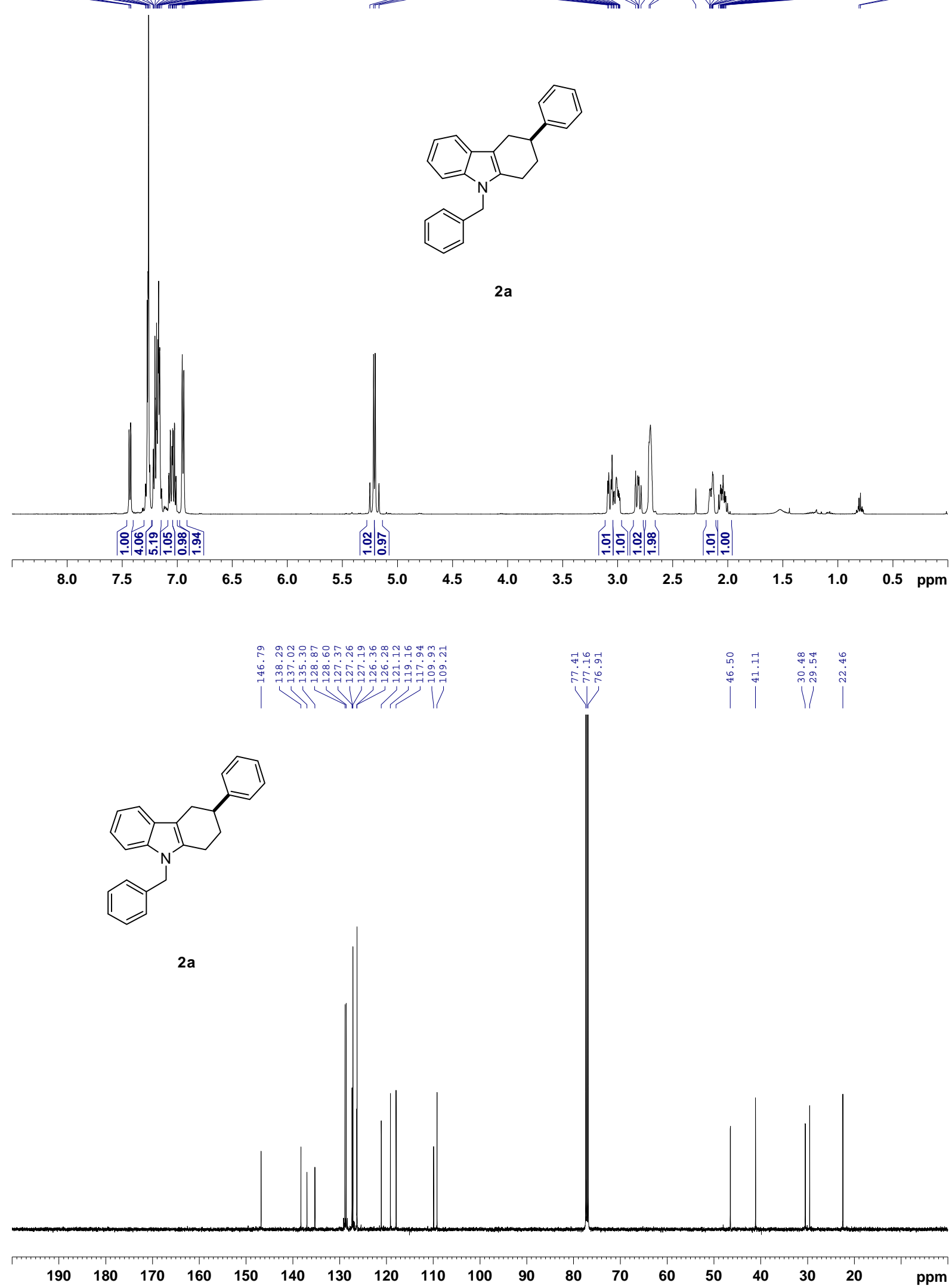


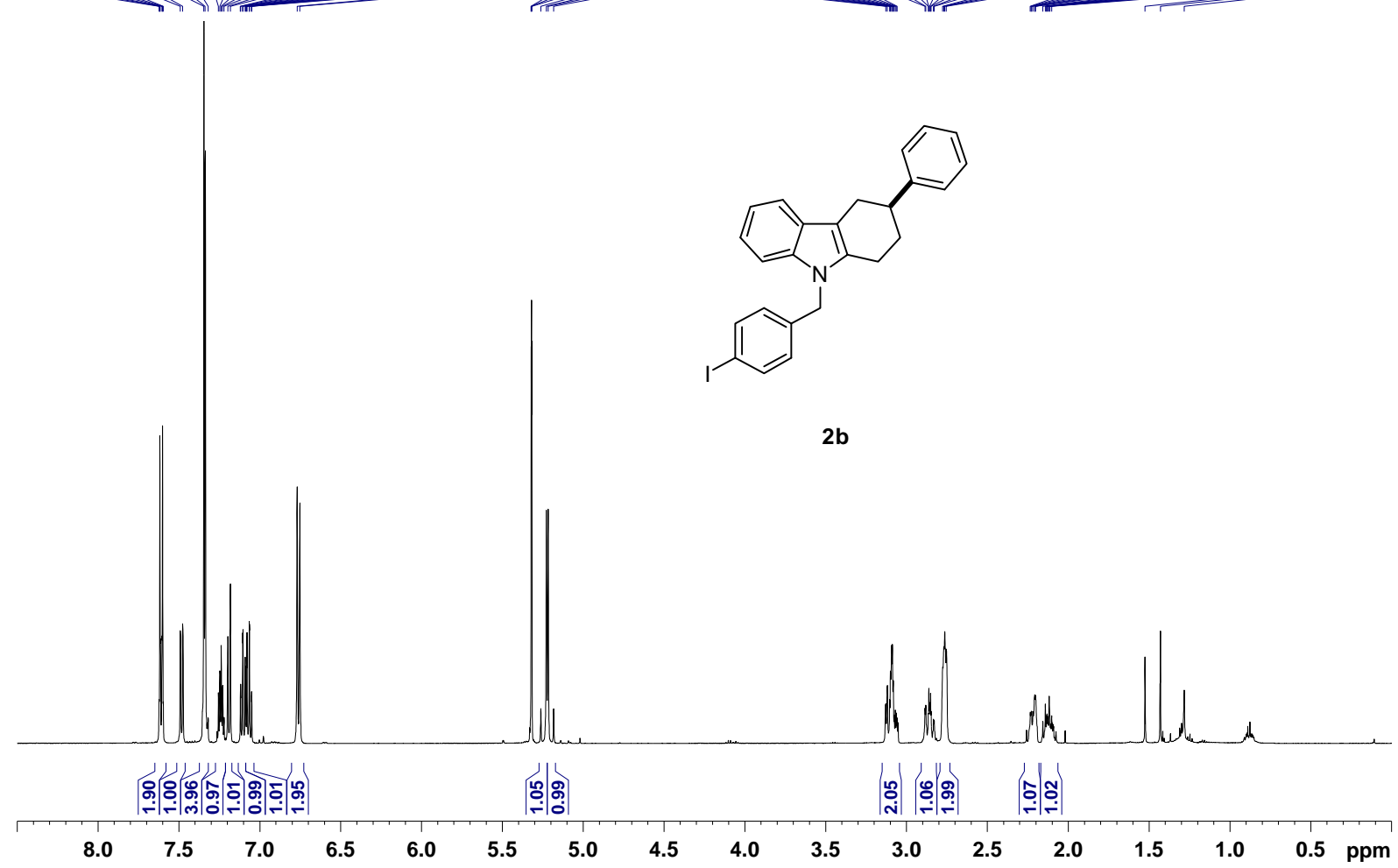

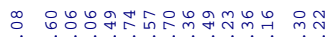

守

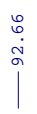

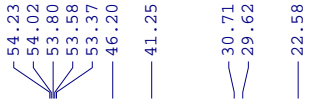

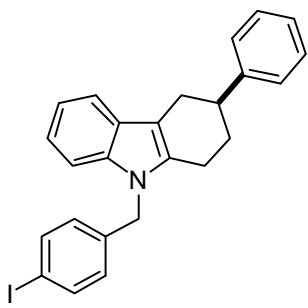

$2 \mathbf{b}$

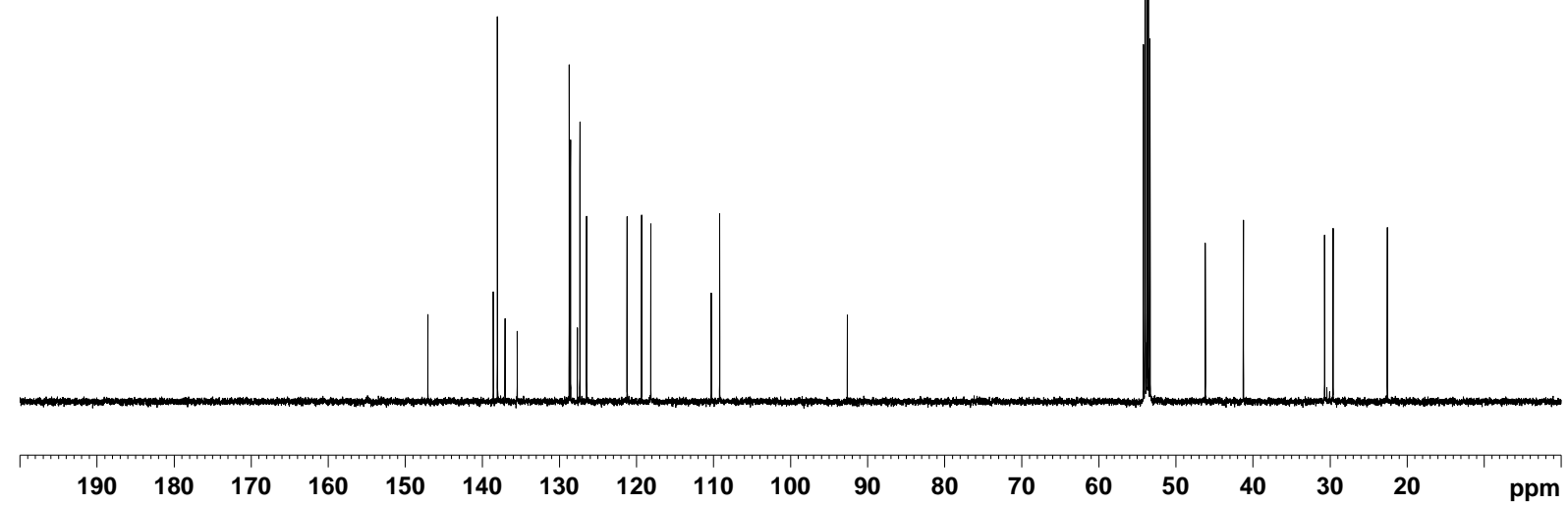




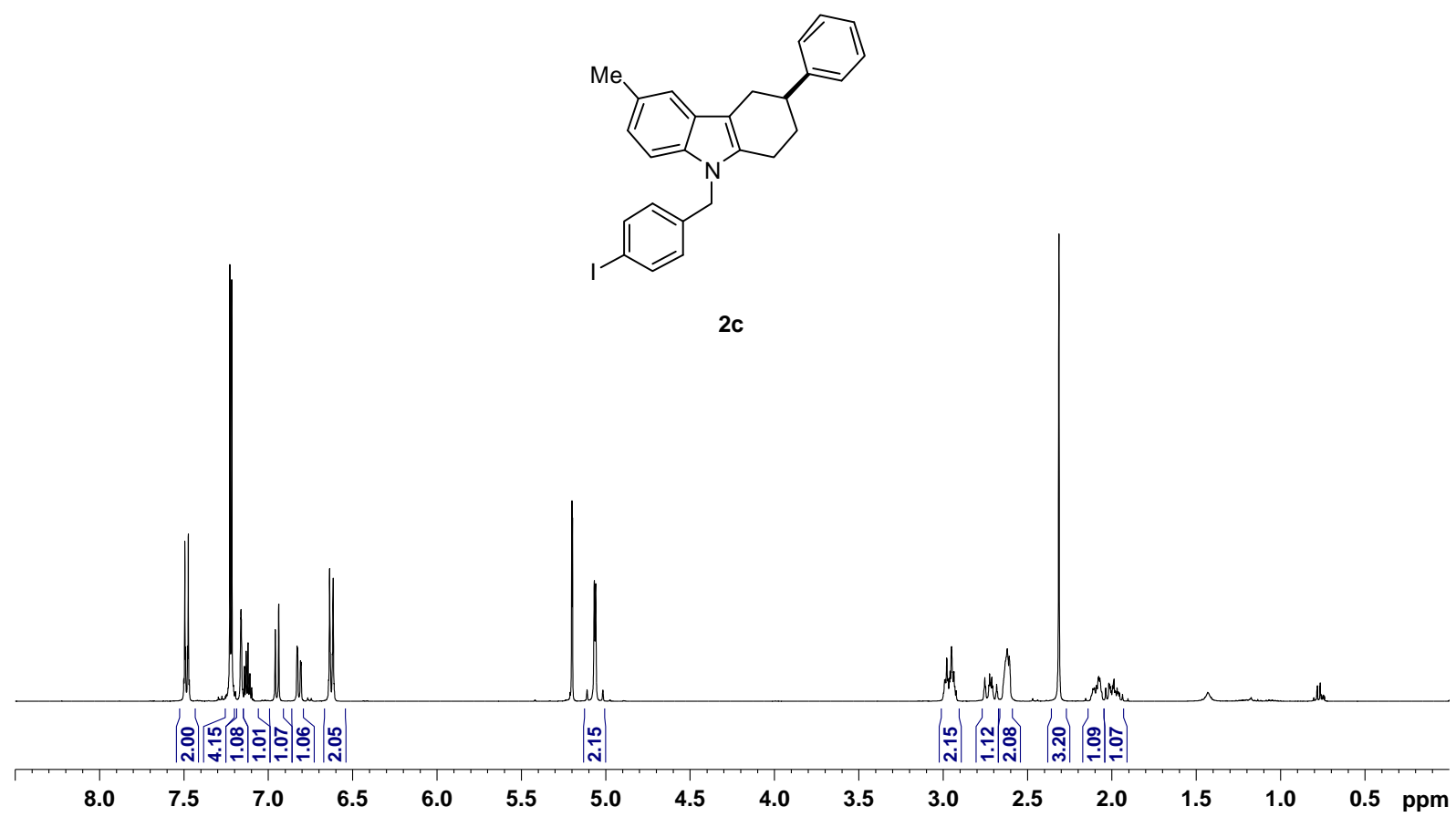

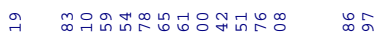

|

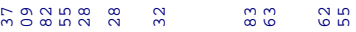

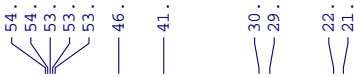

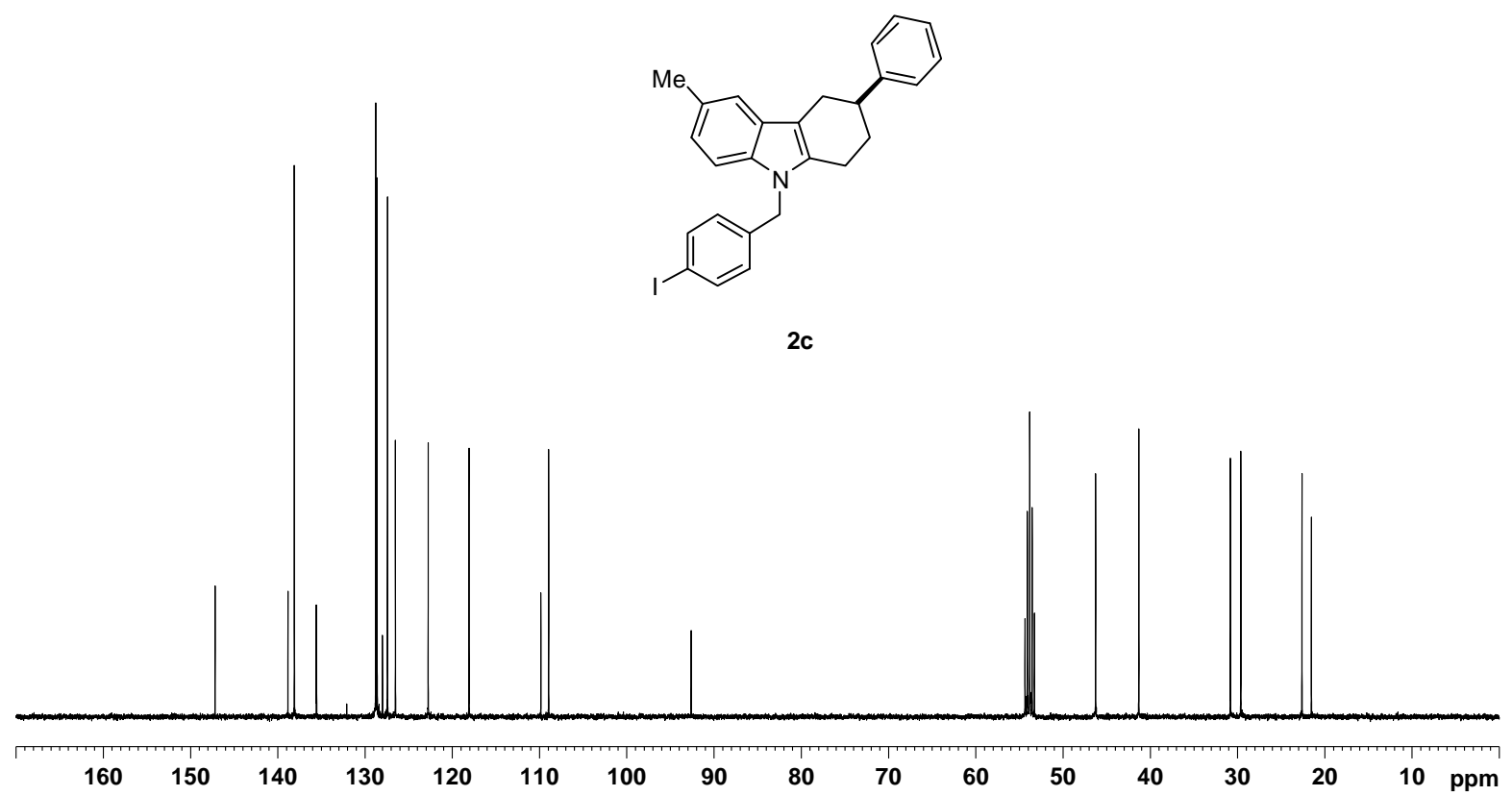




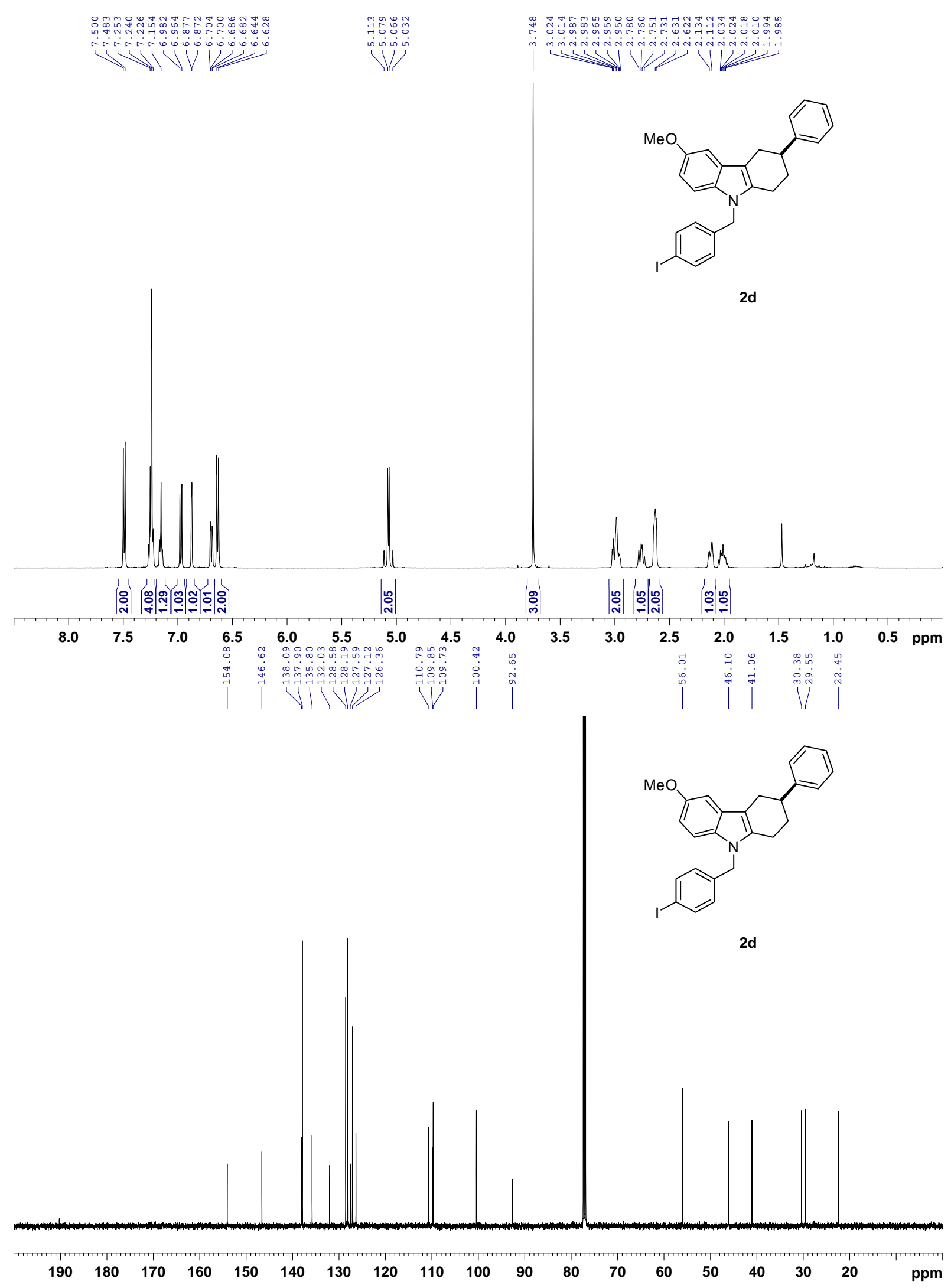




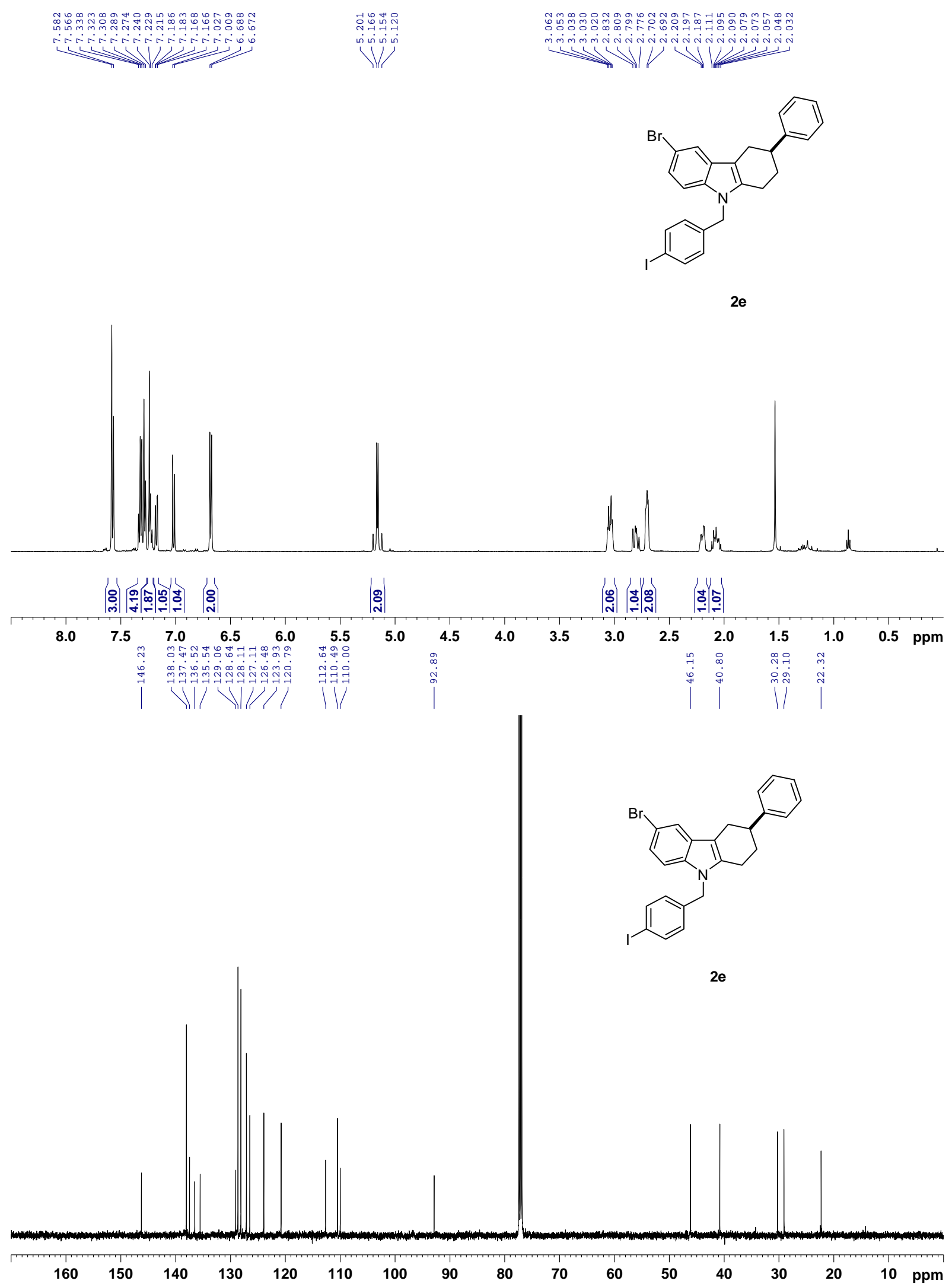




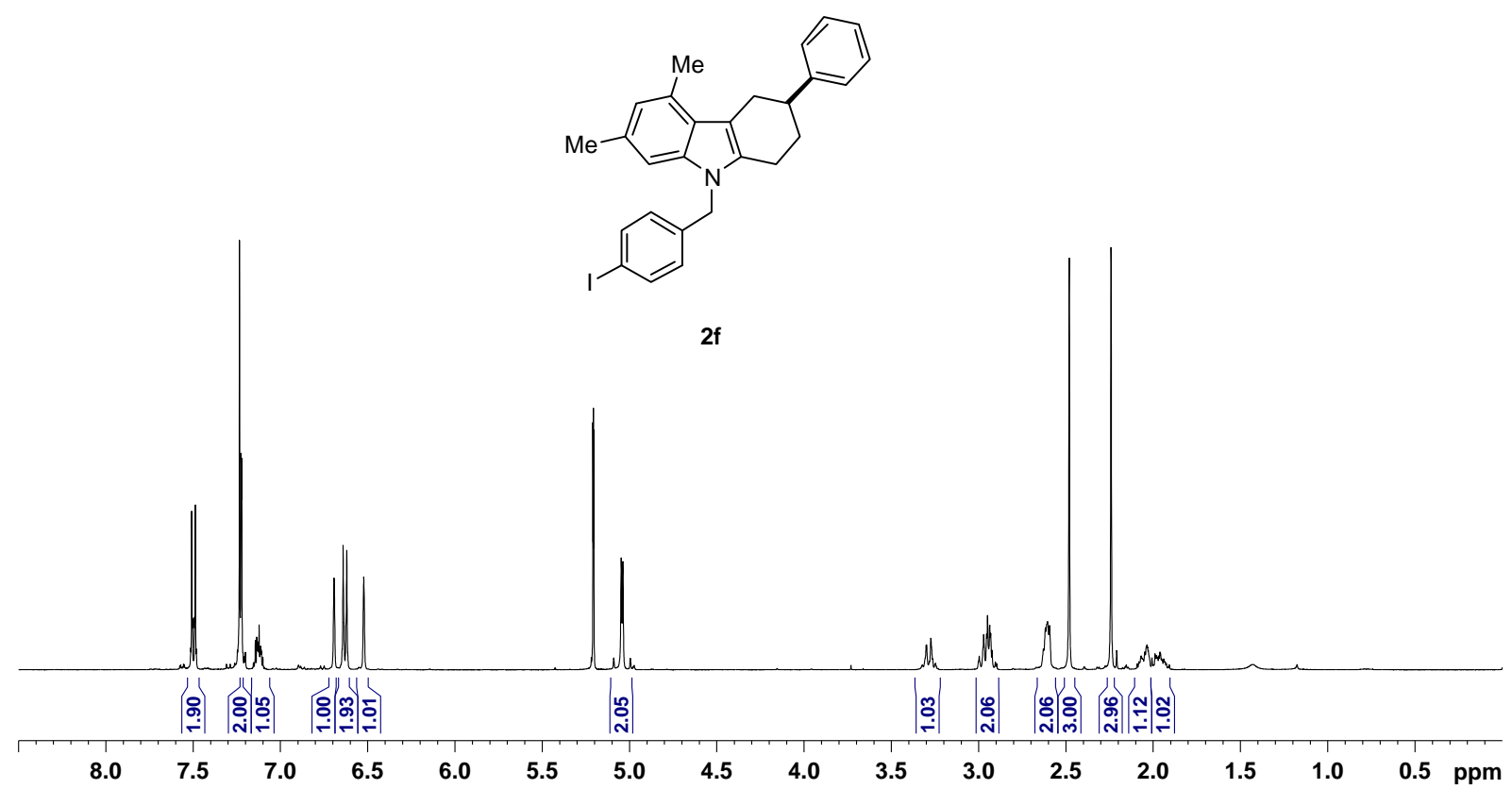

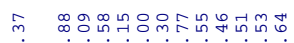

守

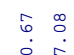

국

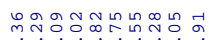

(N)
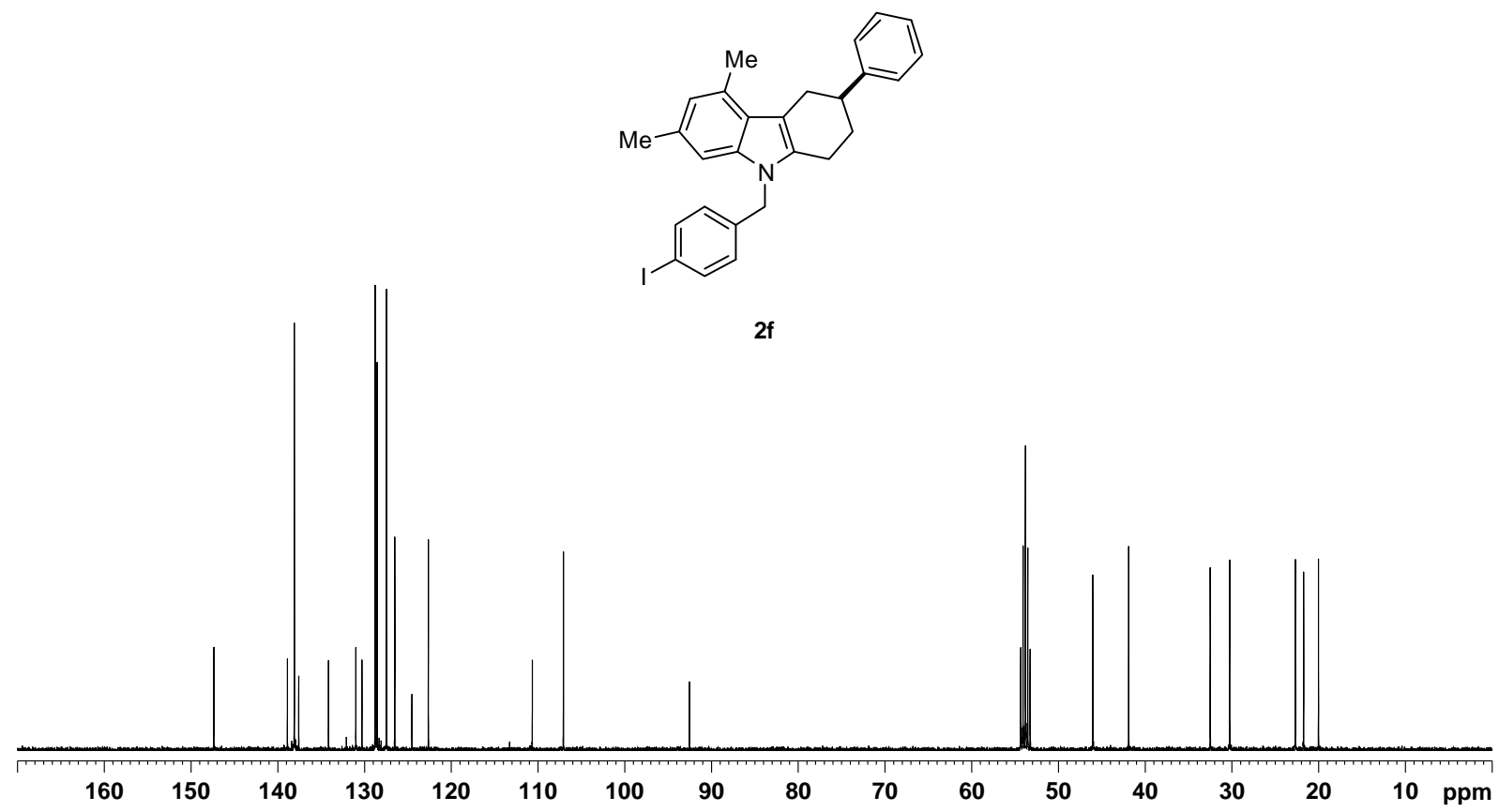

$2 f$ 


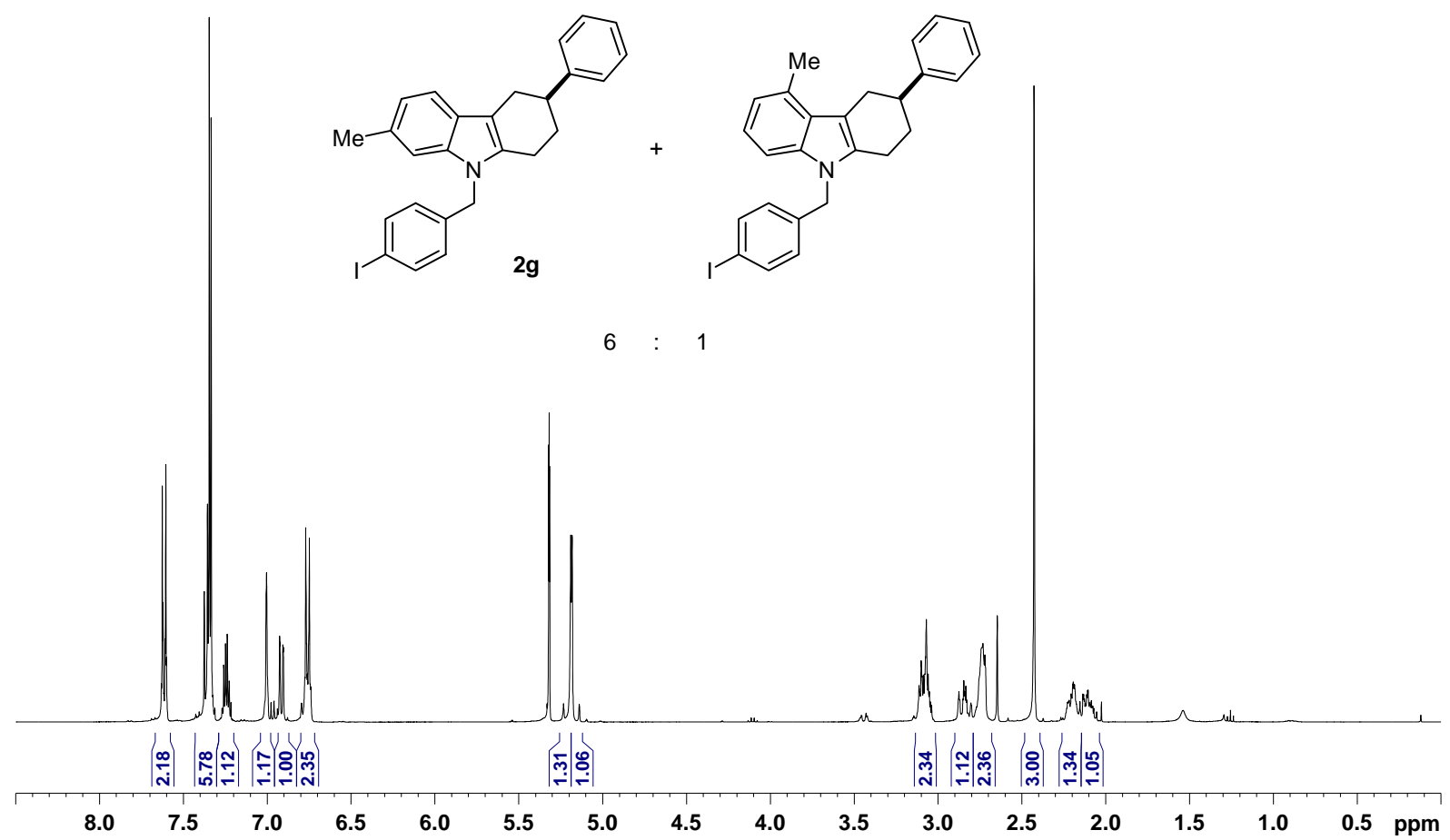

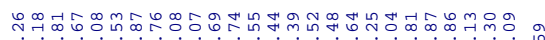

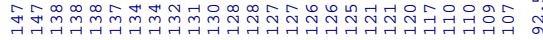
$\underbrace{-1}$

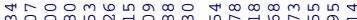

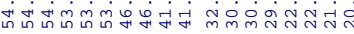
WV VIV YI/Y

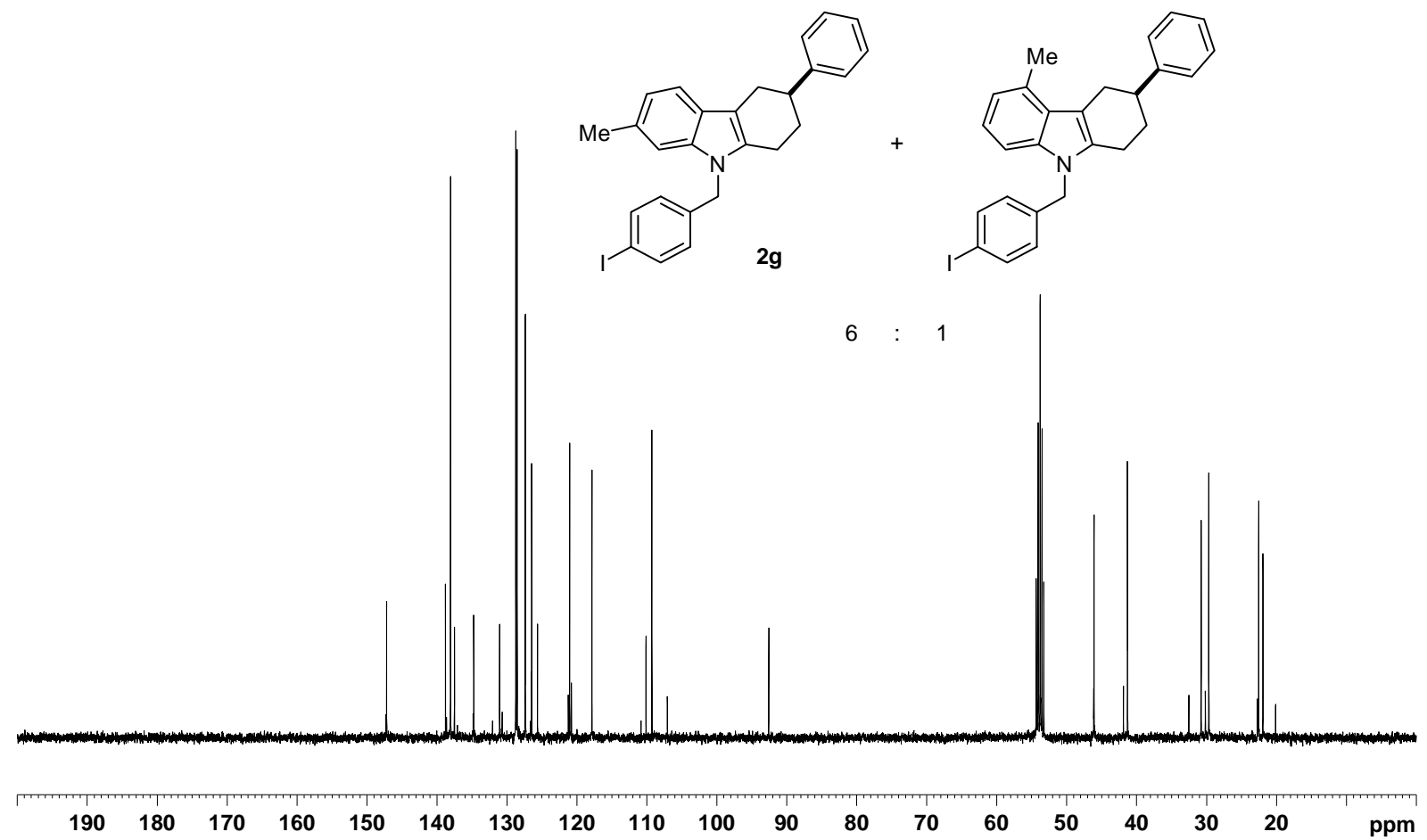



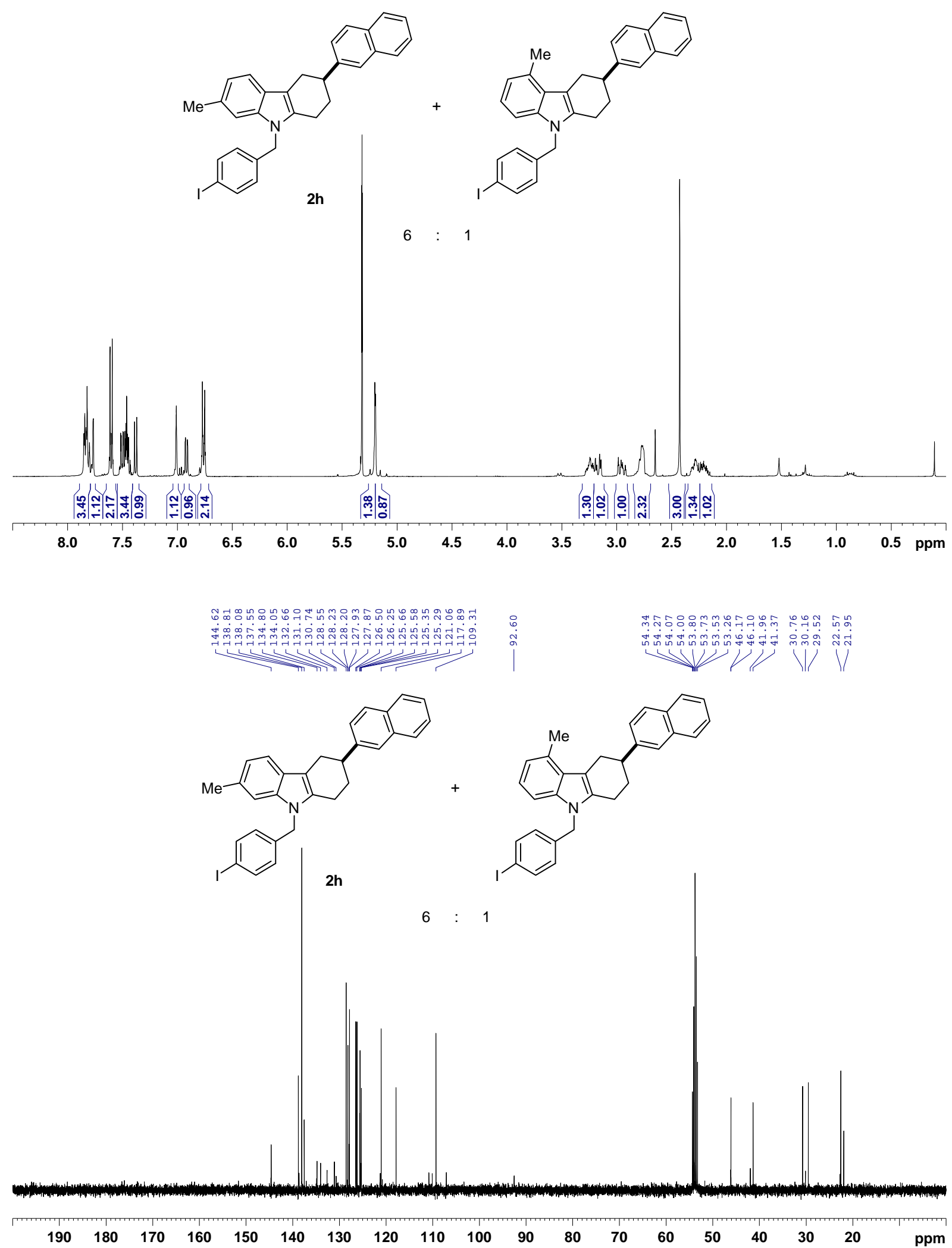


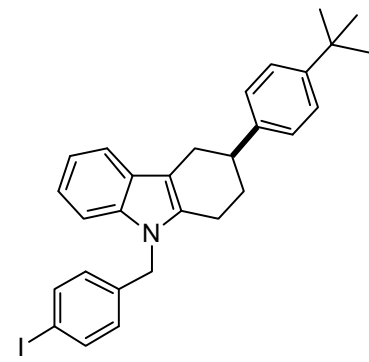

2i

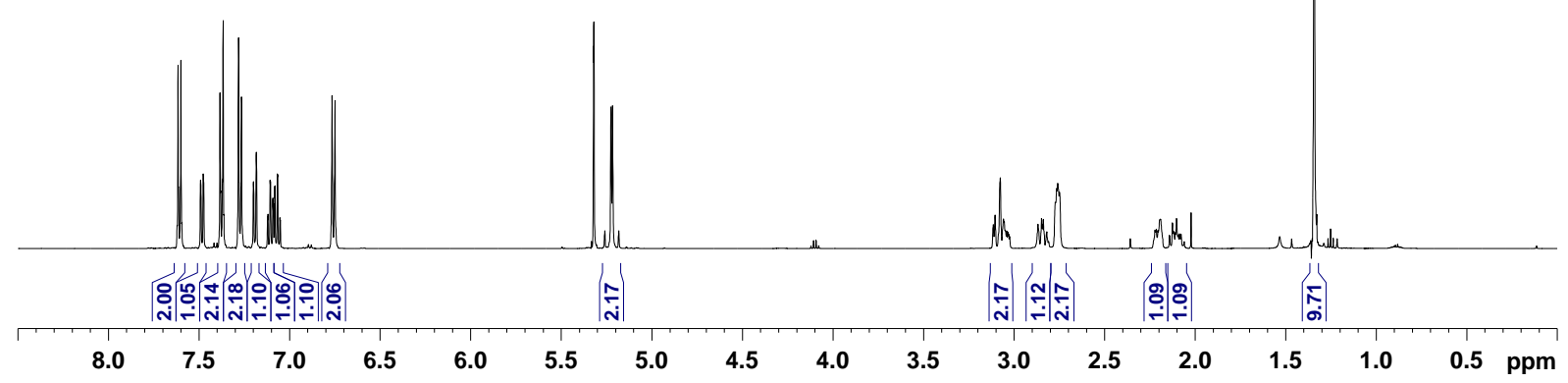

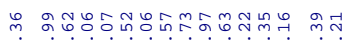
离

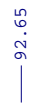
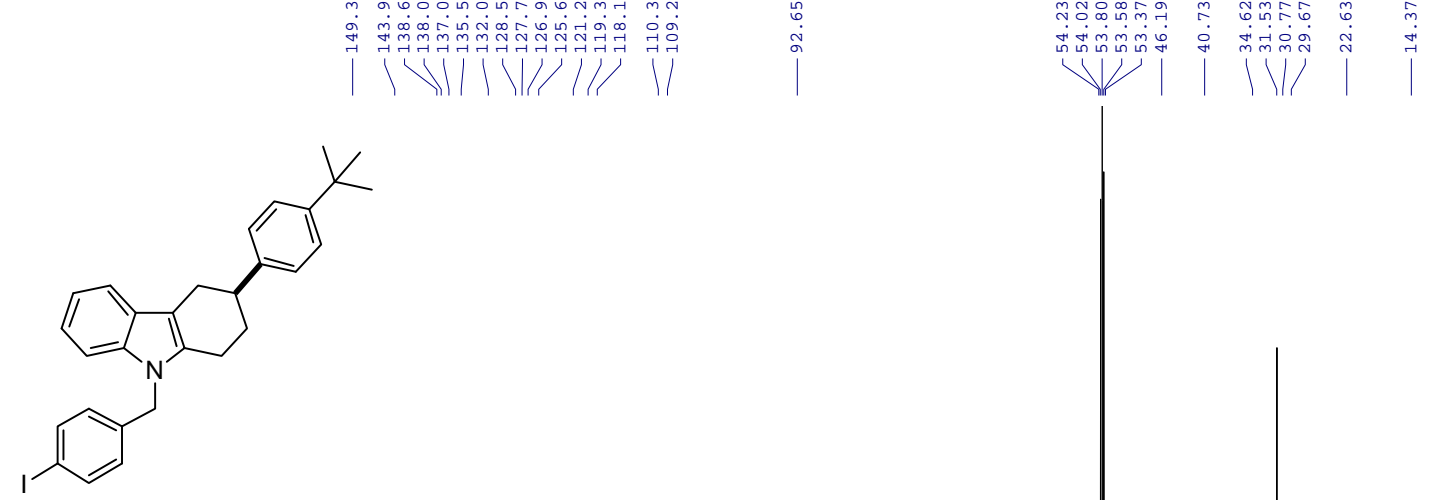

2i

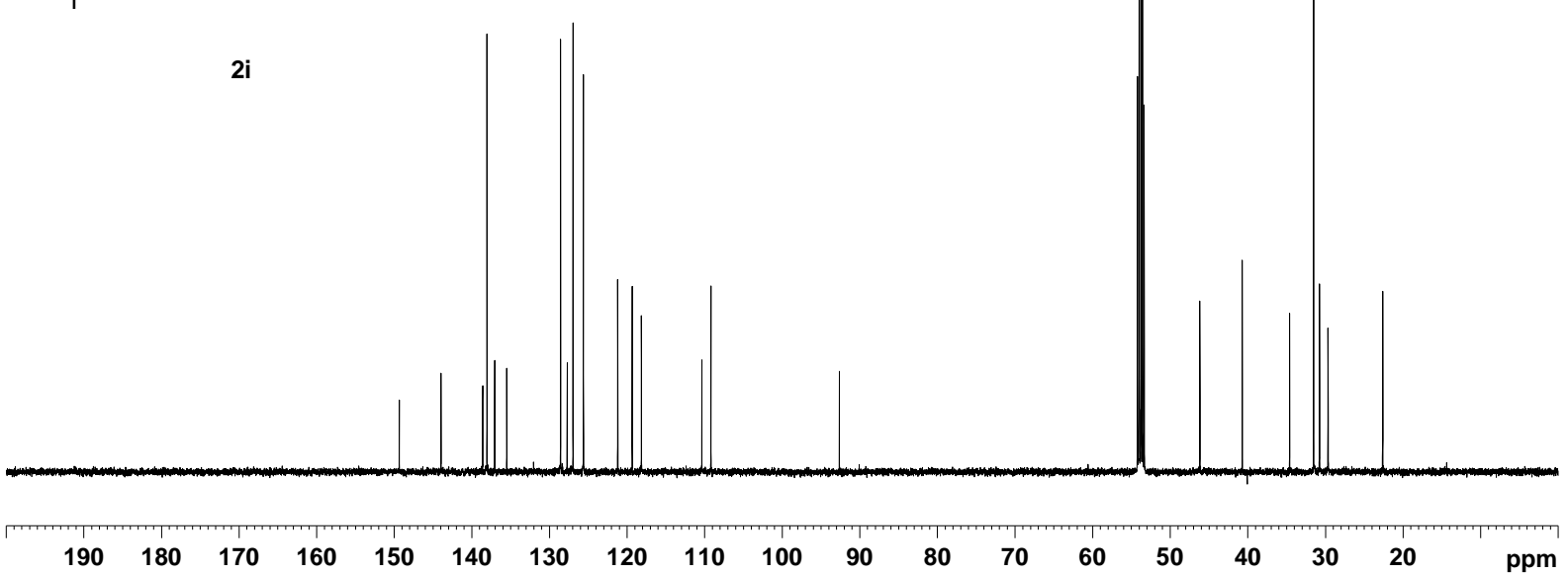




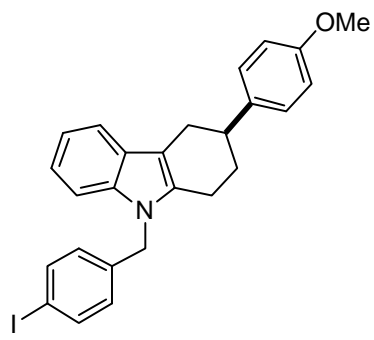

2j

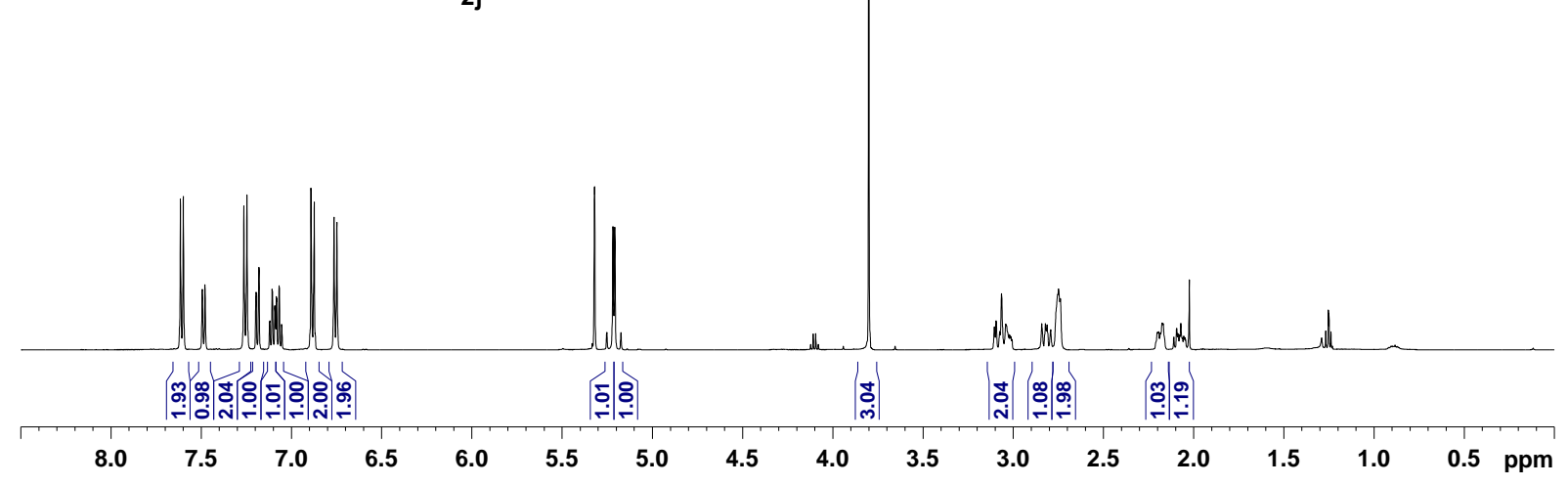

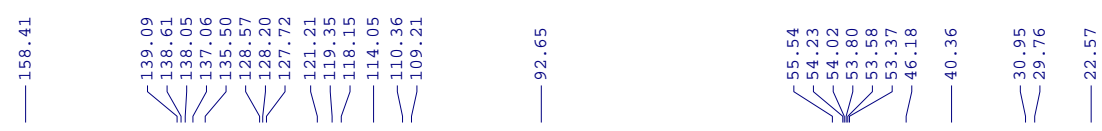

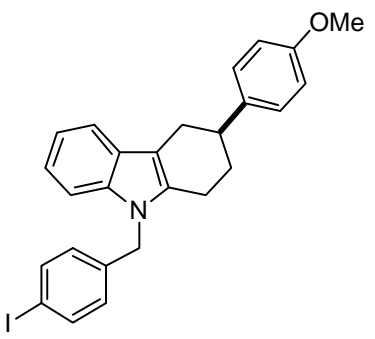

$2 \mathrm{j}$

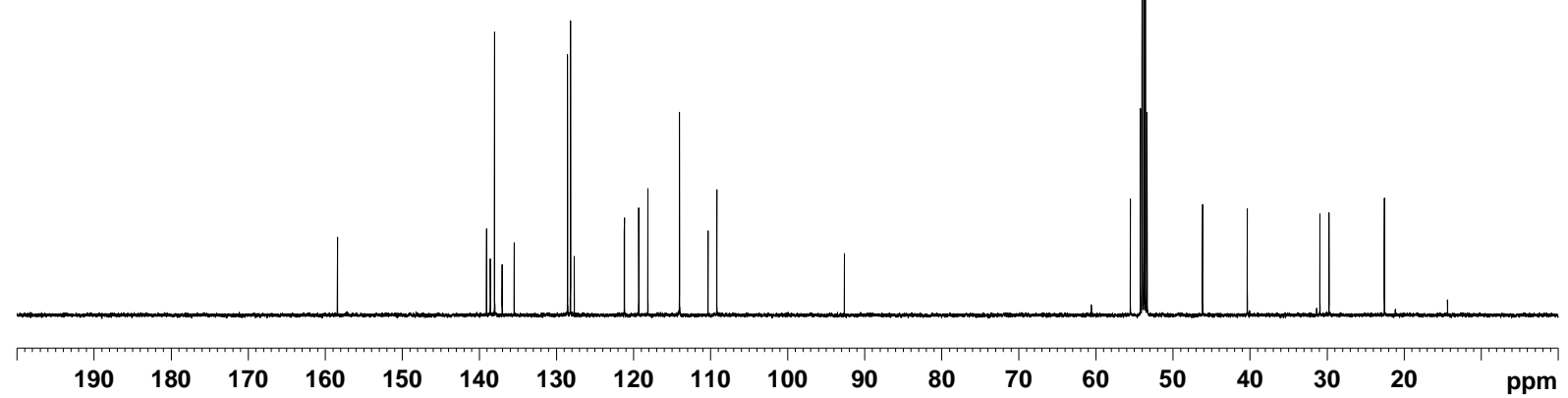




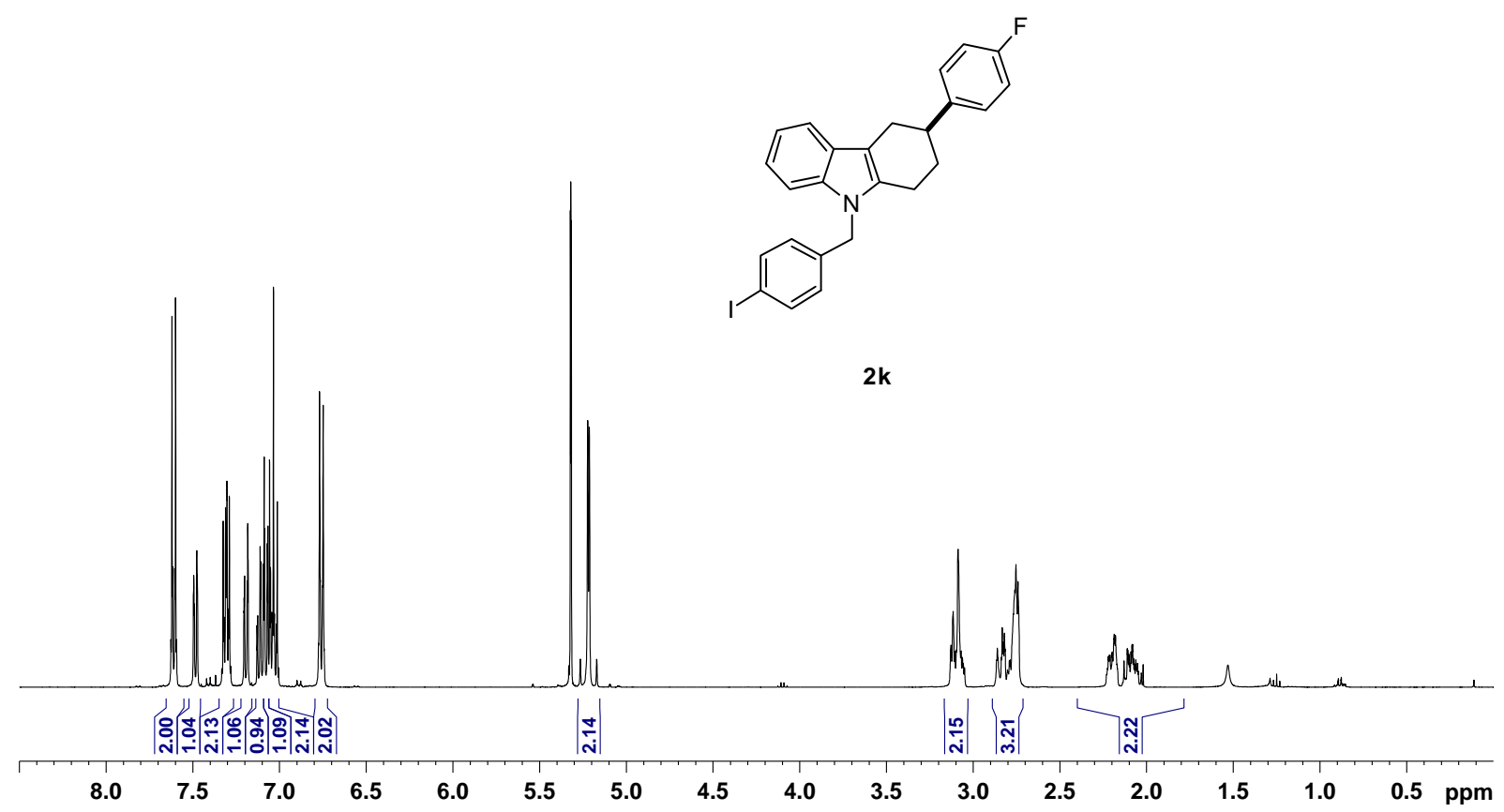

\begin{tabular}{|c|c|c|c|c|}
\hline ลิ่ & চ & 4 & 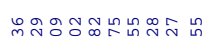 & $\infty_{\infty}^{n}$ \\
\hline 용 & 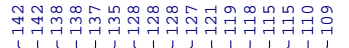 & $\dot{\alpha}$ & 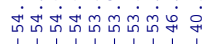 & ஜ் \\
\hline
\end{tabular}

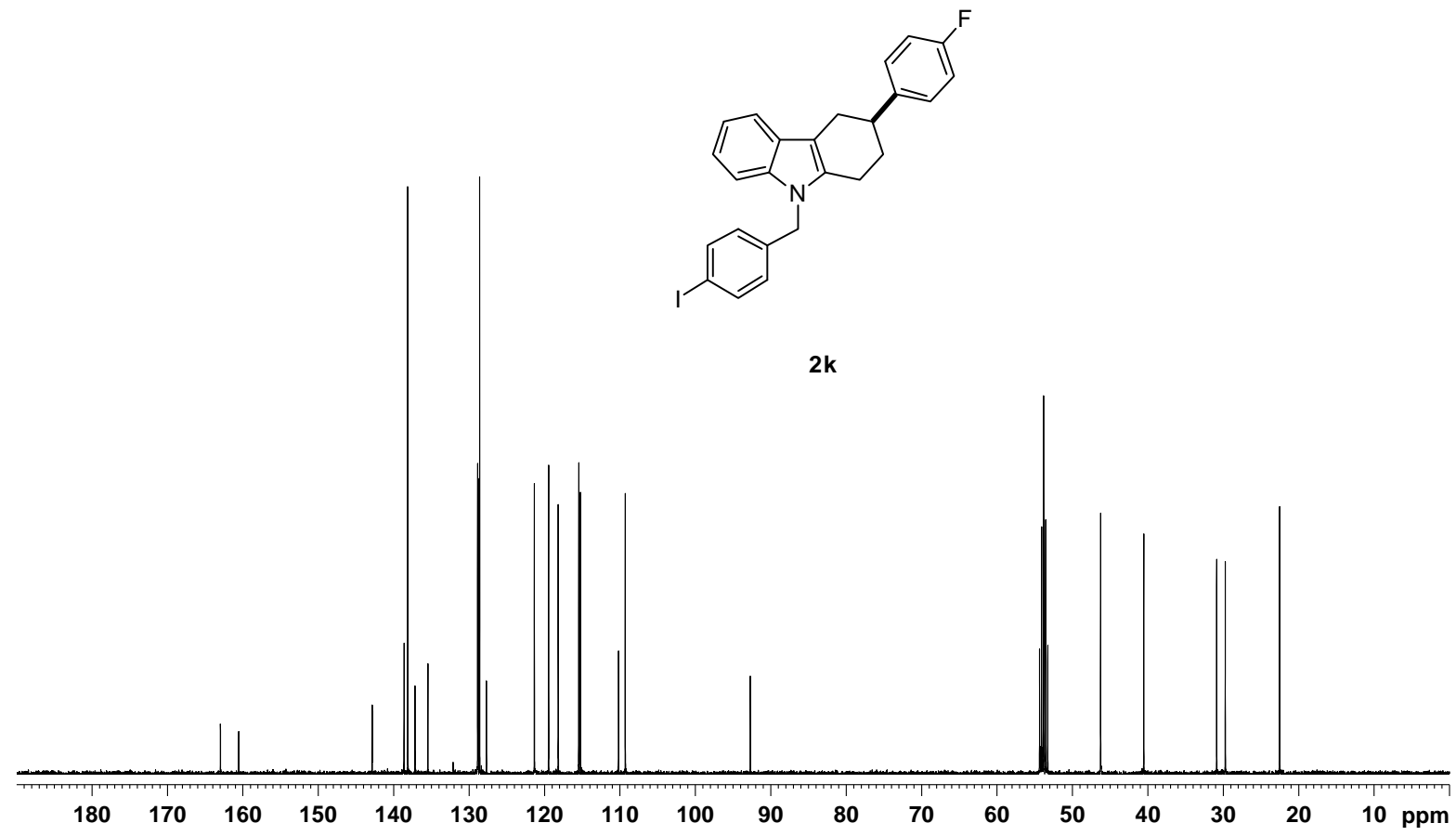




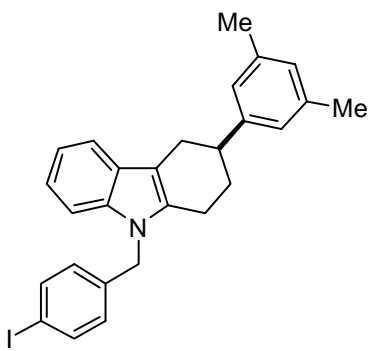

2I

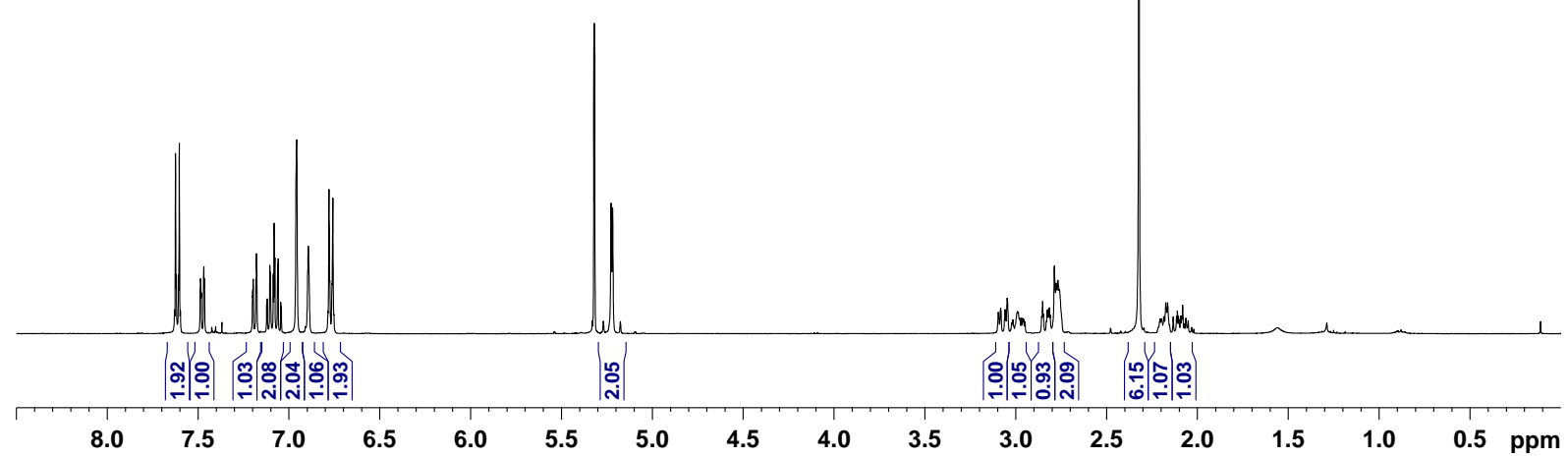

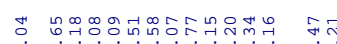
要

$\dddot{\wp}$

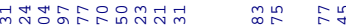

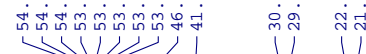

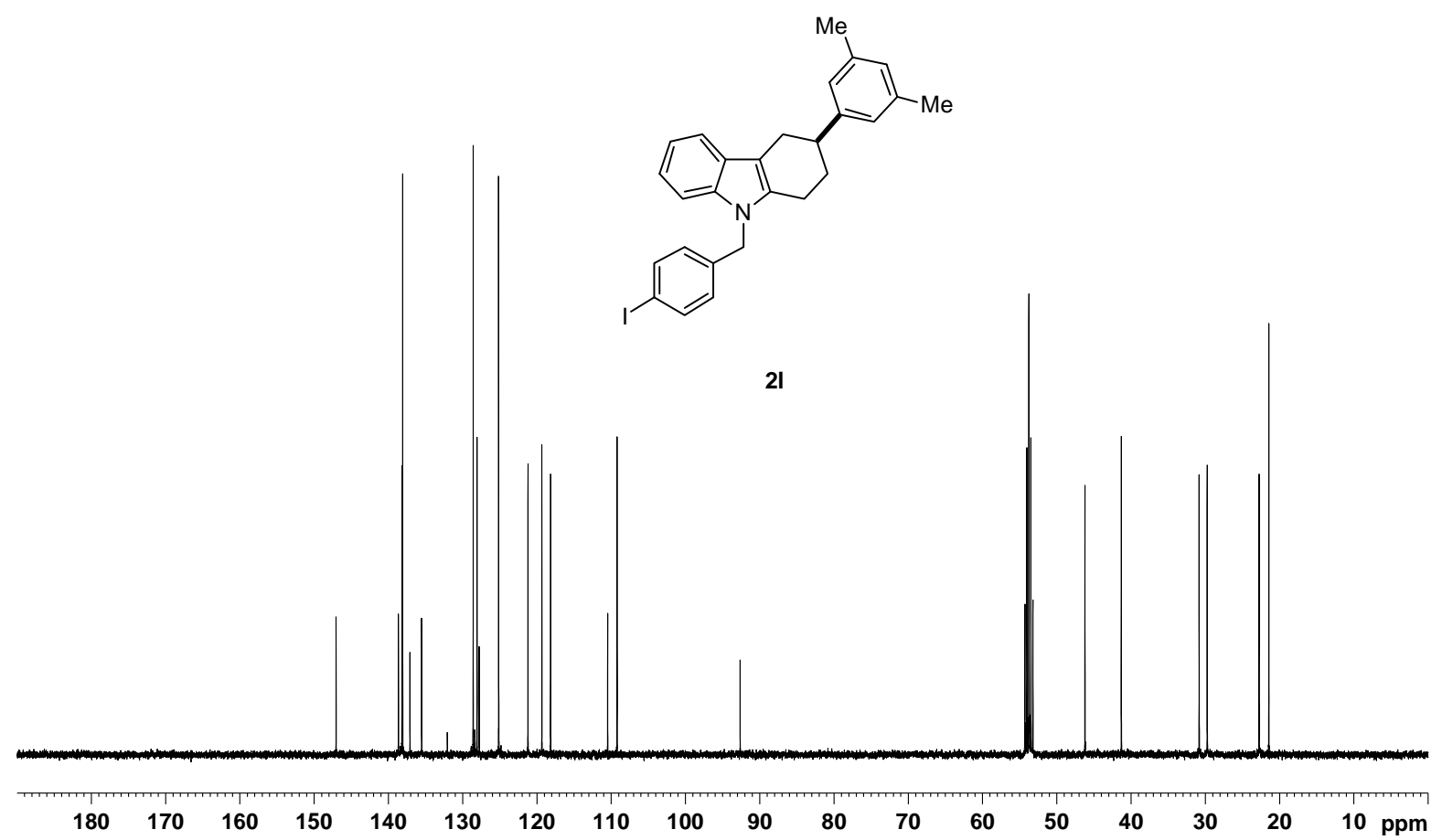



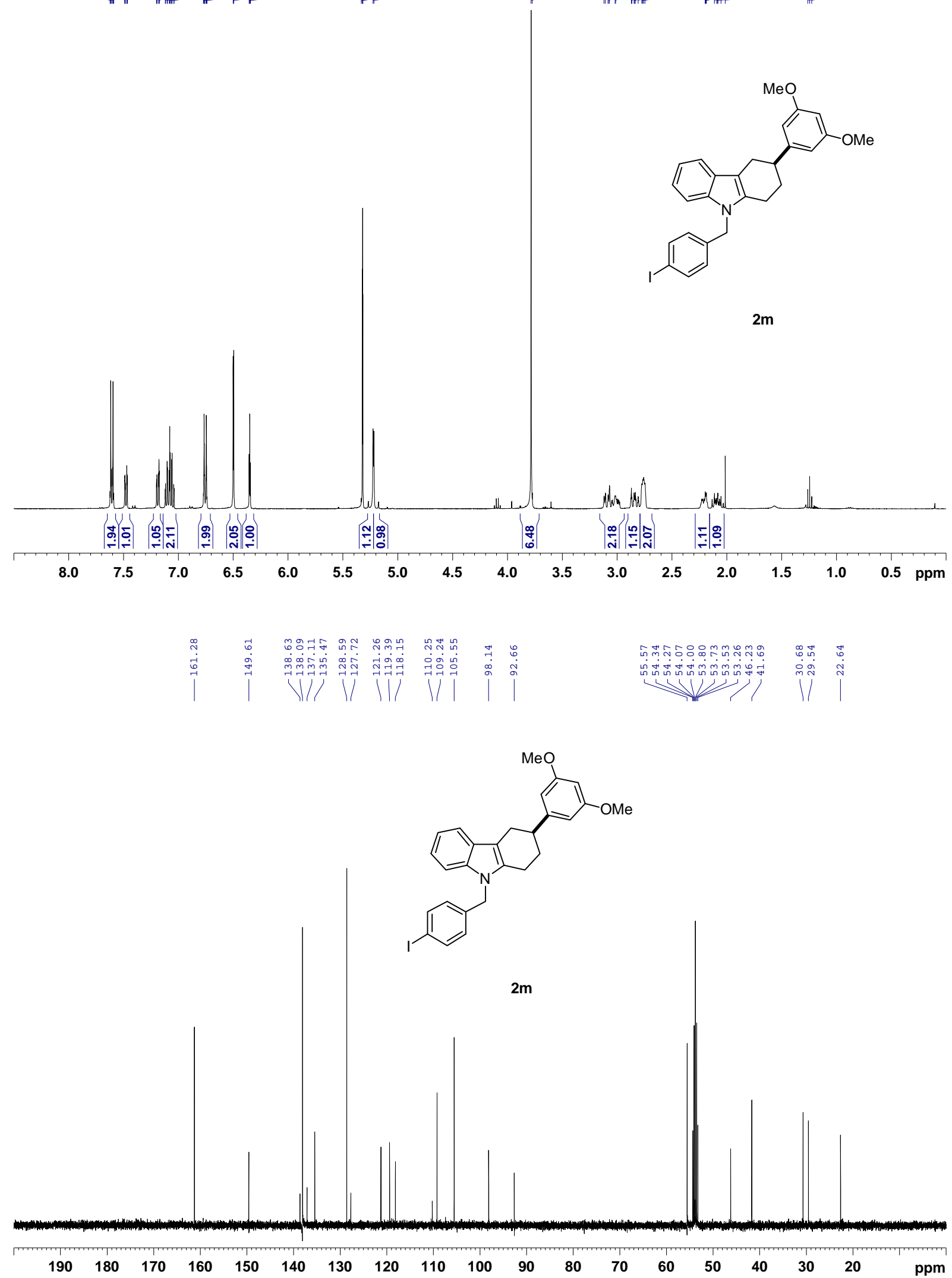

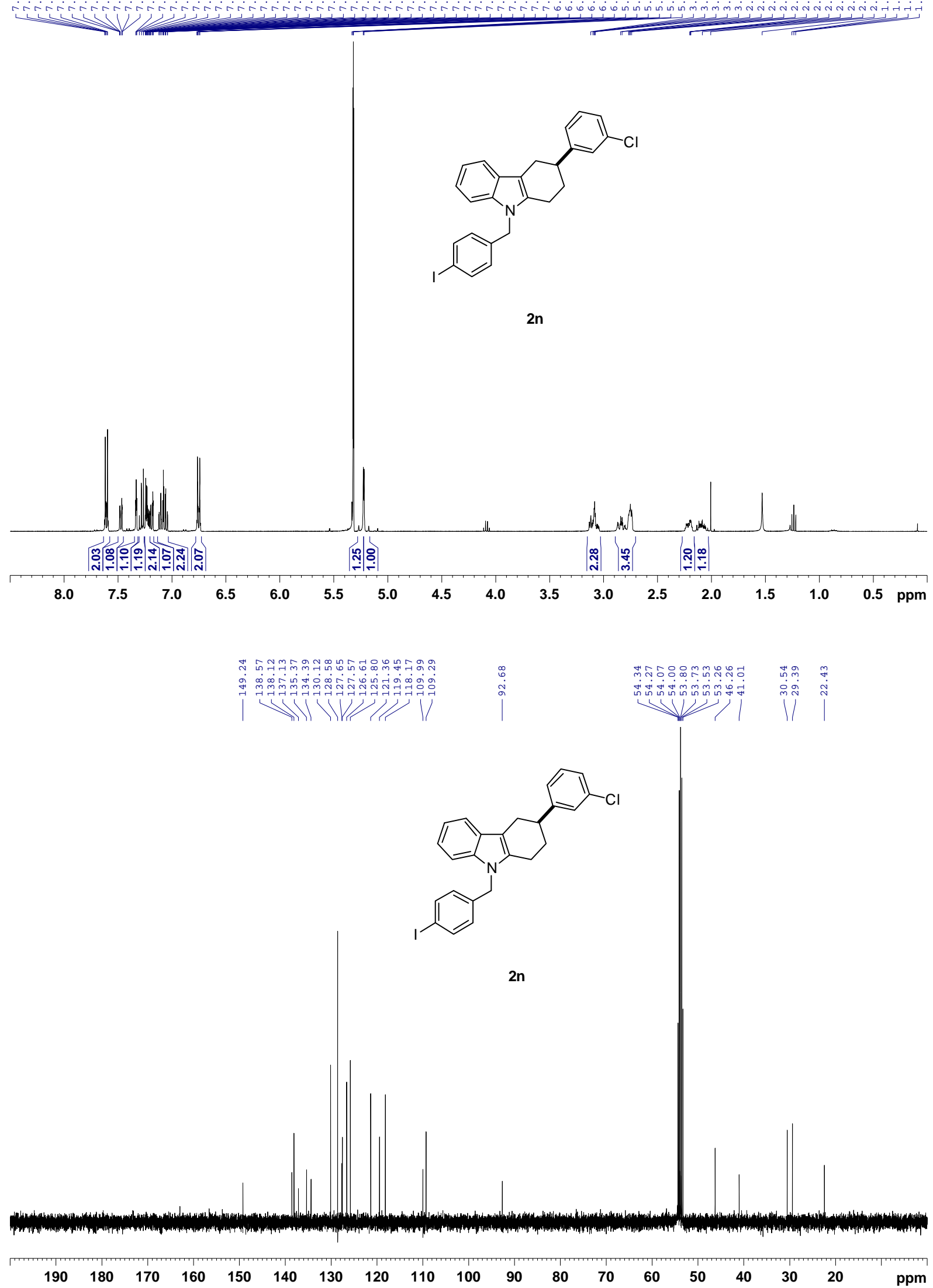

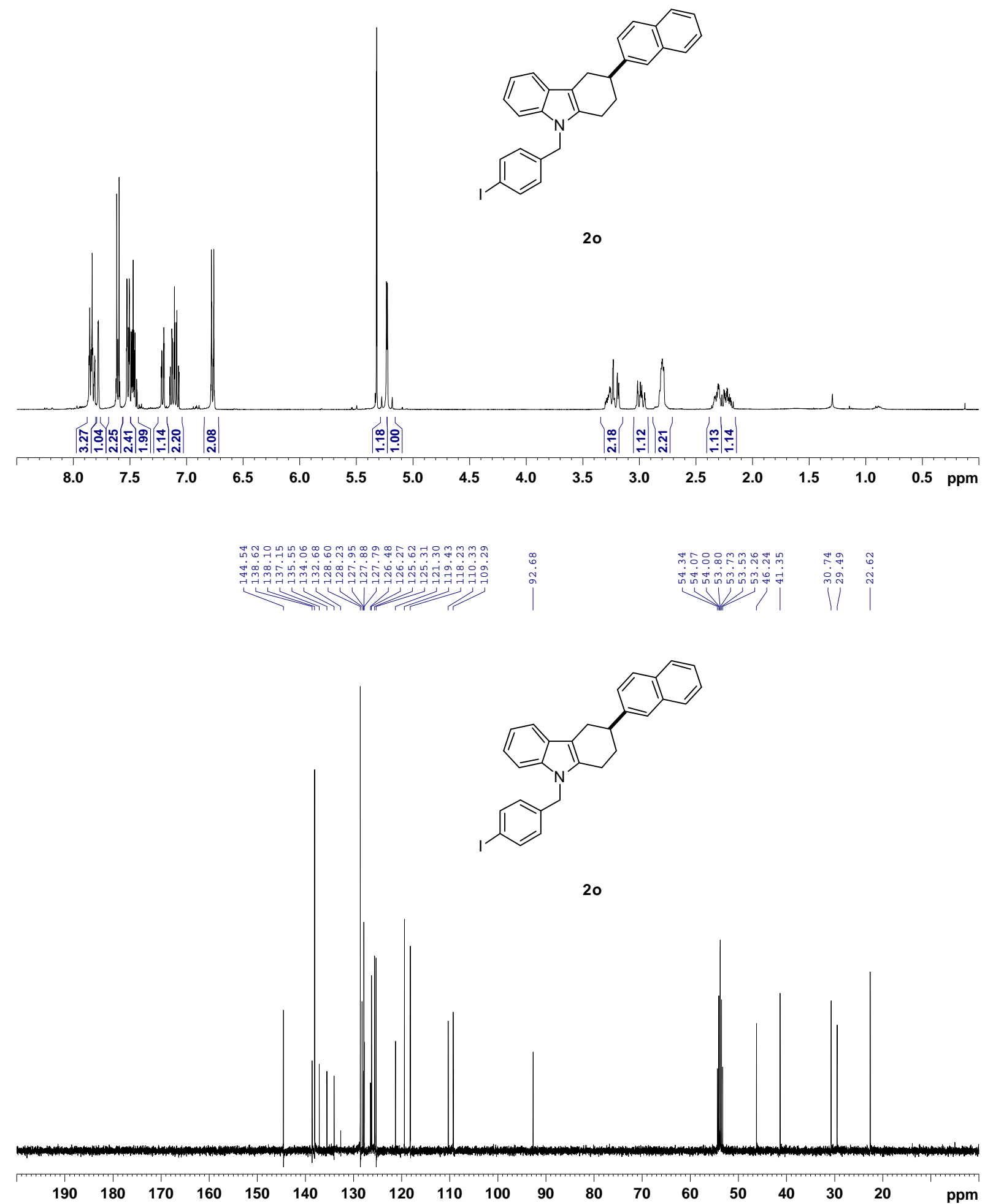


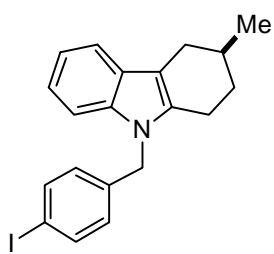

$2 p$

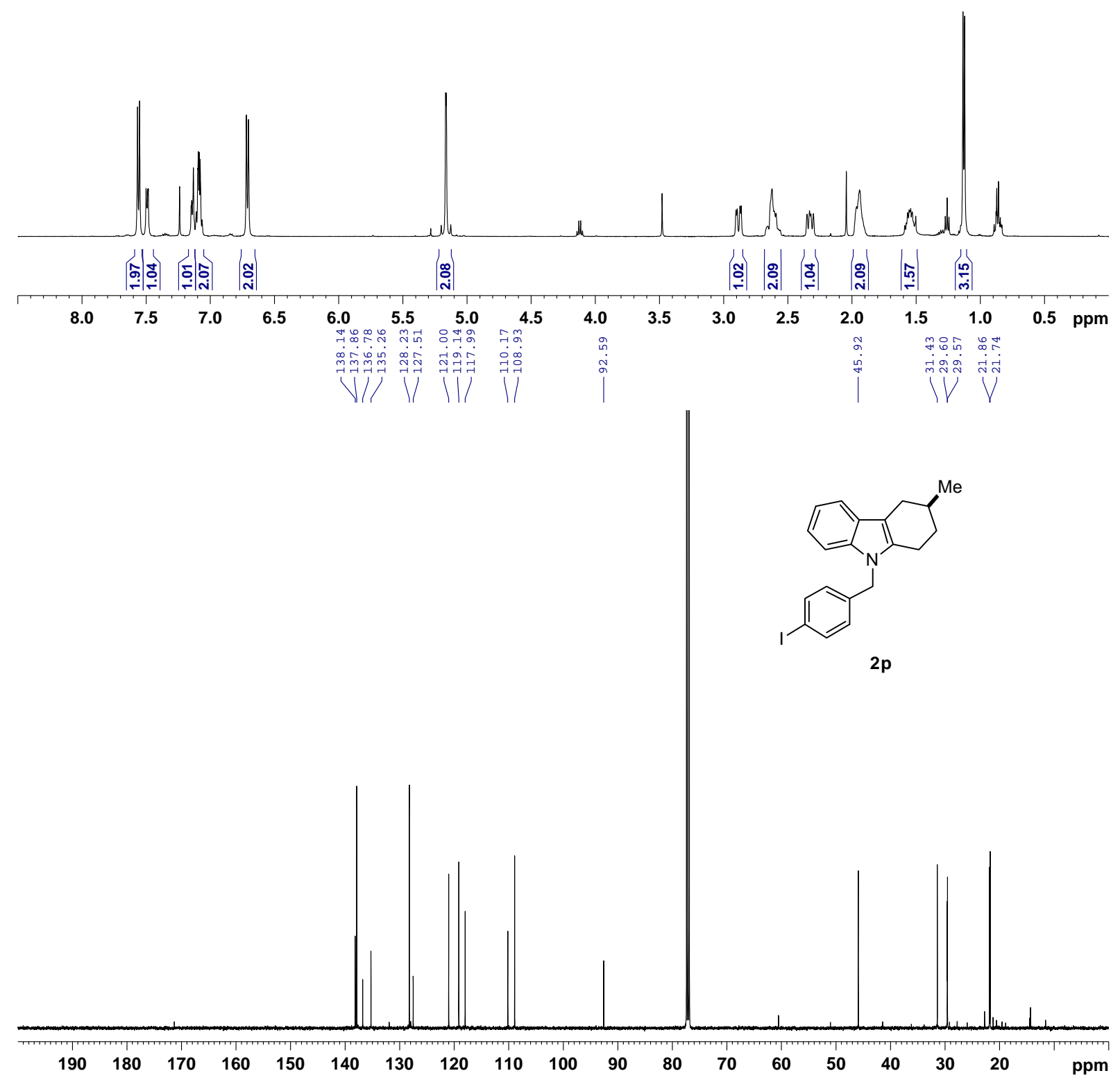



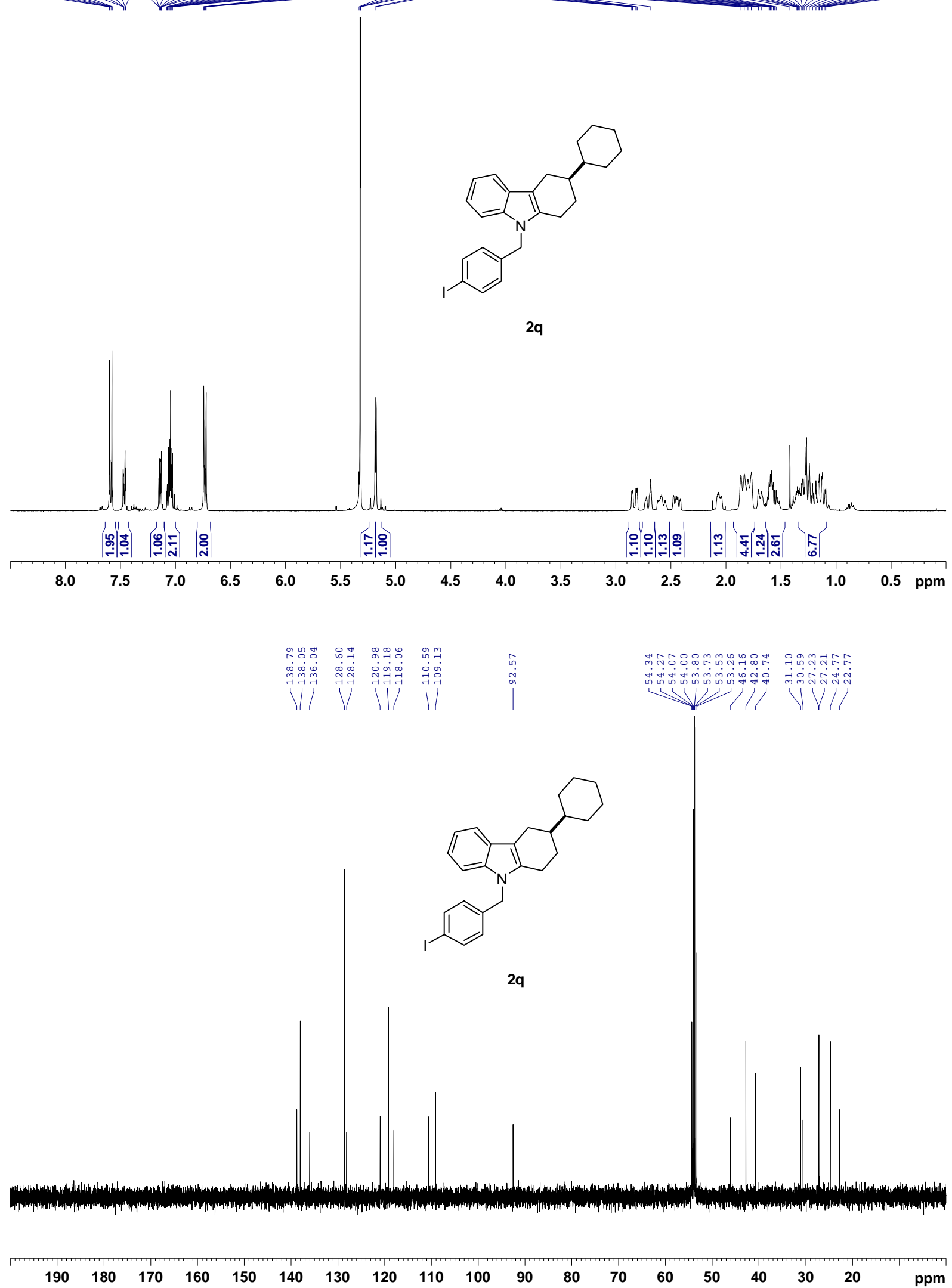


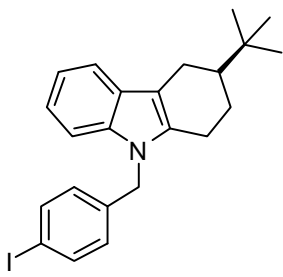

$2 r$
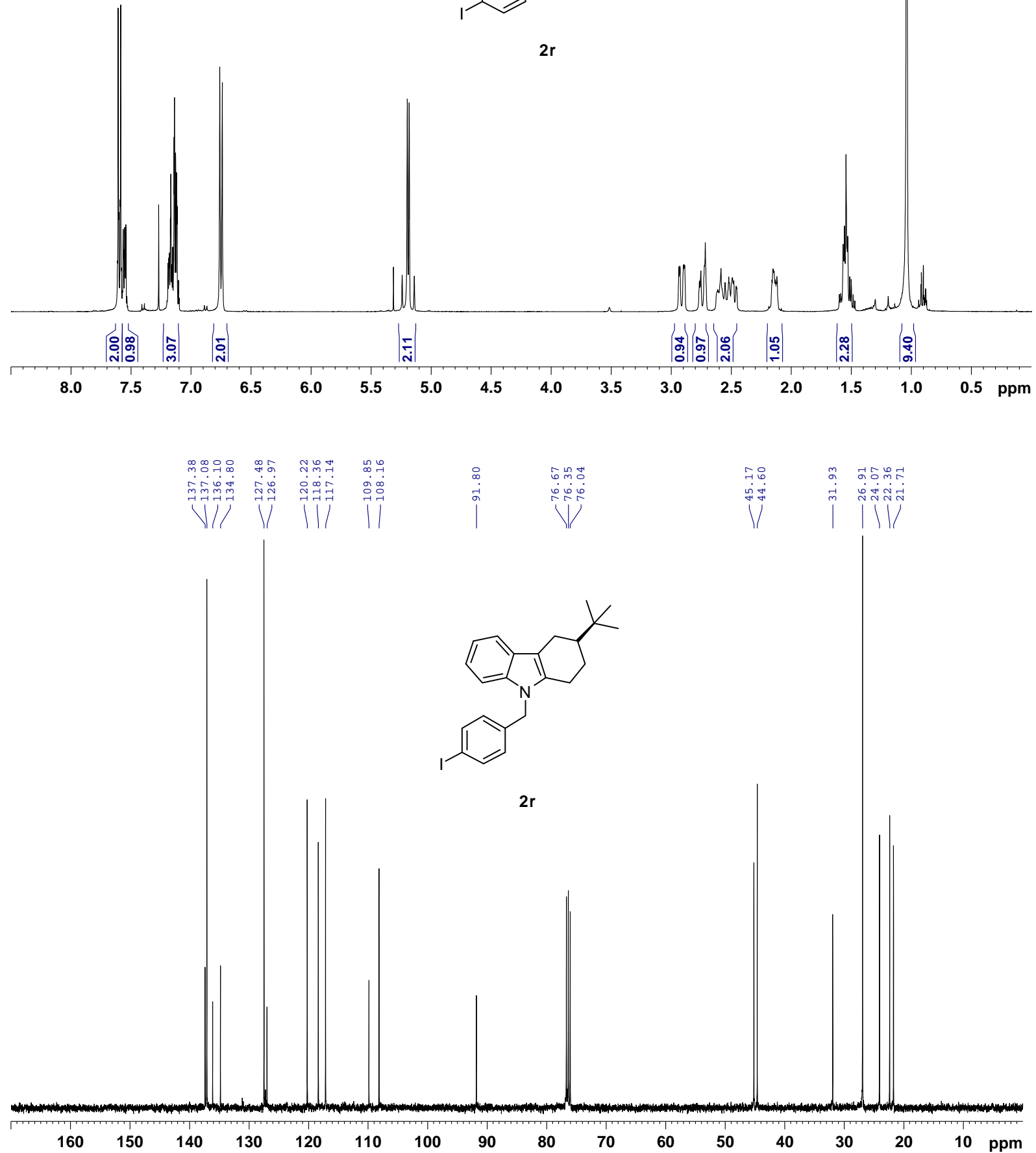


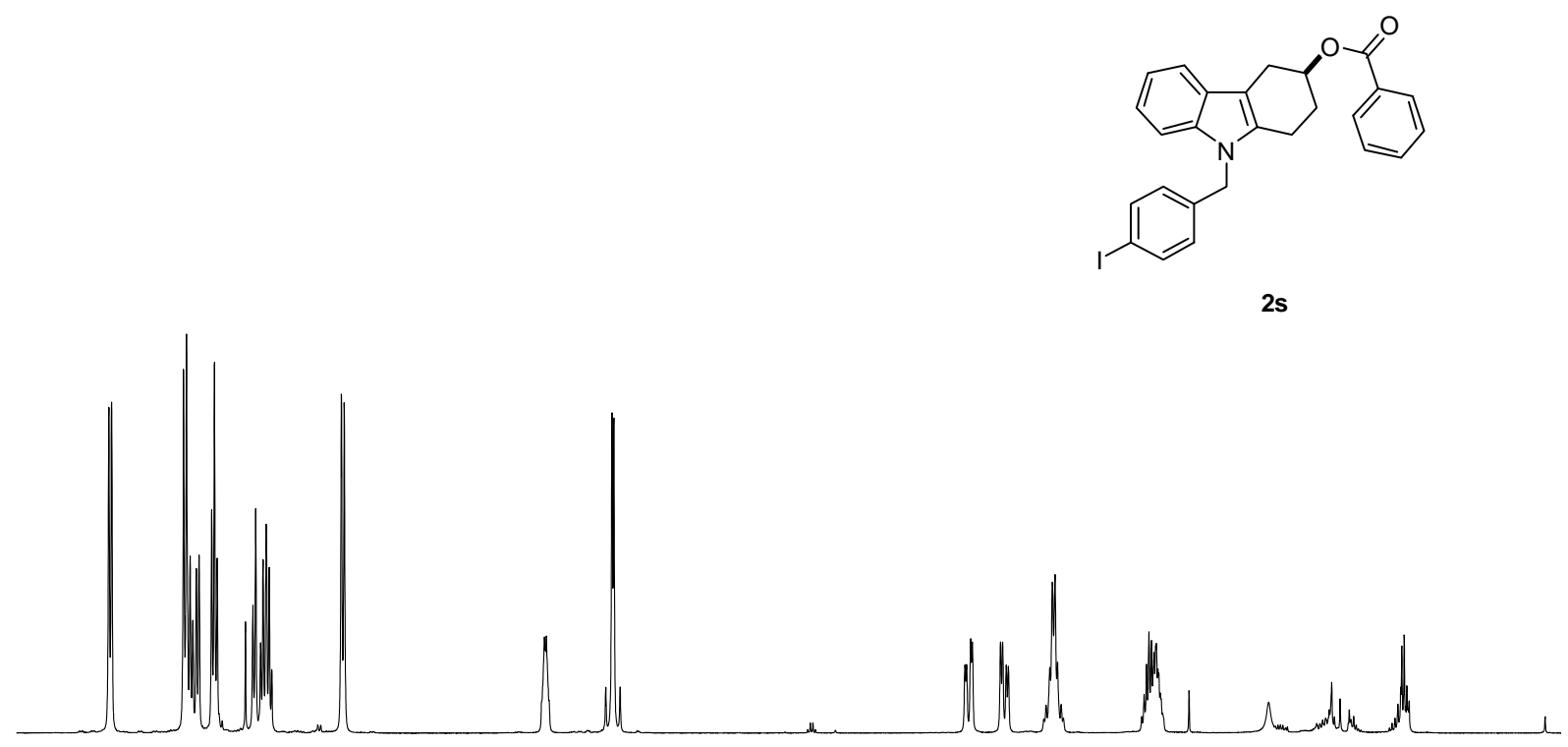

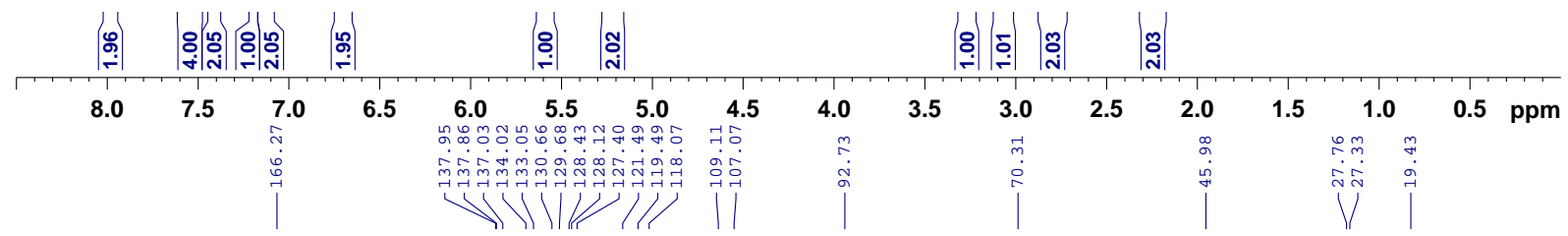

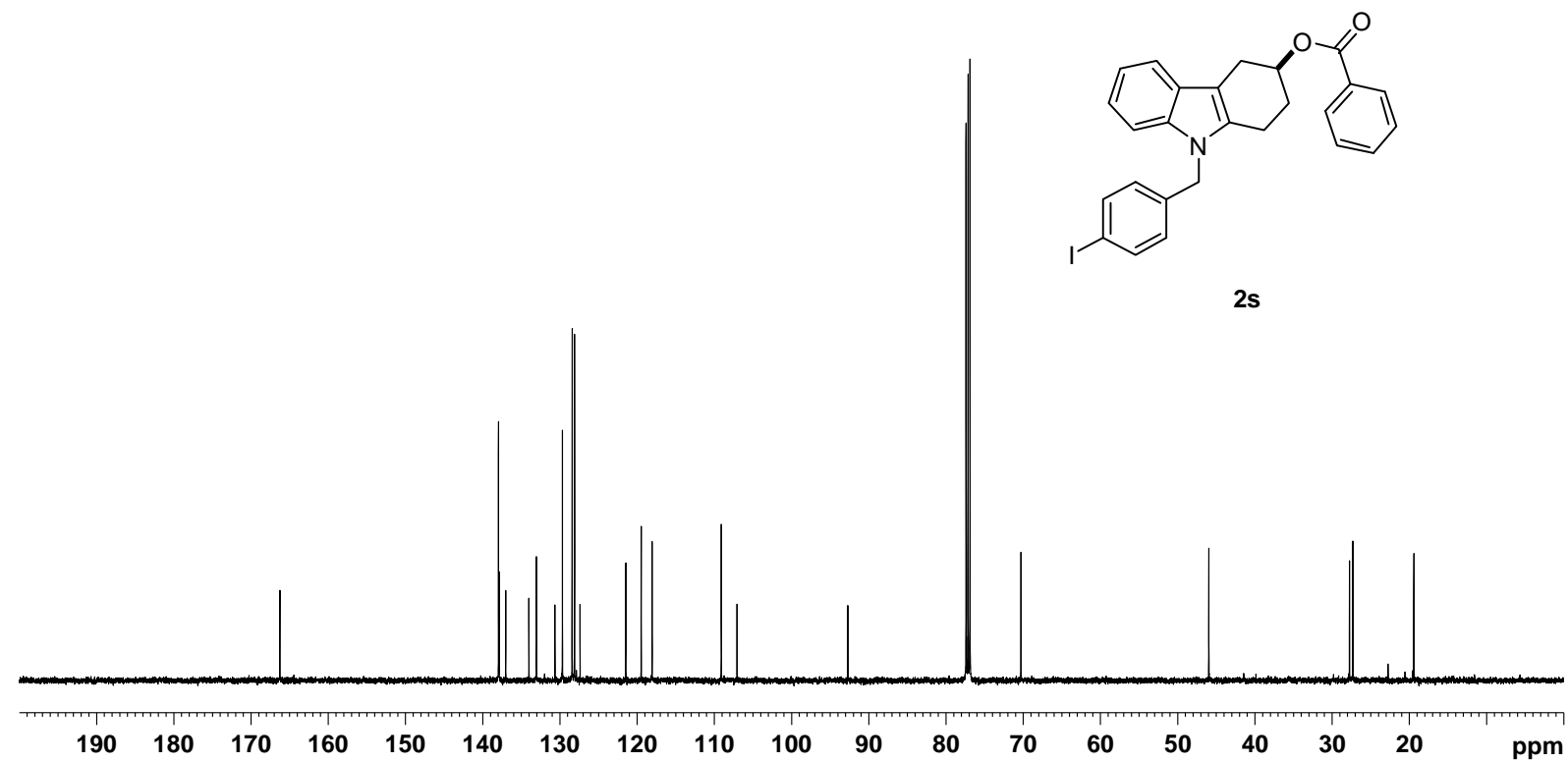




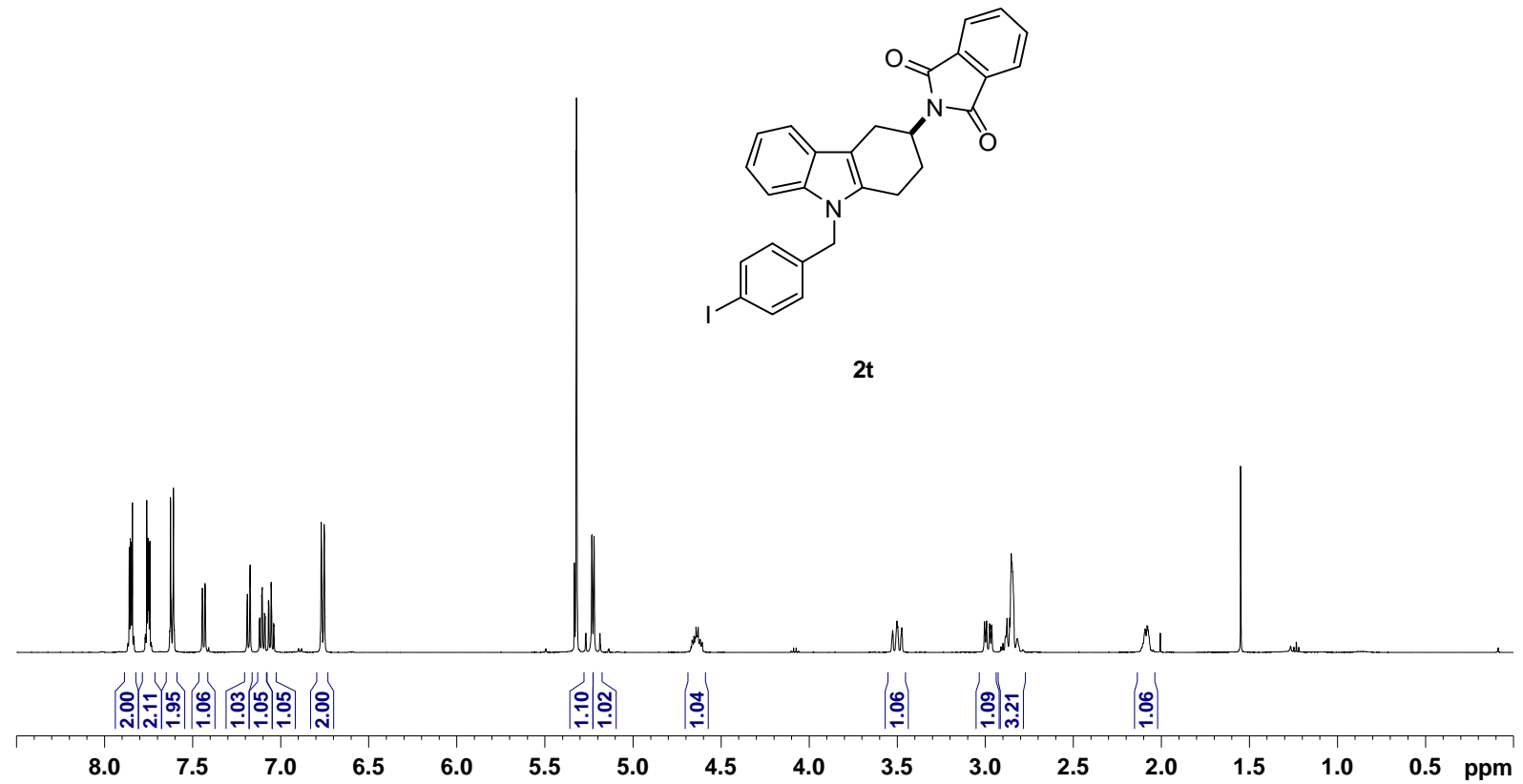

$2 t$

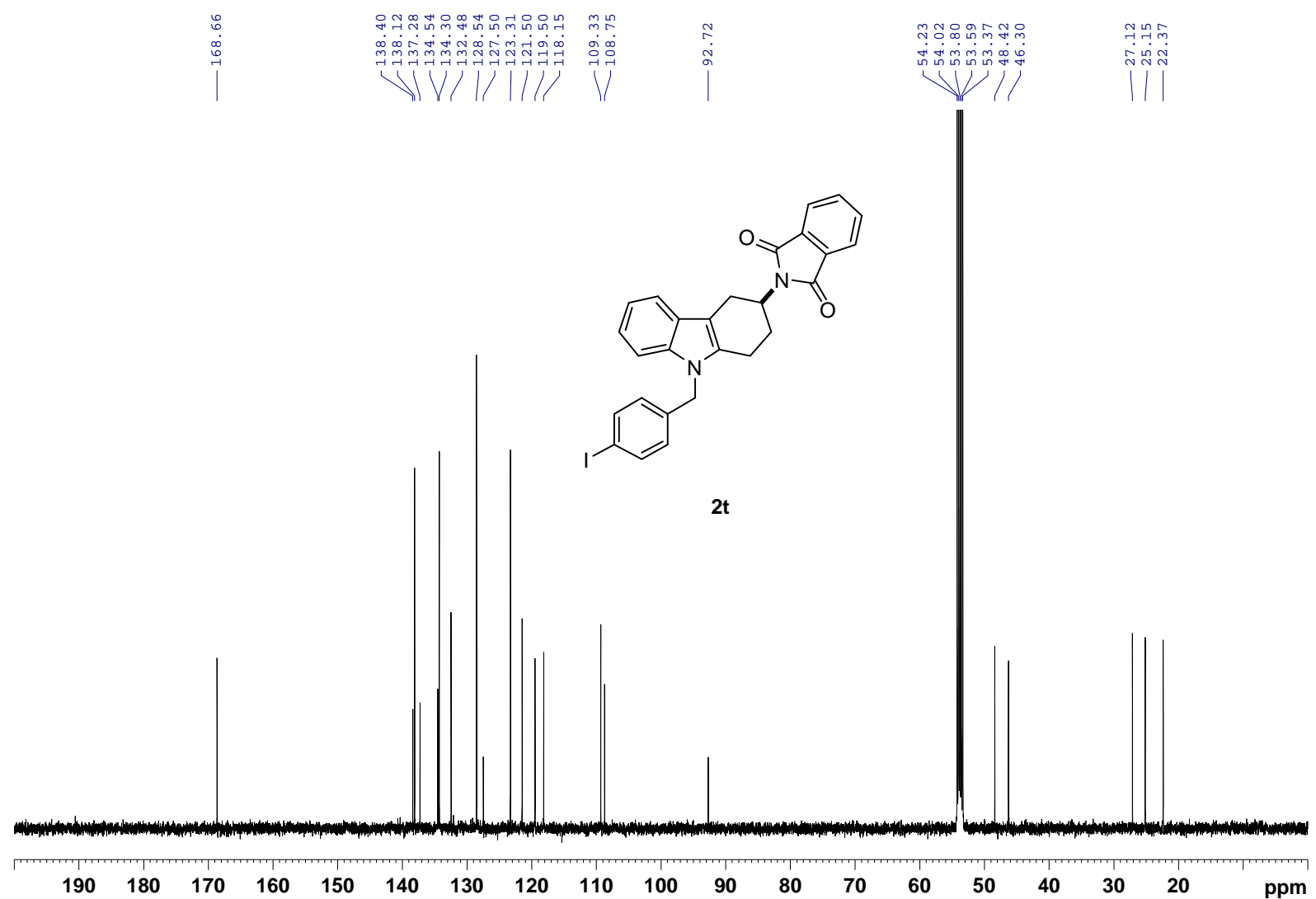


<smiles>CC1(c2ccccc2)CCc2c(c3ccccc3n2Cc2ccc(I)cc2)C1</smiles>

$2 u$

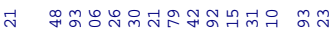

๑

|<smiles>CC1(c2ccccc2)CCc2c(c3ccccc3n2Cc2ccc(I)cc2)C1</smiles>

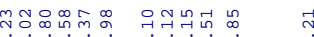

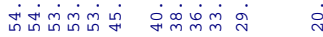

W $|1|||$

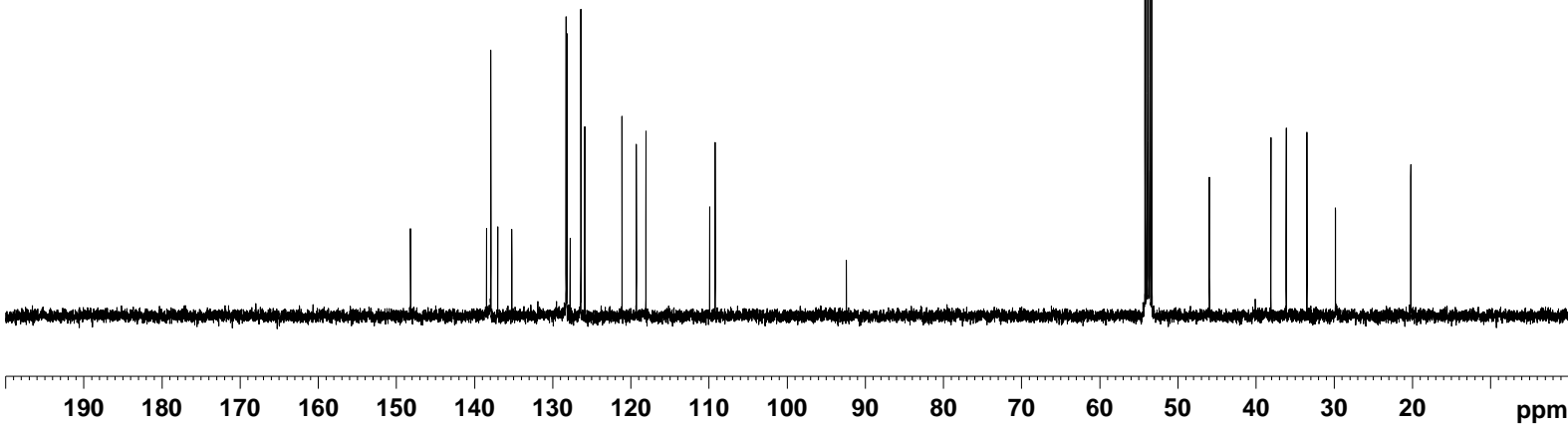



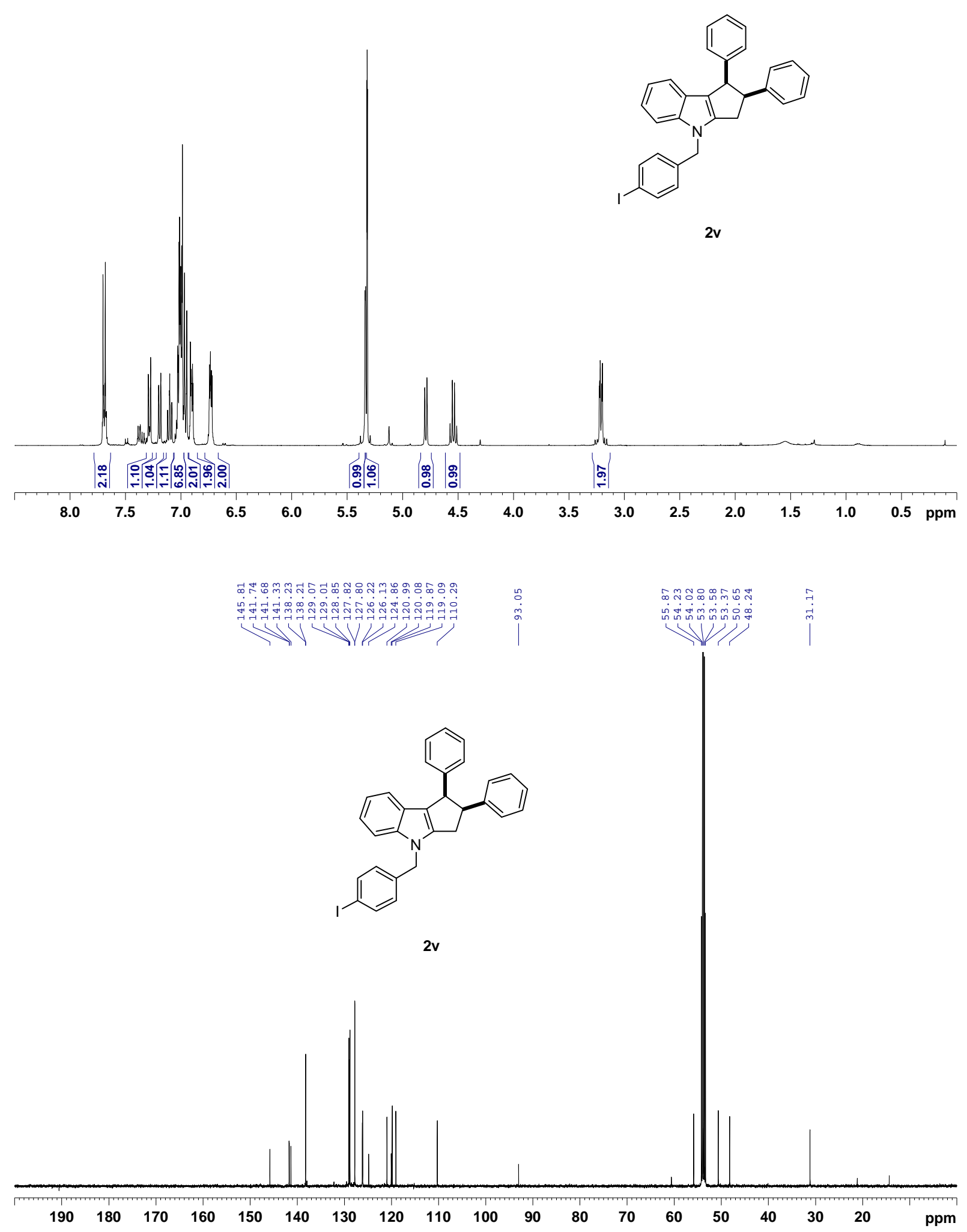


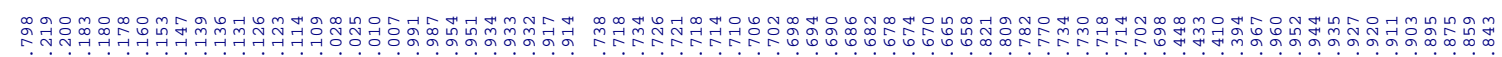

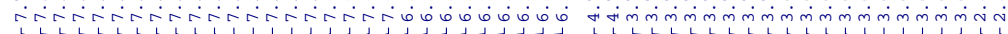

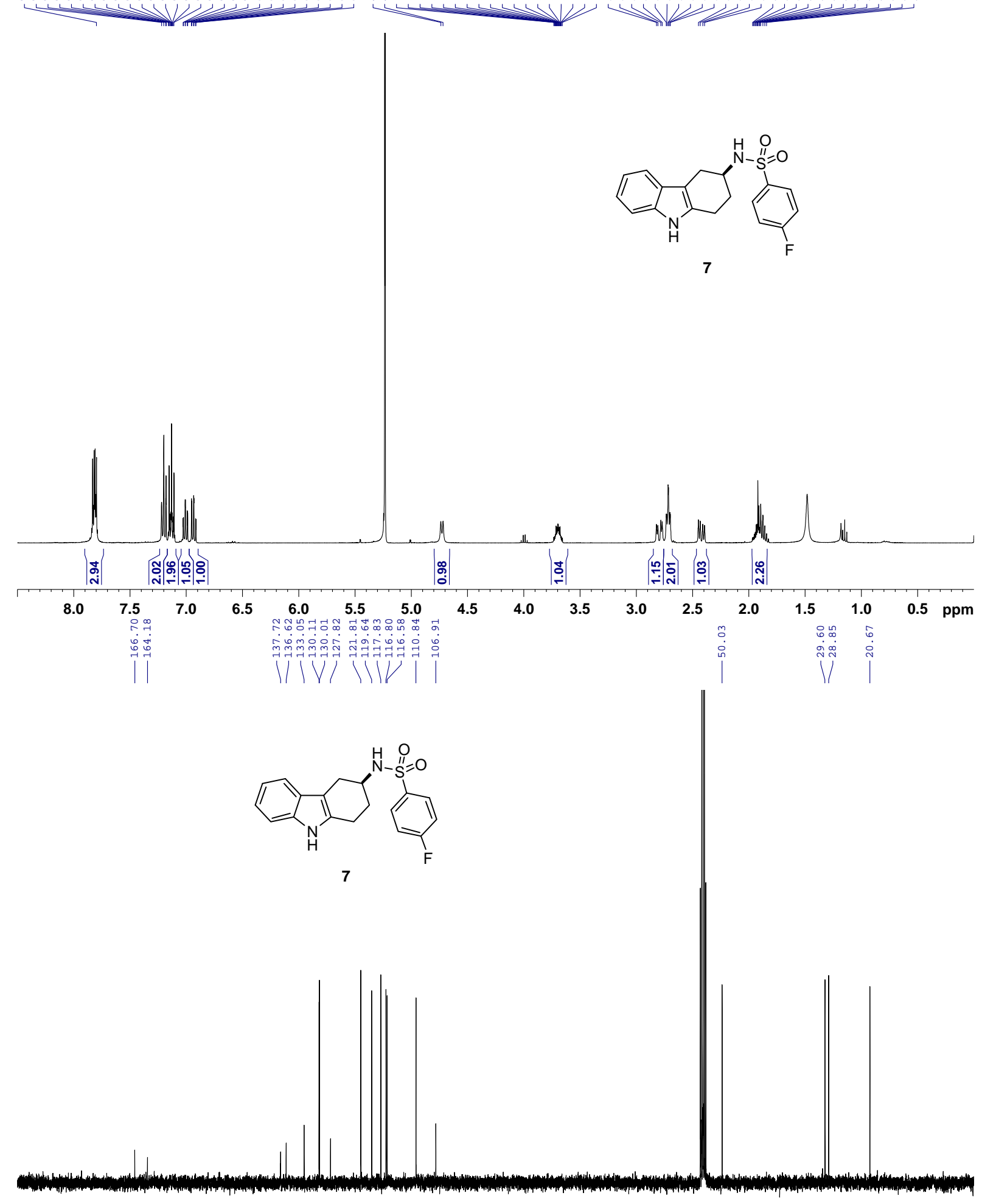

$\begin{array}{lllllllllllllllllll}180 & 170 & 160 & 150 & 140 & 130 & 120 & 110 & 100 & 90 & 80 & 70 & 60 & 50 & 40 & 30 & 20 & 10 & \mathrm{ppm}\end{array}$ 
HPLC traces

mAU
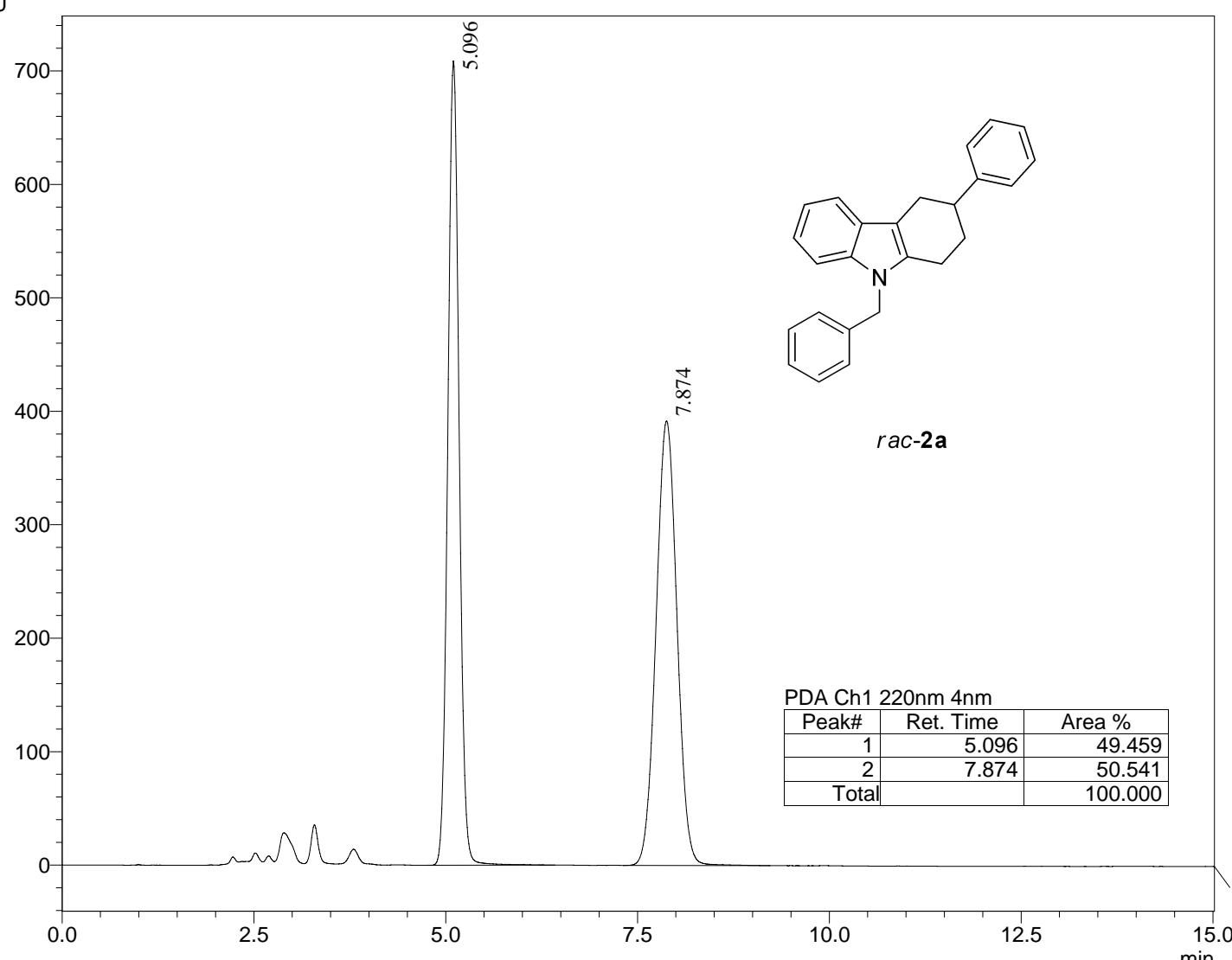

1PDA Multi 1

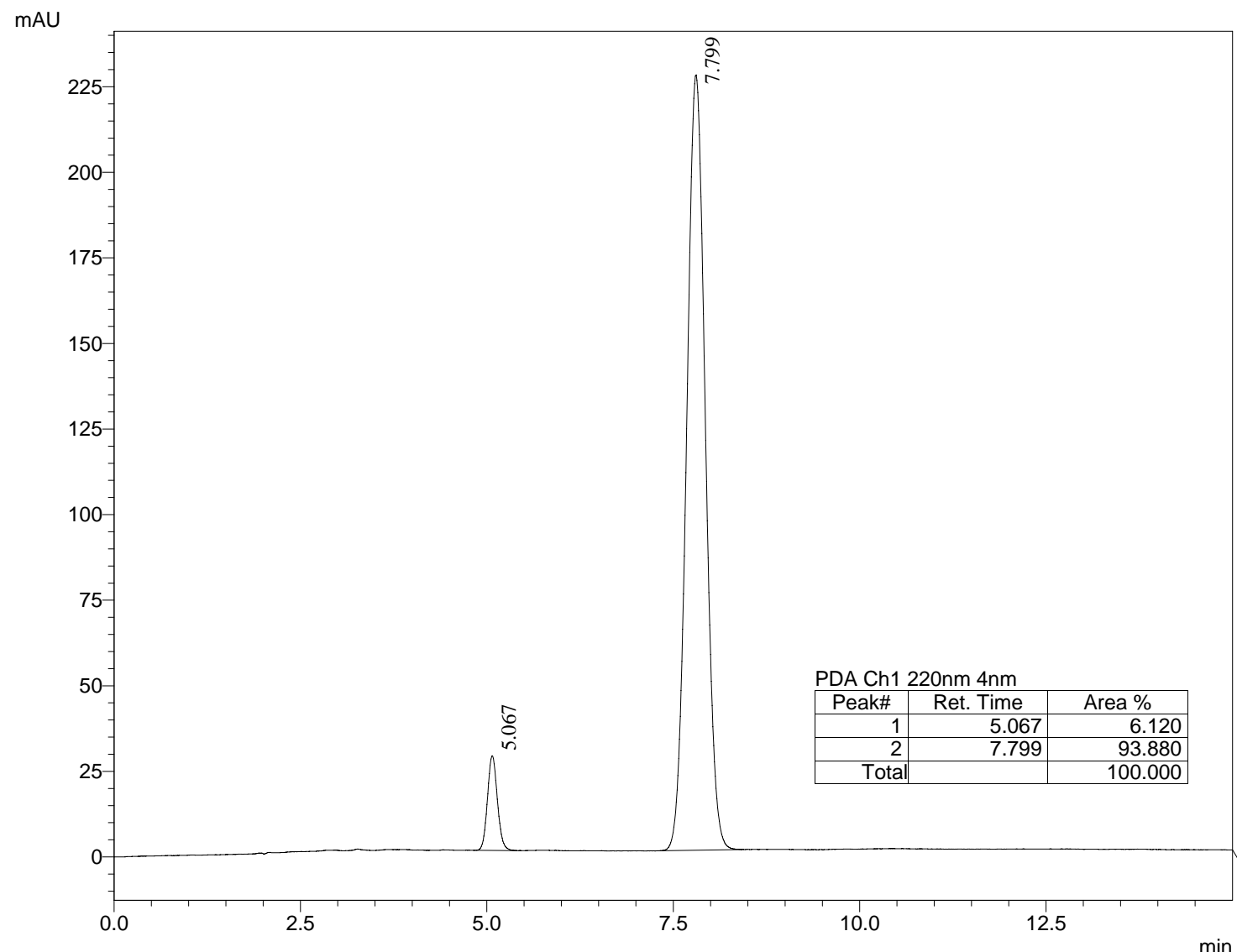




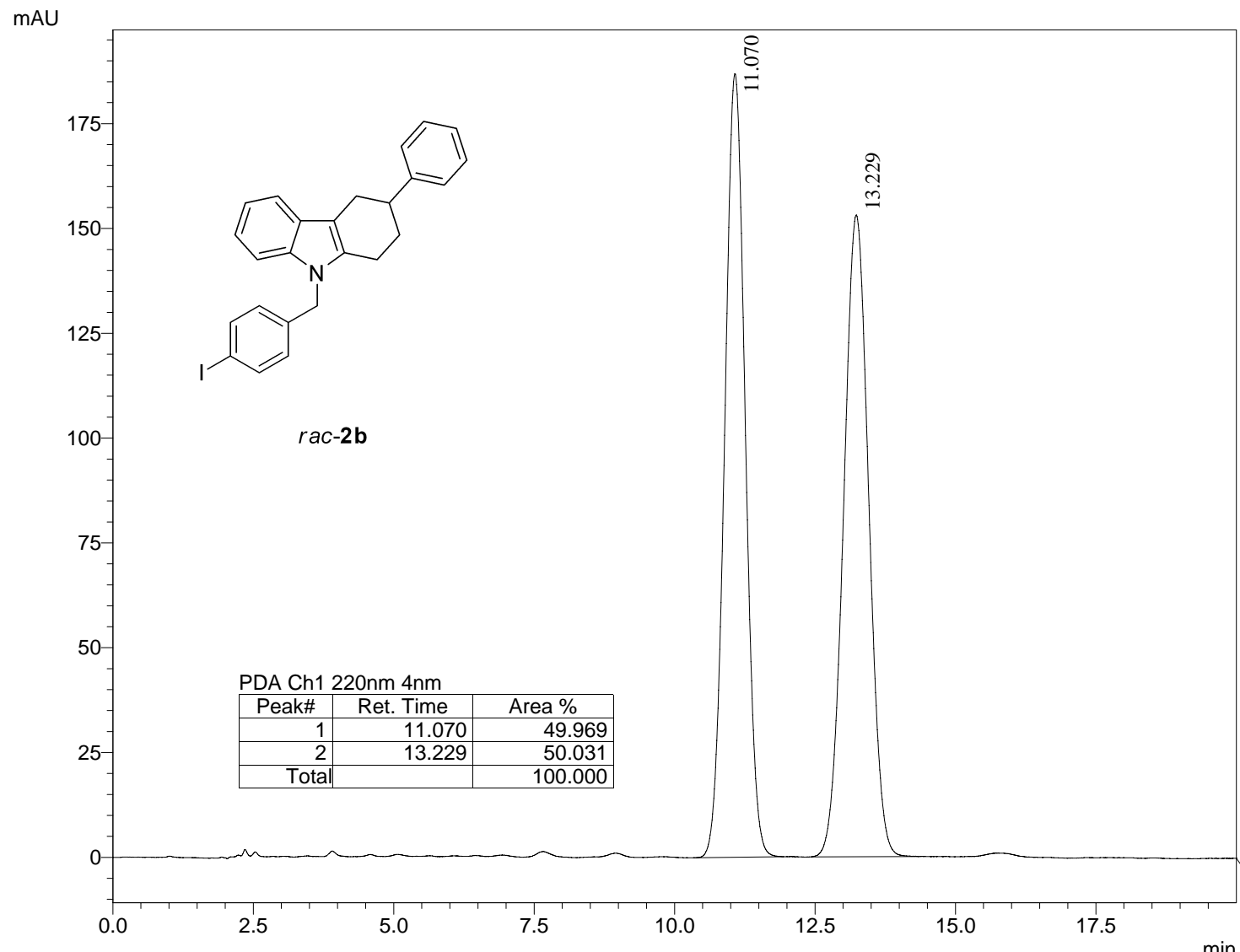

1PDA Multi 1

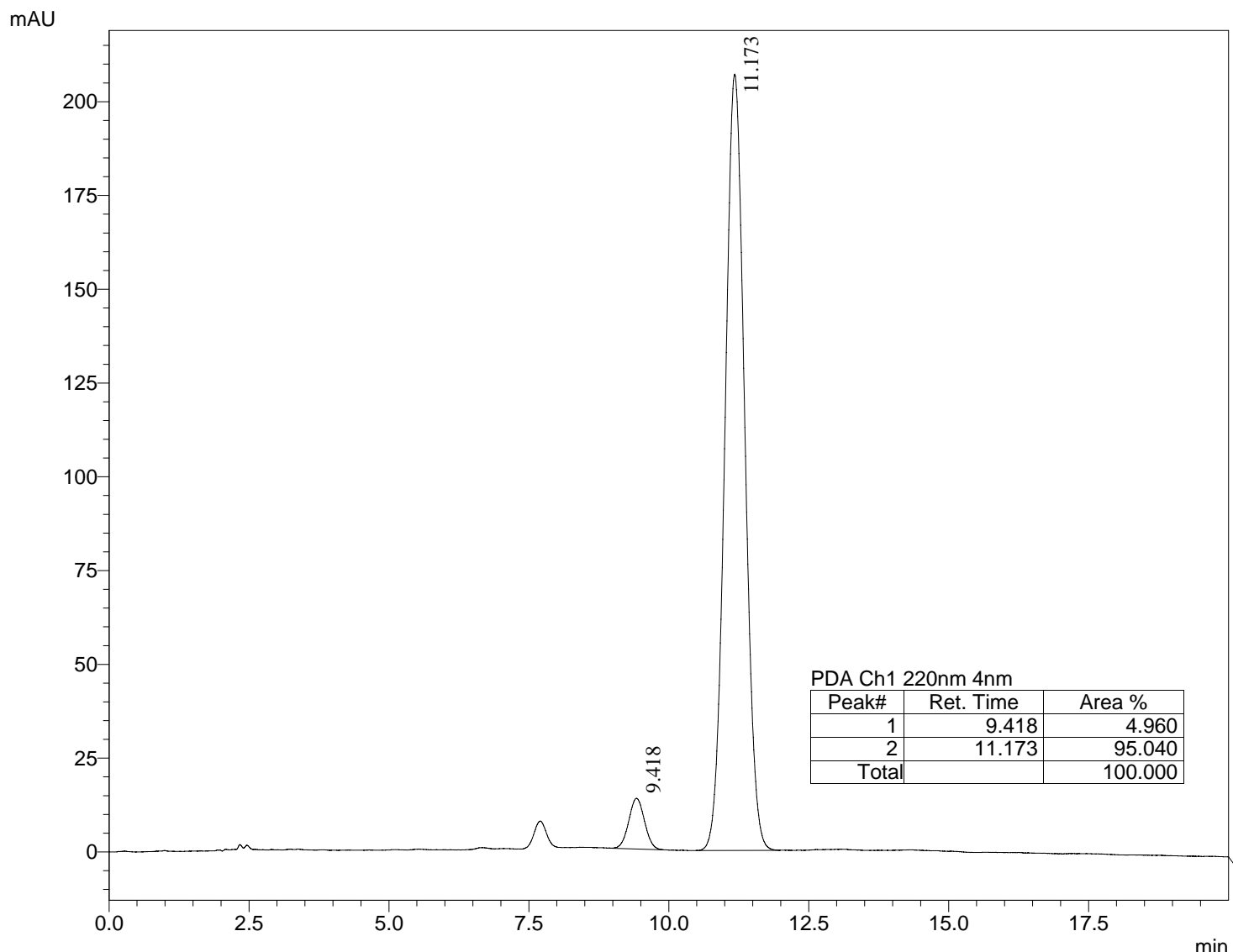


mAU

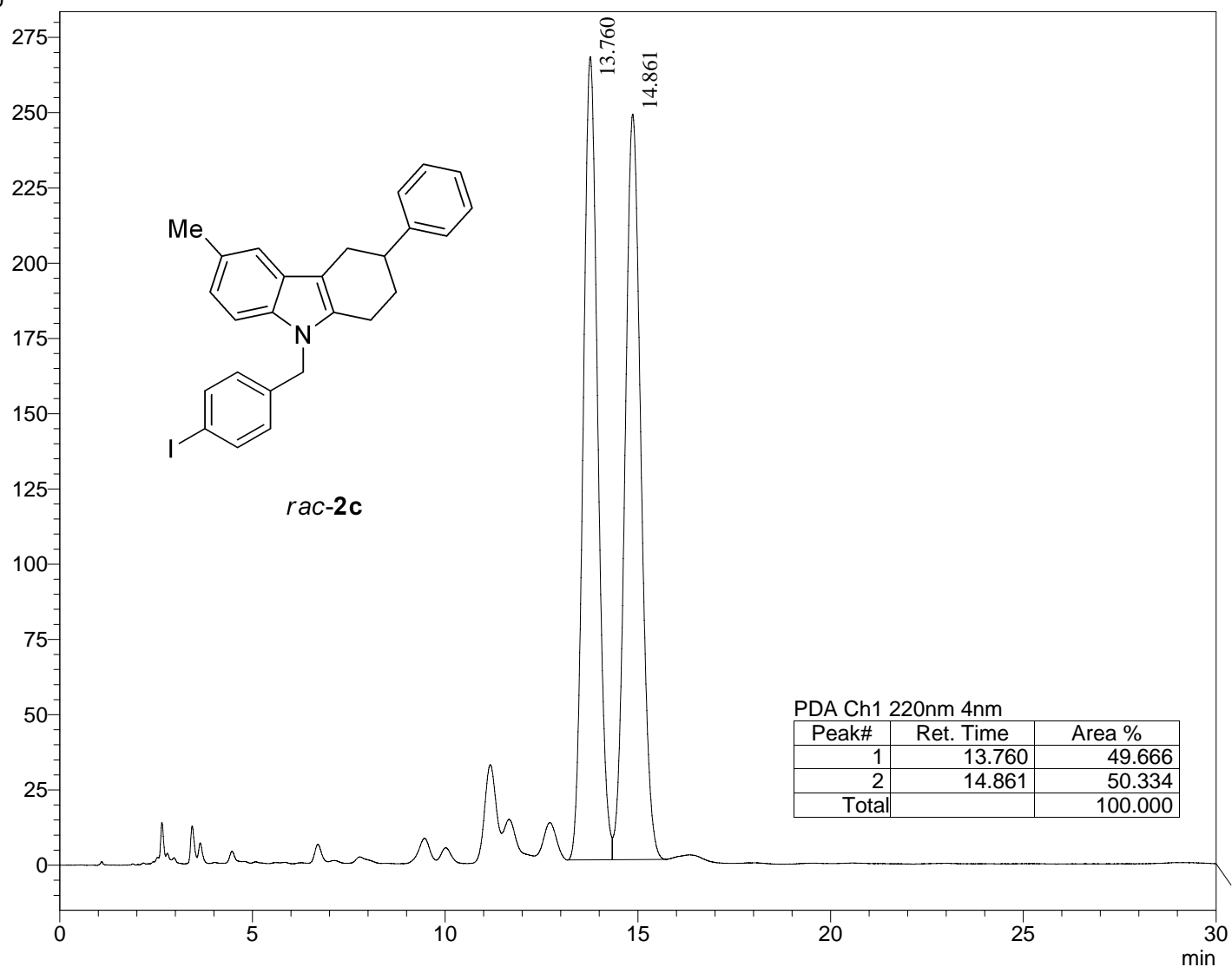

1PDA Multi 1

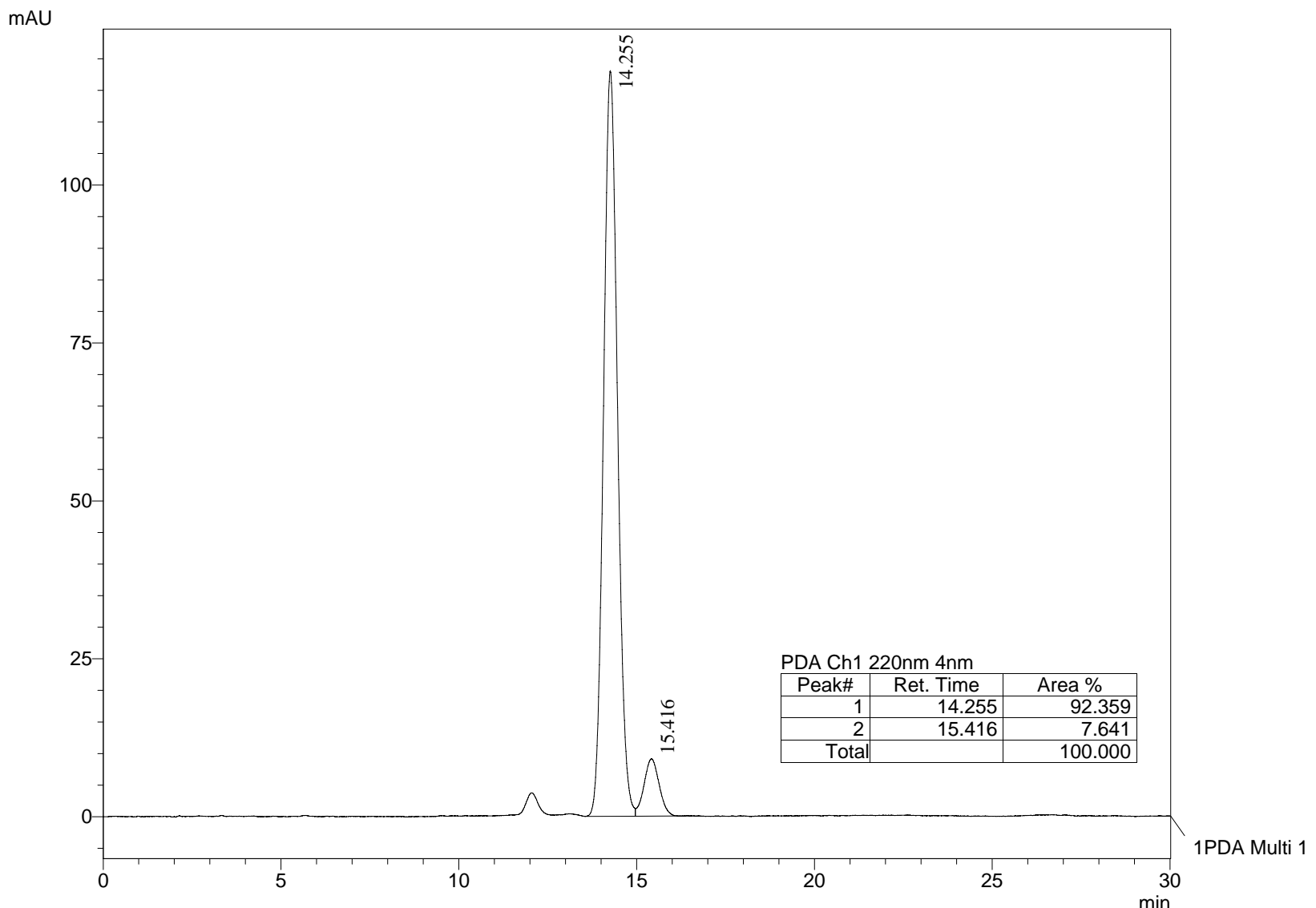




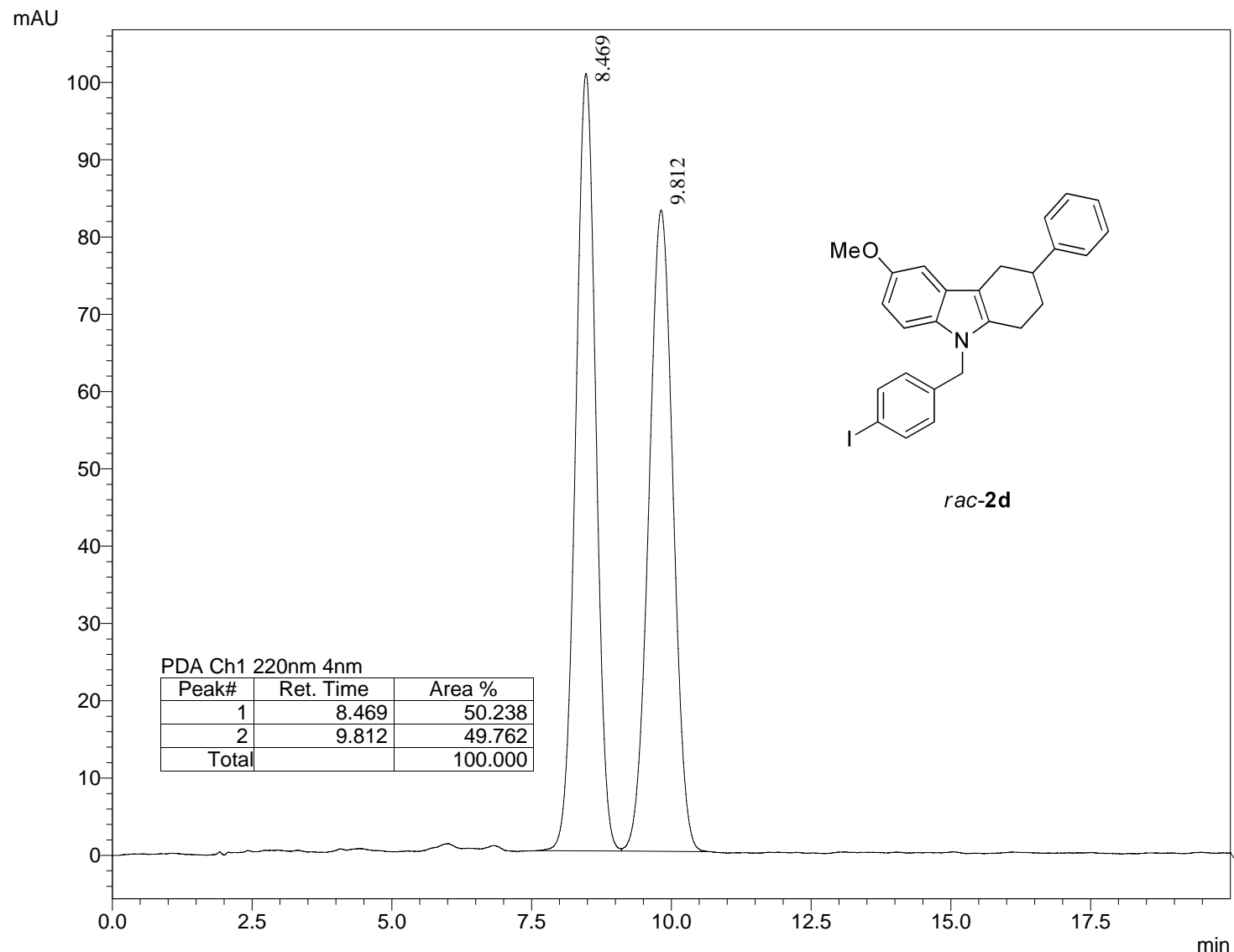

1PDA Multi 1

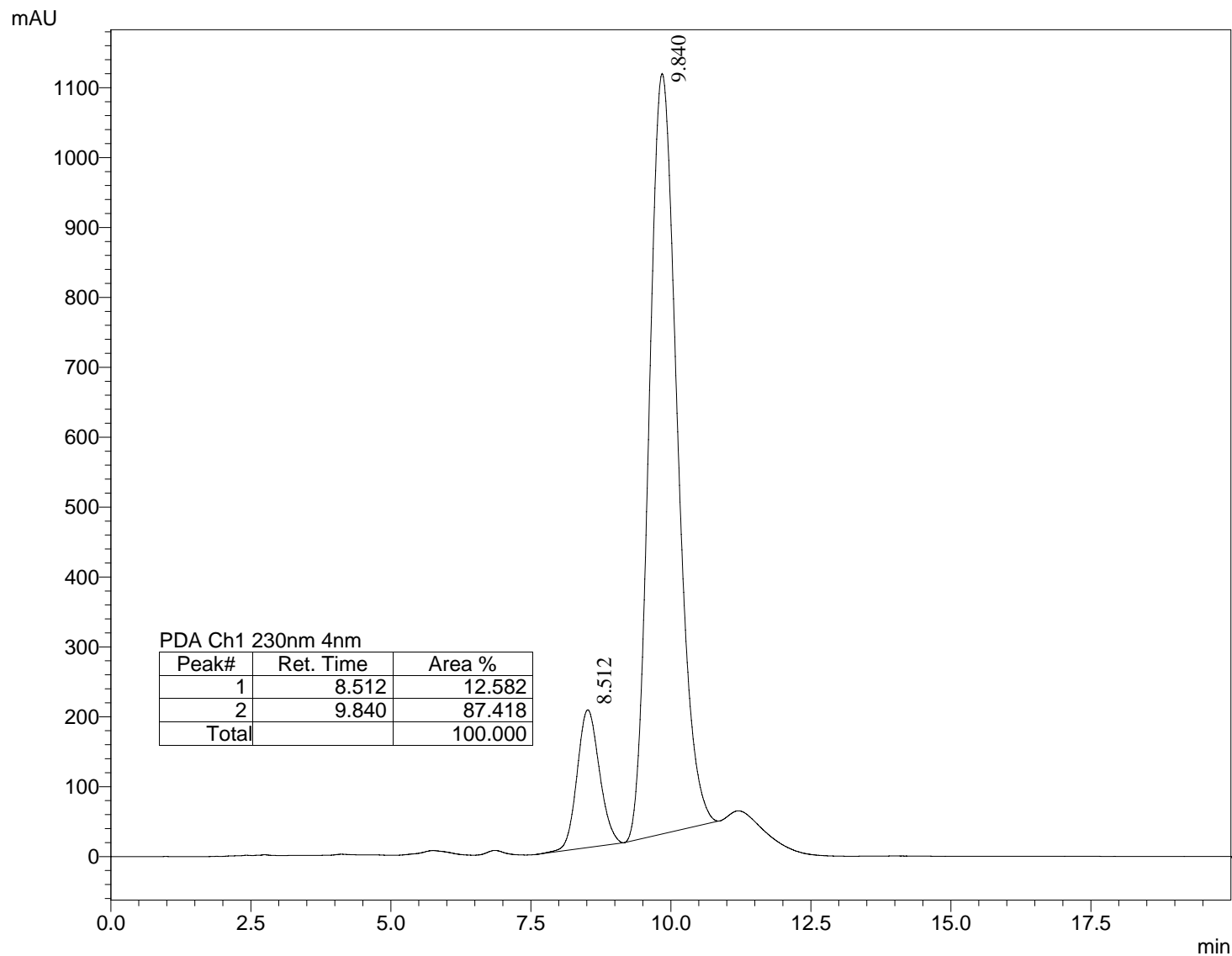




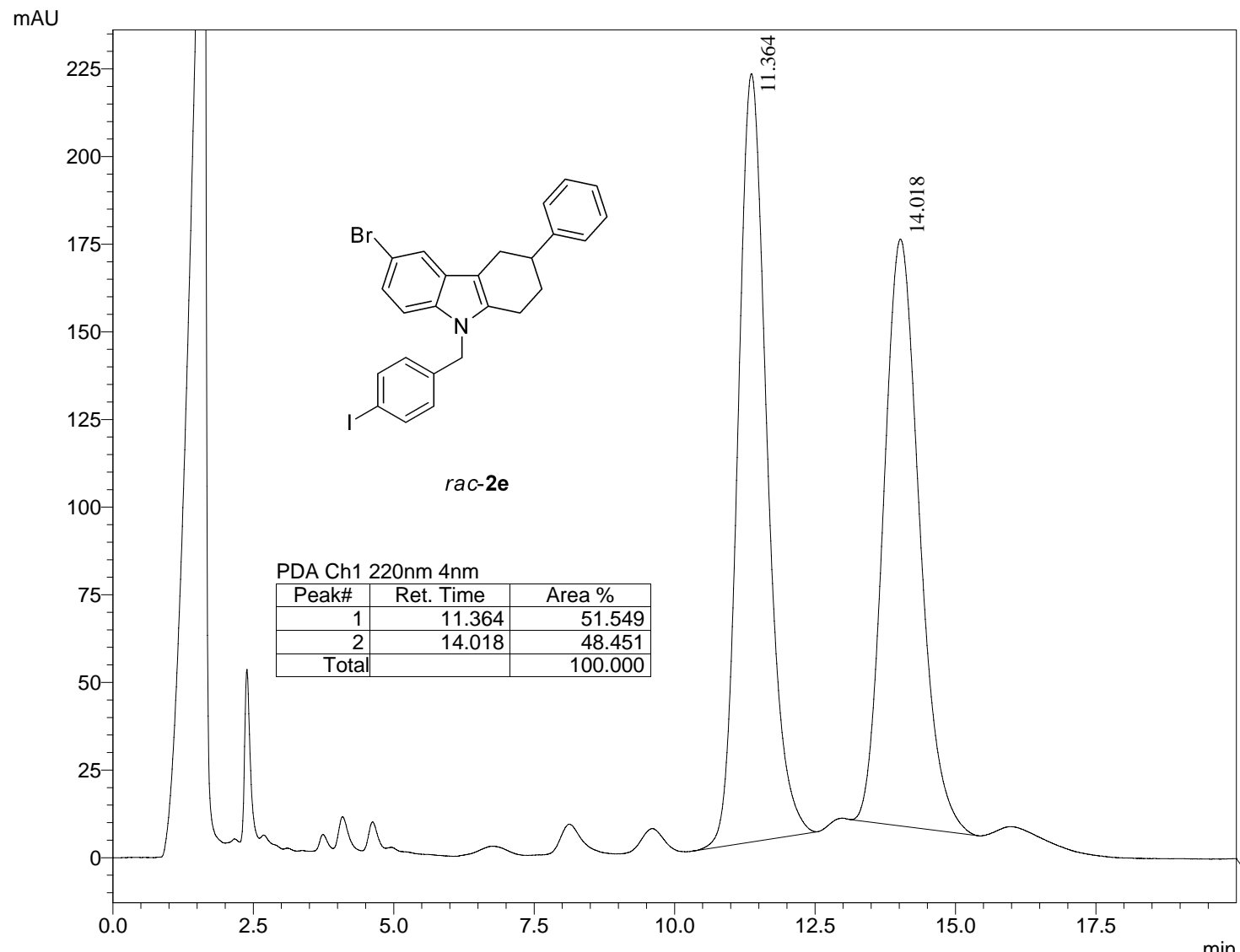

1PDA Multi 1

$\mathrm{mA}$

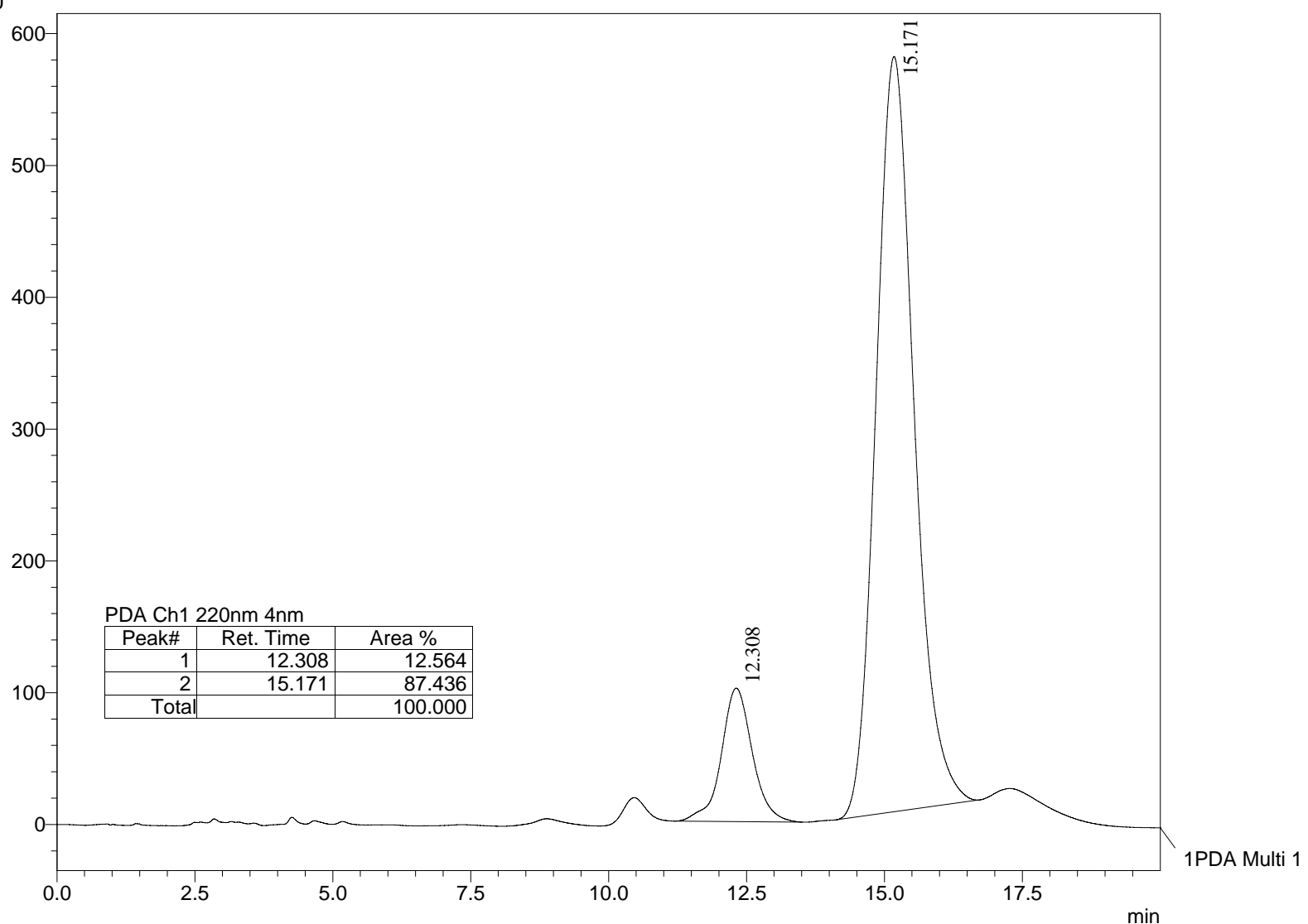




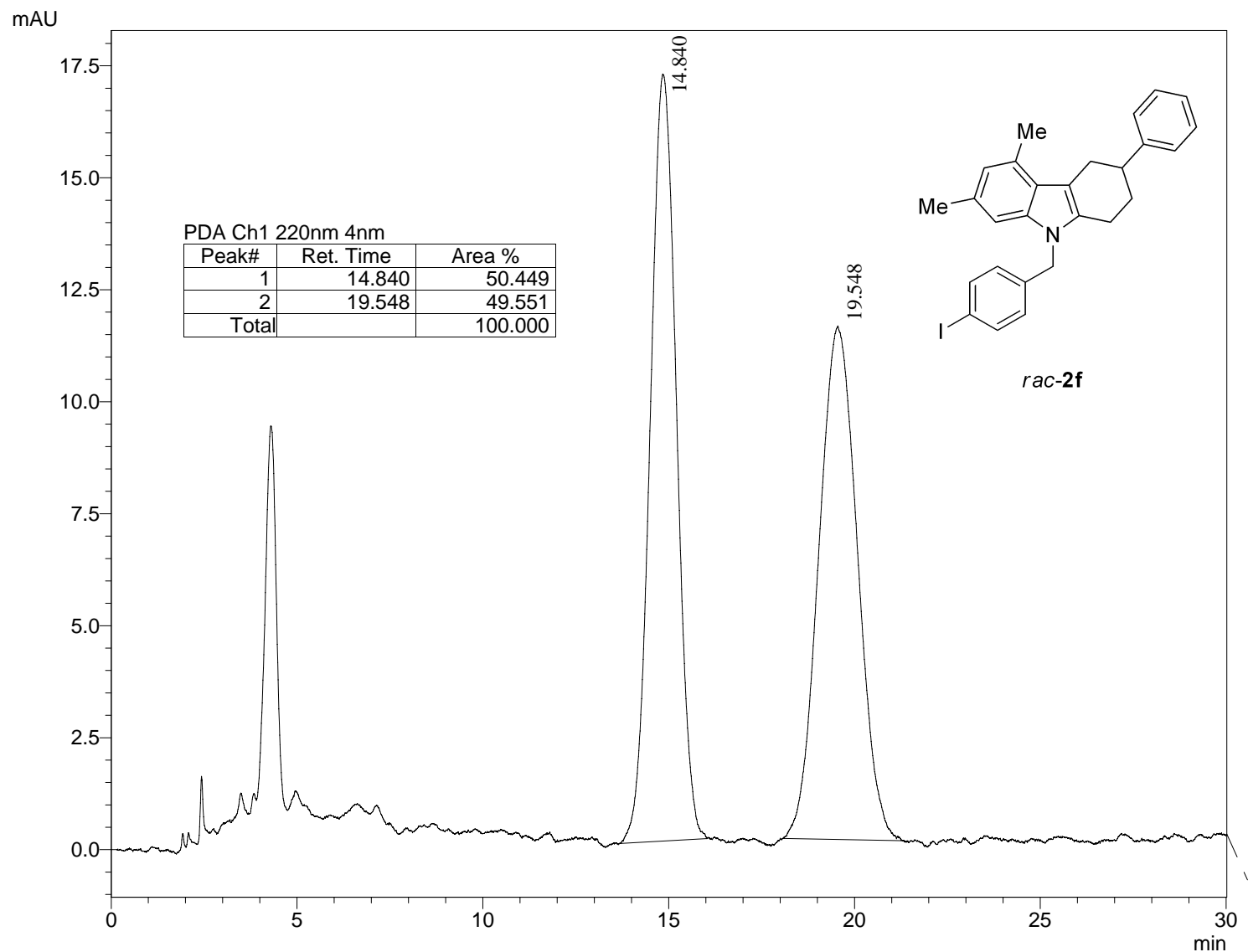

1PDA Multi 1

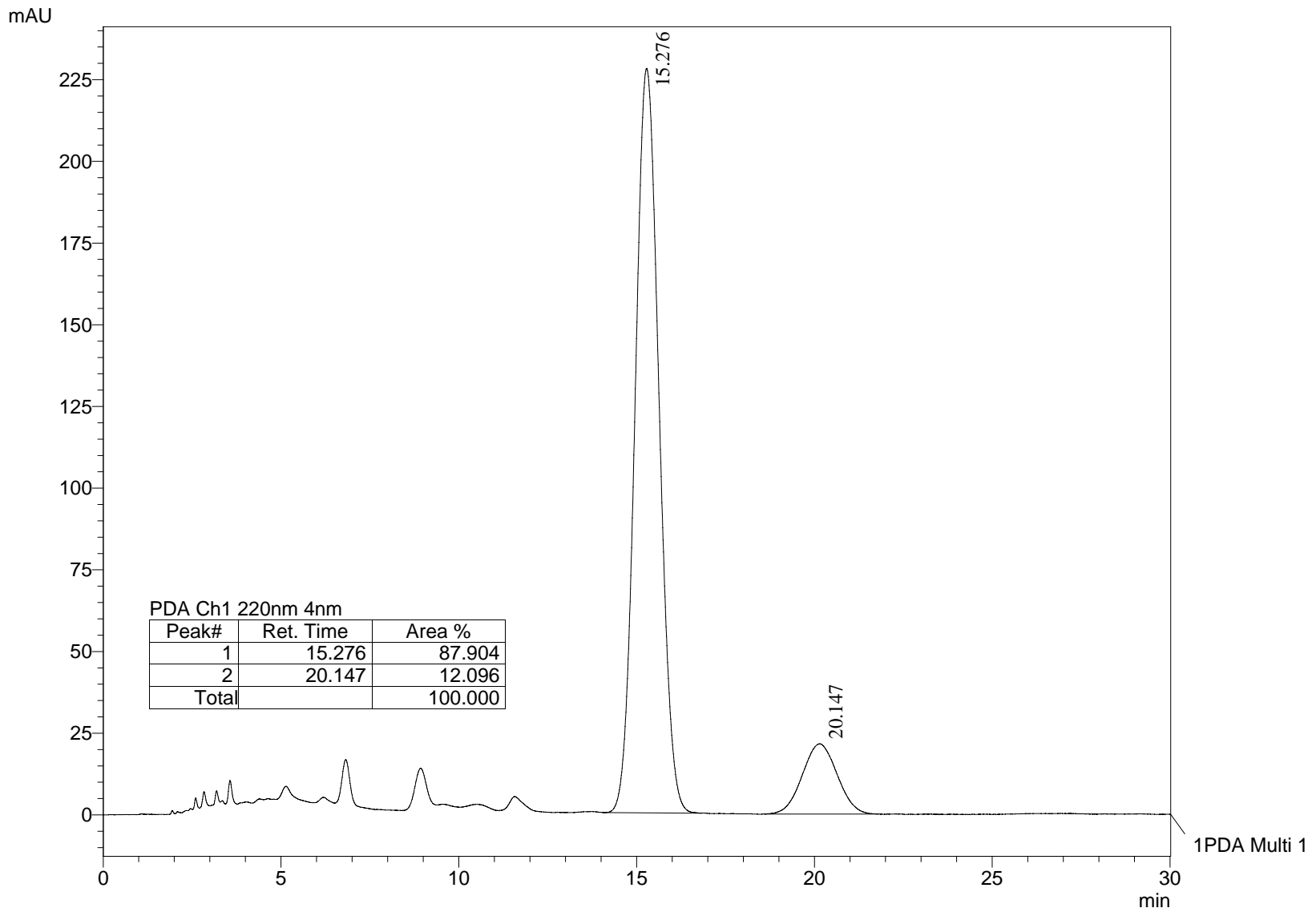




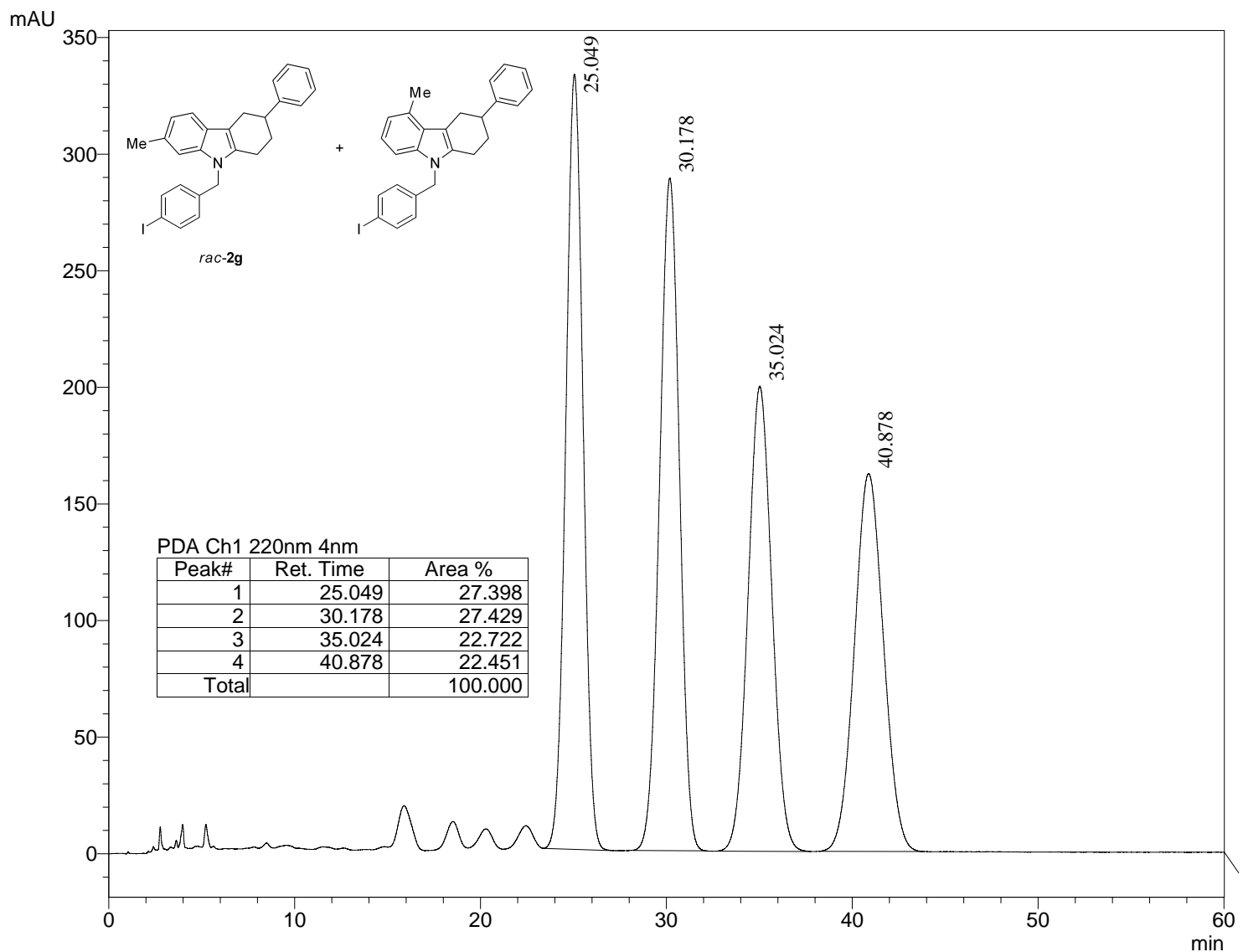

1PDA Multi 1

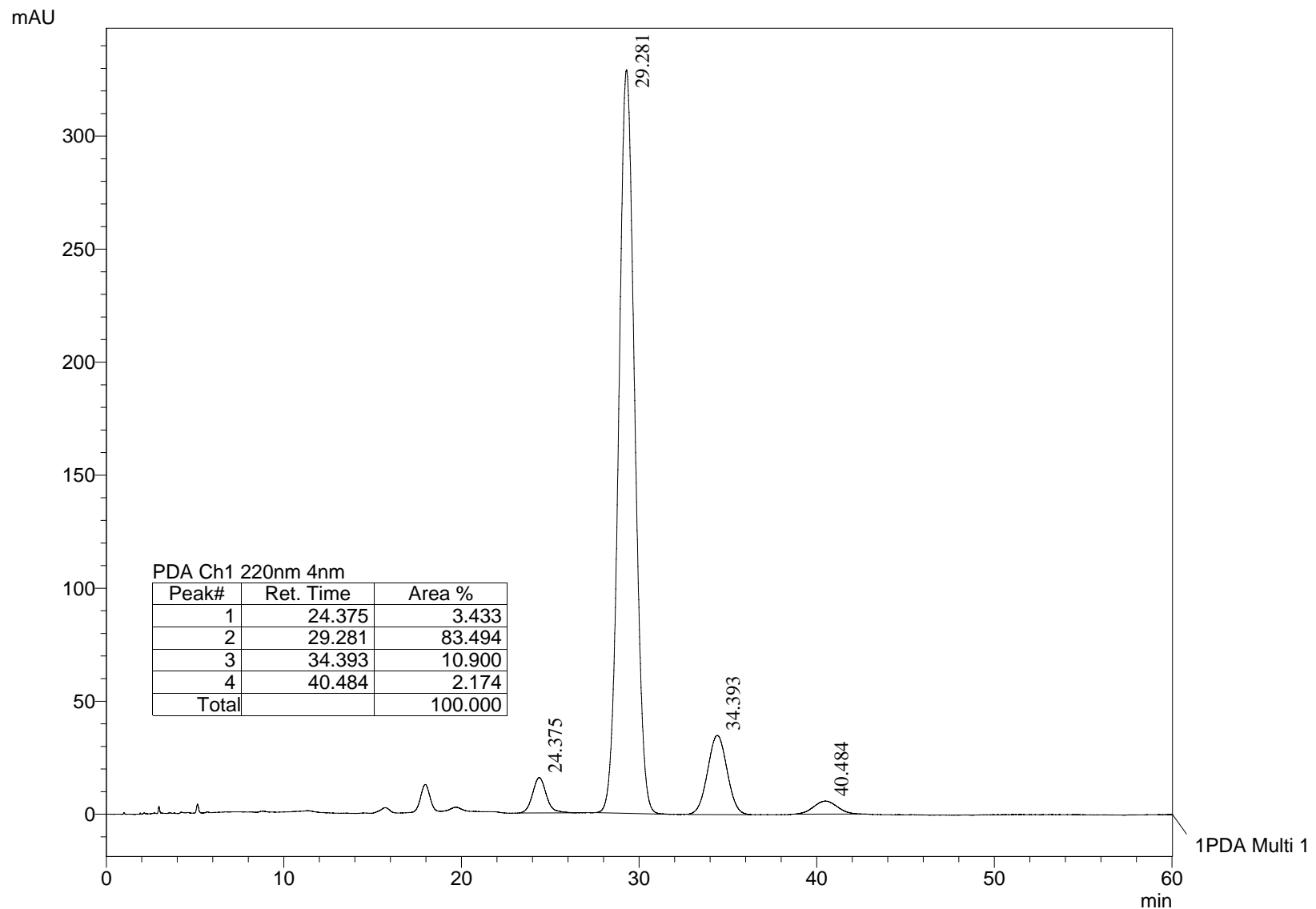



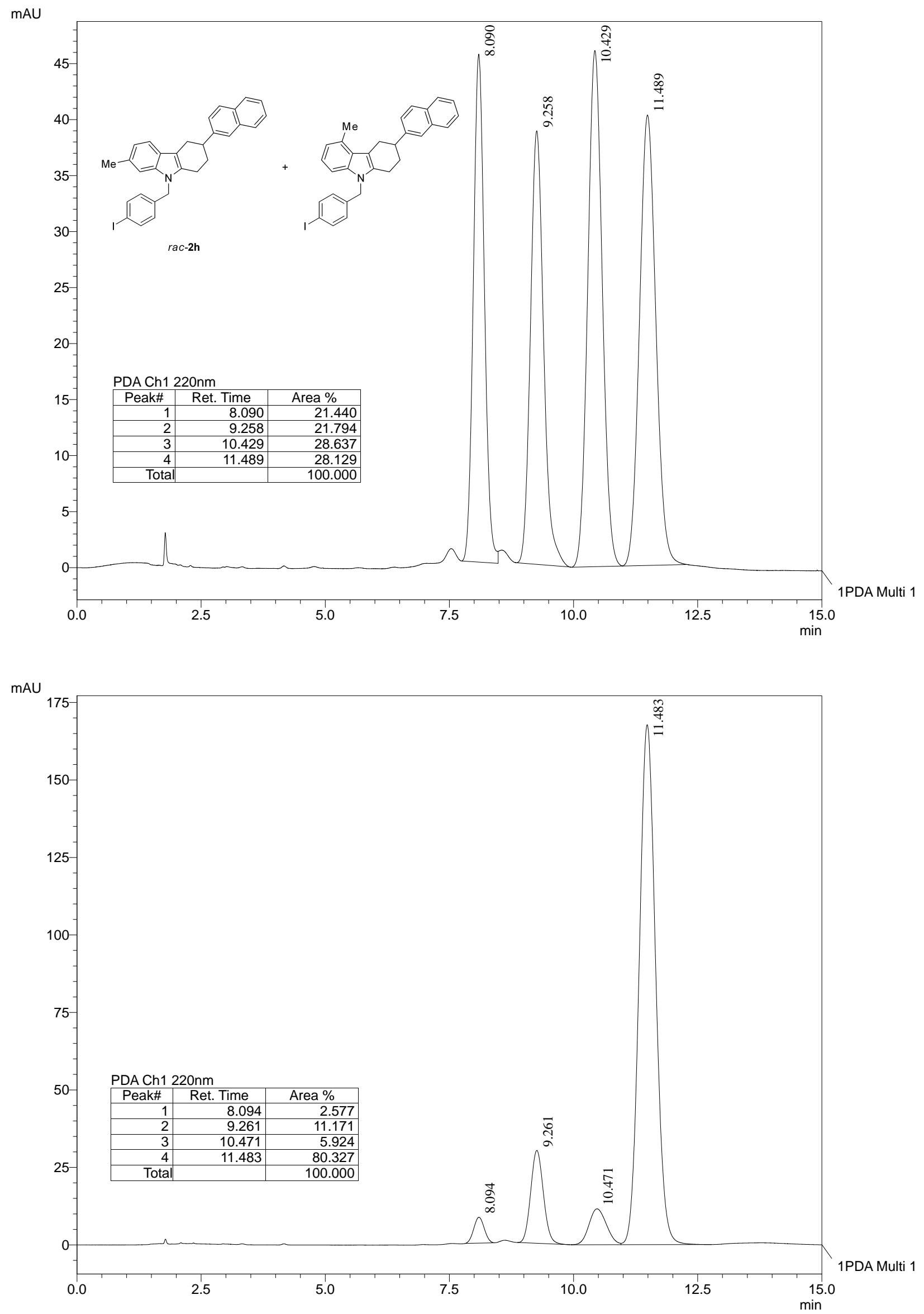


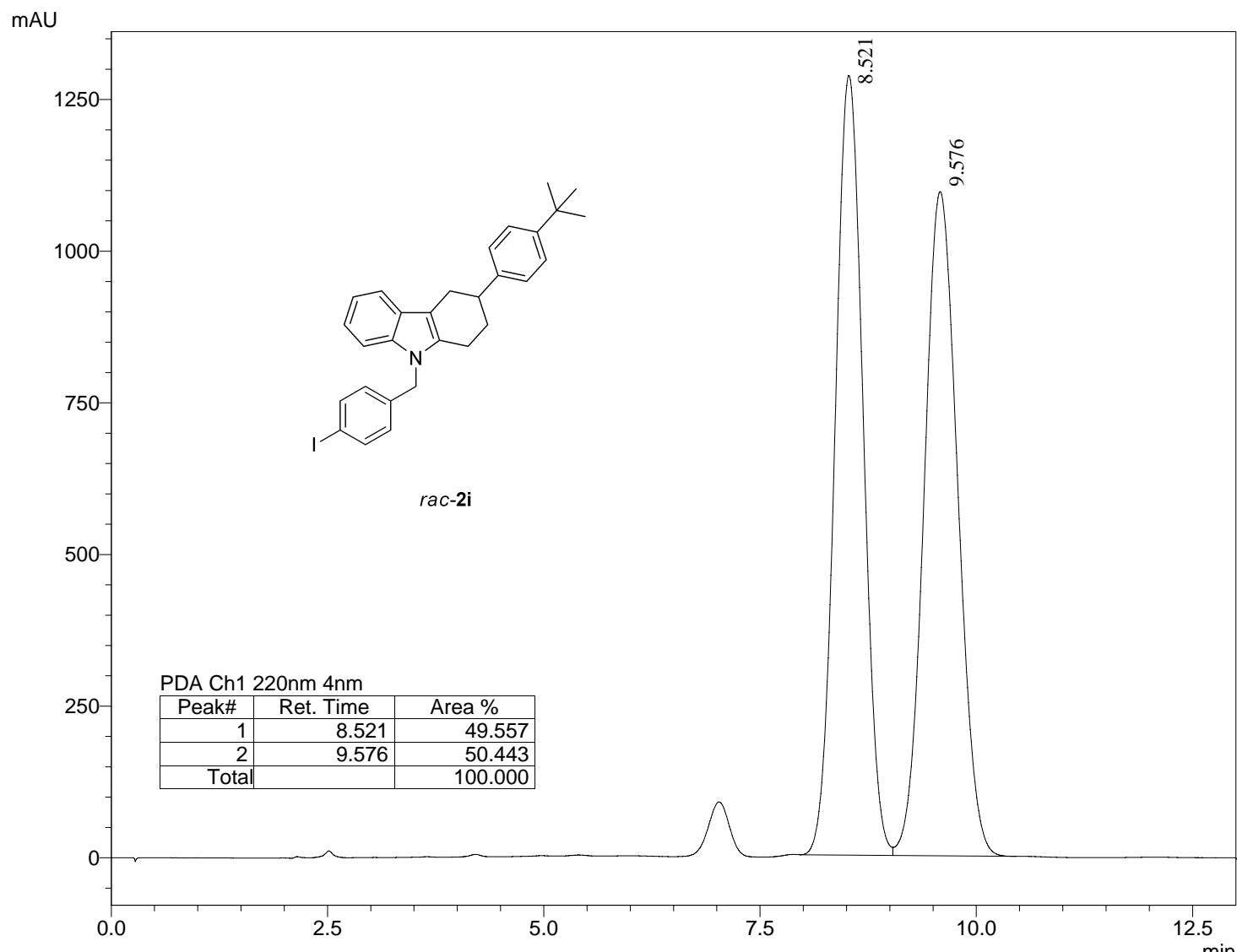

1PDA Multi 1

mAU

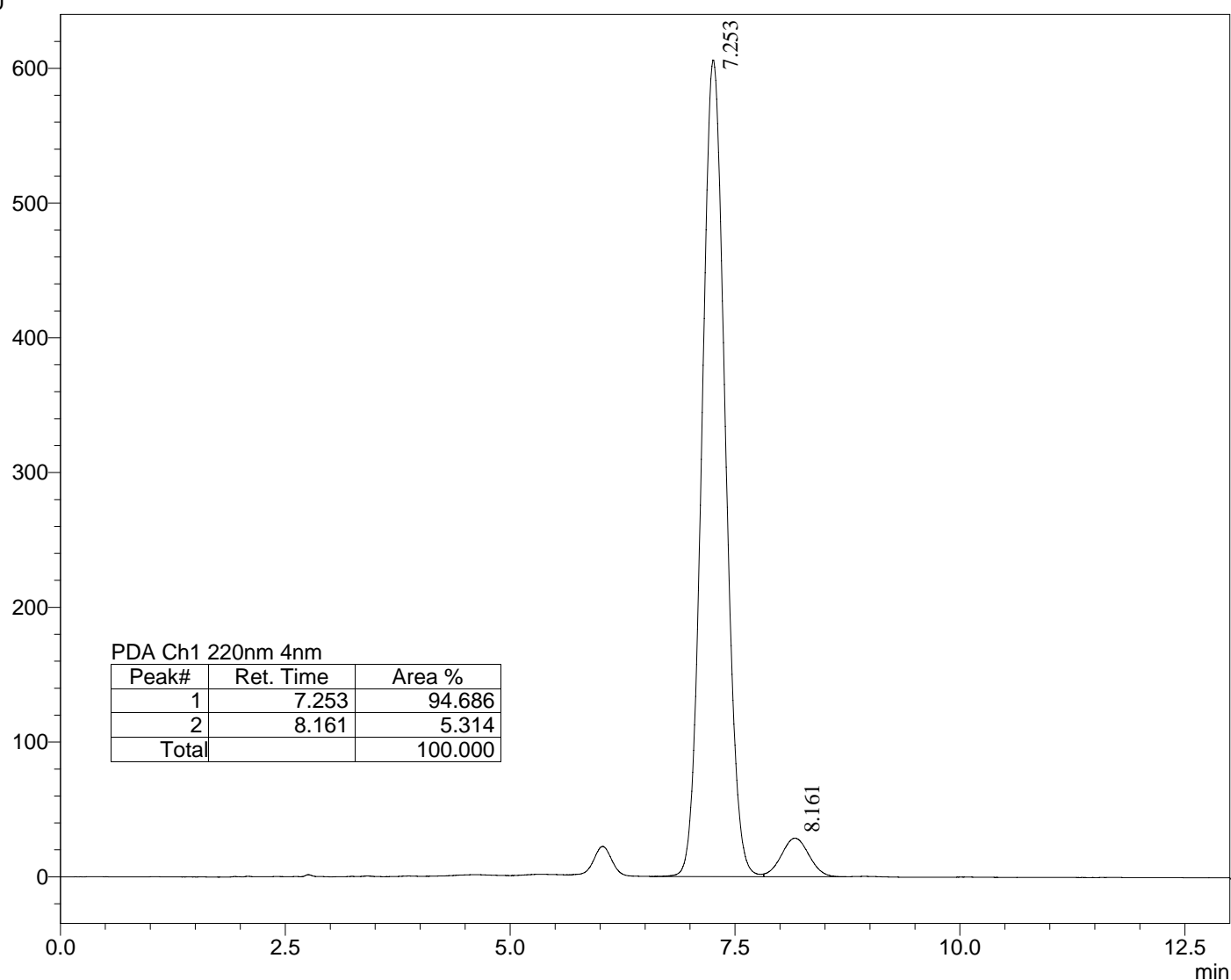


mAU

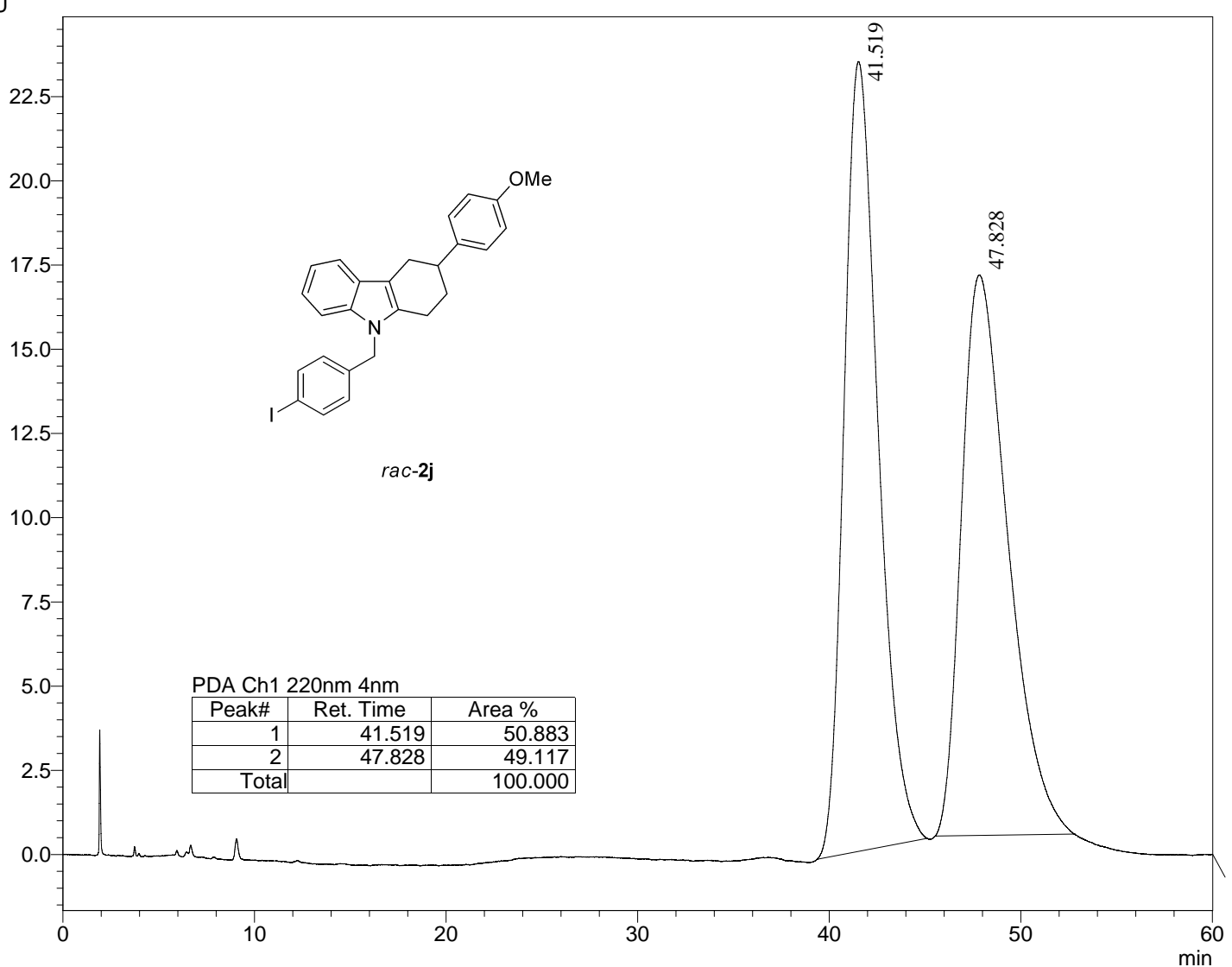

1PDA Multi 1

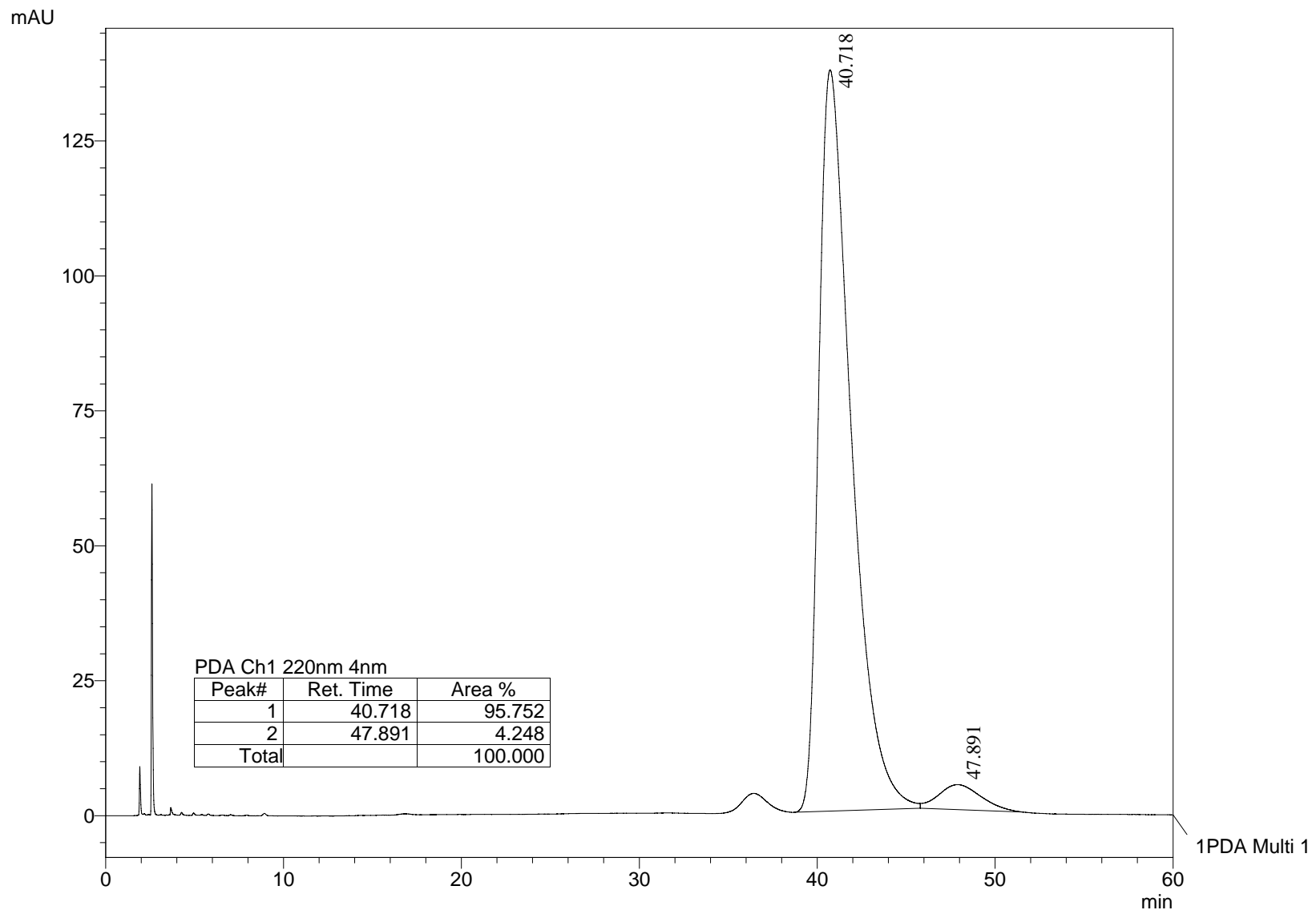


mAU

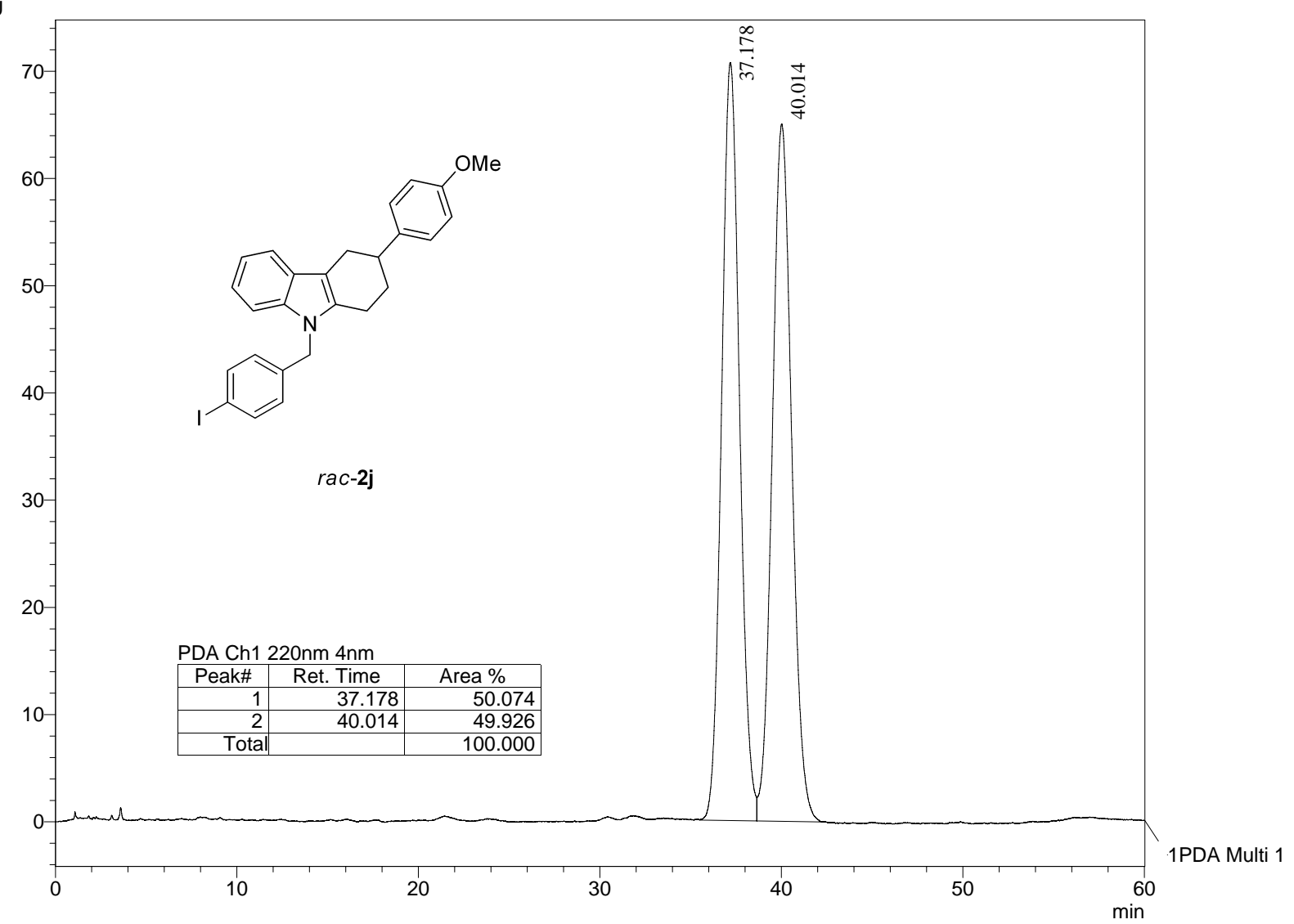

mAU

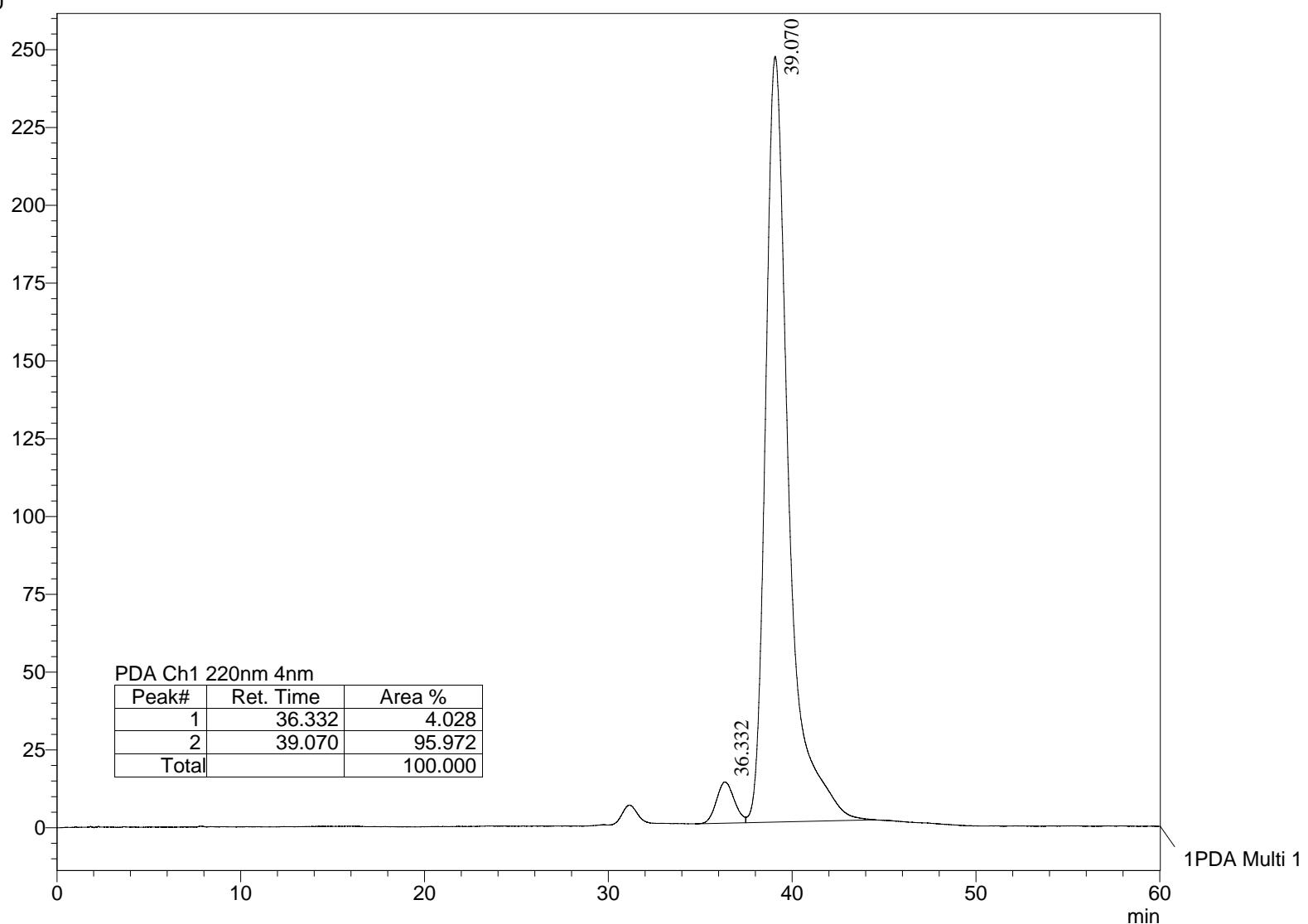



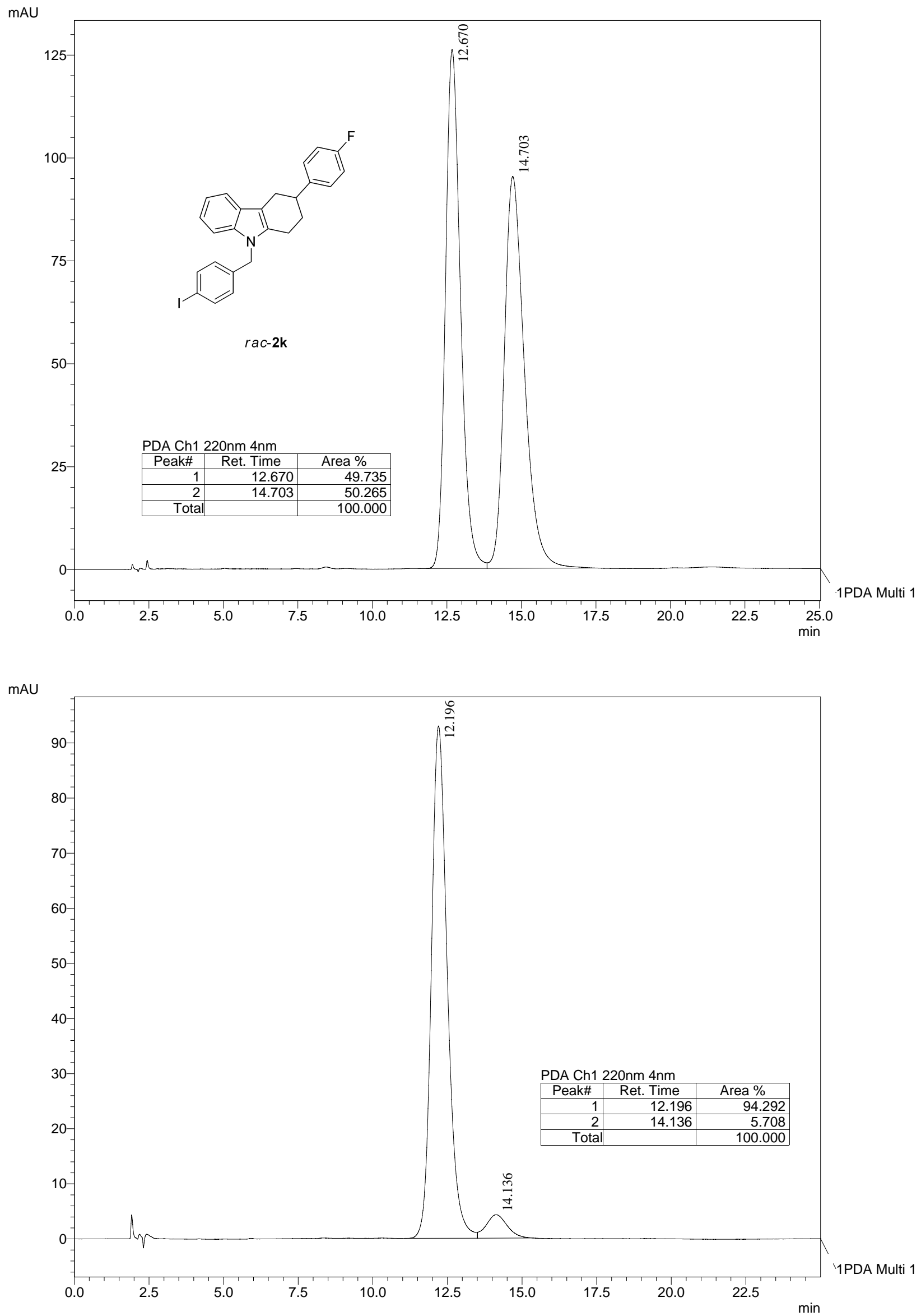
$\mathrm{mV}$

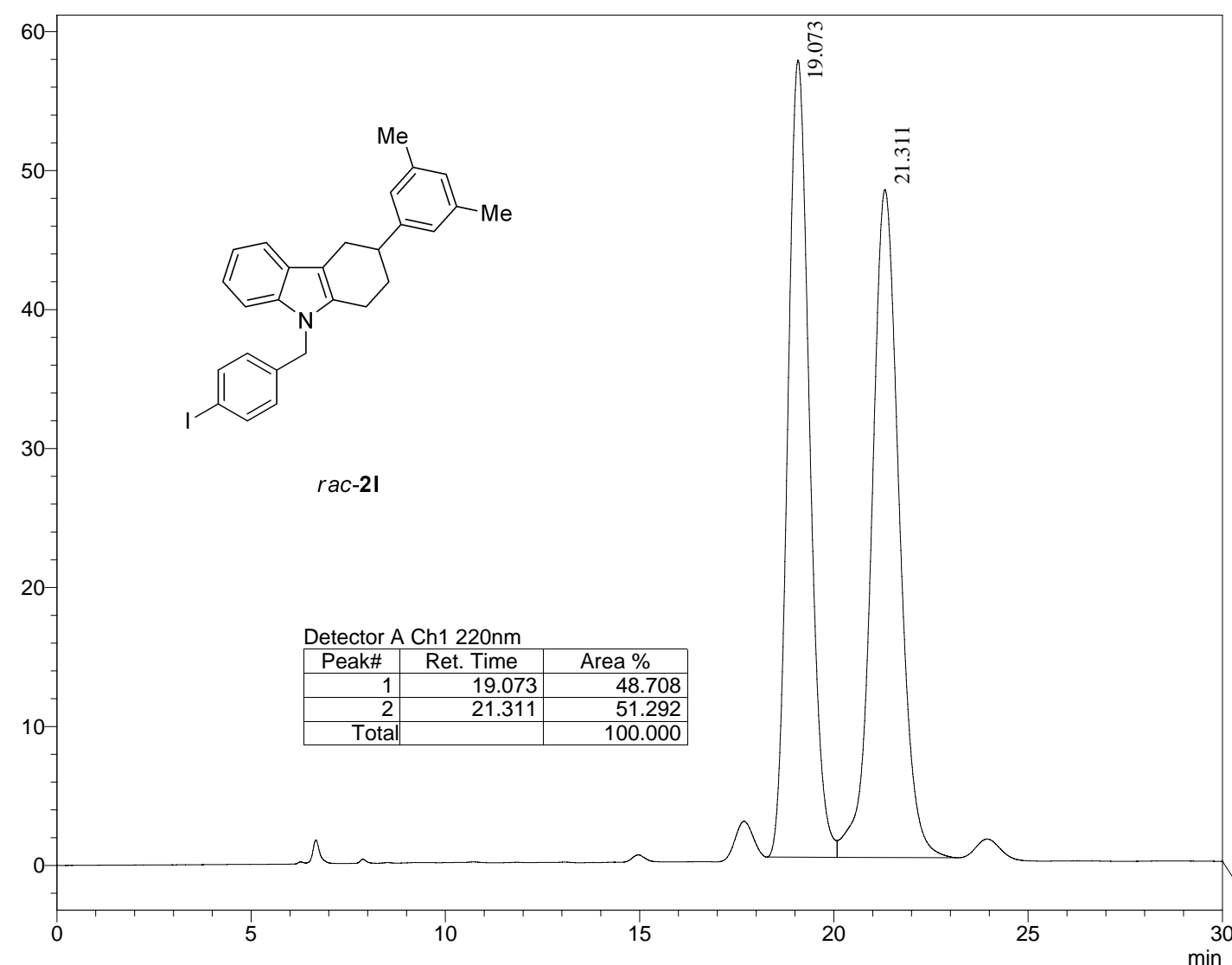

1Det.A Ch1

$\mathrm{mV}$

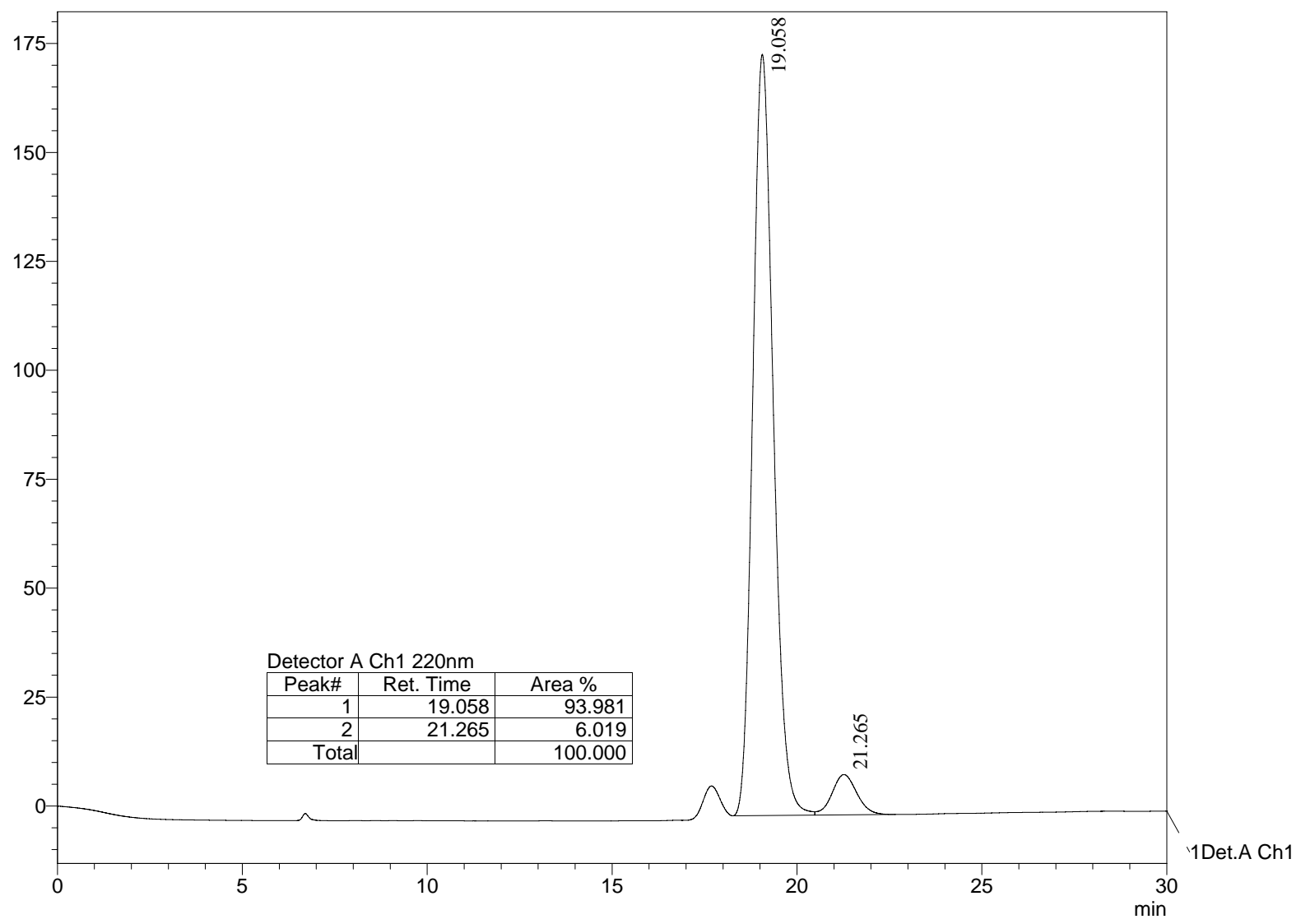




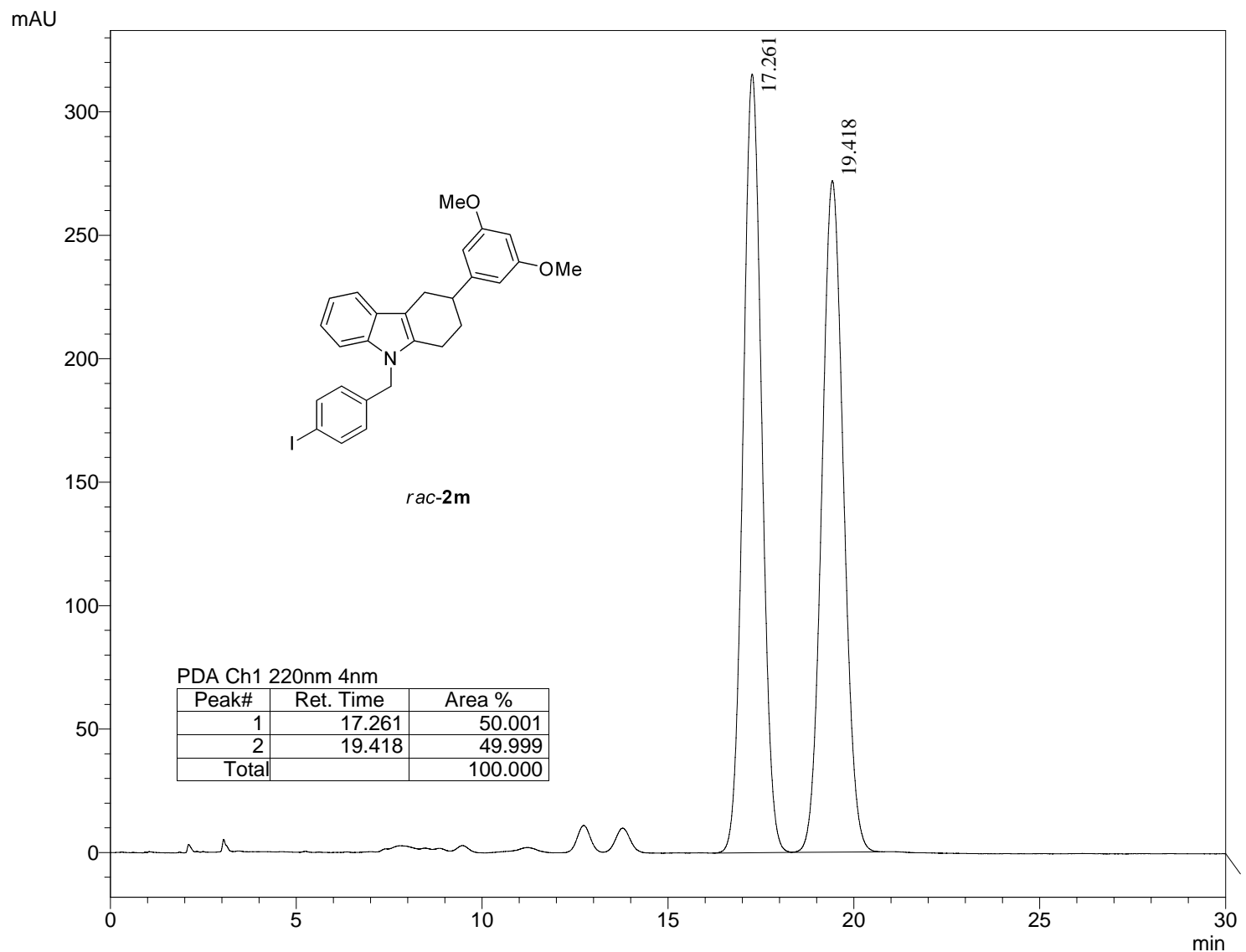

1PDA Multi 1

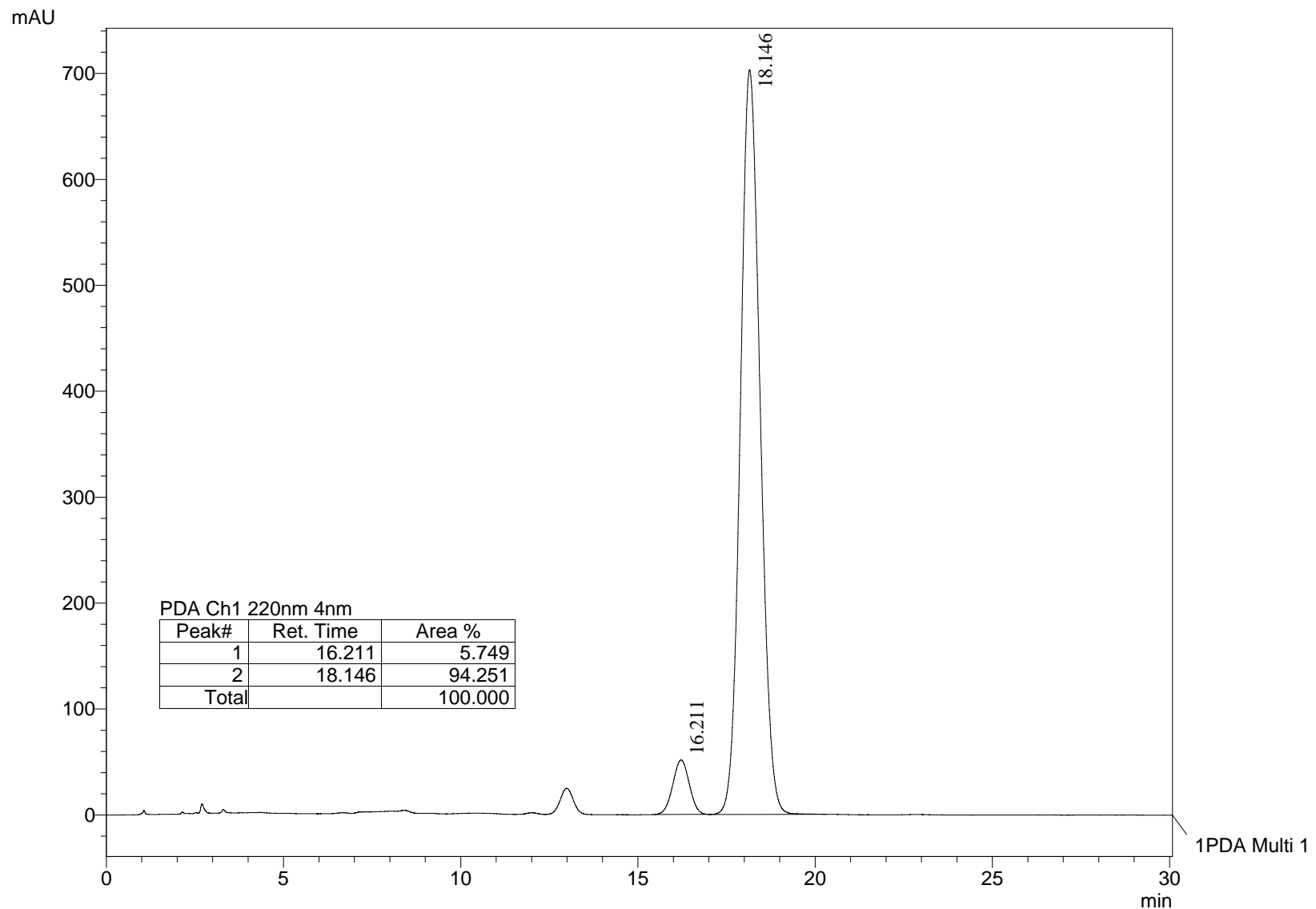




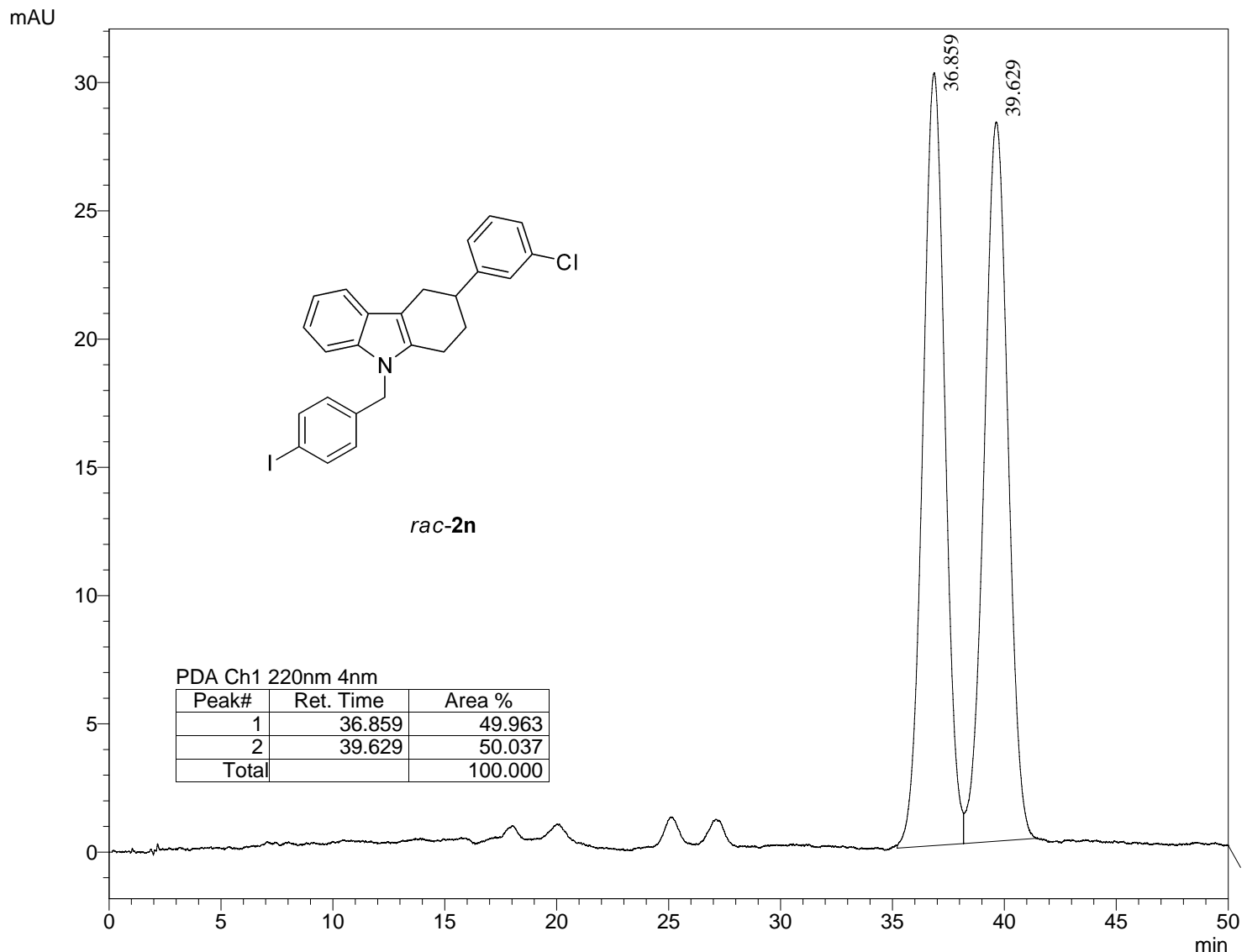

1PDA Multi 1

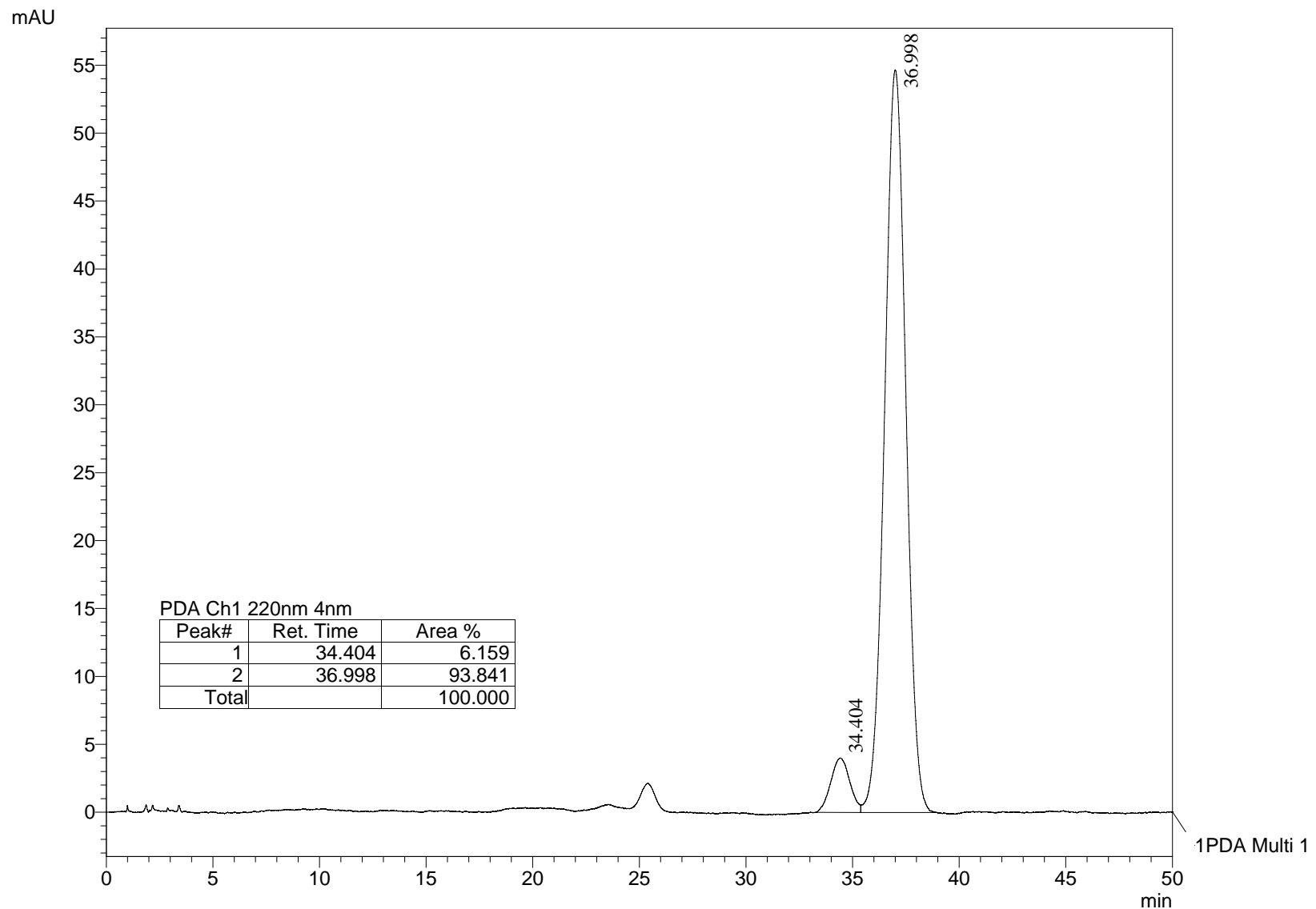




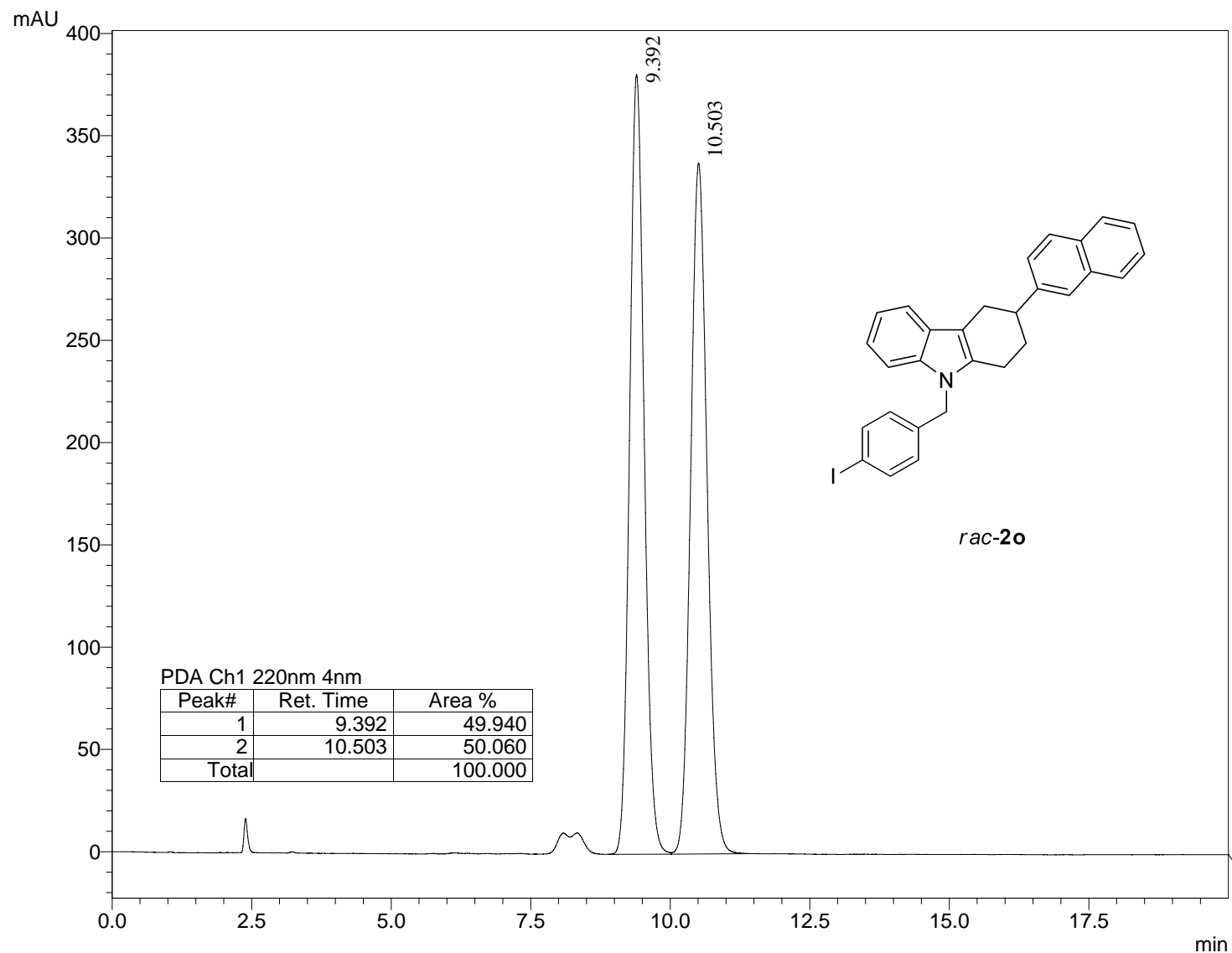

1PDA Multi 1

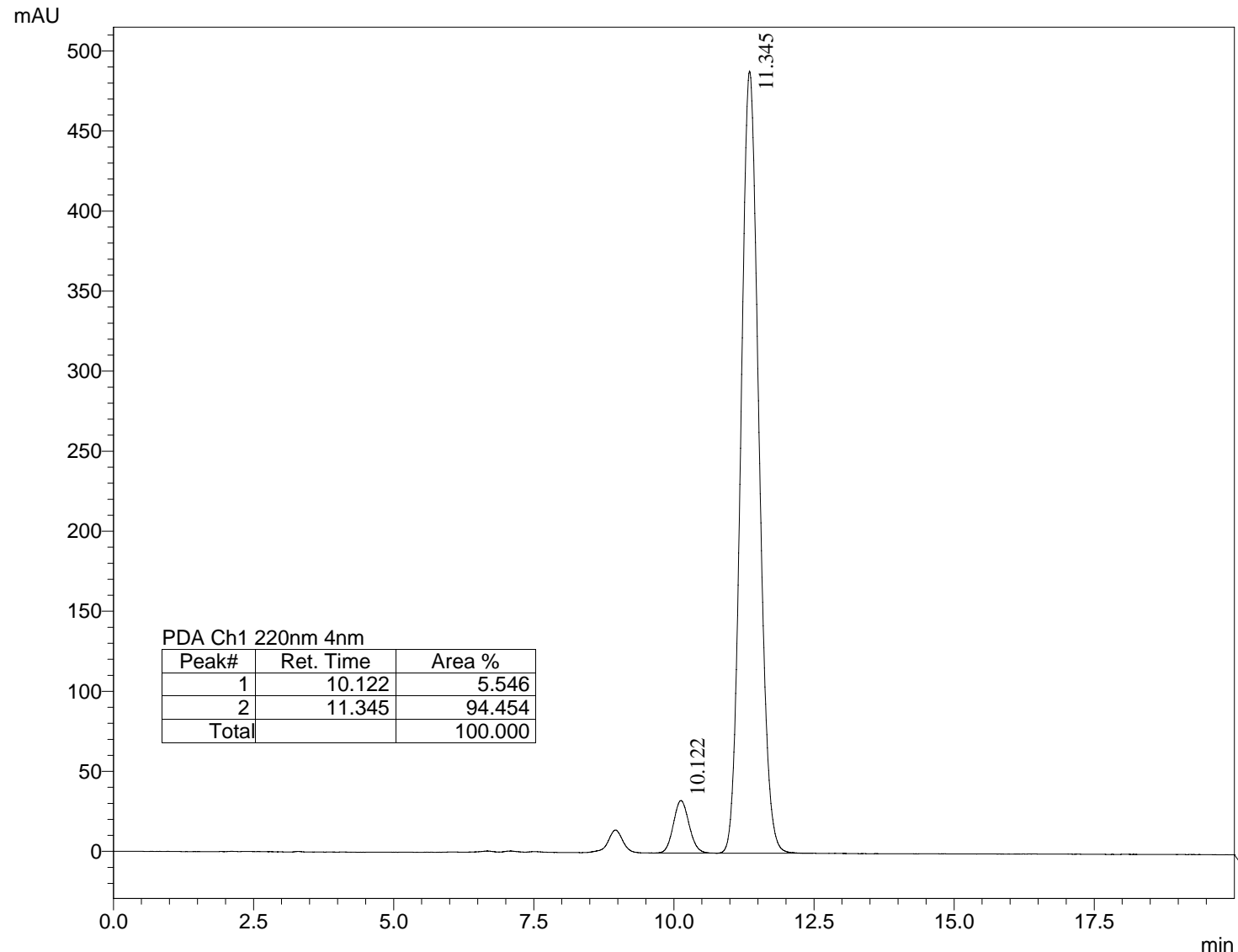




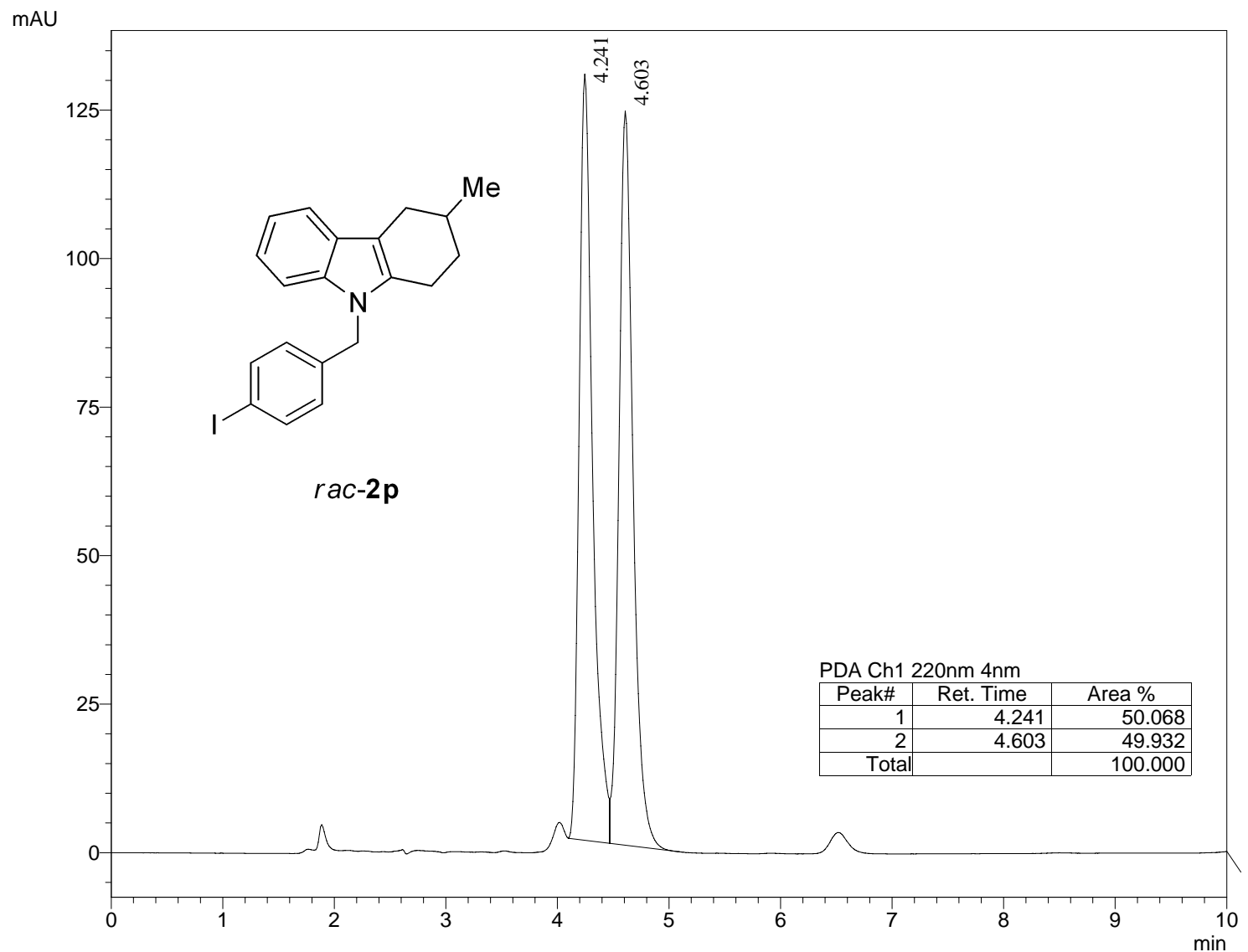

1PDA Multi 1

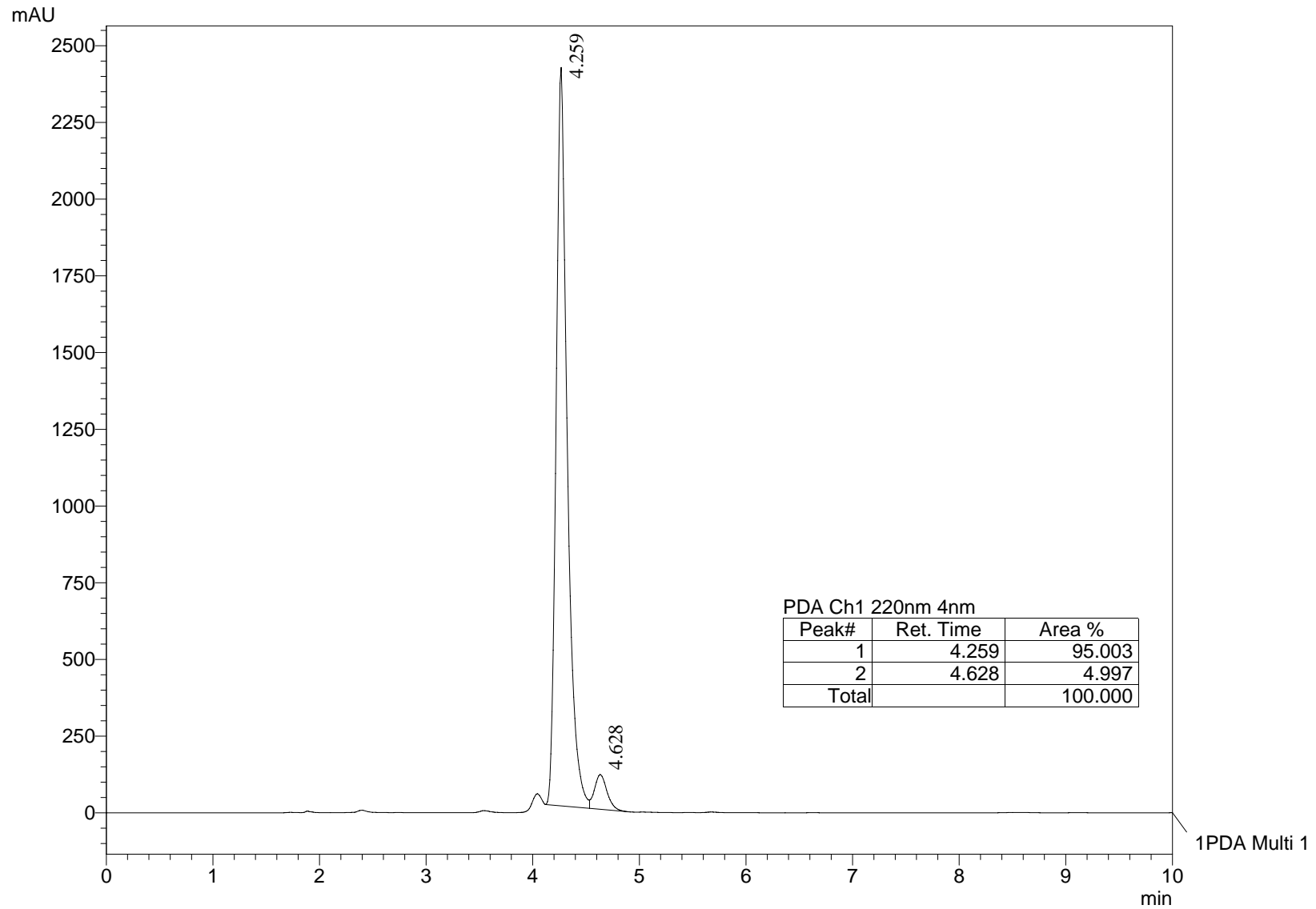



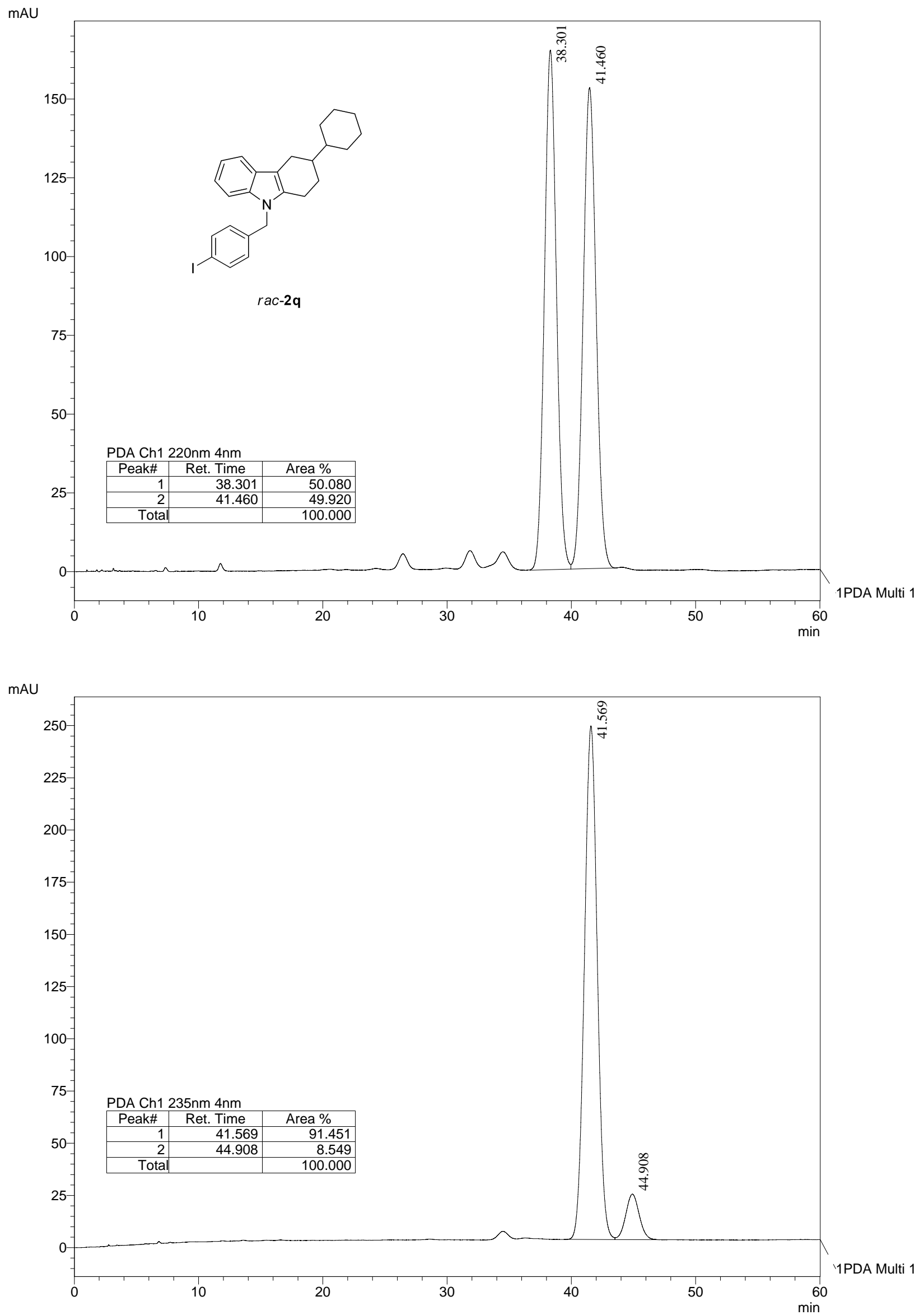

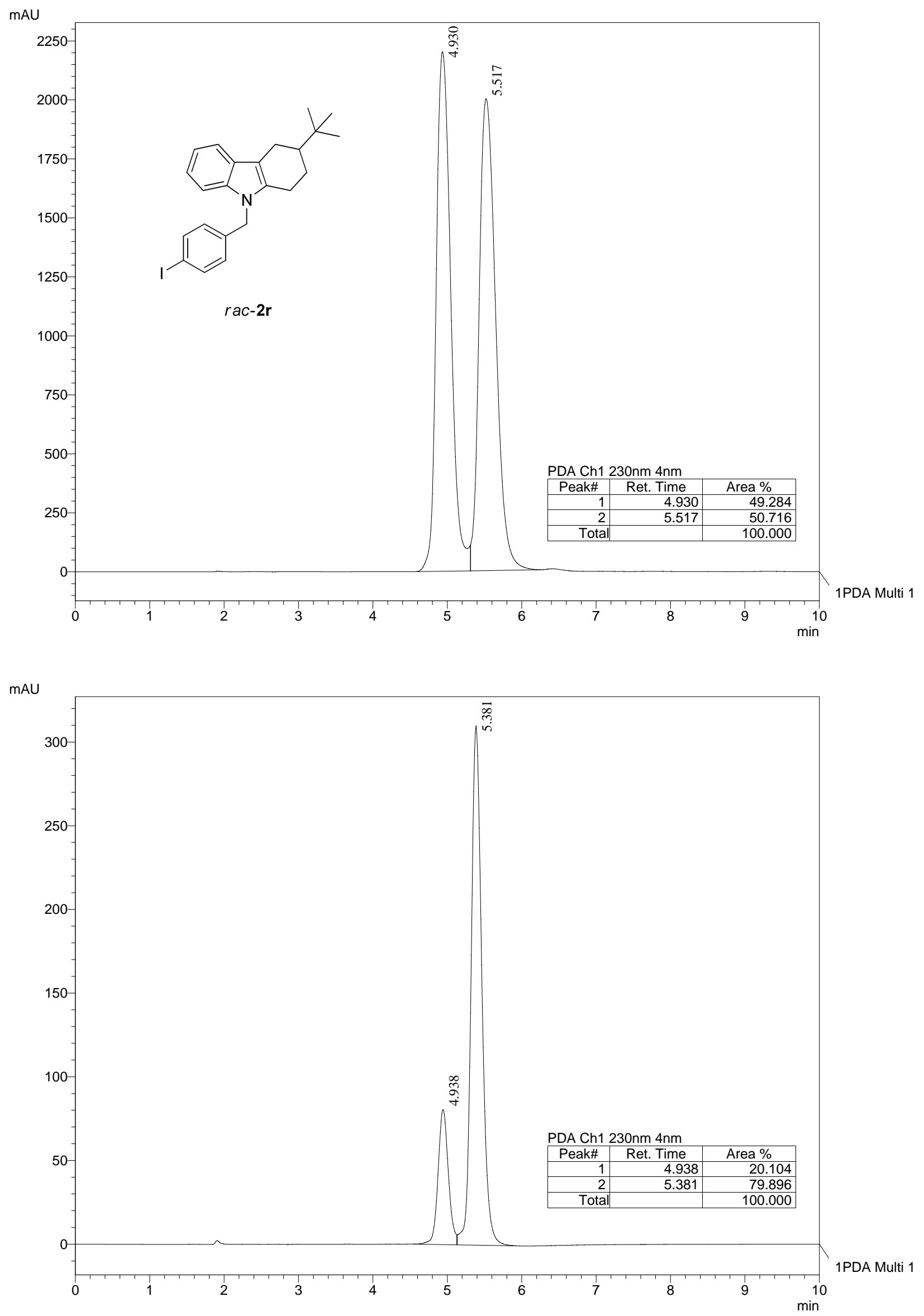


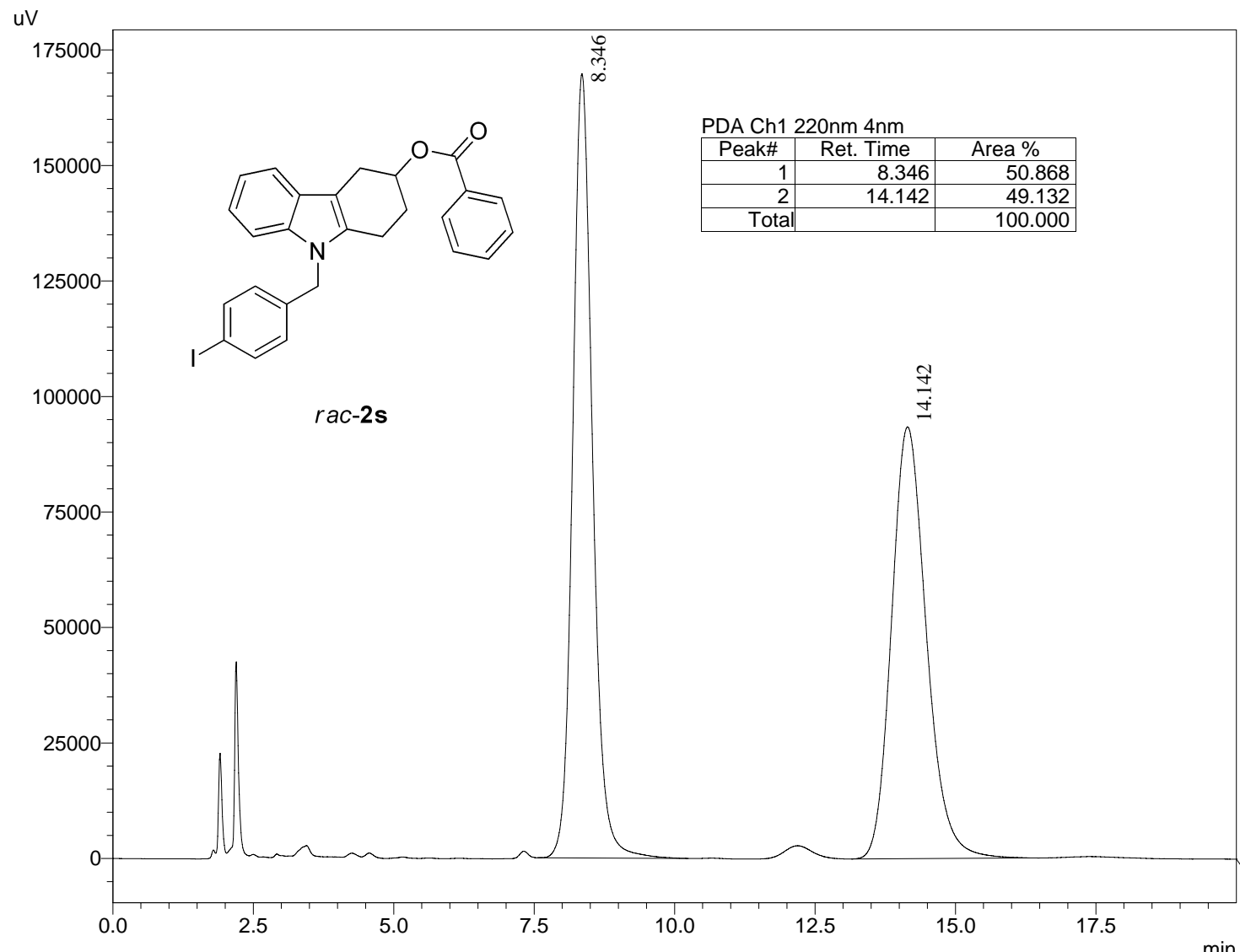

1PDA Multi 1

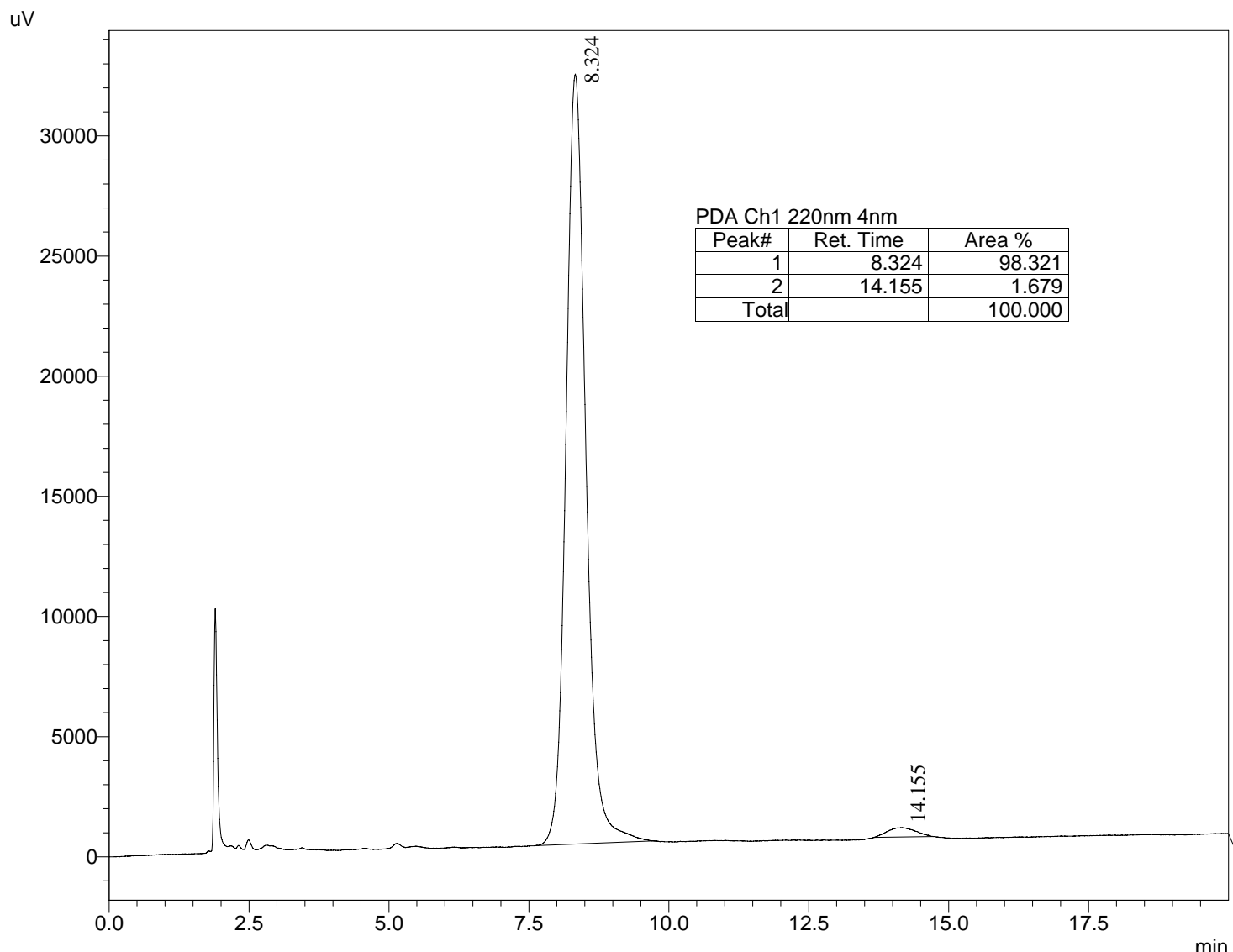




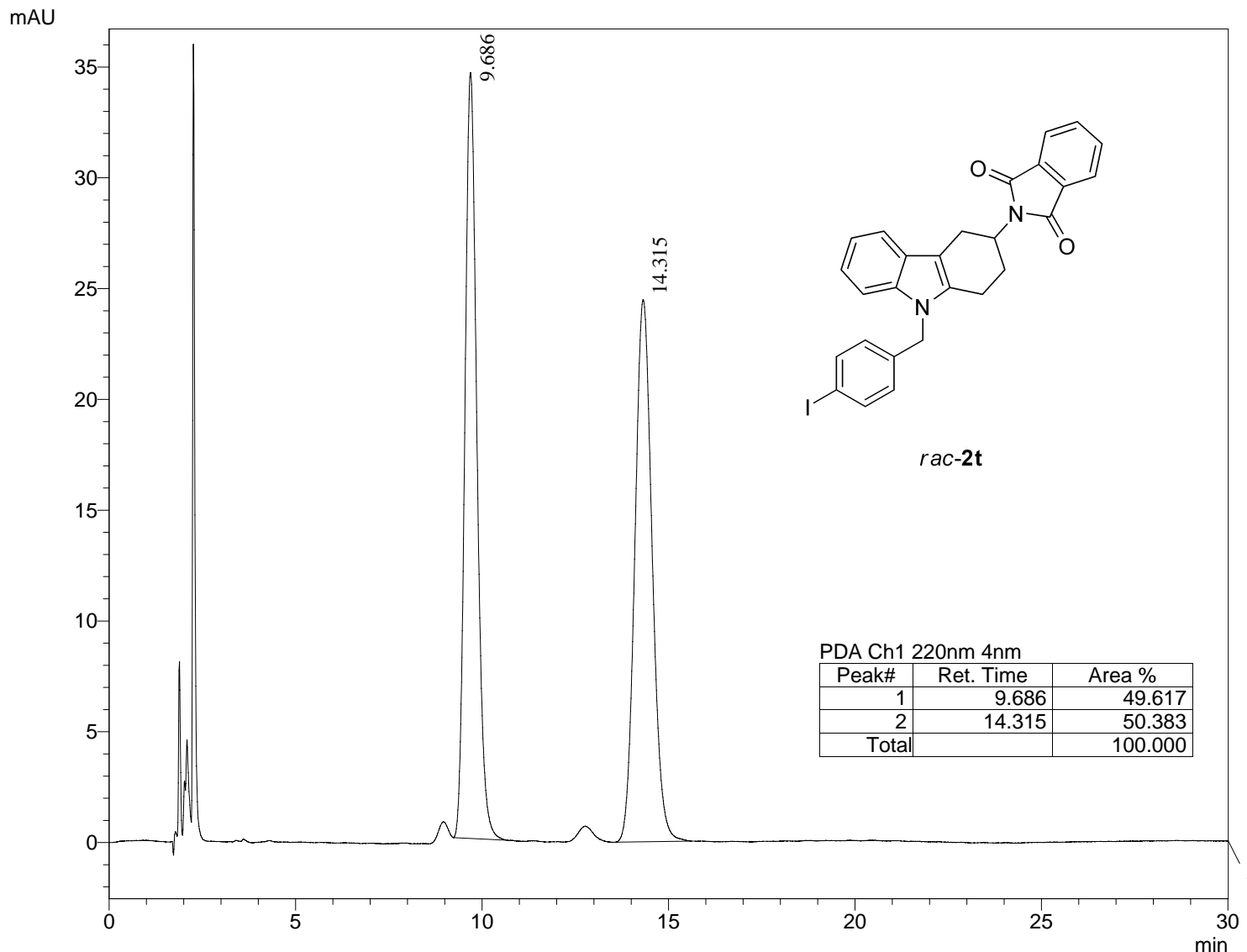

1PDA Multi 1

mAU

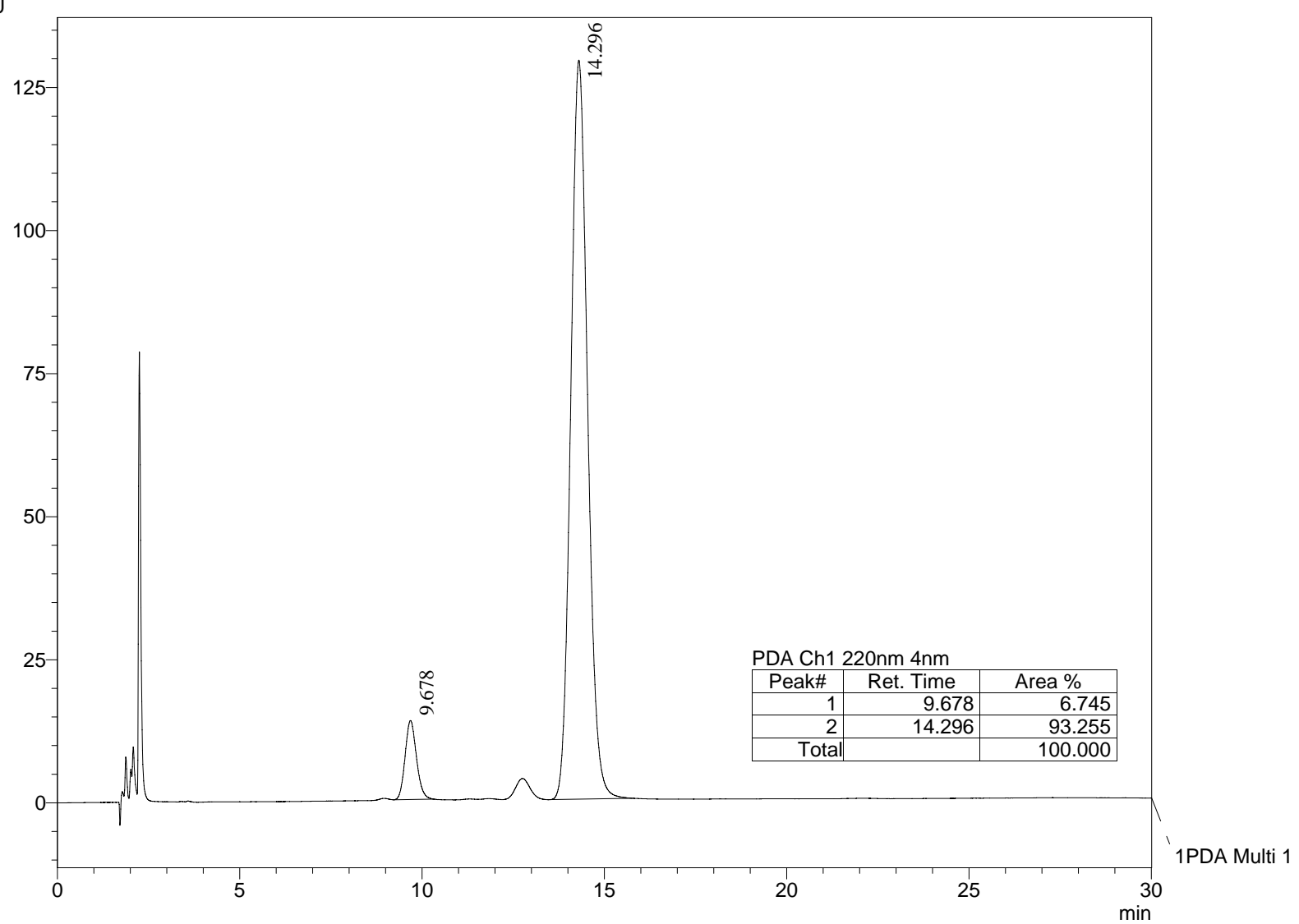




\section{Large scale synthesis of $\mathbf{2 t}$}

$\mathrm{uV}$
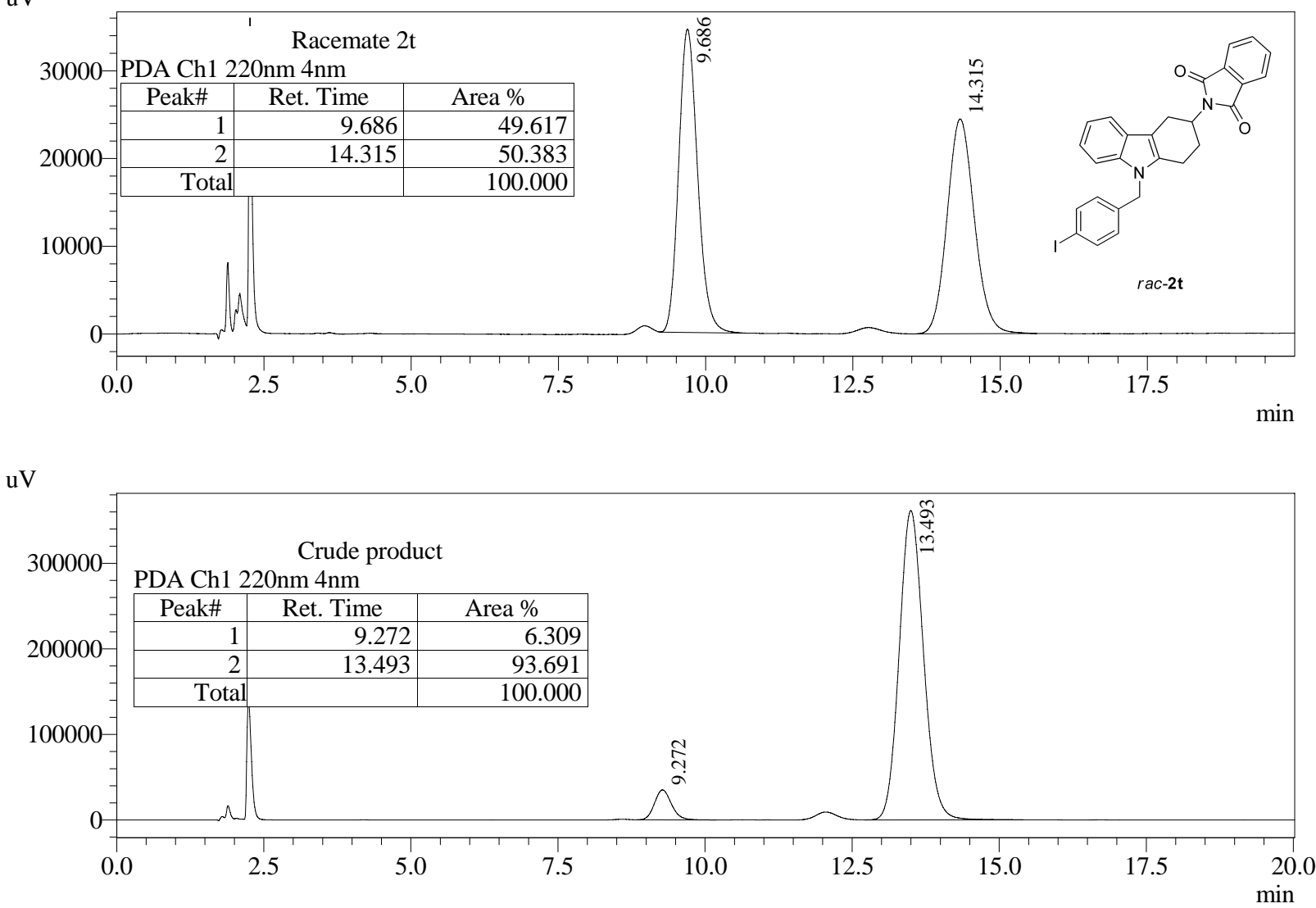

$\mathrm{uV}$

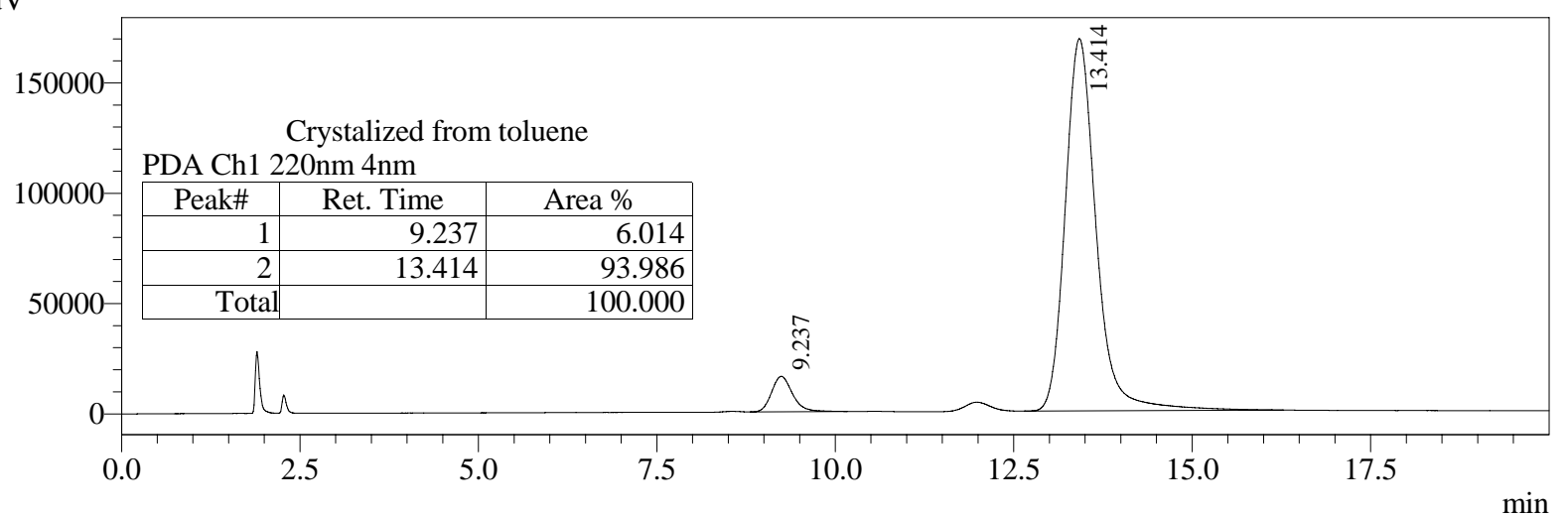

$\mathrm{uV}$

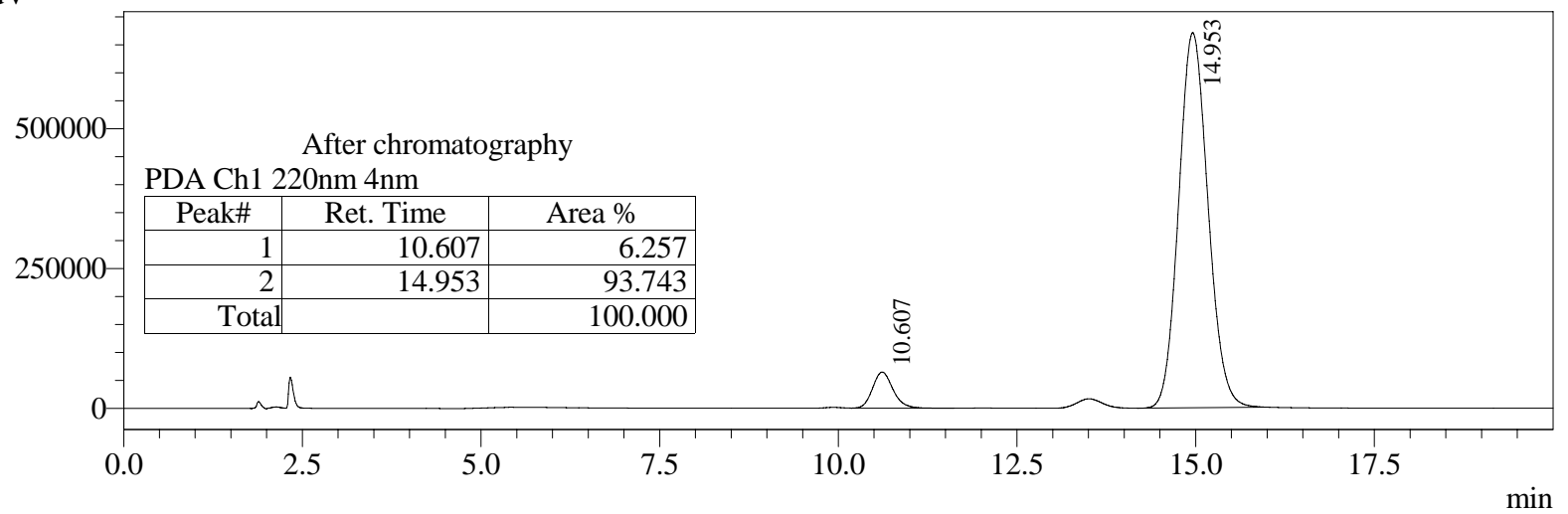




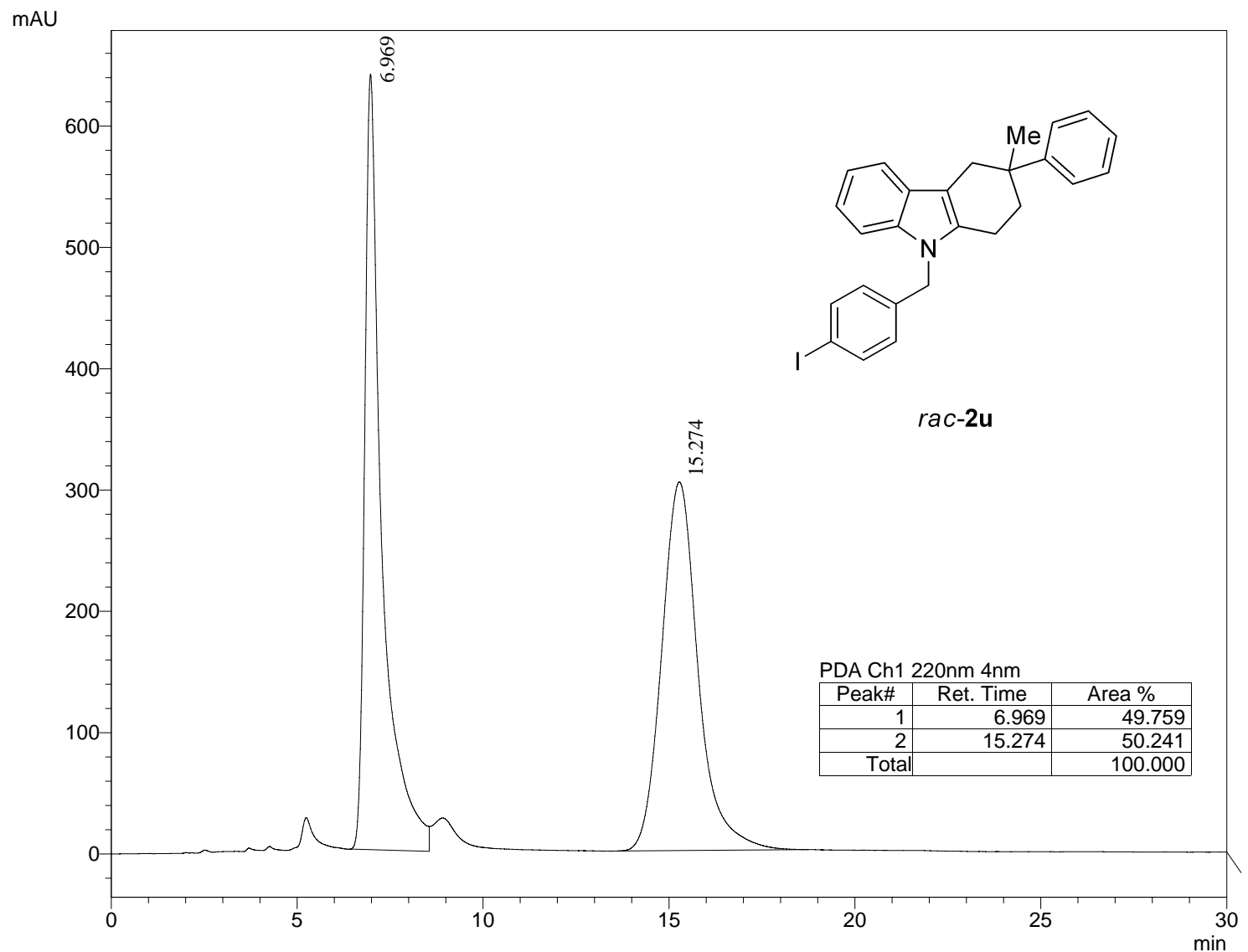

1PDA Multi 1

mAU

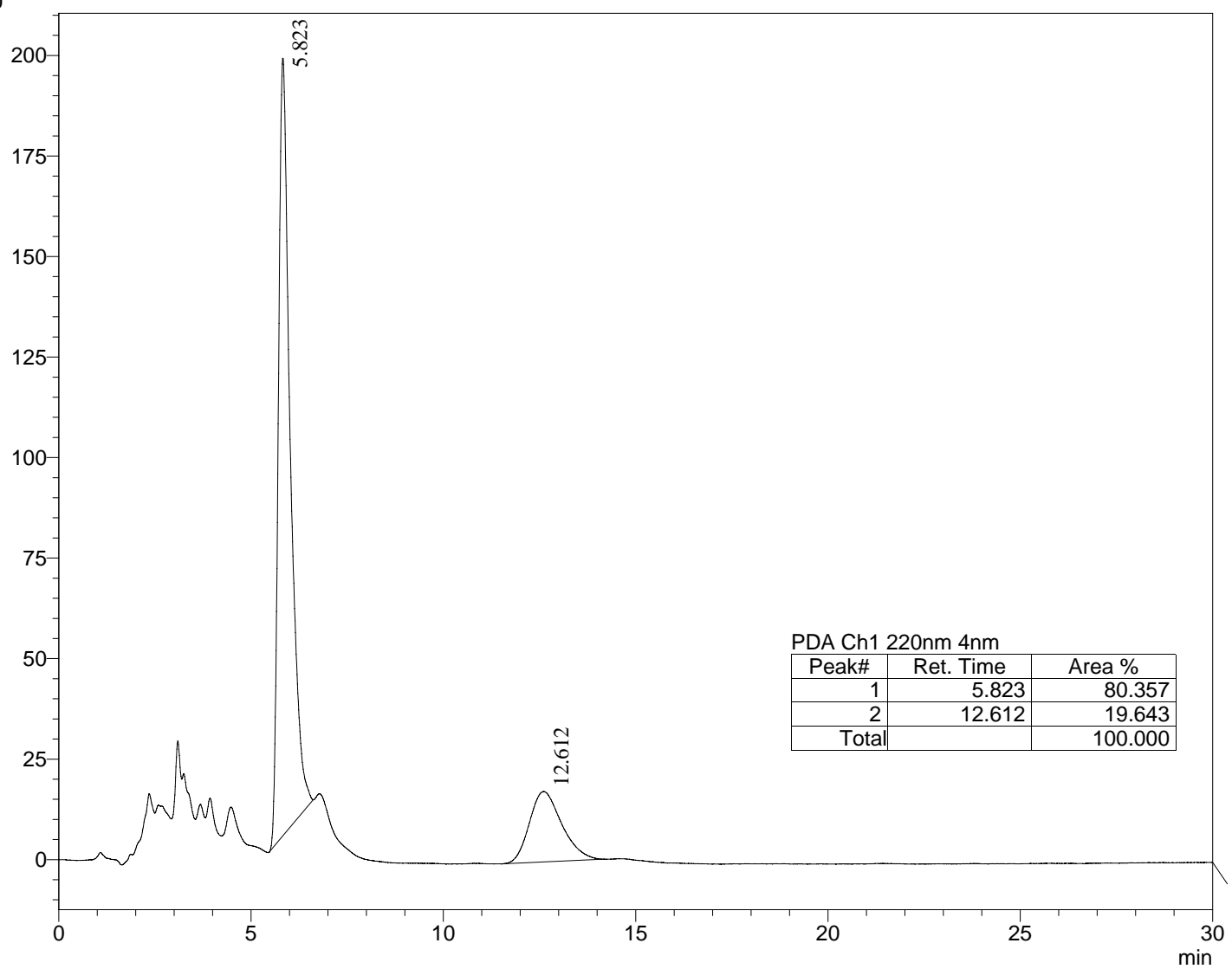

1PDA Multi 1 
mAU
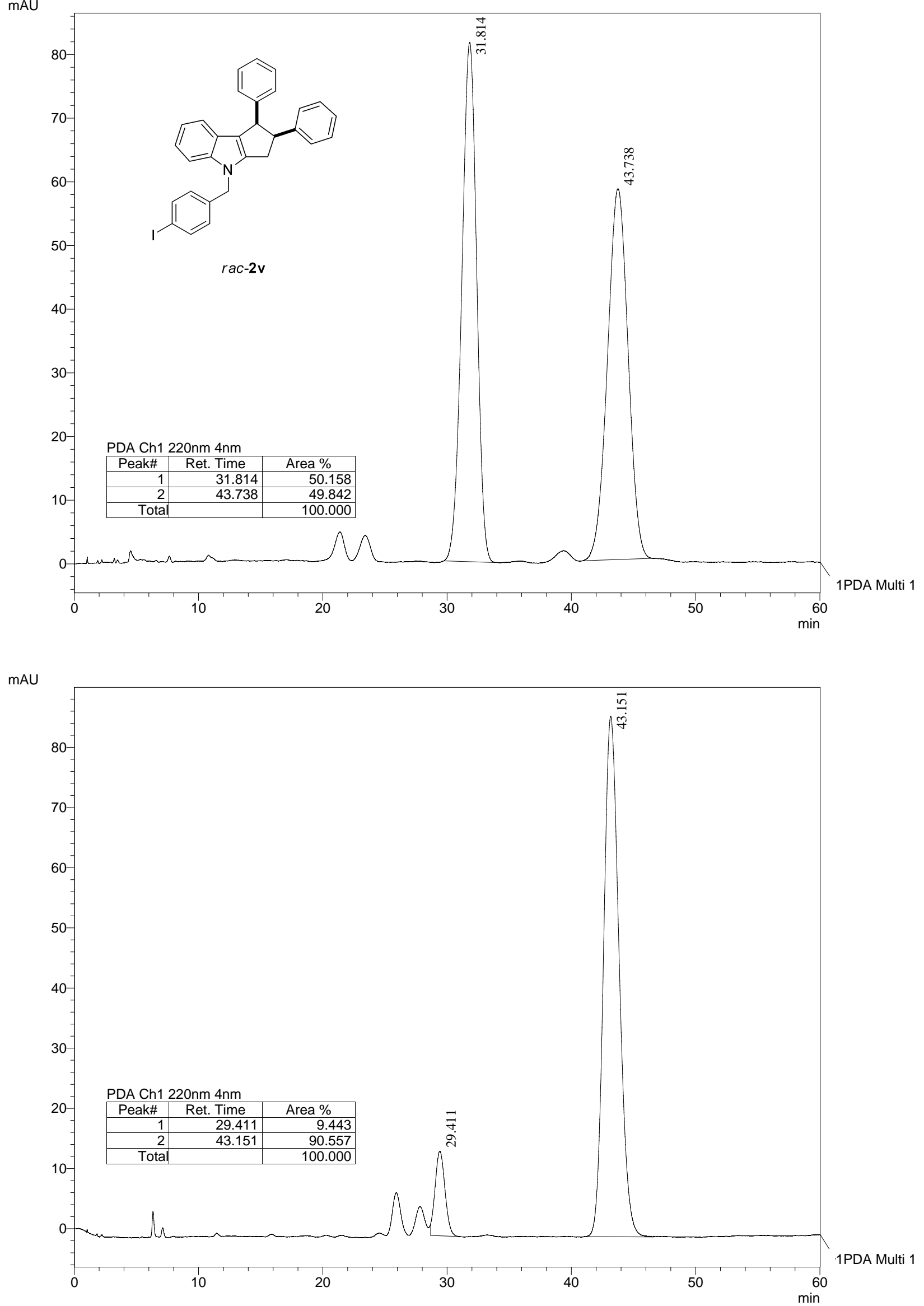

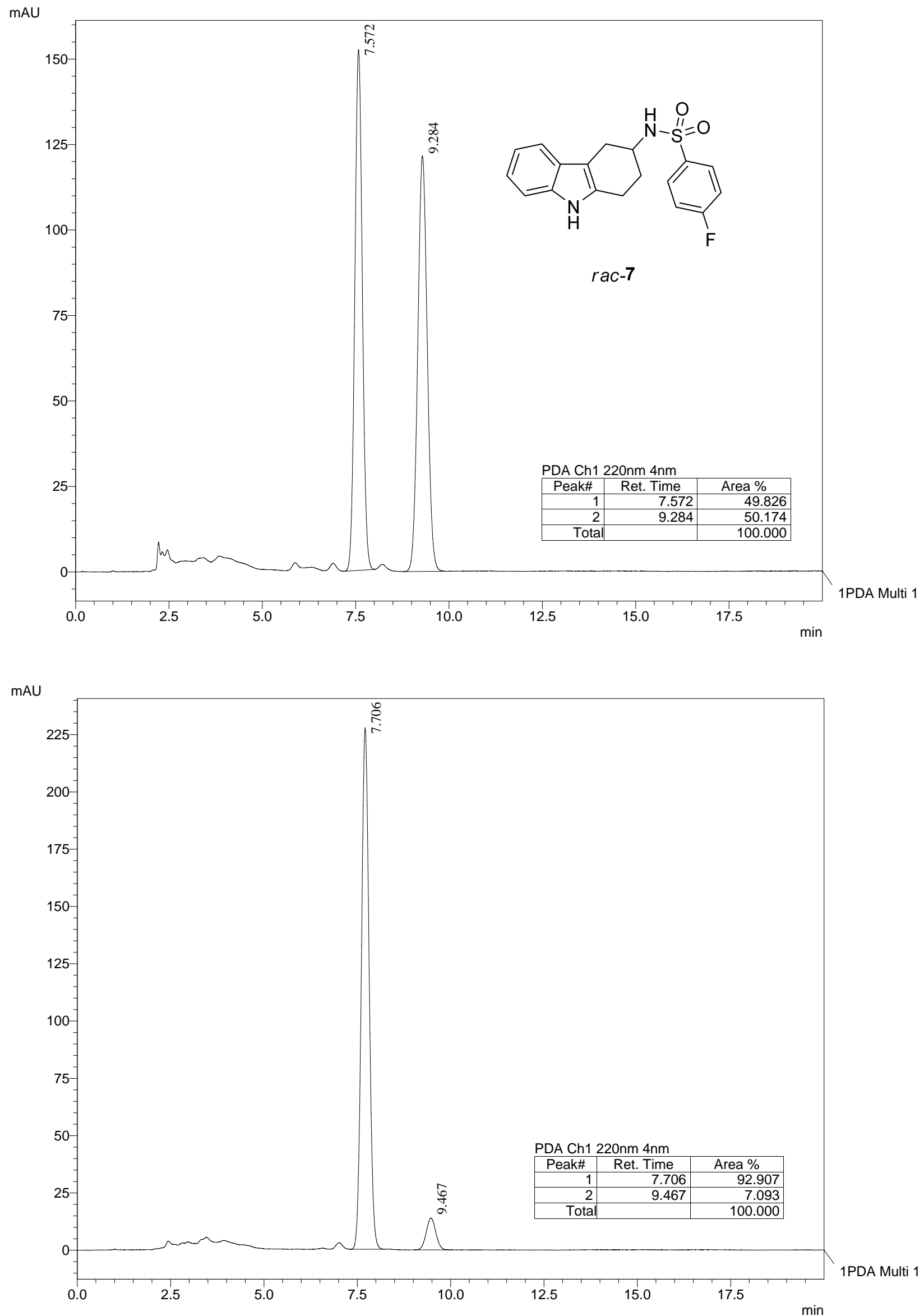